\title{
UPPER PALEOZOIC DEPOSITIONAL AND \\ DIAGENETIC FACIES IN A MATURE PETROLEUM PROVINCE \\ (A FIELD GUIDE TO THE GUADALUPE AND SACRAMENTO MOUNTAINS)
}

\author{
By \\ Peter A. Scholle and Robert B. Ha1ley \\ U.S. Geological Survey, Denver, Colorado 80225
}

Open-File Report $80-383$

1980 


\section{Contents}

Part I Permian reef complex, Guadalupe Mountains

Int roduction-- 1

E1 Paso-Carlsbad roadlog--_-_-_-_- 11

McKittrick Canyon roadlog-_- 32

Walnut Canyon roadlog-_- 37

Dark Canyon-Sitting Bull Falls-Rocky Arroyo roadlog-_-_-_-_-_-_ 42

I1lustrations-_-_-_-_- 55

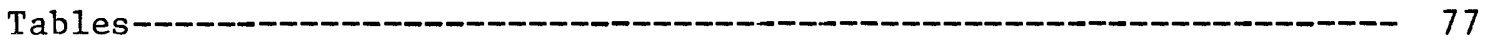

Bibliography-_-_- 117

Part II Upper Paleozoic bioherms, Sacramento Mountains

Int roduction-- 141

Carlsbad-Alamogordo roadlog-- 148

Alamogordo-E1 Paso roadlog- 155

I1lustrations-- 159

Tables-_- 162

Bibliography--_- 184 


\section{PART I}

THE PERMIAN REEF COMPLEX OF THE GUADALUPE MOUNTAINS

P. A. SCHOLLE 


\title{
Introduction
}

\author{
Setting
}

The Permian Basin region (fig. 1) provides an excellent opportunity to study the interrelationships of depositional facies, diagenetic alteration patterns, oil generation and migration, and ultimately, petroleum potential and production. The entire depositional spectrum from far-back-reef to deep basin can be observed in the Guadalupe and Delaware Mountains with little or no structural deformation and very slight vegetation or soil cover. The reef complex of this region is also dissected by a series of deep canyons cut at right angles to the regional facies strike. These canyons provide crosssectional views of the lateral and vertical relations of environments through time. Finally, the region is rather exceptional in that, at the end of Guadalupian time, the entire suite of facies was essentially preserved (pickled) by extremely rapid deposition of evaporites (anhydrite, halite, sylvite, and more exotic salts). These evaporites filled the Delaware Basin and even covered adjacent shelf areas. Thus, original facies relations were preserved from extensive erosional modification, and late Tertiary uplift, coupled with dissolution of the very soluble evaporites, has led to resurrection of original (Permian) topography (Plate 1 , in pocket), greatly facilitating facies reconstruction.

In addition to the advantages provided by these outcrops, the Permian Basin has a wealth of subsurface data. More than 30,000 exploration wells and 150,000 development wells have been drilled in the Permian Basin region. All the outcrop facies of the Guadalupe, Delaware, and Glass Mountains are encountered in the subsurface Delaware Basin, Northwest Shelf, and Central Basin Platform as well as in the Midland Basin, and to a lesser degree, the Marfa Basin (fig. 1). Thus, the associations of oil and gas with specific depositional and diagenetic facies can be rather clearly established in this region.

\section{Previous studies}

A number of classic studies have been completed on the "Permian reef complex" of Texas and New Mexico which have established an excellent stratigraphic and sedimentologic framework for the region. Three early studies (King, 1948; Adams and Frenze1, 1950; Newe11, and others, 1953), in particular, presented the overall outlines of our modern concept of reefrelated depositional environments. Subsequent studies, including those of Babcock (1977), Dunham (1972), Esteban and Pray (1977), Harms (1974), Hayes (1964), Mazzullo and Cys (1977), Meissner (1969), Schmidt (1977), Tyrell (1969) and others, have fleshed in the details of many of the depositional environments and have contributed to our understanding of the diagenetic history of the region. In spite of this, however, few areas have more unresolved geological controversies than the Permian reef complex. Not a single one of the facies represented in the spectrum of basinal to far-backreef settings has not evoked a variety of opinions as to its origin or significance. Thus, although the overall environmental framework of facies is generally agreed upon, much work remains to be done on specific interpretations. 


\section{Depositional and stratigraphic setting}

The Permian reef complex is characterized by three sections of time equivalent but lithologically very dissimilar rocks. The first facies consists of thick masses of finely laminated siltstones and sandstones with thinner, interbedded black-gray limestone bodies. The second facies contains massive, light-gray limestones overlying steeply bedded, partially dolomitized, blocky limestone rubble. The third zone contains tan, finegrained, medium-bedded dolomites with interbedded evaporites and red to brown sandstone and siltstone units.

As early as the late $1920^{\prime} \mathrm{s}$, it was recognized that this represented a basin-reef-back reef sequence of environments (Lloyd, 1929; Cranda11, 1929; Blanchard and Davis, 1929). These conclusions were drawn largely on lithologic criteria. Subsequent work (eg. King, 1948; Newell and others, 1953; Babcock, 1977) on faunal, floral, sedimentologic, and stratigraphic aspects of these units has confirmed the initial conclusions. Never-the-less, considerable controversy exists over whether the Capitan Formation, the second facies mentioned above, represents a "true" or "ecologic" reef. The controversy is well summarized in Cys and others, 1977. Various workers have considered the Capitan to represent an unconsolidated shelf margin skeletal bank, or mound (Lang, 1937; Achauer, 1969), a true barrier reef (Newel1 and others, 1953), or an uninterupted slope facies (King, 1948). Others have felt that the abundance of inorganic, early submarine cement indicated that the wave-resistant nature of the Capitan "reef" was a result of primarily inorganic rather than organic processes making this a "cement reef" rather than an "organic or ecologic reef" (Schmid and Klement, 1971). Basically, the problem boils down to the recognition of in-situ, Erame-building organisms in the Capitan

Formation. If these can be recognized (in quantity), and we believe they can be, then the complex can reasonably be called a reef. The biological diversity of this environment (see table 1); the abundance of framework calcareous sponges, bryozoans, and hydrocorallines; the ubiquitous presence of encrusting organisms (Tubiphytes, Archaeolithoporella, Girvanella, and other groups); the remarkably high productivity of organisms (generating vast masses of reef and fore-reef skeletal debris); the presence of major volumes of inorganic, radial-fibrous, originally aragonitic cements; and the large-scale fragmentation and disruption of fabrics by wave and current activity are all features of the Permian reef complex which are highly analogous to modern reefs. Indeed, much of the semantic confusion over the reef nature of the Capitan Formation is largely a product of the "fair-weather" examination of modern reefs. On a clear, calm day when most geologists venture forth, the modern reef is a truly wave-resistant structure consisting of abundant, in-situ framework organisms. The day after a hurricane, however, much of this "waveresistant framework" has been smashed into rubble which accumulates within the reef or is transported into deeper water settings. Indeed, quarries in Pleistocene or older reefs show only a small percentage of in-place framework organisms coupled with extensive encrustation and submarine cementation of reef debris. 
Each of the major depositional facies of the Permian Basin will be examined during this field excursion and so the other, non-reef facies will not be extensively described here. The generalized facies patterns are shown in figure 2 and Table 1. The back reef area consists of skeletal sand banks, islands, lagoons, and sabkhas. From the farthest back-reef area to the reef these sediments include: nodular gypsum and anhydrite beds interlayered with red siltstones; tan, aphanocrystalline dolomitic mudstone beds with evaporite crystal casts; interbedded thin, laminated sandstone-siltstone units; pure, locally stromatolitic or calcisphere-rich, dolomitized carbonate mudstones; dolomitized pelletal mudstones; dolomitized pisolitic grainstones; partially dolomitized green algal-foraminiferal grainstones; and a very narrow zone of reef-derived, back-reef rubble.

The basinal areas contain turbidites and slumps of reef- and back-reefderived carbonate material. Some of it accumulated as thin sheets of finegrained debris which spread over much of the basin. The bulk of the carbonate debris accumulated near its sources along the margins of the basin (in a few cases reworked into submarine mounds or "1ithoherms" by contour currents). The main volume of basinal sediment is finely laminated sandstone and siltstone also transported from the shelf to the basin by gravity-driven currents.

It must be kept in mind that, although the three rock packages mentioned above are lateral time-equivalents of each other and have approximately the same overall thickness, this equivalence does not necessarily extend to smaller scale units. Thus, laminated sandstones which are tens to hundreds of meters thick in the basin facies may have essentially no equivalents in the reef or back-reef sections. Likewise, reef and back-reef limestone and dolomite sequences which, again, may be tens to hundreds of meters thick, will commonly thin to less than a meter toward the basin center. So although large-scale overall age equivalence is present, we must also think in terms of non-synchronous or "reciprocal" sedimentation on smaller scales (Wilson, 1967; Meissner, 1972). This reciprocal sedimentation may be related to tectonic movements or eustatic fluctuations in sea level which shift the locus of active sedimentation or change the balance of influx of clastic terrigenous versus carbonate sediment.

The abrupt lateral facies changes in these Permian sediments are reflected in the complex stratigraphic terminology which has been applied to these units.

The detailed stratigraphic nomenclature of the Permian Basin will not be discussed here as the terminology of King (1948) and Newell and others (1953) will be followed with only minor modification. Stratigraphic nomenclature, correlations, and age designations for shelf, shelf edge, and basin units are shown in Table 2 and figure 3. 
The Permian Basin region was subdivided during Guadalupian and earlier Permian time into a series of smaller basins and platforms (fig. 1). The orientation of these features was largely controlled by pre-Permian northwestsoutheast oriented faulting of the Ancestral Rockies trend. These early lineaments, still visible in the Sierra Diablo Mountains, were modified by gentle, late Pennsylvanian and early Permian flexures. An even greater modification was produced by differential sedimentation. Original structural relief was significantly accentuated by higher rates of sedimentation of shallow water carbonate deposits on structural "highs" compared with lower rates on structura1 "lows". Thus, basins which were only a few tens of feet deep at the start of Permian time eventually had water depths in excess of $1,500 \mathrm{ft}$ by the close of Guadalupian time.

The three major facies packages mentioned earlier--basin, reef, and backreef--are strictly controlled by these structural sedimentologic features. Basinal facies cover the entire region of Delaware and Midland Basins. Reef facies are discontinuously distributed both in space and time but generally are confined to a very narrow belt bounding the platform areas. The back-reef province, in its broad sense, covers much of the platform areas.

The interrelationships between these facies are governed by a number of factors. Eustatic sealevel stands and(or) relative rates of subsidence versus sedimentation, as mentioned earlier, can lead to "reciprocal" sedimentation patterns. Ecological conditions, such as water temperature, salinity, turbidity, or other factors, can affect reef formation and, thus, overal1 facies patterns. Indeed, just within the Permian facies of the Guadalupe Mountains region, one can see remarkable variations in microfacies patterns. Bank margins of non-reefal bioclastic calcarenite are present in some intervals (Victorio Peak and Getaway units). Reefs which prograde largely horizontally out over reef talus are dominant at other times (upper Capitan unit). Yet other reefs which build up almost vertically in the section form the bank margin in the Goat Seep unit. Finally, terrigenous sand sheets cover the entire region from back-reef to basin at other times. So the discussion of facies patterns in the Permian Basin region must take into account these major variations in modifying factors. This excursion will focus primarily on the upper Capitan interval as this is the best exposed and most intensely studied part of the section. It must be kept in mind, however, that this is just one of a number of facies patterns which can be observed in the region. The climatic setting of the Permian reef complex also had a major influence on both depositional and diagenetic processes. The region lay at the western margin of a broad alluvial plain to the east of the Appalachian area. The basin was presumably connected to a major western and southern ocean by narrow channels (fig. 1). The entire region lay within 10 degrees of the Permian equator and, as evidenced by the extensive back-reef evaporite deposits, clearly had a hot and very arid climate. 
During Guadalupian and earlier Permian time water circulation in the Delaware Basin was apparently adequate to maintain normal marine salinity of the surface water along the bank margins. Waters penetrating deeper onto the banks were evaporatively concentrated to high salinities. Generation of heavy brines on the banks, which periodically flowed into the basin, may have contributed to euxinic, stratified water masses in the deeper parts of the Delaware and Midland Basins. Progressive restriction of the passageways between the Delaware Basin and the "open oceanic" areas to the south and west led to apparent salinity increases and extinction of reef growth in the region at the close of the Guadalupian. Continued aridity, coupled with restricted influx of marine waters led to the deposition of more than 2,000 ft of evaporite sediments in the Delaware Basin, completely filling the topographic depression left after Guadalupian time.

The extreme aridity of the region also had other influences. Transportation of clastic terrigenous debris was dominated by eolian processes. Equilibrium eolian deflation surfaces (sabkhas) are present in back-reef areas and dune migration may have been responsible for transport of a significant volume of sand to the shelf edge from where it could be moved into the basin, especially during low stands of sea level. Aridity also presumably prevented the formation of extensive karstification during sea level drops, allowed the development of widespread "coastal caliche", and led to the formation of hypersaline brines which may have contributed to the extensive dolomitization of back-reef carbonate sediments.

The question of relative sealevel changes, mentioned previously as part of the model of "reciprocal sedimentation", should also be examined further. Regional subsidence, local tectonic effects, eustatic sealevel stands, and epirogenic movements all can play a role in relative sealevel stands. Other factors, such as variations in sedimentation rate, also can yield apparent changes due to progradation or retrogression of shorelines. Numerous authors have pointed out that cyclic sedimentation of one sort or another is widespread in the Permian Basin in Pennsylvanian as well as Permian strata (eg. Meissner, 1969; Silver and Todd, 1969). Cyclic sedimentation operated at a number of scales involving fractions of an inch to hundreds of feet of sediment and were superimposed on an apparently long-term drop in sea level throughout the Late Permian. Known Late Pennsylvanian to Early Permian glaciation may have contributed to some of the cycles by creating periodic eustatic sea level changes. Although dating of these southern hemisphere glacial events is far from exact due to the provinciality and endemism of the floras and faunas present, glaciation is not considered to extend into the Late Permian and thus may not explain Guadalupian cycles and the global regression at the close of Guadalupian time. Epeirogenic events, late orogenic deformation in Appalachian and Hercynian regions, and variations in seafloor spreading may account for Late Permian cycles. Moreover, regional basin subsidence patterns may have been episodic and could also have contributed to the cyclicity of sedimentation. 


\section{Diagenetic Patterns}

Generalized patterns of diagenesis in the Guadalupian section are outlined in figure 2 and table 1 . The back reef area is characterized by the highest average porosities. These areas, which were the topographically highest facies in the complex, were frequently subjected to subaerial exposure and freshwater diagenesis. Evaporitic conditions in restricted lagoons and sabkhas led to the formation of evaporite minerals (gypsum and anhydrite). the withdrawal of these calcium sulphate minerals from the shelfal waters led to elevated $\mathrm{Mg} / \mathrm{Ca}$ ratios and perhaps also to the formation of dolomitizing brines as in the modern Persian Gulf. Alternatively, but less probably, freshwater input and mixing with marine pore fluids may have led to dolomitization through "brine mixing" ("Dorag" dolomite). Thus, the back reef areas of the Permian reef complex are typified by calcareous grainstones and mudstones with a mixture of preserved primary porosity and secondary porosity related to such factors as early freshwater cementation and leaching, early dolomitization, or late (mesogenetic) dissolution of evaporite minerals.

The reef facies has very low average porosities. Small-scale permeability is very low but large-scale permeability is quite high as a result of fracturing. Porosity in this facies was completely obliterated by submarine cements probably within a few tens to hundreds of years after the time of reef deposition. These relatively coarse, radial, fibrous crusts of orginally aragonitic(?) cement formed within open pores in these Permian reefs just as in many modern reefs. Euther porosity destruction was accomplished by the infiltration of muddy, pelletal, internal sediment into remnant pores.

This type of cementation affected not just the reef-crest sediments but extended for several hundred feet down the fore-reef slope (a feature also seen in modern reefs). Thus, the upper fore-reef slope also has very low porosities. The lower half of the fore-reef talus facies has more complex diagenetic relations. Lesser amounts of submarine cementation are seen here. However, medium-crystalline, relatively late, very strongly fabricselective dolomitization is present within this environment and has resulted in the replacement of about $1 / 2$ of the original carbonate material in this facies. The source of dolomitizing fluids may be either from the overlying back-reef facies or from the hypersaline basin waters of Castile and Salado time. Such dolomitization has not led to significant secondary porosity in this facies, however.

The toe of the fore-reef slope is characterized by compaction and silicification. Calcitic fossil fragments, especially brachiopods, bryozoans, and echinoderms, were selectively and delicately silicified by chert, chalcedony, and megaquartz. In some cases, silicification extended to aragonitic fossils or formed non-selective chert nodules which cross-cut primary fabric elements. The source of silica most likely is from siliceous sponges and radiolarians which lived in lower slope and (or) basinal settings.

The basin facies is typified by calcite and very subordinate quartz cementation of sandstones as well as compaction of sandstone, siltstone, shale, and carbonate beds. Porosity in finer-grained basinal sandstones can be quite high (as high as 27 percent; Williamson, 1977, p. 414) with corresponding permeabilities in the tens to hundreds of millidarcies. 


\section{Recent Models}

The Permian depositional and diagenetic patterns described here (and summarized in figs. 2 and 3 and in table 1) can be matched quite closely in some modern settings. The basinal relations can be modeled in the Mediterranean, the Black Sea, and to some degree, in the Bahamas-Florida area. The restricted circulation and partially euxinic conditions can be found, to some degree, in the Red Sea, Mediterranean, and Black Sea during the Tertiary or Quaternary; but the deep, relatively elongate, structurally controlled basins surrounded by steep, reefal escarpments bordering isolated platform areas are best modeled in the Bahamas. The suite of facies from fore-reef debris, reef, back-reef rubble, near-back-reef skeletal sands and muds, islands, restricted lagoons, and finally supra-tidal facies seen in the Permian is remarkably similar to the general facies spectrum found in the Florida Keys-Florida Bay area. Yet climatically and paleogeographically, the Permian of west Texas and New Mexico was much more like the arid, continentinterior southwestern margin of the Persian Gulf than the high-rainfall, ocean-margin region of south Florida. Thus, the lagoons and sabkhas of the Trucial Coast of the Persian Gulf provide an excellent analog for the farback-reef areas of the Permian.

It is clear, then, that no single area today provides a complete or exact analog for the Permian Basin. Yet if we combine the climatic factors of the Persian Gulf with the tectonic-sedimentologic patterns of the Florida-Bahamas region and the hydrographic factors of the Mediterranian-Black-Sea-Red Sea area we can very closely approximate the patterns seen in the Permian.

\section{Dil and Gas Production}

The Permian Basin has had hydrocarbon production for nearly 60 years and is one of the most prolific petroleum provinces in North America. To date, "approximately 91.6 billion barrels of oil-in-place and about 106.2 trillion $\mathrm{cu} f \mathrm{f}$ of dissolved/associated and non-associated gas-in-place have been discovered in the Permian Basin" (Dolton and others, 1979. p. 1). Production from the Permian Basin extends from the Cambrian (Wilberns Fm.) to the Cretaceous (thin carbonate units) although production from units younger than Permian is negligible. Paleozoic reservoirs produce oil from depths of less than 500 to greater than $14,000 \mathrm{ft}$ and also produce gas from depths of less than $500 \mathrm{ft}$ to greater than $21,000 \mathrm{ft}$ (Dolton and others, 1979). The Permian section is mainly oil productive with greater than 65 billion barrels of oilin-place ( 71 percent of the total discovered in the Permian Basin) having been discovered to date (in 2,188 pools). Non-associated gas production, on the other hand, comes predominantly from pre-Mississippian strata. Permian units contain only about 6.3 trillion cu ft of non-associated gas-in-place (about 13 percent of the total for the Permian Basin). However, Permian units contain 32.7 trillion cll ft of associated/dissolved gas-in-place (54 percent of the Permian Basin total) (Dolton and others, 1979).

The predominance of oil production from Permian units is clearly related to their relatively shallow burial in this region where virtually all Permian strata are found at present-day burial depths of less than 15,000 ft. Furthermore, virtually all production from Permian rocks comes from units at less than 10,000 ft burial depths; most of it from less than 5,000 ft depths (Dolton and others, 1979). 
"The four provincial series of the Permian do not contain hydrocarbons in equal amounts. The largely evaporitic Ochoan rocks have accounted for only about 6 million bbls of discovered oil in-place, less than 0.01 percent of the Permian's 65 billion bbls" (Dolton, and others, 1979, p. 24).

"By contrast, the Guadalupian has accounted for 67 percent of all Permian oil found and 62 percent of all Permian gas. The Leonardian follows with 28 percent of the oil and 32 percent of the gas. The Wolfcampian contains 5 percent of the oil and 10 percent of the total Permian gas. These amounts are directly related to the progressive development of reefs and back-reef lagoons beginning in the Wolfcampian, increasing in the Leonardian, and culminating in the development of the Capitan reef complex in the Guadalupian.

Hydrocarbon traps in Permian rocks are largely a combination of stratigraphic and structural types, although each type does occur alone. the intricate stratigraphic interfingering of lithologies responsible for trapping much of the Permian oil has resulted largely from the constantly shifting... sedimentary environments. Primary sealing mechanisms are porosity and permeability barriers of carbonate, evaporite or shale.

About 40 percent of the [Permian] reservoirs are limestone, 29 percent are dolomite and 29 percent are sandstone. Porosities range from 1.5 to 25 percent and reservoir permeabilities from 0.02 to 200 millidarcies.

Recovery factors range from a low of 7.6 percent to a high of 47.5 percent. The fractured siltstone Spraberry reservoir of the Midland Basin has a very low recovery factor, although the volume of oil in-place is the largest of any single Permian pool. The average recovery factor for the Permian System is 25 percent." (Dolton and others, 1979, p. 24).

Detailed production (not reserve) figures for oil and gas fields developed in selected Leonardian, Guadalupian, and Ochoan units are shown in Table 3 (listed by county). Production totals for each producing stratigraphic unit are given in Table 4 along with a grand total for all these strata in the Permian Basin region. Only units which will be seen on this field trip have been included in these tables. Extensive production from ageequivalent but differently named units from the Central Basin Platform, Midland Basin, and Eastern Shelf have not been listed. Data for these tables was supplied by the Petroleum Data System, University of Oklahoma, Norman, Oklahoma.

Even a cursory examination of these tables will show that there is no production from the Capitan, Victorio Peak, or Goat Seep reef or fore-reef facies which were tightly cemented at the seafloor shortly after deposition. The vast bulk of production (greater than 90 percent) is from primary or early diagenetic secondary porosity in back reef dolomites and sandstones of the Tansill, Yates, Seven Rivers, Queen, and Grayburg Formations or the open shelf facies of the San Andres Limestone. A second, much smaller, peak of production comes from channel sandstones of the Delaware Mountain Group (particularly in the Bell Canyon Fm.) and basinal limestones of the Bell Canyon Fm. or Bone Spring Limestone. More significant oil reserves in basinal sandstones are found in the Midland Basin. There, the Spraberry Fin. has more than 8 billion barrels of oil-in-place. However, recovery factors of less than 10 percent indicate ultimately recoverable reserves of about 534,000,000 barrels of oil. 
Individual channels in the "Ramsey Interval" near the top of the Bell Canyon Fm. are up to $100 \mathrm{ft}$ thick, $1 / 4$ to 4 miles wide, and 50 miles in length (Williamson, 1977). These channels have a very pronounced regional trend (NESW for the "Ramsey") which strongly controls the shape and distribution of basinal oil fields.

In this setting, then, back-reef environments account for greater than 90 percent of all hydrocarbon production with basinal sediments accounting for the rest. Reef and fore-reef facies are totally non-productive. Clearly, penecontemoraneous and early burial diagenesis played a major role in controlling the distribution of reservoirs. Evaporite formation and dissolution, synsedimentary dolomitization, early vadose and phreatic leaching and cementation, coupled with probable early oil migration from rapidly deposited and buried, overpressured source rocks in the basins, led to outstanding reservoir characteristics on the shelf. Early submarine cementation obliterated reservoirs on the shelf edge and slope long before oil migration. Finally, some basinal reservoirs may have been preserved from compactional porosity loss by overpressuring beneath 2,000 or more feet of rapidly deposited evaporites.

The source for most of this Permian oil is presumably from the euxinic, relatively organic carbon-rich, basinal sediments such as the Bone Spring Limestone and some intervals within the Delaware Mountain Group. Although these units generally have organic carbon contents of less than 1 percent (King, 1948; Palacas, oral commun., 1978), their carbonate composition, great thickness, and intervening sandy, permeable zones may mean that they can act as very efficient source rocks. Dil reservoired in the basinal facies, then, has probably migrated only a short distace from source to reservoir. Much of the oil in the back-reef sections, however, presumably moved upsection or laterally through fractured reef sediments to get from source to reservoir. The fracturing of the reef was essentially contemporaneous with deposition (because of compaction of the thick, underlying reef talus) and thus, even syndepositional reef cementation probably did not significantly retard fluid movement. Indeed, even today, the tightly cemented reef zone has the highest permeability of any of the Guadlaupian bank-to-basin facies (Motts, 1968).

Current estimates of the volume of undiscovered hydrocarbons in-place for Permian rocks of the Permian Basin are that "at the 95 and 5 percent probabilities, 1.0 to 6.0 billion bbls of oil in-place ( 1.5 to 9.2 percent of the discovered Permian crude oil) remain undiscovered, while 0.7 to 4.1 trillion cu ft of dissolved/associated gas in-place (2.2 to 12.4 percent of the discovered dissolved/associated gas) remain undiscovered. Finally, 0.2 to 0.6 trillion cu ft of non-associated gas in-place (3 to 21 percent of the discovered non-associated gas) remain undiscovered. Most of these undiscovered in-place hydrocarbons occur above 10,000 ft" (Do1ton and others, 1979, p. 47).

"These undiscovered amounts will probably occur in circumstances similar to known fields and pools with respect to reservoir characteristics, seals, source beds, and nature of the hydrocarbons. Traps will probably be predominantly stratigraphic. The undiscovered deposits are likely to be distributed in undrilled areas surrounded by or flanking known production. Such flanking areas are in the western part of the Northwestern Shelf, the western areas of the Delaware Basin, and the southern and western parts of the Val Verde Basin." (Dolton and others, 1979, p. 47). 
Studies have shown that "undiscovered pool sizes are small; only at the 5 percent probability is there a chance of occurrence of an oil pool of 16 million bbls or larger, or a non-associated gas pool of 24 billion cu ft or larger." (Dolton and others, 1979, p. 47).

\section{Concluding Notes}

For further general discussions of Permian Basin depositional and diagenetic facies patterns the papers by King (1948), Newell and others (1953), Hayes (1964), and Cys and others (1977), are recommended. Other, more specific papers, can be found in the extensive bibliography on the Permian Basin region given at the end of this section of the guidebook.

Further discussions of the specific details of facies patterns and diagensis are also presented in the roadlog section of this guidebook. The $\log$ is based, in large part, on preexisting guidebooks (Nelson and Haigh, 1958; West Texas Geological Society, 1960 and 1969; Hobbs, Roswell, and West Texas Geological Societies, 1962; Roswell Geological Society, 1964; Dunham, 1972; Pray, 1975; and Pray and Esteban, 1977). However, this guidebook has extensive additional commentary on many localities and is organized differently from previous guides. All roadlogs are based on continuous routes with side trips being presented as separate, supplementary logs. Thus, the trip from El Paso to Carlsbad is logged as a continuous route with the excursions to McKittrick Canyon, Walnut Canyon, and Dark Canyon-Rocky Arroyo being listed as separately logged routes. This adds complexity to a bus tour but makes the logs much easier to use on car trips. Furthermore, for ease of use, all figures are in a single section after the roadlogs. Roadlog routes are shown in fig. 4. A generalized geologic map of the Guadalupe Mountains area is presented in Plate 2 (in pocket). 
EL PASO TO CARLSBAD ROADLOG

$\begin{array}{ccc} & \text { Cumul. } & \text { Mileage } \\ \text { From } & \text { From } \\ \text { Mileage } & \text { E1 Paso } & \text { Carlsba } \\ 0.0 & 0.0 & 159.4 \\ 0.6 & 0.6 & 158.8 \\ 6.5 & 7.1 & 152.3\end{array}$

Description

Leave Caballero Motel; turn right (heading east) on U.S. Highway 62 and 180 (Montana Avenue).

Intersection with Airway Road (airport about $1 / 2 \mathrm{mi}$ to left); continue straight ahead.

Stabilized (vegetated) dunes of clastic terrigenous debris blown from the floor of the Hueco Bolson, the flat, intermontane basin we have been and are continuing to cross. The Hueco Bolson is one of the southernmost grabens of the Basin and Range Province and is bounded on the west by the Franklin Mountains and on the east by the Hueco and Sacramento ranges. To the north, near the Texas-New Mexico border, the the Hueco Bolson is separated from the Tularosa Basin (which has completely internal drainage) by a low ridge. The Hueco Bolson has external drainage along its southeastern side through the Rio Grande River valley. The average elevation of the Hueco Bolson is approximately 4,000 $\mathrm{ft}$, and the basin averages $25 \mathrm{mi}$ in width and $80 \mathrm{mi}$ in length. "A recent (1967) USGS seismic and gravity profile across the Hueco Bolson from the base of the Franklin Mountains to the base of the Hueco Mountains indicates a deep structural trough bounded on the west by a large normal fault. The maximum thickness of the Hueco Bolson fill in the center of this trough is calculated to be about 9,000 feet." (McGlasson and Seewald, 1969). The bolson fill ranges in age from Miocene to Holocene; the Pleistocene deposits are particularly thick, with local accumulations of as much as $5,000 \mathrm{ft}$ of Pleistocene alluvial and lacustrine sediment (Strain, 1969).

$\begin{array}{llll}1.5 & 8.6 & 150.8 \quad \text { E1 Paso city limit. }\end{array}$

$3.1 \quad 11.7 \quad 147.7$ Junction with Texas FM Road 659 to Ysleta (on right) and start of two-lane section of U.S. Highway 62 and 180; continue straight ahead. The intensely blockfaulted, low-relief (ca. 1,000-1,500 ft) Hueco Mountains can be seen directly ahead. Precambrian to Tertiary igneous, metamorphic and sedimentary rocks are exposed in this range (see fig. 5). Cerro Alto, the highest peak, is a Tertiary syenite porphyry intrusive. The Permian (Wolfcampian to early Leonardian) section of the Hueco Mountains contains nearly 2,000 ft of limestone and very subordinate shale. The pre-Permian sedimentary section consists of a lower interval of Cambrian to Mississippian strata (mainly limestones and dolomites) and an upper interval of Pennsylvanian limestones, shales, 
and subordinate sandstones. The upper and lower intervals each contain up to $2,000-4,000 \mathrm{ft}$ of section. The lower interval shows disconformable contacts typical of stable shelf sections, whereas the upper interval contains angular unconformities indicative of the increased tectonic activity and bank-to-basin differentiation of that time period.

Throughout this trip, the bank-to-basin facies sequences we see will be related in one way or another to the initial, structurally controlled topographic variations generated during this Pennsy1vanian and Permian deformation. The folding and faulting of this period, part of the Ancestral Rockies movements, generally trends northwestsoutheast. The Diablo Platform, Orogrande Basin, Pedernal High, Northwest Shelf, and Delaware Basin (fig. 1) were among the many major physiographic features formed during this time interval. This primary structural relief was, in most cases, strongly modified by subsequent differential sedimentation.

$4.4 \quad 16.1 \quad 143.3$

$2.5 \quad 18.6 \quad 140.8$

$0.4 \quad 19.0 \quad 140.4$

$0.8 \quad 19.8 \quad 139.6$

$0.6 \quad 20.4 \quad 139.0$

$0.3 \quad 20.7 \quad 138.7$

$0.3 \quad 21.0 \quad 138.4$
Loose (unvegetated) dunes deposited by winds from the northwest which lose velocity and drop sand near the base of the Hueco Mountains. The hills on the right (largely covered by dunes) are made of Wolfcampian limestones of the Hueco Group.

Quarry in Hueco Group limestone on the right. The limestone here is about 97 percent pure $\mathrm{CaCO}_{3}$ and the quarry exposes a single, thin, fusulinid-rich bed about $3 / 4$ of the distance up the rock face.

Lower part of Hueco Group is exposed in hill on right. The unconformable contact between the Hueco limestone and the underlying lower part of the Pennsylvanian Magdalena Limestone is exposed in the low knob at the base of the eastern slope of the hill. This unconformity is an indication of pre-Permian uplift and erosion on the Powwow anticline.

Exposures of the Mississippian Helms and Pennsylvanian Magdalena Formations in hills on left.

Quarry at 2:00 $0^{\prime}$ clock in Magdalena Limestone The very pure ( 99.8 percent $\mathrm{CaCO}_{3}$ ) limestones in this quarry, as well as those from the quarry at mile 18.6, were formerly transported to El Paso where they were calcined to $\mathrm{CaO}$ (quick lime). Junction with Texas SF Road 2775 to Hueco Tanks State Park; continue straight ahead.

Hills on left capped by Magdalena Limestone. The southeast dips visible here are on the flank of the Powwow anticline. The Jones No. 1 Sorley well, drilled a few miles west on the crest of the Powwow anticline, encountered Precambrian granites at 2,172 ft depth. Helms Peak (elevation 5,409 ft) at 2:00 o'clock is capped by 1imestones of the Hueco Canyon Formation unconformably overlying middle 


$\begin{array}{lll}0.7 & 21.7 & 137.7 \\ 1.9 & 23.6 & 135.8 \\ 0.5 & 24.1 & 135.3 \\ 0.8 & 24.9 & 134.5\end{array}$

$0.4 \quad 25.3$

$0.3 \quad 25.6$

134.1

133.8

Magdalena Limestone.

Entering Powwow Canyon.

Road cut in middle Magdalena Limestone.

Road cut in middle Magdalena Limestone.

Roadside park on left. The unconformity at the base of the Permian is clearly visible directly ahead. On the western side of the Hueco Mountains this unconformity cuts down to the Ordovician El Paso Limestone. In the outcrop directly ahead, the unconformity is between the Magdalena Limestone and the basal Hueco Group.

Road cut in upper $130 \mathrm{ft}$ of Magdalena Limestone. Road cut in the basal part of the Permian Hueco Group (including the poorly exposed Powwow Conglomerate and the overlying upper member of the Hueco Canyon Formation). The Powwow Conglomerate varies locally in thickness but is about $30 \mathrm{ft}$ thick in this area. It contains red shales, siltstones, and chert- and limestone-pebble conglomerates. Another redbed interval (the Deer Mountain Shale) occurs near the top of the Hueco Group. These redbeds are considered to be the southern tongues of the thick, predominantly redbed Abo Formation of the Sacramento Mountains.

$\begin{array}{lll}0.2 & 25.8 & 133.6 \\ 1.2 & 27.0 & 132.4 \\ 8.7 & 35.7 & 123.7 \\ 3.1 & 38.8 & 120.6\end{array}$

Road cut in Hueco Canyon Formation, mainly shelfal limestones.

Hueco Inn on left. Continued Hueco Group outcrops for next twelve miles.

Forty Mile Hill (elevation 5,427 ft). Leaving Hueco Mountains; emerging onto Diablo Plateau.

Roadside rest area on left with view of numerous extrusive and intrusive igneous features to the north and northeast in the Cornudas Mountains. The central, high volcanic cone is San Antonio Peak ( $7,020 \mathrm{ft}$ ).

$\begin{array}{rrr}13.3 & 52.1 & 107.3 \\ 4.7 & 56.8 & 102.6 \\ 0.5 & 57.3 & 102.1\end{array}$

Limestones of Hueco Group in road cut.

Road on Lower Cretaceous Campagrande Formation.

Junction with Texas Ranch Road 2317; continue straight ahead. Molesworth Mesa, which lies to the south, is composed of Cretaceous Trinity and Fredricksburg sediments.

$1.9 \quad 59.2 \quad 100.2$

Mountains at 9:00 $0^{\circ}$ clock are the Sierra Tinaja Pinta, a breached laccolith. The anticlinal structure in the center is composed of Bone Spring Limestone and is flanked by sediments of the Yeso Formation.

$\begin{array}{lll}5.0 & 64.2 & 95.2 \\ 6.1 & 70.3 & 89.1\end{array}$

Junction with Texas Ranch Road 1111; continue straight ahead.

The Antelope Hills, containing basal Cretaceous sandstones cut by a Tertiary sill, are visible at 3:00 o'clock.

$\begin{array}{llll}2.7 & 73.0 & 86.4 & \text { Road is on Bone Spring Limestone. } \\ 1.5 & 74.5 & 84.9 & \text { Junction }\end{array}$

Junction with Texas Farm Road 1437 to De11 City on left; continue straight ahead. Dell City is a farming community in the Salt Flat Bolson which has grown up as a consequence of the development of wells 


$\begin{array}{lll}0.1 & 74.6 & 84.8 \\ 6.5 & 81.1 & 78.3 \\ 1.0 & 82.1 & 77.3 \\ 0.25 & 82.35 & 77.05\end{array}$

drawing Pleistocene(?) ground water from the bolsonfill. Rapid depletion of the water supply (use exceeds recharge) and increasing soil salinites indicate a short or very expensive continued existence for agriculture in this region.

Road crosses basal Cretaceous sandstones and passes into Bone Spring Limestone.

Los Alamos Hills can be seen to the south of the highway, in foreground. Leonardian to basal Guadalupian rocks are exposed here.

Junction with Texas Fr Road 1576 to De11 City. Turn left for view stop.

STOP I-1. This location provides an excellent view of the Guadalupe and Delaware Mountains and the Salt Flat Bolson (in which we are now standing). We can see a magnificent panorama including the Upper Permian section of the Guadalupe Mountains, about 20 miles to the northeast. This $5,000 \mathrm{ft}$ escarpment is formed by a major north-south trending normal fault system which marks the eastern boundary of the Salt Flat Bolson. To the northwest and west we can see the Cornudas Mountains, Cerro Diablo, and Sierra Tinaja Pinta, a series of Tertiary igneous plugs and lava flows. To the south lies the Sierra Diablo range which is terminated by the Babb flexure zone, a monocline, at its northern end. Upper Permian (Guadalupian) limestones and sandstones of the Cherry Canyon Formation compose the two mesas to the north of the Babb flexure. Also visible beyond these mesas is Sierra Prieta, another Tertiary intrusive.

Finally, to the southeast, Jpper Permian strata, primarily Brushy Canyon Formation basina1

sandstones, are visible in the face of the long Delaware Mountain escarpment.

Because the Guadalupe Mountains are the major focus of this portion of the field trip, let us take a closer look at that range (fig. 6). Although the topography of the eastern side of the Guadalupe Mountains is controlled almost entirely by the undeforined primary facies distribution of the Guadalupian sediments, the western face is completely controlled by Tertiary normal faults. Thus, on the on the western side, strata with a northeast-southwest facies strike are obliquely transsected by a north-south trending fault zone. Furthermore, we are viewing the exposure obliquely which makes accurate geologic observation even more difficult.

For reference purposes let us name the major peaks on the Guadalupe Mountain skyline. From south to north these include the massif of El Capitan (elevation 8,078 ft), Guadalupe Peak (the highest point in Texas at $8,751 \mathrm{ft})$, Shumard Peak $(8,626 \mathrm{ft})$, an unnamed spur off Shumari Peak (about 8,350 ft), Bartlett Peak $(8,513 \mathrm{ft})$, and Bush Mountain $(8,676 \mathrm{ft})$. 
The massive, light colored rocks, which compose the upper parts of $\mathrm{E} 1$ Capitan, Glladalupe, and Shumard Peaks, are Upper Permian (Guadalupian) Capitan limestones and dolomites (see fig. 6 and table 2). Most of this mass is thick-bedded, fore-reef talus (largely of Rader age) which dips steeply (up to 35 degrees) to the southeast, into the basinal sediments of the Delaware Basin. The top of Guadalupe and nearby peaks, however, have true Capitan reef facies and even back-reef sediments. The Capitan reef and fore-reef strata undoubtedly originally extended several miles further south in this region but have been trimmed back by subsequent erosion.

To the north, Bartlett Peak is capped by the oldest exposed Capitan reef limestones which overlie rubble of an older (Goat Seep) reef. The area to the north of Bush Mountain contains the main reef-massif of the Goat Seep as well as age equivalent back-reef calcarenites and terrigenous sandstones (Queen and Grayburg) which stand out clearly as vegetated zones on the mountain slope. "At Guadalupe Peak the smooth slopes below the Capitan are Cherry Canyon and Brushy Canyon sandstones. North of Shumard Peak the upper part of the Cherry Canyon grades into Goat Seep reef. A tongue of Cherry Canyon sandstone continues northward under the Goat Seep reef and grades into small reefs and reefy lime banks in the southern Brokeoff Mountains, and into bedded back-reef rocks in the central Brokeoff Mountains. There Boyd has measured approximately 600 feet of beds which he calls the San Andres formation, and Frenzel, considers to be lower San Andres . .

The rugged cliffs outcropping below the Delaware sand slopes are cut from the dark-bedded Bone Spring limestone of Leonard age. Between El Capitan and Shumard Peak the top of the Bone Spring limestone rises over 1000 feet and this is the Bone Spring flexure described by King. Below Shumard Peak the upper part of the Bone Spring has changed to the gray Victorio Peak . . , a reefy lime-bank facies. The Brushy Canyon sandstone onlaps the Bone Spring flexure and is absent in the slope below Bartlett Peak" (West Texas Geological Society, 1960, p. 50).

The bulk of the strata just described represent just two major phases of basinal progradational filling (Pray, 1975). The Victorio Peak Dolomite and the underlying Bone Spring Limestone form the older (Leonardian) phase. This sequence represents at least two to three miles of basinal infilling and progradation of shelf facies during the accumulation of about 1,000 ft of section (McDaniel and Pray, 1967). "The Leonardian bank margin was eroded in latest Leonardian and/or in early Guadalupian time, 
and a major transgression of basin facies dark carbonates (Cutoff shaly member of King) brought basinal environments far to the north, overlapping Leonardian basin, basin margin, and shelf deposits alike" (Pray, 1975 , p. 5). Presumably this transgression was a consequence either of major regional subsidence or of eustatic sea level rise, perhaps associated with the latest stages of Permian glaciation.

This transgression was followed by the second major cycle of progradation, represented by Goat Seep and Capitan reef complexes. These units built the Guadalupian shelf edge outward several miles by filling in a basin of 1,000 to $1,800 \mathrm{ft}$ depth with steeply dipping, reef-derived debris beds which are clearly visible at the southern end of the Guadalupe escarpment. It is the upper part of this progradational sequence which will occupy much of our attention on this trip.

Turn around and return to U.S. Highway 62 and 180 .

$\begin{array}{lll}0.25 & 82.6 & 76.8 \\ 0.8 & 83.4 & 76.0\end{array}$

$2.7 \quad 86.1 \quad 73.3$

$1.5 \quad 87.6 \quad 71.8$
Turn left on U.S. Highway 62 and 180.

Crossing northern extension of East Diablo fault with the Salt Flat basin down-thrown on the eastern side.

Historical marker near site of former spring-fed oasis (Crow Springs) and a relay station of the Butterfield Overland Stage. The Butterfield route (see cover illustration) was established in 1858 and passed through the area of Guadalupe Pass (Pine Springs). This route lasted only about one year, however, before the entire line was shifted south to the approximate course of the present Interstate 10 through the Davis Mountains. OPTIONAL STOP. Center of playa area of Salt Flat. This major graben, the easternmost of the Basin and Range province, formed in middle to late Tertiary time. The basin is about $60 \mathrm{mi}$ long and $10 \mathrm{mi}$ wide and has been the site of continuous alluvial, fluvial, and lacustrine sedimentation since the middle Tertiary. The thickness of sediments in the basin probably is many thousands of feet. Basin margin sediments include coarse gravels and sands alternating with clays derived from the weathering of the adjacent mountains (especially the Guadalupe and Sierra Diablo ranges). Because there is no natural outlet for the basin, all drainage is internal and sediments become finer-grained toward the basin center. Several important aquifers are present within the basin fill and these are currently being exploited for irrigation in areas such as Dell City to the northwest.

Modern saline playas occur in the Salt Flat area of Texas and the Crow Flat area of New Mexico and Texas, in the topographically lowest parts of the basin (elevations about $3,630 \mathrm{ft}$ ). These playas form in a region of low rainfall (about 9 or $10 \mathrm{in} / \mathrm{yr}$ average) and high evaporation (about $80 \mathrm{in} / \mathrm{yr}$ ) 
(Dunham, 1972). Thus, groundwater, which stands at a level near the playa surface, is drawn upward and is evaporated, leading to gradually increasing salinities. These high salinities greatly restrict vegetation and allow eolian deflation of the rather fine-grained playa preciptates.

Pits excavated in the playa sediments reveal firm but not hard, fine-grained, laminated, non-fossiliferous lacustrine materials. Modern gypsum and minor halite are the dominant evaporite minerals but calcite, aragonite, and dolomite have also been found in the playa sediments either as primary or secondary minerals (see Friedman, 1966; and Dunham, 1972, for further details). $\mathrm{C}^{14}$ age dating and geological mapping of basinal units indicates that much of the sediment found at the surface today may be relict from a larger Pleistocene pluvial lake (King, 1948; Dunham, 1972). Eolian deflation has piled up some of of these primary and secondary minerals as dunes along the margins of the playa area.

Halite has been mined from the surface of the playa in areas to the south of the road although halite is not preserved to any extensive degree in buried sediments. This salt was a most highly valued commodity in the 1880's and was used for food preservation, final curing of hides, and other purposes. It was such a valuable substance that it was hauled by mule- and ox-drawn vehicles for many hundreds of miles over the southwest trail to Fort Quitman, then to San Elizaro, Franklin (now E1 Paso), Paso del Norte (now Juarez), and on to Chihuahua City. Disputes between Mexican and American mining interests in the area led to the El Paso Salt War of 1877. The conflict culminated in the battle of San Elizaro (then the county seat of E1 Paso County). Improved(?) food preservation techniques and more economical sources of salt have eliminated the relatively small-scale mining in this area.

On the south side of the road the Paso Tex oil pipeline can be seen sitting on trestles; on the north side of the road the E1 Paso Natural Gas pipeline to the Pacific Coast is buried beneath playa sediments.

$0.8 \quad 88.4 \quad 71.0$

Gypsum-bearing dunes are derived from deflation of the nearby playa surface.

$\begin{array}{lll}2.2 & 90.6 & 68.8 \\ 0.8 & 91.4 & 68.0\end{array}$

Eastern edge of the Salt Flat Basin.

Folded and downfaulted blocks of Upper Permian on the left.

1.2 92.6 66.8 Beacon Hill on the left is composed of Capitan Limestone with Rader Limestone at the base of the hill. This is the southern end of the Patterson Hills which consist of complexly faulted and folded Upper Permian limestones. Farther north are the Brokeoff

Mountains. 


$\begin{array}{lll}0.5 & 93.1 & 66.3 \\ 0.3 & 93.4 & 66.0 \\ 0.4 & 93.8 & 65.6 \\ 0.3 & 94.1 & 65 . \\ 1.0 & 95.1 & 64.3 \\ 0.8 & 95.9 & 63.5 \\ & & \\ 0.6 & 96.5 & 62.9 \\ 0.5 & 97.0 & 62.4 \\ 0.3 & 97.3 & 62.1\end{array}$

E1 Paso Natural Gas Co. Guadalupe Compressor station on the left.

Road cut in Bell Canyon Formation.

Road cut in Be1l Canyon Formation.

Road cut in Cherry Canyon Formation; note greenish bentonite beds.

Road cut in downfaulted Bell Canyon Formation.

Road cut in Cherry Canyon Formation. The Patterson Hills to the left are capped by Capitan-age limestone.

Road cut in Cherry Canyon Formation exposing contact with Brushy Canyon Formation at east end of outcrop. Junction with Texas Highway 54 to Van Horn on right. Keep left for Carlsbad.

OPTIONAL STOP. An excellent view of the south end of the Guadalupe Mountains here shows the clear relations between reef, fore-reef, and basinal facies (fig. 7). Ahead and to the right, is the escarpment of the Delaware Mountains composed of Brushy Canyon and Cherry Canyon basinal sediments mainly sandstones and siltstones). The Delaware Mountain ridge is capped by the resistant Getaway Limestone which is exposed near the radar station visible at the top of the cliff. The Getaway Limestone Member occurs at a level about 100-200 ft above the base of the Cherry Canyon Formation and, in this area, is a very fossiliferous, gray to black limestone. Note the abundance of lenticular, channelized bedding in the Brushy Canyon and basal Cherry Canyon sections exposed in the Delaware Mountains escarpment. This entire sequence was apparently deposited in water depths of at least several hundred feet by a combination of density currents, mass flows, localized slumps, and perhaps even contour currents. Much of the darker material visible in the cliff face represents relatively fine-grained overbank or nonchannelized flows. The lighter-colored, lenticular sediments are coarser-grained, massive, channe1fill sandstones. Finally, at the very base of the escarpment, a dark ledge of the basinal Bone Spring Limestone is exposed.

To the left we can see the great mass of Capitan Limestone which forms the upper part of E1 Capitan. The Capitan Limestone in this face represents a forereef rubble facies which is the time equivalent of the Hegler, Pinery, and Rader Members of the Bell Canyon Formation. Below the massive $(1,000-1,500 \mathrm{ft}$ thick) limestone, gently sloping deposits of the Cherry Canyon Formation are evident. "The three distinctly visible ledges represent in ascending order the three basinal limestone tongues of this formation named Getaway, South We11s, and Manzanita" (K. W. Klement, in West Texas Geological Society, 1969, p. 18). The rest of the lower slopes of E1 
Capitan are composed of Brushy Canyon Formation.

The lateral flattening of dips from El Capitan to the Delaware Mountains is not a result of deformation, but rather represents a primary facies transition from reef to basin. The thick, steeply dipping, rubbly limestones of the fore-reef facies thin rapidly toward the basin so that 100 or more feet of reef limestone may have a time equivalent $1-5 \mathrm{ft}$ thick limestone in the center of the Delaware Basin. The color change from the light colored, largely oxidized beds of the reef and fore-reef to the dark colored, organic carbon-rich, anoxic basinal facies can also be seen in these exposures.

It should be pointed out that the great thicknesses of basinal sandstones (about 3,500 ft of Delaware Mountain Group), although derived from the shelf, have only thin equivalents in the back-reef facies and are virtually absent in the reef itself. In part, this may reflect the fact that much of the basinal sandstone was apparently transported from the north and north-east, across the Central Basin Platform and the then-filled Midland Basin from sources in the Pedernal Massif and the Arbuckle and Wichita uplifts (Watson, 1979; Bozanich, 1979). Thus, much of the sand influx may have been funneled through gaps in the reef in areas not currently exposed in outcrop. There is evidence, however, from submarine channel orientation, other current direction indicators, stratigraphic relations of basinal sandstones and fore-reef carbonate rocks, and the presence of numerous, if thin, back-reef sandstone beds, that some sand moved across the northwestern part of the basin margin along which we are now standing.

Thus, in all probability, another explanation must be sought for the inverse relationship of thick carbonate and thin sandstone units on the shelf and thin carbonate and thick sandstone units in the basin. Silver and Todd (1969) and Meissner (1972) proposed similar concepts of reciprocal sedimentation to explain these observations. This model suggests that the carbonate sediments were deposited during high eustatic sea level stands. During these times of strong surface-water circulation, reefs flourished and climates were arid. The shelf areas had massive carbonate buildups which maintained the shelf edge at or near sea level. During this time, terrigenous sands were largely trapped in back-reef lagoons or in continental basins. The evaporitic conditions led to the formation of saline bottom-waters and a densitystratified basinal water column. This, in turn, led to largely euxinic, sediment starved conditions on the floor of the basin.

During lowered sea level stands, on the other hand, 
$1.4 \quad 98.7 \quad 60.7$

$\begin{array}{lll}0.4 & 99.1 & 60.3 \\ 0.5 & 99.6 & 59.8\end{array}$

$1.6 \quad 101.2 \quad 58.2$ eolian and fluvial(?) transport of large volumes of we11-sorted arkosic sand to the shelf edge provided a massive supply of unconsolidated material on the upper slope. From there the sands were reworked into the basin by a variety of gravitationally driven current mechanisms. Subsequent transgression in the next cycle removed virtually all traces of sand from the tightly cemented shelf-edge limestones.

Thus, in the reciprocal sedimentation concept, although there is overall time-equivalence of the shelf and basin sediments, they are not exact timeequivalents when looked at in detail. The thick, basinal sandstones are equivalent, in most cases, to hiatuses in reef deposition and to thin sandstone beds in back-reef areas. Thick reef and back-reef limestones are equivalent to very thin, black limestones in the basin.

STOP II-2. This outcrop, faulted at its northern end, exposes Bone Spring Limestone in its basinal facies. This Leonardian limestone, the oldest unit exposed in the Guadalupe and Delaware Mountains, reaches at least $1,700 \mathrm{ft}$ thickness in this area (King, 1948). A thickness of $3,123 \mathrm{ft}$ has been measured for the combined Hueco Limestone-Bone Spring Limestone interval in the Updike well near El Capitan. This combined section thickens to greater than 4,500 ft in the Delaware Mountains to the south (King, 1948, p. 13). At this locality we can see typical, dark gray to black, cherty, interbedded limestones and calcareous shales which are the dominant lithology of the basinal part of the Bone Spring Limestone. Fossils, especially small ammonites, can be found at this locality, but are generally restricted to isolated, granular or calcarentic beds. Bedding surfaces in the basinal Bone Spring Limestone are typically wavy. Both the limestone and the shale units contain considerable amounts of organic matter and may have acted as source rocks within this basin. Indeed, trapped oil can be found even on outcrop in small cavities in the Bone Spring Limestone.

Road cut in Brushy Canyon sandstone.

- Well exposed, lenticular, sandstone channel deposits of the Brushy Canyon Formation are visible in the distance on both the left and right sides (fig. 8).

STOP II-3. An exposure of basinal Brushy Canyon Formation, the lowest unit in the Delaware Mountain Group. The feature of special interest at this locality is the exposed margin of a submarine channel (fig. 9). Such channels are common in this formation and, at least in this area, generally trend northwest-southeast, that is, perpendicular to the shelf margin. 
At this locality, we can see dark-colored, graded, relatively fine-grained sandstones, siltstones, and shales in thin beds with some soft-sediment deformation features. These àre abruptly cut by a uniform, thick-bedded, sandstone-filled channel. Both types of sediments were clearly soft, even fluid, at the time of deposition, as shown by the fact that the channel margins are extensively deformed by sand injection.

Hayes (1964), Jacka and others (1968), Payne (1979), Berg (1979) and other authors have interpreted these or similar deposits as submarine fans. The graded, finer-grained sediments are considered to be interchannel or overbank turbidity-current deposits. The cut and fill, massive sandstones are interpreted as part of an anastomosing system of fan channels, eventually abandoned or filled by sand transport. Harms (1974), on the other hand, proposed that the finer-grained sediments were deposited by density overflows which dropped suspended sediments as they moved out over density interfaces within the water column rather than at the sediment-water interface. The channels were cut, according to Harms, by saline and cold density currents (rather than turbidity currents) which formed on the shelf. The sand fillings of the channels were also laid down by density currents.

In either case, these large (commonly more than $1 / 2$ mile wide and 50-100 ft thick) channelized sandstones, surrounded by lower permeability siltstones and shales, represent significant potential stratigraphic traps. This is especially true because of the close spatial association of these sandstones and the potential basinal source rocks. Indeed, exploration efforts to date have located more than 100 oil and gas Eields which produce from channe1-sandstone reservoirs of the Delaware Mountain Group, primarily (but not exclusively) from the Bell Canyon Formation (see table 3).

$0.5 \quad 101.7 \quad 57.7$ Roadside rest areas on left and right. Fxcellent views of E1 Capitan (fig. 10) and the Delaware Mountains escarpment. Again, the bank-to-basin transitions of the Capitan Limestone and its equivalents are well shown.

$0.7 \quad 102.4 \quad 57.0 \quad$ Road cuts in sandstones and siltstones of the Brushy Canyon Formation.

$0.4 \quad 102.8 \quad 56.6$ OPTIONAL STOP. View along the Delaware Mountains escarpment on the right. Outcrops on the left consist of upper Brushy Canyon Formation sandstones with oriented fusulinid Coraminifera (fig. 11) which were reworked into the Delaware Basin from the adjacent Northwest Shelf. In thin section one sees compaction of the sandstones, some quartz overgrowth cement (minor), clacite cementation (very 


\section{$0.3 \quad 103.1$}

56.3

$0.3 \quad 103.4$

56.0

$\begin{array}{lll}0.4 & 103.8 & 55.6 \\ 0.4 & 104.2 & 55.2 \\ 1.0 & 105.2 & 54.2 \\ 0.4 & 105.6 & 53.8 \\ 0.7 & 106.3 & 53.1\end{array}$

extensive), numerous fine-grained carbonate clasts mixed with we11-rounded and we11-sorted quartz and feldspar grains. The detraital carbonate grains show intense pressure solution, especially where they are in direct contact with the less soluble quartz or feldspar grains.

An enigmatic Brushy Canyon-Cherry Canyon contact is exposed in this outcrop. The contact strikes $\mathrm{N} 30^{\circ} \mathrm{E}$ and dips at about 17 degrees to the southeast. This relationship has been variously explained as a fault contact, an erosion surface, or a large and coherent slide mass. Note the abundant high-angle faults in the outcrops in this area. They generally have only minor offsets (although a few have $100 \mathrm{ft}$ or greater throw) and are part of the Tertiary block fault system which marks the western boundary of the Guadalupe and Delaware Mountains.

OPTIONAL STOP. Shales, siltstones, and sandstones of the Cherry Canyon Formation are seen here. They show a number of interesting sedimentary features including a large channel, graded beds, flame structures, ripple marks, slump folds, and abundant horizontal lamination. Again, as for the Brushy Canyon section, evidence is present for the involvement of both traction and suspension processes in the deposition of these units.

The Cherry Canyon Formation is about 1,000 ft thick in this area and thickens to about $1,300 \mathrm{ft}$ in the subsurface sections measured to the east. Organic carbon-rich shales and limestones within the Cherry Canyon Formation may have acted as source rocks for a significant part of the oil in the Permian strata of the Delaware Basin.

Road cut in Tertiary-Quaternary alluvium.

Thin-bedded Cherry Canyon Formation exposed on right.

Rest area on left.

Fault zone. Graded carbonate beds are present in the Cherry Canyon Formation at this locality.

Crest of Guadalupe Pass (elevation 5,695 ft). Pine Springs Canyon is visible to the left; the Pine Springs Camp and gas station are just ahead. The cliffs to the left (10:00 $\circ^{\prime}$ clock) are composed of Guadalupian basinal facies from the base to the middle of the slope; that is overlain by a thick zone of fore-reef rubble which, in turn, is capped by a thin zone of preserved reef limestone. The Getaway Limestone is visible in hills on the right and overlying sediments of the Cherry Canyon Formation are present in the slopes ahead and to the left. The greenish outcrops in these slopes (for example on Nipple Hill, directly ahead) are intercalated bentonite beds (volcanic ash) and shales in the Manzanita Limestone Member of the Cherry Canyon 


\begin{tabular}{|c|c|c|c|}
\hline 1.3 & 107.6 & 51.8 & $\begin{array}{l}\text { Guadalupe Mountains National Park headquarters (Frijole } \\
\text { Station) on the left. This park is one of the newest } \\
\text { in the National Park system, having opened in } 1972 . \\
\text { In } 1978 \text { approximately } 60 \text { percent of the park was } \\
\text { designated as wilderness area, precluding any large- } \\
\text { scale development. The establishment of the park } \\
\text { resulted, in large part, from the concern and gener- } \\
\text { osity of Wallace Pratt, one of the first geologists } \\
\text { of the original Humble Oil and Refining Co., who lived } \\
\text { for many years in the McKittrick Canyon area. Pratt's } \\
\text { gift to the government of more than 5,000 acres was } \\
\text { the first concrete step toward the formation of this } \\
\text { park. }\end{array}$ \\
\hline 1.3 & 108.9 & $50 \cdot 5$ & $\begin{array}{l}\text { Exposures of Cherry Canyon Formation sandstone; this } \\
\text { section is just below the South Wells Limestone } \\
\text { Member. }\end{array}$ \\
\hline 0.8 & 109.7 & 49.7 & Quaternary fanglomerates in road cuts. \\
\hline 0.5 & 110.2 & 49.2 & $\begin{array}{l}\text { Road cuts in Cherry Canyon Formation. The South Wells } \\
\text { Limestone Member is present at the top of this ex- } \\
\text { posure and consists of thin-bedded sandstone and } \\
\text { thin, lenticular, brachiopod-bearing limestones. } \\
\text { The limestone beds range in thickness from a few } \\
\text { inches to a few feet and are generally micritic. The } \\
\text { intercalation of thin limestones and sandstones tends } \\
\text { to lower the erosional resistance of this unit and } \\
\text { therefore it does not form a prominent scarp. This } \\
\text { is not true of the other limestone members of the } \\
\text { Cherry Canyon Formation, however. }\end{array}$ \\
\hline 1.0 & 111.2 & 48.2 & $\begin{array}{l}\text { We are descending off the Rader Ridge. The ridge is } \\
\text { capped by the Rader Limestone Member of the Bell } \\
\text { Canyon Formation (the third named member up from } \\
\text { the base of the formation). We are now, once again, } \\
\text { passing through the upper part of the Cherry Canyon } \\
\text { Formation. }\end{array}$ \\
\hline 0.2 & 111.4 & 48.0 & $\begin{array}{l}\text { Nickle Creek Exxon Station on the left. The greenish, } \\
\text { bentonitic beds of the Manzanita Limestone Member } \\
\text { are visible at 11:00 } 0^{\prime} \text { clock, about half way up the } \\
\text { hill. }\end{array}$ \\
\hline 0.8 & 112.2 & 47.2 & $\begin{array}{l}\text { Road cut in Manzanita Limestone. The Manzanita is } \\
\text { between } 100 \text { and } 150 \mathrm{ft} \text { thick in this area. }\end{array}$ \\
\hline 0.3 & 112.5 & 46.9 & $\begin{array}{l}\text { STOP II-4. This section shows a major submarine slide } \\
\text { deposit at the base of the Rader Member of the Bell } \\
\text { Canyon Formation. The southwestern end of the out- } \\
\text { crop consists of laminated sandstones and siltstones }\end{array}$ \\
\hline
\end{tabular}

Formation. U.S. Highway $62-180$ is still cut through the extensively faulted zone on the western side of the Guadalupe and Delaware Mountains. Numerous small faults are present here, as they have been in most of the outcrops we have passed in the last 10 to 15 miles. The ruins of a way-station of short-lived Butterfield Overland Stage route are located behind the Texas Highway Department garage on the left. As with other parts of this route, the station was abandoned in 1859.

Station) on the left. This park is one of the newest in the National Park system, having opened in 1972. scale development. The establishment of the park osity of Wallace Pratt, one of the first geologists of the original Humble Oil and Refining Co., who lived for many years in the McKittrick Canyon area. Pratt's gift to the government of more than 5,000 acres was the first concrete step toward the formation of this park. imestone Quaternary fanglomerates in road cuts. ad cuts in Cherry Canyon Formation. The South Well posure and consists of thin-bedded sandstone and thin, lenticular, brachiopod-bearing limestones. The limestone beds range in thickness from a few inches to a few feet and are generally micritic. The intercalation of thin limestones and sandstones tends to lower the erosional resistance of this unit and therefore it does not form a prominent scarp. This is not true of the other limestone members of the are descending off the Rader Ridge. The ridge is capped by the Rader Limestone Member of the Bell Canyon Formation (the third named member up from ckle Creek Exxon Station on the left. The greenish, are visible at 11:00 $0^{\prime}$ clock, about half way up the hill. between 100 and $150 \mathrm{ft}$ thick in this area. deposit at the base of the Rader Member of the Bell crop consists of laminated sandstones and siltstones In 1978 approximately 60 percent of the park was 
of an unnamed member of the Bell Canyon Formation. At the top of this sandstone is a conglomerate zone with limestone blocks set in a sandstone matrix. The limestone clasts are very poorly sorted and range from pea-sized pebbles to car-sized boulders (fig. 12). The clasts are non-dolomitic, generally lightcolored limestones derived from the Capitan reef and upper fore-reef environments. Above this zone of bouldery rubble is a thick, graded bed of similar, but finer grained, carbonate clasts with carbonate matrix and cement. This, in turn, is capped by a series of thin-bedded, fine-grained, dark-colored limestones which are typical of the basinal

limestone members of the Bell Canyon Formation.

This slide deposit is one of several which have found in the Delaware Basin. Three superimposed slides within the Rader Member make up the hummocky Rader Ridge in this area. Other slides are locally present in the Manzanita Member of the Cherry Canyon Formation and at the top of the Lamar Member of the Be11 Canyon Formation (Newe11 and others, 1953, p. 6977). These are, however, exceptional and localized events which move reef- and slope-derived material far beyond the range of the normal fore-reef rubble fans. For example, the outcrop we are at represents a slender, perhaps channelized, tongue of rubble which extends off a broader slide. This tongue of transported debris extends nearly five miles into the basin from the reef crest. This deposit has been shown to thin rapidly from reef to basin (Newe11 and others, 1953). It is nearly $100 \mathrm{ft}$ thick at the base of the steep fore-reef slope but has thinned to less than $10 \mathrm{ft}$ at this locality.

The mechanism of transport of the limestone clasts probably is largely as a submarine slide or debris flow. The volume of material involved is comparable to that of large, documented, subaerial landslides (Newe11 and others, 1953, p. 77). As with subaerial landslides, there is remarkably little disturbance of underlying soft sediment substrates. The incorporation of sandstone matrix with limestone boulders, and the channelized or abruptly terminated margins of the slides indicates that there was some erosion and inclusion of the underlying Bell Canyon sandstone in the slide. There may also have been some subsidence or foundering of the large, heavy, limestone blocks into the underlying sands.

The event which triggered the slide also, apparently, led to the generation of a turbidity current which deposited the thick graded bed which overlies the slide. This association appears to be a common one and has even been observed in modern submarine slides.

The Rader Limestone Member has a total thickness 
$0.1 \quad 112.6$

0.5113 .1

46.8

46.3

$\begin{array}{lll}0.7 & 113.8 & 45.6 \\ 0.1 & 113.9 & 45.5 \\ 1.0 & 114.9 & 44.5\end{array}$

of about $15 \mathrm{ft}$ in this area, and about $10 \mathrm{ft}$ is exposed at this outcrop. The unit thickens to a maximum of about $120 \mathrm{ft}$ within about 3 miles as one approaches the basin margin (to the northwest). Road cuts in Rader Limestone.

STOP II-5. We11-sorted, subarkosic sandstones of the Bell Canyon Formation showing remarkable uniformity of bedding and horizontal lamination. The Bell Canyon Formation is about $700 \mathrm{ft}$ thick at its type locality, but has been reported to be as thick as $860 \mathrm{ft}$ in subsurface sections (Hayes, 1964, p. 14). As rjith the other basinal sandstone units of the Delaware Mountain Group, the depositional mechanisms of the Bell Canyon sandstones have been extensively debated. The abundance of horizontal lamination and the apparently eluxinic conditions in the basin center lend credence to the idea of density overflows and suspension deposition of much of the sand and silt. On the other hand, the presence of numerous subparallel erosional channels, most of thern oriented from northeast to southwest, indicates that seafloor erosion, transportation, and deposition by long-lived density underflows, turbidity currents, or grain flows were also important.

More than 100 oil and gas fields have been discovered in the Bell Canyon Formation as of 1979 (table 3). These "are stratigraphic-hydrodynamic traps which occur where sandstone-filled channels are incised into less permeable interchannel sandstone" (Williamson, 1979, p. 39). These channels are as much as 5 miles wide, $100 \mathrm{ft}$ deep, and 50 miles long and the shape and orientation of these channels clearly controls the size, trend, and productivity of oil and gas fields.

Entrance to McKittrick Canyon day-use area of the Guadalupe Mountains National Park on the left. See McKittrick Canyon supplementary roadlog.

The escarpment ahead is formed by the Lamar Limestone Member of the Bell Canyon Formation.

S'TOP II-6. This section exposes basinal, black, laminated limestones and shales of the Lamar Limestone Member (fig. 13). Some features indicative of turbidite deposition of platforin-derived, finegrained carbonate sediment can also be seen. The Lamar is largely unfossiliferous at this locality. It becomes darker and more organic carbon-rich toward the basin center; indeed, all benthic organisms are absent from these basin-center sediments. Conversely, the unit becomes lighter colored and more fossiliferous toward the basin margin. It seems, therefore, that euxinic conditions were largely restricted to the deepest parts of the Delaware Basin (Babcock, L. C., 1977).

In this area, relatively near the basin margin, a 


\section{$1.2 \quad 116.1 \quad 43.0$}

$7.9 \quad 124.0 \quad 35.4$

$1.4 \quad 125.4 \quad 34.0$

$0.1 \quad 125.5 \quad 33.9$

$1.6 \quad 127.1 \quad 32.3$

$0.5 \quad 127.6 \quad 31.8$ moderately diverse fauna which includes burrowing pelecypods, siliceous sponges, holothurians, and conodonts, is evidence that conditions here were not uniformly anaerobic (Babcock, L. C., 1977). Yet the evaporite crystal casts found on many bedding surfaces, the organic carbon-rich sediment, and the widespread preservation of very fine-scale lamination a11 indicate that largely, euxinic, evaporitic bottom waters occupied this region during much of Lamar time. Presumably, the dominantly anocix conditions were periodically relieved by input of turbidity currents bringing sediment-laden, oxygenated waters downslope into the basin. These events were probably accompanied by short-lived but widespread colonization of the basin floor by benthic organisms.

The lateral thickness variations of the Lamar follow a similar pattern of basin-margin to basincenter change. The Lanar thins from $300 \mathrm{ft}$ along the Capitan slope, to approximately $20-30 \mathrm{ft}$ in this area, to as little as $6 \mathrm{ft}$ in outcrops about 17 miles from the basin edge, and eventually to only a few feet of silty shale in subsurface sections near the basin center (Tyrre11, 1969; Babcock, L. C., 1977).

The Lamar is the youngest limestone unit in the Guadalupian part of the Delaware Basin. As such, it is a lateral facies equivalent of the uppermost part of the Capitan Limestone on the shelf edge, and the Tansill Formation in back-reef, shelf-interior areas. Roadside rest area on right. Excellent exposures of reef and fore-reef deposits can be seen to the southwest. The exposed part of the reef becomes progressively older toward the south. The crest of the reef at the southern end of its outcrop (near Guadalupe Peak) is approximately 1,000 ft lower stratigraphically than the reef exposed at Walnut Canyon, about 25 miles to the north of this location. This implies that the face of the reef has been eroded back by at least $1 / 2$ mile in the southern Guadalupe Mountains region.

Straight ahead lies the solution escarpment of the Castile evaporites. We are driving on a surface of Quaternary gravels which lie on the basal limestone and shale unit of the Castile and on the Lamar Limestone.

Junction with Texas Ranch Road 652 on the right. Continue straight ahead.

Texas-New Mexico state line. Welcome to New Mexico. Notice the difference in vegetation on the gravel surface on which we are now driving versus that on the hills of Castile gypsum and anhydrite directly ahead.

STOP II-7. Excellent exposures of the Castile Formation in deep roadcuts. This unit is the oldest post-Guadalupian sediment in the region and conformably 
overlies the Guadalupian Bell Canyon Formation. The Castile is entirely confined to the Delaware Basin and does not extend onto the adjacent shelf areas. It has a thin, basal limestone and shale zone which may be a lateral facies equivalent of the very youngest part of the Capitan and Tansill Formations. The bulk of the Castile, however, consists of a thick section of laminated anhydrite with intervals of laminated halite. The Castile Formation has been reported to reach a maximum thickness of 1,550 to $2,000 \mathrm{ft}$ in subsurface sections in the northeastern part of the Delaware Basin (King, 1948, p. 89).

The Castile grades conformably upward into the Ochoan Salado Formation; the Salado contains laminated halite, anhydrite, sylvite, polyhalite, and even more soluble evaporite minerals. The extreme solubility of its components means that the Salado does not generally appear in outcrop. Indeed in this area, much (or a11) of the Salado may have been removed by erosion. The Salado does, however, form a wedge of sediment which thickens toward the the northeast to a maximum of greater than $2,000 \mathrm{ft}$ (Anderson and others, 1972, p. 82). In the northeastern part of the Delaware Basin, the Salado is extensively mined for potash minerals. Unlike the Castile, the Salado Formation extends beyond the borders of the Delaware Basin onto the surrounding shelf areas where it generally lies directly on Guadalupian carbonate rocks. The Salado, in turn, is unconformably overlain by the dolomitic Upper Permian Rustler Formation, the Dewey Lake Redbeds, and younger units. The pre-Rustler unconformity shows extensive Permian tilting and erosion for, in places (particularly the southwestern part of the region), it has completely removed the Salado, allowing the Rustler to lie directly on the Castile Formation or Guadalupian carbonate rocks.

The onset of Castile evaporite deposition coincided closely with the termination of reef growth around the Delaware Basin margin. It is not entirely clear whether this is a causal or coincidental relationship. Eustatic sea level drop, tectonic movements, reef growth, or other factors could have increased the restriction of influx of normal marine water into this already partially barred basin. This, coupled with the extreme aridity and high evaporation rates in the area, may have led to drastic increases in the salinity of basin water, with the associated killing of the salinity-sensitive reef organisms and the eventual start of evaporite deposition. It must be emphasized, however, that although the changes in depositional patterns at the GuadalupianOchoan transition were dramatic, the causes of these changes may have been considerably more subtle. 
Strongly evaporitic conditions existed throughout Guadalupian time as, apparently did hypersaline stagnant bottom waters in the basin. Marine influx from the south was certainly present during Guadalupian time to maintain normal marine conditions in the surface waters of the Delaware Basin. This influx must have continued through much of Ochoan time, if in a somewhat more restricted form, to supply the salts of the Castile and Salado Formations. Thus, it appears most likely that it was a gradual change in marine water supply versus evaporative water removal which led to the abrupt shift from carbonate to evaporite sedimentation, presumably when a critical salinity level was reached. This gradual (but not perfectly continuous) salinity transition apparently continued through Ochoan time, leading to deposition of anhydrite, then halite and sylvite, and eventually the true bittern salts found in the northeastern Delaware Basin.

The Castile Formation, then, represents an evaporite filling of the approximately $1,800 \mathrm{ft}$ deep basin left at the end of Guadalupian time. Although there may have been some drop in basinal water levels, the Castile clearly was deposited in deep water as indicated by the complete absence of shallow-water sedimentary structures and the presence of finescale lamination. The laminae consist of regular (although variable thickness) alternations of white anhydrite laminae and darker laminae containing a mixture of organic matter and calcite (fig. 14). The anhydrite-calcite couplets average 1-2 $\mathrm{mm}$ in thickness throughout the Castile Formation (Anderson and others, 1972 p. 73). On outcrop, the anhydrite may have been altered to gypsum (this locality has both gypsum and anhydrite exposed according to $S$. D. Kerr in Dunham, 1972). The laminations have remarkable lateral continuity, as one might expect for deeperwater evaporites, and individual laminae have been traced for more than 70 miles (Anderson and others, 1972). Contortion and deformation structures (fig. 15) are post-depositional and presumably represent volume changes due to hydration and/or dehydration reactions.

The laminations of the Castile Formation (as well as those in the uppermost Bell Canyon and Salado Formations) have been interpreted as annual varves (Udden, 1924; Anderson and others, 1972). The calcite and organic-matter layers represent periodic (annual?) freshening of the water and the development of plankton blooms. The anhydrite layers represent restricted, more evaporitic conditions. Approximately 260,000 such cycles have been counted in the uppermost Bell Canyon-Castile-Salado sequence. This implies extremely rapid deposition of thousands 
of feet of evaporites in the Delaware Basin, a common situation with major evaporite deposits.

The evaporite filling of the Delaware Basin is largely responsible for the spectacular exposures of the Guadalupian facies which we are seeing on this trip. The complete plugging of the "hole" left at the close of Capitan reef growth and the subsequent, Tertiary, removal of that plug has left us with resurrected Guadalupian topography and facies relations in this area.

The Castile and Salado evaporites may also have had a major impact on the oil and gas distribution in the Permian Basin. The rapid burial of basinal source rocks to depths sufficient for oil and (or) gas generation is one probable effect. It is quite possible that compactional geopressuring of the basinal sediments resulted from the rapid deposition. This may have eventually aided the early migration of hydrocarbons from the hasin, before deep burial and destruction of porosity in potential shelf reservoirs. Overpressuring and early oil migration may have been significant factors in the excellent hydrocarbon productivity of the Permian Basin region. The early oil movement may also explain why primary porosity and early diagenetic porosity modifications, rather than later diagenetic porosity types, are so important in many Permian Basin reservoirs. Finally, the extensive blanketing of both shelf and basin by an impermeable cover of evaporites clearyly provided an outstanding seal for the entire region.

$\begin{array}{lll}1.9 & 129.5 & 29.9 \\ 1.1 & 130.6 & 28.8\end{array}$

Note hummocky, solution-generated topography on top of the Castile evaporite. These are the Yeso Hills. White's City visible directly ahead in the distance. The valley to the left is developed on the uppermost Bell Canyon strata (Lamar and post-Lamar beds). The Capitan reef escarpment can be seen plunging to the north beneath Ochoan and younger sediments as a consequence of structural tilting. To the south, the reef rises higher and higher on the skyline to the point where it has been removed by erosion.

The buildings at the entrance to Carlsbad Caverns can be seen on the ridge top at about 11:00 o'clock.

$1.2131 .8 \quad 27.6$

$1.4 \quad 133.2$

26.2

$1.2134 .4 \quad 25.0$
Zone of Quaternary rubble probably dervied from dissolution of upper Castile or Salado evaporites. Several thin, weathered, basaltic igneous dikes cut the evaporite section in this area.

Roadside rest area on left.

At a point approximately $300 \mathrm{ft}$ east of the highway, loose boulders of Lower Cretaceous (Commanchean) limestone have been described by $\mathrm{N}$. B. Lang who interpreted them as fragments of widespread Cretaceous cover down-dropped and preserved in solution pipes cut into the Ochoan evaporites. Entrance road to Slaughter and Rattlesnake Canyons and 


\section{$0.3 \quad 136.2 \quad 23.2$ \\ $1.8 \quad 138.0$ \\ 21.4 \\ 3.3141 .3 \\ 18.1}

$\begin{array}{lll}5.0 & 146.3 & 13.1 \\ 0.2 & 146.5 & 12.9\end{array}$

$\begin{array}{rrr}1.5 & 148.0 & 11.4 \\ 0.7 & 148.7 & 10.7 \\ 2.6 & 151.3 & 8.1\end{array}$

$1.8 \quad 154.1$

$1.3 \quad 155.4$

$0.4 \quad 155.8$
New Cave on left. Both Rattlesnake and Slaughter Canyon have excellent exposures of the late Guadalupian fore-reef, reef, and back-reef facies (see Pray and Esteban, 1977). Continue straight ahead.

Highway is still on Castile Formation. The mouth of Slaughter Canyon is visible at about 8:00 o'clock; the mouth of Rattlesnake Canyon can be seen at about 9:00 o'clock. The northwest-southeast trending Huapache Monocline crosses the Capitan reef front between these two canyons.

Quaternary gravel in road cut.

Beautiful downtown White's City. Junction with New Mexico Highway 7 to Walnut Canyon and Carlsbad Caverns on left. See separate "Walnut Canyon" supplementary road $\mathrm{log}$. Continue on U.S. Highway 62180 to Carlsbad, New Mexico.

Bridge over Jurnigan Draw. Rustler Formation red beds can be seen in middle distance on right.

Junction with New Mexico Highway 396 on right. The Black River oil field is located to the right; the field produces $42^{\circ}$ API gravity oil from sandstones and siltstones just beneath the Lamar Limestone member of the Bell Canyon Formation at about 1,950 ft depth. The hills to the left are composed of Capitan reef limestone.

Road cut in Rustler Formation.

Road cut in Rustler Formation.

Junction with Dark Canyon Road on left near old Frontier Trading Post and Museum. The hills to the west are composed of Tansill Formation nearback-reef limestones and dolomites. The one well Dark Canyon oil field lies about $1 / 2$ mile to the west. Completed in 1952, the field produced from an $11 \mathrm{ft}$ pay zone in Delaware Mountain sandstone at $1,876 \mathrm{ft}$. The well continued to produce for many years at 10 to 12 BOPD. Continue straight ahead for Carlsbad. See supplementary "Dark Canyon-Sitting Bull Falls-Rocky Arroyo" roadlog for route to left.

$1.0 \quad 152.3 \quad 7.1$

In the foreground to the left are the Frontier Hills composed of Ochoan Rustler Formation sediments which dip southeastward into the Delaware Basin. The Rustler Formation in this area consists of dolomite, red beds, fine-grained sandstones, and minor gypsum. The Rustler overlies the Salado Formation in the Delaware Basin but lies directly on the Capitan Limestone in the ridge west of the Frontier Hills.

5.3 Carlsbad city limit.

4.0 Quaternary caliche exposed in pits on right.
3.6 Caverns City Air Terminal (Carlsbad municipal airport) entrance on left. The Hackberry Hills to the west are composed mainly of Tansill dolomites and upper Yates dolomites and sandstone---both back-reef facies equivalents of the uppper part of the Capitan reef. 
2.5158 .3

$1 \cdot 1$

0.1158 .4

1.0

$0.3 \quad 158.7$

0.7

The reef itself is completely buried beneath younger sediments in this area. The back-reef equivalents are exposed only because of the gentle (approximately 5 degree) eastward dip of the Guadalupian strata in this area.

$0.7 \quad 159.4 \quad 0.0$ Rodeway Inn on right. Holiday Inn on right. Ocotillo Hills located to the northeast. Junction with U.S. Highway 285 to Pecos, Texas on the right. Road $\log$ ends here. 


\author{
Mileage Cum. \\ Mileage \\ $0.0 \quad 0.0$ Junction of U.S. Highway 62-180 with paved road to McKittrick \\ Canyon day-use area (mileage 113.8 on E1 Paso-Carlsbad \\ road $\log )$. Take McKittrick Canyon road. Most of this new \\ road is cut in the McCombs to Rader interval of the Bell \\ Canyon Fm. The cliffs on the right (north) are capped by \\ the Lamar Limestone Member. \\ $0.4 \quad 0.4$ The view ahead is directly into the steeply dipping Capitan \\ fore-reef talus deposits. \\ 1.6 2.0 The view to the northwest shows the exhumed Delaware Basin \\ margin, largely stripped of its evaporite filling. The \\ reef-massif is characterized by an apparent lack of bedding \\ and a strongly developed vertical joint pattern which trends \\ parallel to the reef front. These were penecontemporaneous \\ growth faults probably produced by compaction of the 1,500- \\ $2,000 \mathrm{ft}$ of largely unconsolidated reef talus over which \\ the reef was prograding. The penecontemporaneous formation (and \\ filling) of the joints is shown by the fact that locally one \\ finds specialized Permian faunas lining fracture walls. The \\ joints are also filled with submarine calcite cement \\ crusts, Permian siltstones, soil crusts, and other \\ materials. \\ Steeply dipping fore-reef deposits are visible down- \\ slope from the reef and these form a smooth transition \\ to the nearly flat-lying toe-of-slope and basinal deposits \\ seen at the base of the escarpment. \\ 2.04 .0 Outcrops of massive, fine-grained, toe-of-slope deposits of the \\ Lamar Limestone on the left. Large-scale lenticular bedding is \\ clearly visible. Although largely composed of micritic \\ limestone, these deposits contain a significant reefal fauna. \\ Thus, they were originally interpreted as bioherms formed in \\ deep water (depths probably in excess of $1,600 \mathrm{ft}$ ). However, \\ recent studies of the Florida Straits and similar areas \\ have shown that such deposits can be produced by a combina- \\ tion of down-slope reworking of reefal debris, along- \\ slope transport and deposition by contour currents, and \\ submarine cementation. Thus, these lenticular deposits \\ may be analogous to the "lithoherms" of the Florida-Bahamas \\ region. \\ 0.3 4.3 McKittrick Canyon parking area. STOP II-1. We will walk up \\ the stream bed for a short distance (about $1 / 2 \mathrm{mi}$ ) and then \\ climb up the spur on the south side of McKittrick Canyon, \\ an approximately $1,000 \mathrm{ft}$ climb. Please wear sturdy hiking \\ boots as we will be crossing sharp rock- and cactus-covered \\ terraine. Also remember that we are now in the Guadalupe \\ Mountains National Park and rock hammering or collecting is \\ not allowed without a permit. \\ Finally, the difficulty of the terraine, the size of \\ the group, and the lack of a clearly marked trail make it \\ imperative that we all stay together as a group. PLEASE, \\ DO NOT WANDER OFF ON YOUR OWN OR IN SMALL SUBGROUPS. We
}


will keep the pace slow enough so that we can all remain together.

The purpose of this stop is to examine the toe-of-slope and fore-reef facies transitions of the Capitan limestone and its equivalents. Although it would be pleasanter if this facies change could be seen without resorting to a strenuous climb, this is not possible. It must be remembered that the facies transition is one which took place on a 30 degree slope over a vertical distance of more than $1,000 \mathrm{ft}$; thus, the vertical component is an important one. Also, these facies are exposed only in areas south of White's City and are easily accessible only in Rattlesnake, Slaughter, and McKittrick Canyons. A11 three areas require extensive climbing and McKittrick provides the best visual continuity of lateral facies.

Our upward climb will take us across a number of different Bell Canyon units (fig. 16). We start at the level of the Rader Limestone, and cross several unnamed sandstone units, and the McCombs Limestone before reaching the Lamar Limestone. We will then descend along the Lamar dip-slope and view the lateral changes within a single unit.

Because there is no well-marked path, it is not possible to provide a detailed description of this tour. However, it is possible to provide a general picture of the salient features of the route. We start in the thin-bedded, darkcolored, micritic limestones of the Rader. Bryozoans and brachiopods are the most commonly seen megafossils. Chert nodules and silicified (originally calcitic) organisms abound ( $f i g .17$ ) with the silica having been derived from siliceous sponges (fig. 18) and radiolarians which lived or accumulated in the down-slope area. The only other macroscopic diagenetic feature visible is compactional deformation around fossils, concretions, nodules, or all ochtonous blocks of reefal debris (fig. 19). Channels filled with cross-bedded reef- and slope-derived debris and large blocks of reef limestone also can be seen locally in these beds.

As we move upslope, we will see thick packages of finegrained, well-sorted sandstone and siltstone interbedded with the limestone members. The sandstones are compositionally identical to the thin back-reef sandstone and siltstone units we will see at other localities. The up-slope interfingering of discrete, basinward-dipping sandstone and carbonate units indicates that both were derived from the shelf. The sandstones also show extensive evidence of down-slope current transport including cross-bedding, channels, ripple marks, and other features. The farther upslope we move the more dominant the carbonate units become; at the same time, the sandstones become thinner and eventually pinch out entirely. The carbonate units become progressively more massive and coarser-grained in an up-slope direction (fig. 20); the carbonate grains also tend to be more clearly recognizable as reef- and slope-derived 
skeletal fragments. The fact that this is primarily reef rubble is clear from the abundance of original framework producing organisms, such as calcareous sponges and bryozoans, as well as the organic encrustation and submarine cementation of many of the clasts. In local areas, massive, sorted, and channelized grain flow and turbidity current deposits of carbonate material are visible. In most areas, however, these units have little obvious internal structure.

The diagenesis of these units is complex. Many of the clasts of calcareous debris underwent submarine cementation in their original environment of formation. Subsequent alteration in the down-slope depositional environment included (at least locally) partial, late-diagenetic dolomitization (fig. 21), leaching of aragonitic grains, and medium-crystalline calcite cementation. Compaction is not extensive in these units, but grain fracturing is common (fig. 22), and porosity is generally quite low.

As we approach the crest of the hill, apparently in situ and extensively encrusted material becomes dominant. Some of this may represent blocks of debris too large to recognize, but most of it presumably indicates in situ lithification and encrustation of material at the base of the active reef-forming area by organic and inorganic agencies. Reef growth, algal encrustation and inorganic (submarine) cementation extends into water depths of several hundred feet in many modern tropical reef areas. Similar patterns are to be expected in the Permian. Thus, although the zone of major faunal growth and diversity lay upslope from the highest point we will ascend to, some in situ growth of reef organisms, algal encrustations, and submarine cementation presumably extended downslope into the areas we are crossing.

The view from the small peak we have climbed is one of the finest in the Guadalupe Mountains from a geological perspective. This vantage point allows us to look directly at the north wall of Mckittrick Canyon (fig. 16) and largely eliminates the problem of apparent dips which complicated our earlier panoramas. The vast bulk of the sediments which make up the lower $3 / 4 \mathrm{th}$ of the north wall of McKittrick consist of Capitan fore-reef debris (fig. 16). In many areas, particularly near the mouth of the canyon, the steep, dip (nearly 30 degrees) of these rubble beds is apparent. The gradual flattening of those dips to the near-horizontality of the basinal Bell Canyon sediments is equally apparent in that area. Indeed, the uppermost basinal limestone (the Lamar) can be traced as a virtually continuous bed from basin to shelf (fig. 16) as it rises more than 1,700 ft. The same transition can be viewed in the shelf-edge escarpment visible to the north and northeast.

Above the bedded Capitan talus lies a massive, nearly completely unbedded zone of the Capitan reef-massif (fig. 16). The massive character is a consequence of both the original skeletal framework with its encrusted 
(boundstone) fabric, and the massive, penecontemporaneous cementation which completely pervaded the reef and obliterated virtually all porosity. Although particularly well developed at its eastern end, the reef massif can be traced continuously toward the west. In this direction, the reef passes lower and lower in the section but continues to overlie thick, reef-talus deposits. Thus, from this vantage point we can see that the Capitan reef built upward nearly $1,000 \mathrm{ft}$ during the time in which it prograded out over its own debris; the debris, furthermore, filled in a basin of between 1,000 and 2,000 ft water depth.

The ratio of reef rubble to in situ reef is extremely high, a fact noted by many workers. As pointed out by Dunham (1972), however, this is not a surprising situation. In modern reef-forming areas, the zone of significant reef growth is narrow, both laterally and vertically. The active growth zone is but a thin veneer on the upper and frontal edge of the reef platform. Much of this in situ material is eventually broken up by storms and reworked reworked down the fore-reef slope. New organisms grow in the reef crest area only to be reduced to rubble as we11. Vast quantities of material are formed within the reef zone but only a small fraction of this volume remains in that environment. Most is swept away into fore-reef or backreef settings. Thus, the vast amounts of rubble visible in the Capitan complex are not a valid piece of evidence to deny the existence of a true reef in this area. Rather, the rubble serves as evidence that the shelf edge was occupied by a faunally diverse assemblage of organisms with remarkably high rates of sediment production.

Above and to the west of the Capitan reef-massif, a wedge of flat lying, well bedded, back-reef sediments (Tansill and Yates Fms.) can be seen ( $\mathrm{fig} \cdot 16$ ). The wedge thickens to the west where older sediments are exposed. The sediments (mainly green algal-fusulinid grainstones) of the nearback-reef Tansill can be seen to pass into and over the Capitan reef-massif and perhaps even to spill over onto the slope in front of the reef. This may be an indication that reef growth ceased before the end of carbonate sedimentation in the area. In that case, the final phase of shelf-edge deposition would have been marked by unconsolidated skeletal sand shoals rather than a barrier reef.

The spectacular view of facies relations on the north wall of McKittrick Canyon is matched by the vista to the north and northeast. It shows present-day topography which virtually exactly matches that of late Guadalupian time. Back-reef sediments mark the upland surface of the Northwest Shelf; Capitan reef sediments, characterized by their vertical, strike-parallel jointing, delineate the upper margin or rim of the Delaware Basin; steeply dipping slope deposits compose the flanks of the escarpment; and flat-lying basinal deposits of the upper Bell Canyon Fm. compose the floor of the present basin to the east-northeast (the 
Delaware Basin). The exhumation of this Permian topography is entirely a consequence of the thick, (Castile) evaporite filling of the remnant Delaware Basin in Ochoan time, and its subsequent dissolution during the Tertiary. To the east and southeast, a series of cuesta scarps of the various limestone members of the Delaware Mountain Group are visible. These culminate in the major escarpment of the Delaware Mountains, capped by the Getaway Ls.

On our descent down the dip slope of the Lamar, on which we are now standing, we will see a progressive change in the character of the rock. We will see greater amounts of finer-grained, darker-colored, less encrusted, and less obviously detrital sediment as we move downslope. Silicification will increase we approach the base of the slope. We will eat lunch in the parking lot at the mouth of McKittrick Canyon and will then retrace our route back to U.S. Highway 62 and 180 .

4.3 8.6 Junction with U.S. Highway 62 and 180. Turn right for E1 Paso or left for Carlsbad at mileage 113.8 on the El PasoCarlsbad roadlog. 


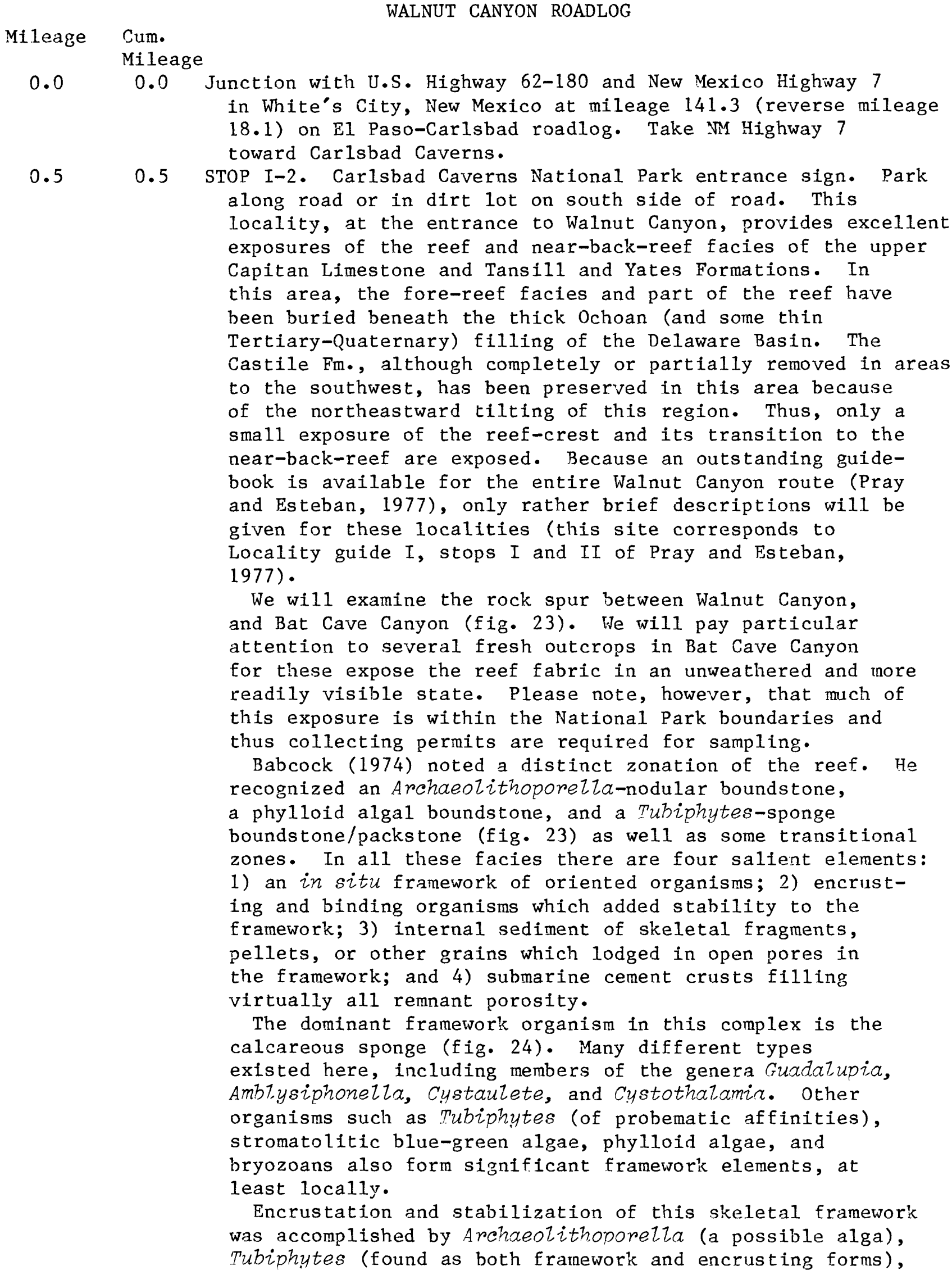


Solenopora (a probable red alga), and other, less common organisms (fig. 25). Such encrustation, seen also in modern reefs, probably contributed greatly to the strengthening of the reef framework.

Internal sediment, although not a significant factor in the lithification of the reef, did play an imporant role in infilling and occluding the primary reef porosity. Internal sediments generally are found as laminated, geopetal fabrics, sometimes with interlayered submarine cements (fig. 26). The internal sediments contain a specialized fauna including foraminifers, ostracods, echinoids, pelecypods and other organisms which presumably lived within interstices in the reef framework.

Submarine cements form a very important component of the Permian reef. Coarse fans of radial-fibrous crystals fill much of the primary porosity in the reef and make up more than half the total volume of rock in many locations. The cement fans, probably originally aragonite, are commonly interlayered with Archaeolithoporella or other encrusting organisms (fig. 25). The submarine cements are restricted to a relatively narrow zone which extends from several hundred feet down the fore-reef slope to perhaps one half mile shelfward of the reef crest. Very similar relations have been seen in modern reefs such as in Belize, Florida, the Bahamas, and Jamaica. In these areas, as in the Permian, submarine cementation, largely in the form of aragonitic and high-Mg calcite fans and crusts, are restricted to the reef face, upper fore-reef, and near-backreef zones.

After seeing these major fabric elements where they are unweathered and well exposed in Bat Cave Canyon we will cross the spur to Walnut Canyon. Examine the sediments on this traverse and try to recognize the same fabrics where they are more intensely weathered. Also examine the fracture fillings along Walnut Canyon.

We will now walk up Walnut Canyon examining the transition from reef to near-back-reef areas, eventually crossing from the south to the north side of Walnut Canyon.

On this traverse, be sure to note changes in bedding character as well as sediment composition. Also note the remarkably rapidity of the lateral lithologic changes. The most obvious change is from a boundstone fabric to one of grainstones and packstones containing ooids and skeletal grains. Cephalopods, foraminifers, pelecypods, gastropods, and most importantly, dasycladacean green algae, particularly Mizzia and Macroporella, rapidly supplant sponges and bryozoans as the major skeletal components. Bedding in these well-sorted grainstones is massive and indistinct (fig. 27) but still is far better defined than in the reef facies. Grain size ranges from coarse silt to coarse sand; sorthing moderately good to excellent. Coated grains and ooids form a significant percentage of the total sediment (figs. 27, 28 and 29).

Sediments further up-canyon (farther back-reef) show 
increasing amounts of dolomite, fenestral fabrics, coated (pisolitic) grains, algal(?) lamination, carbonate breccias, "tepee structures", and thin, clastic terrigenous sandstone-siltstone units.

The abrupt facies transition frorn reef to back-reef is similar to that seen in many modern settings. In the Florida Keys on the western side of Andros Island in the Bahamas, for examples, the change from reefal boundstones to skeletal, back-reef grainstones takes place over distances of just a few tens to hundreds of feet. The near-back-reef areas in Florida and the Bahamas generally consists of complex, small-scale mircofacies of green-algal (Halimeda) grainstones, grapestones (coated and coalesced grains), ooids, skeletal fragments, and other lithologies. In areas such as the Joulter's Cay region of the Bahamas, one can see these grainstone types closely intermingled as a series of submarine sand waves, islands, tidal channels, and beaches. Associated with these grainstones are mudstonewackestone microfacies in sheltered areas of tidal flats and back-barrier coves. The extremely varied lithologies in the Permian near-back-reef setting presumably reflects similarly complex microfacies patterns. This is also evident in the intimate mixture of diagenetic patterns in the Permian nearback-reef sediments. Submarine as well as vadose phreatic nonmarine cements are all present in local zones in this area probably as a result of local (island facies) input of nonmarine fluids.

Return to vehicle(s) and proceed up-canyon.

$0.20 .7 \quad$ Near-back-reef Mizzia-dominated grainstones on right which we have examined in our previous traverse.

$0.2 \quad 0.9$ Cross Walnut Canyon.

$0.2 \quad 1.1$ Pisolite-bearing dolomites and faulted upper Yates sandstones in roadcut.

$0.1 \quad 1.2$ Upper Yates and lower Tansill sediments in canyon walls. This locality exposes mainly pisolitic dolomites and sandstones and is an excellent area for examining tepee structures.

1.0 2.2 Exhibit area on right.

0.32 .5 Road cuts on right expose Yates Fm. dolomite and sandstone.

1.0 3.5 Parking area on left with exposures of pisolitic dolomites, tepee structures, and sandstones of Yates $\mathrm{Fm}$.

0.54 .0 Exhibit area (showing botanical diversity of the area) on the left. Canyon wall on left has exposures of Yates Fm., including the large, sand-filled cavern described by Dunham (1972, Stop II-5).

1.25 5.25 Sharp bend in road; primitive road on right can be used as parking area to view exposures of Yates Fm. just ahead.

0.055 .3 STOP III-7. Outstanding exposures of pisolitic dolomites of the upper Yates Fm. (see Pray and Esteban, 1977; Dunham, 1972). This locality illustrates numerous cycles of pisolitic sediments (termed "Walnutite cycles" by Pray and Esteban, 1977). Tepee structures (fig. 30) can be seen both in this outcrop and in the distant canyon wall to the north. The main small-scale features to be seen at this outcrop are 
the abundant pisoliths which range from B-B-size to golf bal1-size. They have concentric laminations of thin carbonate coatings around nuclei of fractured pisoliths ( $f i g, 31$ ) or, rarely, marine fossils. The pisoliths, which have been completely replaced by aphanocrystalline dolomite, occur in cyclic beds, commonly with reverse grading (fig. 31 ). In some (but not most) cases, pisoliths have intergrown or interlocking textures. There is considerable evidence to show that these pisoliths had original aragonite composition, now replaced by dolomite. They are associated with sheet cracks (broad bands of displacive, fibrous carbonate, presumably also originally aragonite but now dolomite or calcite; fig. 32). These displacive crusts are related to the origin of the tepee structures of this area for the tepees are expansion polygons formed by a volume increase of the associated sediments. This was most likely accomplished by in situ, near surface, displacive growth of aragonite and (or) evaporite minerals.

The origin of pisoliths and tepee structures in these sediments has been the subject of numerous studies and considerable controversy. Extensive discussions of these problems have been presented recently by Dunham (1972), Esteban and Pray (1977), and Pray and Esteban (1977) and so will be only briefly outlined here.

Basically there are two hypotheses: 1) the "all wet" model which proposes that the pisoliths were formed by organic (algal) or inorganic coating of grains in a shallow-water shelf setting with each grain acting as a free, clastic particle; and 2) the "caliche" hypothesis which suggests that pisoliths formed in situ as part of cyclic, reverse graded, caliche profiles which formed by alteration of carbonate sediment brought into the area by storms or other episodic processes. Advocates of either model can point to modern analogs (mainly from the Persian Gulf and Red Sea areas) with scattered, small-scale accumulations of aragonitic pisoliths in marginal marine, hypersaline settings. Yet nowhere have we discovered an analog which comes close to modeling the breadth and abundance of pisoliths that one sees in the Permian.

The differences of interpretation of these deposits, although important from the point of view of fully understanding the sediments, are not of great significance to the explorationist. There can be little argument that this facies must have stood as a paleotopographic high-point in Guadalupian time. The persistence of this facies in space and time (it is present in Seven Rivers, Yates, and Tansill rocks), its consistent geometry (an elongate facies, para1lel to the reef trend), and its equally consistent juxtaposition between open marine (grainstones with a high faunal diversity) and restricted (hypersaline mudstones and evaporites) environments indicate that the pisolite facies must have been a major hydrographic barrier. Nowhere in the world today are evaporitic mudstones and open marine, faunally diverse sediments in close proximity without 
having an intervening barrier. It seems likely that to act as such a barrier, the region would have had to be subaerially exposed (except for tidal channels) and this would favor the caliche interpretation. It is possible, however, that broad, low relief, tidal flats could also act as partial hydrographic barriers. Finally, it is possible that a combination of processes could have acted. A number of different pisoliths can be seen in the Permian strata. These range from the small, irregularly coated grains seen at Stops III-2 and I-2 (and which almost certainly formed in a marine setting) to the larger, smoother, and more extensively encrusted grains present at this locality. Thus, a number of different origins can be envisioned for the different pisolith types.

The tepee structures and sheet cracks found in association with pisolitic sediments can also be interpreted as either marine or nonmarine. Displacive aragonite crusts and tepees have been noted in submarine cemented areas within the Persian Gulf itself as well as in coastal caliches and sabkha surfaces of the surrounding, subaeria1exposed coastlines.

0.3 5.6 Exhibit area on left; the thin sandstone-siltstone unit which marks the Tansill-Yates contact is exposed on the left. The road ascends into Tansill $\mathrm{Fm}$. dolomites.

1.97 .5 Cave entrance parking lot. Stop I-3. We will do the complete walking tour of Carlsbad Caverns - the Roswell Geological Society 1964 field trip guidebook provides a trail log of the caverns (Sanchez, 1964). The cave is cut primarily in the Capitan Limestone but the entrance and all of the upper level are in the back-reef dolomites of the Tansill and Yates Fms. The lowest parts of the cave are cut in steeply-dipping fore-reef talus of the Capitan, down to a level approximately $850 \mathrm{ft}$ below the entrance. This level is presumably related to the regional groundwater discharge surface in the Pecos valley to the northeast. The history of development of the cave is extensively described by Jagnow (1979). The location and orientation of the Capitan reef and its early fracture system have controlled, to a large degree, the geometry of the local cave systems. P1iocene and Pleistocene uplift allowed percolation of phreatic groundwater through the joint system and eventual excavation of the caverns. The subsequent vadose history of the cave led to introduction (and later partial removal) of clay, silt, sand, and gypsum fills as well as calcitic speleothems. The cave is largely inactive today except for some areas in the lowest cave levels.

The outcrops at the southwest end of the parking lot provide exposures of tepee structures, sheet cracks, and pisolitic sediments of the Tansill Fm. We will probably not have time to visit these outcrops, but similar features are seen at Stop III-7.

Return down Walnut Canyon.

7.515 .0 Junction with U.S. Highway $62-180$ in White's City. End of supplementary roadlog. Turn left for E1 Paso or right for Carlsbad and rejoin main roadlog at mileage 141.2 (reverse mileage 18.1). 


\section{DARK CANYON - SITTING BULL FALLS-ROCKY ARROYO ROADLOG}

\begin{tabular}{lll} 
Mileage & \multicolumn{1}{c}{$\begin{array}{c}\text { Cum. Mileage } \\
\text { Clock- } \\
\text { wise } \\
\text { loop } \\
0.0\end{array}$} & $\begin{array}{l}\text { Counter- } \\
\text { clockwise } \\
\text { loop } \\
80.1\end{array}$ \\
0.4 & 0.4 & 79.7 \\
0.8 & 1.2 & 78.9 \\
1.5 & 2.7 & 77.4 \\
1.2 & 3.9 & 76.2 \\
& & \\
& & \\
0.15 & 4.05 & 75.05 \\
0.20 & 4.25 & 75.85
\end{tabular}

Head west on paved road to Dark Canyon at mileage 151.3 (reverse mileage 8.1) on E1 Paso-Sarlsbad roadlog.

The Hanson and Yates, King No. 1 well on the right is the entire Dark Canyon oil field. Numerous offset wells were dry holes.

Rustler Formation dolomite, sandstone, and gypsum make up the low Frontier Hills ahead.

Rustler Formation outcrops in hills on both sides of road.

Mouth of Dark Canyon. We have left the Delaware Basin and are now on the Northwest Shelf in the northeastern part of the Guadalupe Mountains. Bedded Capitan Limestone outcrops are present on both sides of the canyon.

Road intersection; take sharp right.

STOP III-1. We will examine the reef to nearback-reef transition in the uppermost part of the Capitan and lower Tansill Formations. These sediments have been mapped as reefal Capitan limestones by Motts (1962) and as backreef limestones of the Tansill Formation by Kelley (1971). Tyrrell (1969) and Toomey and Cys (1977) presented extensive evidence to show that this locality provides an exposure of the transition beds between the Capitan and Tansill carbonates. Clearly, the major part of the reef front is buried beneath basinal sediments in this area but shelfward facies are well exposed. Thus, we can examine the near-back-reef skeletal grainstones containing ooids and algally coated grains, dasycladacean green algae (particularly Mizzia and Macroporella), crinoids, belerophontid gastropods, fusulinid Foraminifera, TubiphytesArchaeolithoporeliza colonies, and sponges (fig. 33). These rocks are similar in many ways to the sediments seen at the up-canyon end of the first stop in Walnut Canyon (Stop I-2) which would inticate that we are probably at, or only a short distance shelfward, of the main reef facies. Note the consistent change in bedding character as we walk in a shelfward direction.

The abruptness of the facies transition seen here and in Walnut Canyon in the near-back reef setting is very similar to modern facies transitions in areas such as Florida or the Bahamas. There, as here, reefal debris tends to move primarily into fore-reef talus; back-reef sands 
are dominated by grains of green algal origin (Halimeda in modern sediments; Mizzia in the Permian) ooids or coated grains, and other particles of shelf origin. Submarine shoals, channels, islands, and patch reefs have local distribtuion and complex, virtually unpredictable, patterns. Such modern setting appear to be excellent analogs for these older environments.

We will not be able to stay within the Tansill Formation in our entire traverse through the Capitan-equivalent shelf strata because erosion has removed much of the far-back-reef Tansill. We will see facies equivalents in older (but still Capitan-equivalent) strata of the Yates and Seven Rivers Formations. Evidence from remaining outcrops and subsurface data indicate that similar shelfward facies transitions occurred in all three back-reef units (fig. 34). The general sequence of facies from the shelfedge landward tends to be reef; massive, skeletal (mainly green algal) grainstones; bedded and cross-bedded oolitic grainstones; dolomitized, fenestral grainstones and pisolitic mudstones; coarse, pisolitic, dolomitized grainstones with tepee structures; stromatolitic or pelloidal dolomitized mudstones; pure, calcisphere-bearing, dolomitic mudstones; with evaporite crystal casts and (or) collapse breccias; nodular gypsum or anhydrite units; and finally red siltstones.

Throughout this facies suite, thin but laterally persistent, fine-grained sandstone and siltstone beds are found. These sandstone-siltstone units, especially common in the Yates Formation, generally pinch out before reaching the reef facies; in several areas these sandstones approach within a few hundred yard of the reef. In the Yates (and the older Queen) strata, these terrigenous beds make up at least $1 / 3$ of the formation thickness, are excellent regional correlation markers, and can act as reservoir units.

Because there was extensive (2-3 mile) basinward progradation of facies during Capitan deposition, the facies previously described as being lateral equivalents can also be seen to some degree in vertical sequence, a fact which has significant influence on the early diagenetic history of much of the sediment package. Figure 34 shows the progressive basinward shift of the evaporite-carbonate transition zones in successively younger, Capitan-equivalent back-reef units. Thus, the progradation 
of shallow-water, subaerial, or restricted environments over more normal marine sections may have allowed very early input of freshwater or hypersaline brines into unconsolidated and geochemically unstable sediments. Indeed, sediments from the shelfward edge of this outcrop to the platform interior show extensive signs of vadose as well as phreatic leaching and cementation combined with virtually complete, very finely crystalline dolomite replacement. The approximate thickness of back reef units of the Artesia Group in this region are (in ascending order): Grayburg Fm., 400-500 ft; Queen Fm., 200-400 ft; Seven Rivers Fm., 450-600 ft; Yates Fm., 300-400 ft; and Tansill Fm., 100-325 ft (a11 data from Kelley, 1971). The transitions from carbonate to evaporite facies generally occur within 5 to 15 miles shelfward of the bank margin or reef throughout the history of the Artesia Group (fig. 34). Turn around and return to main road.

$\begin{array}{lll}0.20 & 4.45 & 75.65 \\ 0.55 & 5.0 & 75.1\end{array}$

Intersection with main Dark Canyon road; turn right.

STOP III-2 at junction with sma11 dirt road on left. Park and walk down road to cliff outcrop on south side of canyon. This locality (equivalent to part of Dunham's (1972) Stop I1) exposes dolomites and calcitic dolomites of the near-back-reef Tansill Formation. A wide variety of sediment types are present here, typical of the complex, sma11-scale microfacies patterns in this paleogeographic zone. We can see pisolitic mudstones, birdseye dolomites, cross-bedded green-algal grainstones, and other lithologies intimately intermingled at this site. Fusulinid Foraminifera, belerophontid gastropods, pelecypods, green algae, and probable blue-green algae are particularly abundant in these sediments.

These beds apparent1y represent a series of islands or banks (cross-bedded grainstones; fig. 35), subaerial and intertidal flats (birdseye fabrics; fig. 36), restricted or sheltered mud accumulation sites (pisolitic mudstones), and intervening tidal channels (fossiliferous packstones and grainstones). These facies patterns are quite similar to ones found in the Bahamas in regions such as Joulter's Cay, or in Trucial Coast barrier-lagoon complexes of the Persian Gulf.

Diagenetically, these Permian microfacies are equally complex. Within this outcrop one can find microscopic examples of submarine cement as well as vadose and phreatic freshwater cements 
(figs. 37 to 39). Porosities in this zone are variable but include some of the highest values found anywhere in the Guadalupian facies spectrum.

$0.3 \quad 5.3 \quad 74.8$

STOP III-3. Cross stream wash and examine two outcrops on north side of valley. The TansillYates contact is exposed in the western part of the outcrop, marked by a thin sandstone-siltstone unit. We can see algally laminated dolomites, fusulinid grainstones, pisolitic beds, probable Permian breccia pipes, as well as infiltrated, red, lateritic soils in solution enlarged fractures and voids. The pisolitic microfacies was postulated by Dunham (1972) to be one of the highest paleotopographic zones in the Capitan complex. Pisolitic "caliche" zones and solution features would thus be a probable result of even minor relative sea level drops during deposition. The red void-fillings consist of kaolinite, hematite, quartz, goethite, illite, and amorphous iron oxide, a reasonable composition for a solution residue in this area. More extensive discussion of this facies will be given at Walnut Canyon (STOP III-7).

The thin sandstone-siltstone bed at the YatesTansill contact is typical of such terrigenous units in this area. They are generally 1-8 ft thick, well-sorted, very fine sandstone or coarse siltstone, and have subarkosic or arkosic composition. Dunham (1972) showed that a progressive decrease in feldspar content of these units from shelf interior to the Capitan shelf margin is directly matched by a progressive increase in kaolinite content. Thus, these clastic terrigenous beds were probably uniformly arkosic but the near-reef sections underwent more intense post-depositional alteration.

In spite of their relative thinness, these sandstone-siltstone beds have great lateral extent, particularly parallel to the reef trend, and serve as excellent stratigraphic marker beds (DeFord and Riggs, 1941). Some low-angle channel structures and ripples can be seen, locally, in these units, but generally these sediments are horizontally laminated or structureless. They presumably represent largely wind-transported material; the horizontal lamination may have resulted from dune migration over an equlilibrium deflation surface (sabkha) or from depostion in extremely shallow, lagoonal waters.

$0.4 \quad 5.7 \quad 74.4$ Optional Stop. Pisolitic dolomites with "tepee" structures well exposed on south side of 
valley. Note laminated sandstone-siltstone unit interrupting and truncating some "tepees". The sandstone-siltstone is the uppermost part of the Yates Fm.; overlying dolomites are in the Tansill Fm.

$\begin{array}{lll}1.1 & 6.8 & 73.3 \\ 0.3 & 7.1 & 73.0 \\ 0.6 & 7.7 & 72.4 \\ 0.5 & 8.2 & 71.9 \\ 0.4 & 8.6 & 71.5 \\ 1.2 & 9.8 & 70.3 \\ 0.2 & 10.0 & 70.1\end{array}$

Yates outcrop on left contains pisolitic dolomite and sandstone.

Yates Fm.(?) pisolitic dolomite with evaporite crystal casts on right.

Yates Fm.(?) outcrop of thick-bedded, dolomitic mudstones with sparse evaporite crystal casts.

Dolomitic mudstones, peloidal grainstones, and pisolitic beds of Yates $\mathrm{Fm}$. (?) on right.

Yates Fm.(?) dolomitized peloidal grainstones on left.

Road junction; continue straight ahead to Sitting Bu11 Falls.

STOP III-4. Nalk down stream to outcrops on north side of valley. Exposure of thin-bedded, aphanocrystalline to very finely crystalline dolomitic mudstone with extensive evaporite crystal casts, pyrite nodules, and contorted, probably stromatolitic zones. These penecontemporaneously dolomitized mudstones contain pellets, some peloids, scarce encrusting foraminifers, and numerous calcispheres. These sediments, with their sparse assemblage of salinity tolerant organisms and evaporite minerals, apparently represent a shallow, hypersaline lagoon similar to those found today in many areas of the Persian Gulf.

$\begin{array}{lll}0.5 & 10.5 & 69.6 \\ 2.1 & 12.6 & 67.5 \\ 0.9 & 13.5 & 66.6 \\ 0.3 & 13.8 & 66.3 \\ 0.5 & 14.3 & 65.8\end{array}$

Road junction; bear right.

Start gravel road; continue straight ahead.

W.G. Smith ranch road on right; continue straight ahead. Road is on Seven Rivers Fm.

Road intersection on right; continue straight ahead on main road.

STOP III-5. Interbedded thin-bedded, dolomitized mudstones and red, far-back-reef siltstones of the Seven Rivers Fm. on left. The Seven Rivers is the oldest Capitan-equivalent unit in the Artesia Group. These outcrops have been mapped as basal Yates Fm. by Motts (1962) but have been considered to belong to the Seven Rivers by most other workers. Note the uniformity of the aphanocrystalline to very-finely-crystalline replacement dolomite. The environment presumably represents a shallow lagoonal or lower sabkha environment.

$0.3 \quad 14.6 \quad 65.5$

Medium-scale contortions visible in Seven Rivers Fm. These were probably caused by near-surface dissolution of interbedded gypsum and anhydrive, although the Seven Rivers consists mainly of dolomites and siltstones in this area. 


$\begin{array}{lll}0.4 & 15.0 & 65.1 \\ 0.9 & 15.9 & 64.2 \\ 1.2 & 17.1 & 63.0 \\ & & \\ 0.2 & 17.3 & 62.8 \\ 1.7 & 19.0 & 61.1\end{array}$

Road intersection on left; continue straight ahead.

Road intersection on left; bear right on main road.

Varicolored sediments on right are interbedded nassive gypsum, dolomite, and red beds of Seven Rivers $\mathrm{Fm}$.

Ranch road on left; continue straight ahead. STOP III-6. Borrow pit in gypsum of Seven Rivers Formation. Surface weathering makes viewing of gypsum outcrops a frustrating exercise; most are altered to a very great degree with a solution residue covering most fabric elements. This borrow pit exposes the freshest samples easily accessible to a field trip group. Gray-white gypsum with a nodular, enterolithic texture can be seen in isolated blocks scattered around the pit (fig. 40). This "chicken-wire" fabric may be related to a sabkha origin of the evaporite, but may also be a consequence of dehydration-rehydration reactions during burial and uplift. (a1though the unit is gypsum on outcrop, it is generally anhydrite in the subsurface). The "chicken-wire" texture, with thin clay films between gypsum nodules, also been interpreted as the product of displacive growth of subaqueous gypsum in silty-clayey sediments in a shallow-water lagoon (Sarg, in Pray and Esteban, 1977).

On the left one can see a reentrant of the Seven Rivers Embayment, an extensive planar feature developed by dissolution of the evaporites of the Seven Rivers Fm.

$\begin{array}{lll}0.3 & 19.3 & 60.8 \\ 1.2 & 20.5 & 59.6 \\ 0.8 & 21.3 & 58.8\end{array}$

Road junction to right; continue straight ahead. Road on left; continue straight ahead on main road.

Ranch house on right. Well drilled to left (Humble's Bandanna Point Unit Vo. 1 gas we11) was completed as a gas producer from Morrowan (Pennsylvanian) sandstone. It encountered the following units: San Andres Fm. (top at $750 \mathrm{ft}$ depth); Bone Spring Ls. $(2,815 \mathrm{ft})$; Wolfcamp limestone $(7,150 \mathrm{ft})$, Pennsylvanian $(7,550 \mathrm{ft})$; Mississippian (Chester) $(10,234 \mathrm{ft})$. Woodford Shale $(10,868 \mathrm{ft})$; Devonian (10,932 ft); Montoya Group (11,622 ft); Simpson Group (11,995 ft); and Ellenburger Group $(12,050 \mathrm{ft}$ ) (data from Hobbs, Roswell and West Texas Geological Societies, 1962 Field Trip Committee, 1962, p. 18).

$1.2 \quad 22.5 \quad 57.6$

Azotea Mesa on right is composed of Seven Rivers Fm. gypsum capped by a prominent dolomitic ledge. The ridge ahead in the distance consists of Queen and Grayburg beds downwarped 


$\begin{array}{lll}1.0 & 23.5 & 56.6 \\ & & \\ 2.6 & 26.1 & 54.0 \\ 3.2 & 29.3 & 50.8 \\ 1.0 & 30.3 & 49.8 \\ 0.2 & 30.5 & 49.6 \\ 0.2 & 30.7 & 49.4 \\ 0.6 & 31.3 & 48.4 \\ 0.3 & 31.6 & 48.5\end{array}$

$\begin{array}{lll}0.7 & 32.3 & 47.8\end{array}$

$0.6 \quad 329$

47.2

$0.2 \quad 33.1 \quad 47.0$

$0.3 \quad 33.4 \quad 46.7$ along the Huapache Monocline. We are now entering the main part of the Seven Rivers Embayment with the road on thin alluvium over Queen $\mathrm{Fm}$.

Intersection with New Mexico Highway 137 (a paved road from El Paso Gap to Carlsbad). Turn left toward El Paso Gap. Guadalupe Mountains ahead in distance; road traverses the Seven Rivers Embayment atop the Queen $\mathrm{Fm}$.

Junction with road to Sitting Bull Falls on right; turn right.

Queen Fm.(?) redbeds, dolomites, and evaporites on right.

Road crosses approximate Queen-Grayburg contact and passes onto Grayburg Fm.

Entering Lincoln National Forest.

Road crosses first wash and traverses Huapache monocline ahead.

Road crosses onto San Andres Limestone.

Lenticular, partly silicified, skeletal grainstones of the San Andres, probably filling channels, on right at base of slope near stream crossing. Brown, thin-bedded Cherry Canyon sandstone beds can be seen in cliffs on right. This tongue of the generally basinal Cherry Canyon Fm. extends many miles into the shelf environment in this area. The Cherry Canyon sandstone tongue is $164 \mathrm{ft}$ thick near the mouth of Sitting Bull Canyon.

A massive upper San Andres bioherm, overlain by Grayburg Fm. is visible ahead on right. Note lenticular bedding to the left of the bioherm. Junction of Sitting Bull Canyon and Last Chance Canyon (along which have been travelling). Road turns into Sitting Bull Canyon. Cliff on north side of Last Chance Canyon (on right) has exposures of lower San Andres Ls. (at very base) overlain by the thick sandstone tongue of the Cherry Canyon Fm.; the upper San Andres, bioherm-bearing limestone and Grayburg Fm. sandstones and carbonates forn the top of the section. A major angular discordance is visible between the Cherry Canyon sandstone tongue and the upper San Andres Ls. A nore subtle angular discordance is also present between the upper San Andres and the Grayburg Fm.

Excellent exposures of the lower San Andres, here extensively dolomitized, can be examined by walking up Last Chance Canyon for a few hundred feet.

The Cherry Canyon-San Andres unconformity is we11 exposed on both sides of the road.

Cherry Canyon sandstone outcrops with well 
$0.5 \quad 33.9 \quad 46.2$

$7.7 \quad 41.6$

38.5

$2.6 \quad 44.2 \quad 35.9$

$3.2 \quad 47.4$

32.7

$1.2 \quad 48.6$

31.5 developed cross-bedding are visible on right. Parking area for Sitting Bull Falls. Lunch stop at picnic benches. The entire cliff on the northwest side of the picnic area is formed of modern travertine which can be examined in numerous fallen blocks. A short walk to the active waterfall area shows travertine in the process of formation. Calcium bicarbonate and carbon dioxide saturated waters emerge from springs in the Grayburg and San Andres units a short distance (about 1 mile) upstream from the falls. Warming of the waters, combined with release of $\mathrm{CO}_{2}$ from waters plunging over the falls, induces precipitation of calcium carbonate. The withdrawal of $\mathrm{CO}_{2}$ from the water by the numerous algae and higher plants which abound at the falls also contributes to the calcite precipitation. These plants are then incorporated within the calcite, later to rot away. This accounts for the unusual fabric seen in the travertine blocks.

If you climb up or under the waterfalls, please take great care as the rocks here are very slippery. The other walls of the canyon in this area expose the Cherry Canyon sandstone tongue in their lower part, the upper San Andres Limestone in the middle part, and Grayburg $\mathrm{Fm}$. in the upper part. Note the biohermal or bank structures in the San Andres; they were probably deposited as fusulinid grainstone banks rather than as true reefs.

Return down-canyon to the E1 Paso Gap-Carlsbad road.

Junction with New Mexico Highway 137 (E1 Paso GapCarlsbad road). Note West Hess Hills at 2:00 to 3:00 o'clock and Azotea Mesa at 10:00 to 11:00 o'clock---both are composed of gypsum, red siltstones, and dolomites of the Seven Rivers Fm.

Turn left toward Carlsbad.

Unpaved Dark Canyon road on right; continue straight ahead. Road is on Queen Fm.

Road now crossing the approximate contact between the Queen and Seven Rivers Fms. The road is located almost directly on this contact for the next few miles, with Queen sediments on the left and Seven Rivers on the right.

Hills ahead and to the right are composed of Seven Rivers evaporites and red siltstones. The strongly developed vertical gullying is characteristic of the evaporite facies of the Guadalupian far-back-reef units and contrasts sharply with the horizontal bedding which dominates in areas of carbonate facies within 


$\begin{array}{lll}4.4 & 53.0 & 27.1 \\ 0.6 & 53.6 & 26.5\end{array}$

$0.1 \quad 53.7 \quad 26.4$

$2.3 \quad 56.0 \quad 24.1$

$0.1 \quad 56.1 \quad 24.0$ these same units (fig. 41). Most of these hills are capped by the resistant "Azotea Tongue" (usage of Sarg, 1976), a dolomite unit in the Seven Rivers Fm.

Road passes from Seven Rivers Embayment into Rocky Arroyo.

Road is at the level of the contact of the Queen Fm. (Shattuck Sandstone unit of Sarg, 1976) and Seven Rivers Fm. We are nearly 1 mile shelfward (northwest) of the carbonate-evaporite transition of the Seven Rivers Fm. (Sarg, 1976). This transition is remarkably abrupt (within about $500 \mathrm{ft}$ ) and remains in approximately the same location for nearly $200 \mathrm{ft}$ of section (Bates, 1942; Pray and Esteban, 1977). This Seven Rivers facies transition has been shown by Sarg (1976) to be related to a depositional ridge in the underlying Shattuck Sandstone of the Queen Fm.

The gullied hillside on the southeast side of the road has good exposures of the Seven Rivers evaporite facies (see Pray and Esteban, 1977, Stop VII). The section is dominated by bedded, nodular, mosaic gypsum with thin, pelletal or grapestone-bearing dolomites and red, gypsumcemented, sandy siltstones.

Note invigorating, heady aroma of hydrocarbons in the air; it emanates from the Indian Basin gas field about 0.3 miles ahead.

Low road cuts on right are Queen Fm. (Shattuck Sandstone). Conical hill visible to the north of the road is "The Tepee" and is capped by the resistant "Azotea Tongue" a massive dolomite of the Seven Rivers Fm. Underlying Seven Rivers evaporites, the Shattuck Sandstone and dolomites of the Queen $\mathrm{Fm}$. are also exposed.

Intersection with road on left leading to Marathon Dil Co. Indian Basin gas field and plant. Production here is from Upper Pennsylvanian and Lower Permian reservoirs. Continue on main road.

Optional stop. Excellent view of the carbonateevaporite facies transition in the Seven Rivers Fm. (see description of Stop VI in Pray and Esteban, 1977) on north wall of Rocky Arroyo. This extremely rapid transition can be seen in a narrow, nearly vertical, band in the upper half to two-thirds of the far wall of the valley. The transition is visible because of the radically different weathering patterns of the evaporite (vertical gullying) and carbonate (horizontal bedding) facies. The transition was was first described by Bates (1942) and has been recently examined by Sarg (1976). 
$\begin{array}{lll}0.7 & 56.8 & 23.3\end{array}$

$0.1 \quad 56.9$

23.2

$0.3 \quad 57.2$

22.9

0.8

58.0

22.1
Also exposed in the lower part of the cliff is the upper dolomite and the overlying Shattuck Sandstone unit of the Queen Fin. The Shattuck, generally about $90 \mathrm{ft}$ thick in this region, thickens to about $140 \mathrm{ft}$ beneath the carbonateevaporite transition and may have been partly reponsible for the generation of restricted, evaporitic conditions shelfward of this point during Seven Rivers deposition (Sarg, in Pray and Esteban, 1977).

Optional stop. Excellent exposures of Shattuck Sandstone (Queen Fm.) on the right (see Dunham, 1972, Stop I-5; Pray and Esteban, 1977, southwest end of Stop VIII). The sandstone has broad, channel-like structures with northwestsoutheast axes.

Continuation of previous outcrop. These mediumto thin-bedded dolomites, about $12 \mathrm{mi}$ shelfward of the Capitan scarp, are generally placed in the Seven Rivers Fm.; Sarg (1976), however, included them in the Queen $\mathrm{Fm}$. Some interesting collapse breccias occur in these strata, which were included by Dunham (1972) in his "calcisphere dolomite wackestone" facies. The largest breccia occurs as an isolated pocket in a thick, light-tan dolomite bed. The breccia has large, angular clasts of dolomite with corroded and altered borders (fig 42). The clasts are held in a partial matrix of microcrystalline calcite, internal sediment (green illite-kaolinite clay and quartz silt), and coarsely crystalline, blocky, late, sparry calcite cement. Considerable remnant porosity also is present in the breccia zones.

Pray and Esteban (1977) argued for a modern karstic origin for these features; Dunham (1972) postulated a Permian origin, presumably related to weathering and dissolution of evaporite minerals. Note the abundant evidence of associated evaporite (mainly gypsum) crystal casts in these dolomites.

Cyclic deposits of dolomite and red, silty shales. Generally grouped in the Seven Rivers Fm., these sediments were included in the uppermost part of the Shattuck Sandstone (Queen Fm.) by Sarg (1976) and Esteban and Pray (1977; see description for Stop VIII). These strata have been interpreted as "dolocalcrete cycles" with evidence of repeated deposition, exposure, weathering, and calichification (Pray and Esteban, 1977). Note also the abundant crystal- and nodule-casts of former gypsum (some voids now partly filled with calcite; see fig. 43).

A thick section of Seven Rivers dolomitic pack- 


\begin{tabular}{|c|c|c|c|}
\hline & & & red beds. \\
\hline 0.3 & 58.3 & 21.8 & $\begin{array}{l}\text { Strata exposed behind Shafer Ranch are fossil- } \\
\text { ferous, dolomitic, pelletal packstones. }\end{array}$ \\
\hline 0.2 & 58.5 & 21.6 & $\begin{array}{l}\text { Dry wash across Rocky Arroyo with massive traver- } \\
\text { tine deposits exposed on left. }\end{array}$ \\
\hline 0.5 & 59.0 & 21.1 & $\begin{array}{l}\text { Small cemetery on right. Cliffs to southeast } \\
\text { expose Seven Rivers Fm. fossiliferous, dolomitic, } \\
\text { pelletal packstones containing ostracodes, calci- } \\
\text { spheres, and some small foraminifers. Queen Fm. } \\
\text { (Shattuck Sandstone) is exposed at the cliff } \\
\text { base. }\end{array}$ \\
\hline 0.3 & 59.3 & 20.8 & $\begin{array}{l}\text { On the south side of the arroyo are cliffs } \\
\text { exposing thin-bedded dolomite of the Seven } \\
\text { Rivers Fm. dominated by pelletal wackestone } \\
\text { and mudstone. Stromatolitic(?) units have been } \\
\text { described from this locality (Sarg, 1976). } \\
\text { other, similar outcrops are along road on left. }\end{array}$ \\
\hline 0.5 & 59.8 & 20.3 & $\begin{array}{l}\text { Road cuts to left are Seven Rivers dolomite } \\
\text { (Dunham's (1972) Stop I-6). This section, } \\
11.7 \text { miles shelfward of the Capitan escarpment, } \\
\text { consists of thin-bedded, stromatolitic(?), } \\
\text { dolomitic wackestone with pellets, ostracodes, } \\
\text { and calcispheres. In and along the stream } \\
\text { valley, travertine is abundant along with } \\
\text { travertine-cemented gravels. } \\
\text { The dolomite in these sediments, as in most } \\
\text { of the back-reef areas, is very finely crystal- } \\
\text { line to aphanocrystalline and appears } \\
\text { to be of very early diagenetic origin. } \\
\text { Formation of early dolomite is probably } \\
\text { related to restricted circulation in these } \\
\text { back-reef lagoonal areas, precipitation of } \\
\text { CaSO minerals, and consequent increase in } \\
\text { Mg/Ca ratios of lagoonal and interstitial fluids. } \\
\text { Contact of Mg-rich surface and interstitial waters } \\
\text { with unstable aragonitic muds has led to partial } \\
\text { dolomitization of modern carbonate muds in the } \\
\text { Persian Gulf and this most likely took place } \\
\text { in the Permian back-reef areas as well. The } \\
\text { exact mechanisms of this dolomitization (reflux } \\
\text { brine movement, evaporative pumping, and other } \\
\text { models) are debated even in modern setting and } \\
\text { are even more disputed for the Permian examples. }\end{array}$ \\
\hline 0.9 & 60.7 & 19.4 & $\begin{array}{l}\text { Leaving Rocky Arroyo. Outcrops to the south are } \\
\text { upper Seven Rivers Fm. Yates Fm. is present on } \\
\text { the crest and eastern side of the hilis to the } \\
\text { south. }\end{array}$ \\
\hline 4.4 & 65.1 & 15.0 & $\begin{array}{l}\text { Junction with U.S. Highway 285; turn right toward } \\
\text { Carlsbad. The road here is on Quaternary } \\
\text { alluvium overlying Yates Fm. The Seven Rivers } \\
\text { Hills, to the northwest, are the type section } \\
\text { of the Seven Rivers Fm. (Meinzer and others, } \\
1926 \text { ). }\end{array}$ \\
\hline
\end{tabular}

stones and mudstones of probable lagoonal origin exposed on left. Note absence of evaporites and red beds. rata exposed behind Shafer Ranch are fossilferous, dolomitic, pelletal packstones. y wash across Rocky Arroyo with massive traverall cemetery on right. Cliffs to southeast expose Seven Rivers Fm. fossiliferous, dolomitic, pelletal packstones containing ostracodes, calcispheres, and some small foraminifers. Queen Fm. (Shattuck Sandstone) is exposed at the cliff

the south side of the arroyo are cliffs Rivers Fm. dominated by pelletal wackestone and mudstone. Stromatolitic(?) units have been described from this locality (Sarg, 1976). duts to left are Seven Rivers dolomite (Dunham's (1972) Stop I-6). This section, 11.7 miles shelfward of the Capitan escarpment, consists of thin-bedded, stromatolitic(?), dolomitic wackestone with pellets, ostracodes, and calcispheres. In and along the stream valley, travertine is abundant along with avertine-cemented gravels. The dolomite in these sediments, as in most line to aphanocrystalline and appears to be of very early diagenetic origin. related to restricted circulation in these back-reef lagoonal areas, precipitation of Caso 4 minerals, and consequent increase in Contact of $\mathrm{Mg}-\mathrm{rich}$ surface and interstitial waters with unstable aragonitic muds has led to partial dolomitization of modern carbonate muds in the in the Permian back-reef areas as we11. The exact mechanisms of this dolomitization (reflux brine movement, evaporative pumping, and other models) are debated even in modern setting and are even more disputed for the Permian examples. upper Seven Rivers Fm. Yates Fm. is present on the crest and eastern side of the hills to the Carlsbad. The road here is on Quaternary alluvium overlying Yates $\mathrm{Fm}$. The Seven Rivers of the Seven Rivers Fm. (Meinzer and others, 


\begin{tabular}{|c|c|c|c|}
\hline $\begin{array}{l}0.8 \\
0.9\end{array}$ & $\begin{array}{l}65.9 \\
66.8\end{array}$ & $\begin{array}{l}14 \cdot ? \\
13 \cdot 3\end{array}$ & $\begin{array}{l}\text { Bridge crossing over Rocky Arroyo. } \\
\text { Interbedded dolomites and red-brown sandstone } \\
\text { of the Yates Fm. on both left and right. }\end{array}$ \\
\hline 0.6 & 67.5 & 12.7 & Yates $\mathrm{Fm}$. exposed in road cut. \\
\hline 0.3 & 67.8 & 12.4 & Yates $\mathrm{Fm}$. exposed in road cut. \\
\hline 1.5 & 69.2 & 10.9 & $\begin{array}{l}\text { Intersection of US Highway } 285 \text { with US } 285 \\
\text { (Truck Route) on right. Continue left on } \\
\text { main US Highway } 285 .\end{array}$ \\
\hline 0.3 & $69 \cdot 5$ & $10 \cdot 6$ & $\begin{array}{l}\text { Basal Tansill Fm. is exposed in low cuts on both } \\
\text { sides of road. Thin-bedded to laminated dolo- } \\
\text { mitic mudstones with evaporite (anhydrite?) } \\
\text { crystal casts can be found just below road } \\
\text { level to the west. All back-reef units dip } \\
\text { gently basinward in this area. }\end{array}$ \\
\hline 0.4 & 69.9 & 10.2 & $\begin{array}{l}\text { The 0cotillo Hills at } 1: 000^{\prime} \text { clock and Avalon } \\
\text { Hills at } 11: 00 \text { to } 12: 000^{\prime} \text { clock are composed } \\
\text { of back-reef Tansill and Yates evaporitic } \\
\text { dolomites. The hills are the topographic } \\
\text { expression of an anticlinal structure over } \\
\text { the buried Capitan reef, one of many such } \\
\text { structures in the shelf area to the north and } \\
\text { west of Carlsbad. }\end{array}$ \\
\hline 1.9 & 71.8 & $8 \cdot 3$ & $\begin{array}{l}\text { Thin-bedded Tansill dolomites with evaporite } \\
\text { crystal casts present in roadcut. The type } \\
\text { locality of the Tansill Fm., as described by } \\
\text { DeFord and Riggs (1941), is located nearby in the } \\
\text { Ocotillo Hills. }\end{array}$ \\
\hline 0.4 & 72.2 & 7.9 & $\begin{array}{l}\text { Gently dipping Tansill and Yates sediments in } \\
\text { canyon to right are on eastern flank of the } \\
\text { Tracy Dome. }\end{array}$ \\
\hline 1.1 & $73 \cdot 3$ & 6.8 & $\begin{array}{l}\text { Tansill dolomite overlying Yates sandstone is } \\
\text { exposed in canyon on right. }\end{array}$ \\
\hline 0.7 & 74.0 & 6.1 & $\begin{array}{l}\text { Pecos River on left. Tansill Fm. in road cuts on } \\
\text { right. }\end{array}$ \\
\hline 0.8 & 74.8 & 5.3 & Living Desert State Park turn-off on right. \\
\hline 3.1 & 77.9 & 2.2 & $\begin{array}{l}\text { Carlsbad city center (junction of US Highways 62- } \\
180 \text { (from north) and 285); La Caverna Hotel). } \\
\text { Continue straight ahead. }\end{array}$ \\
\hline 2.2 & 80.1 & 0.0 & $\begin{array}{l}\text { Intersection of US Highways } 62-180 \text { (from south) } \\
\text { and } 285 \text { (from southeast). } \\
\text { Roadlog ends. }\end{array}$ \\
\hline
\end{tabular}




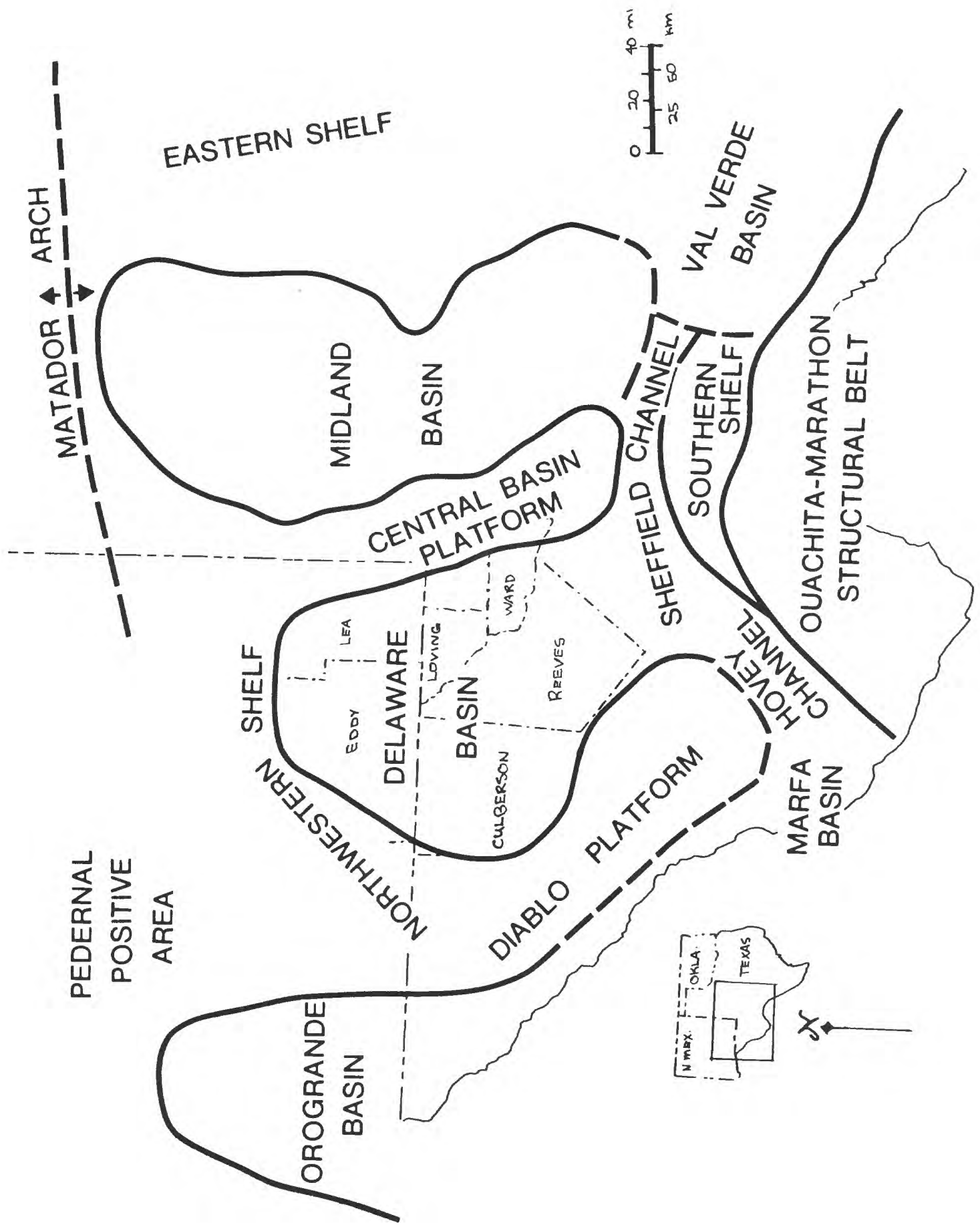

Figure 1.--Pennsylvanian and Permian physiographic features in the Permian Basin region. Modified from King (1943), lickee, Oriel and others (1967), and Williamson (1979). 

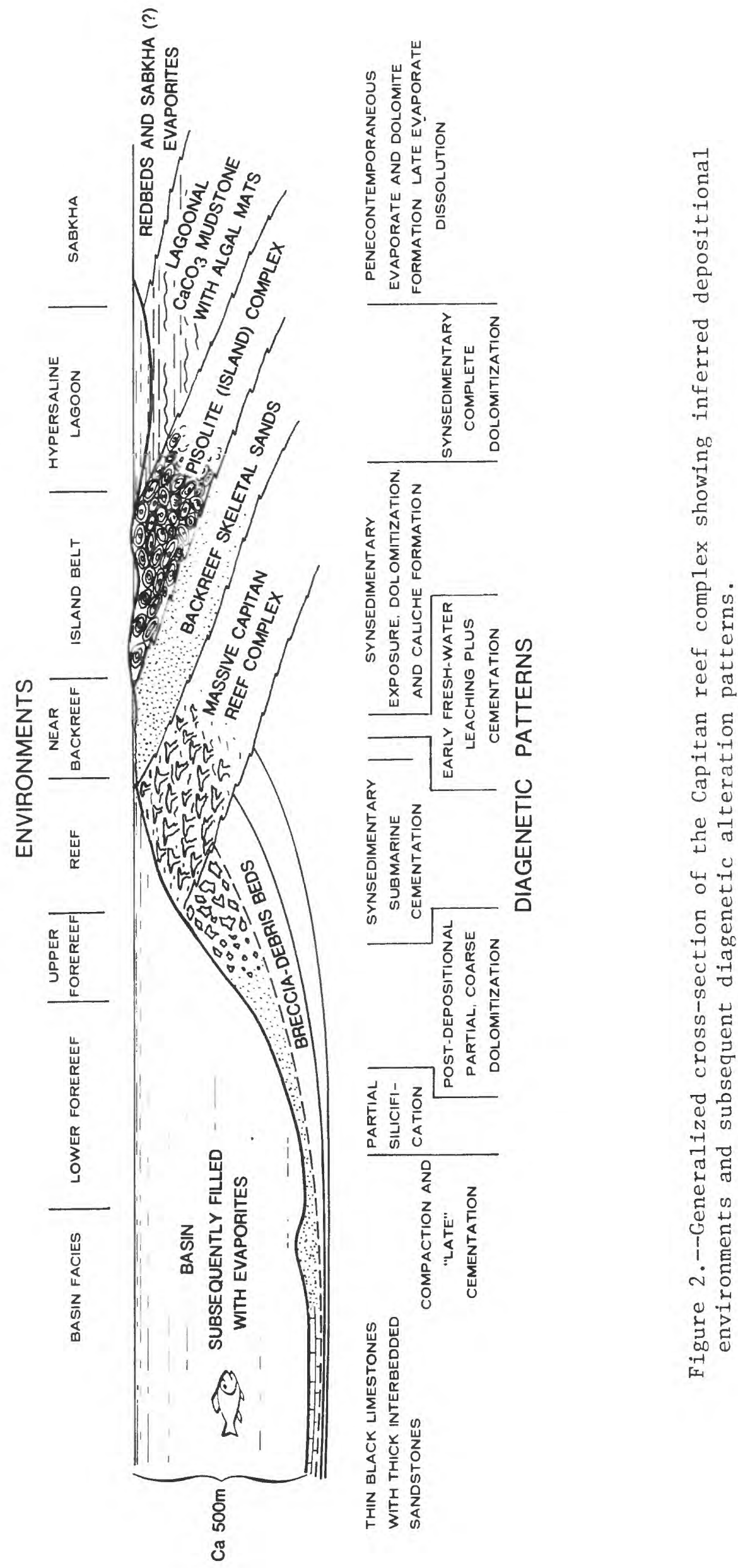


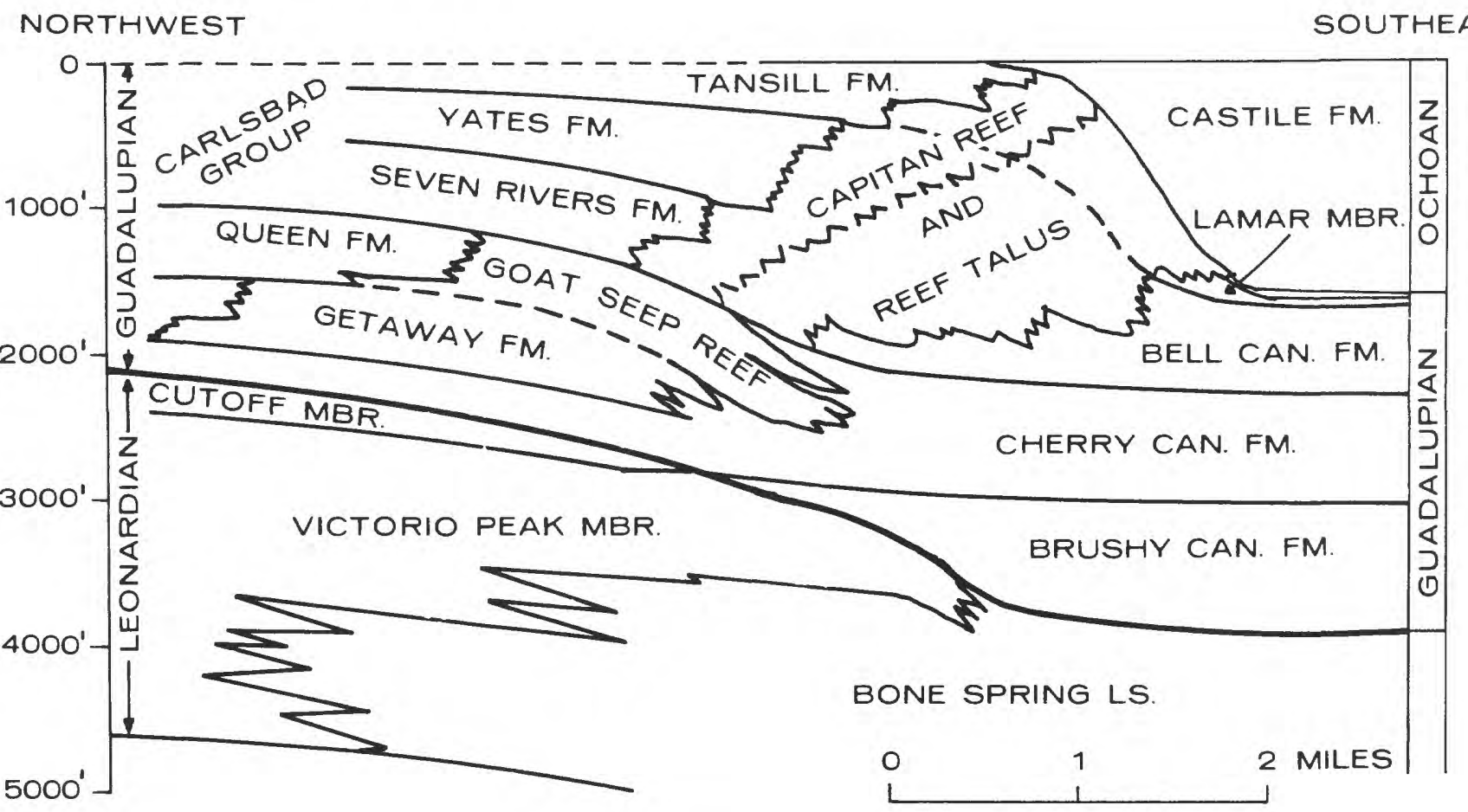

AFTER KING 1948, NEWELL, ET. AL. 1953, HAYES 1957. AND TYRRELL 1964

Figure 3.--Stratigraphic nomenclature and inferred facies relationships for Leonardian, Guadalupian, and Ochoan units of the Northwest Shelf and Delaware Basin. 


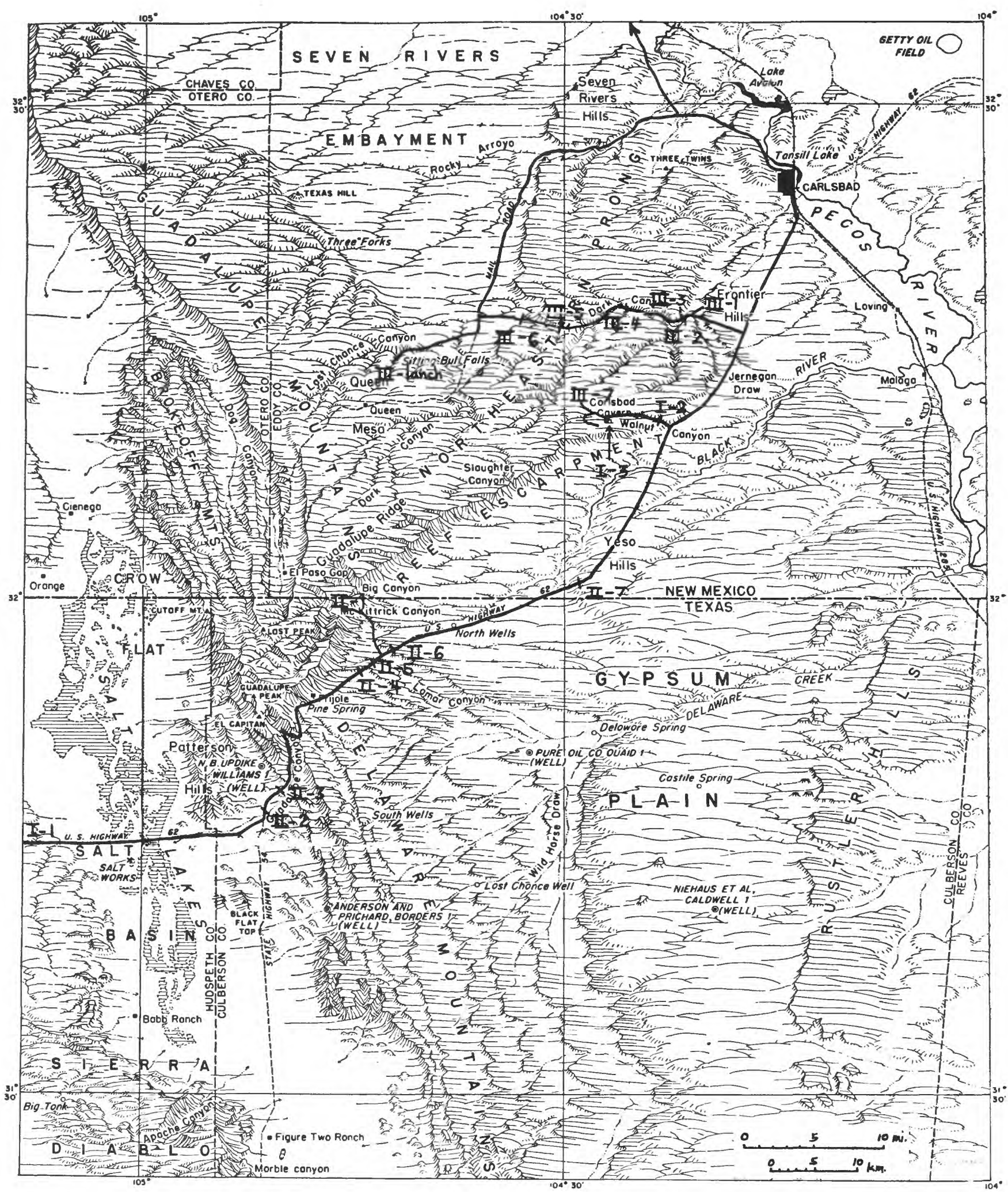

Figure 4.--Physiographic diagram of the Guadalupe-Delaware Mountains area showing the routes covered on this field trip and stop locations keyed to the roadlog. Adapted from King, 1948. 


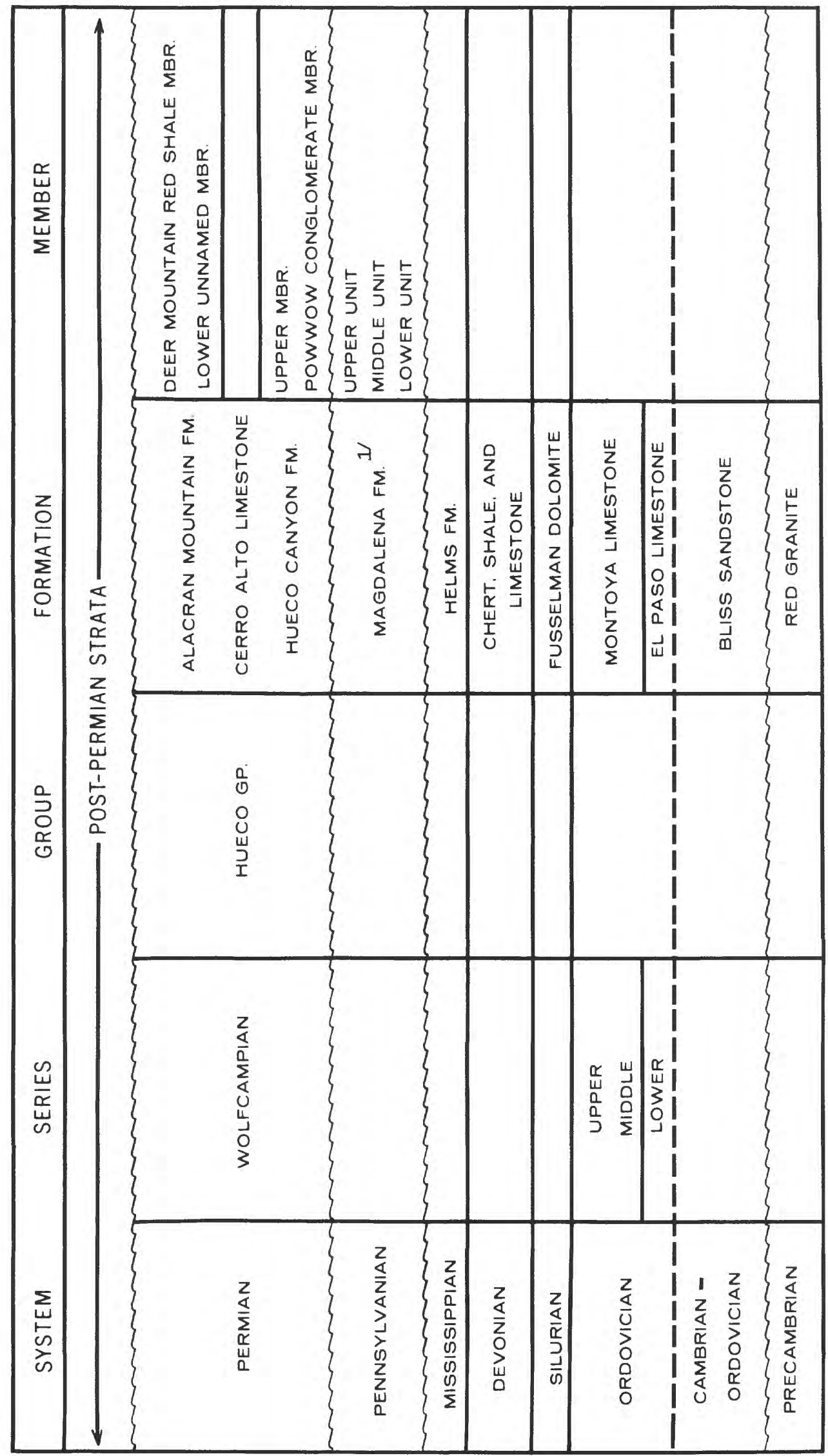

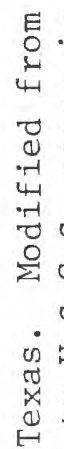

0
0
0
0
0
0
0
0
0
0
0
0
0
0
0
0
0
0
0
0
0
0
0

i 雚

点藏.

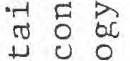

节范

욤ำ

氙可

(1) के 0

d की 0

4 웅

每

둬

$\Rightarrow$

as.

$+100$

$\pi$

Dᄃ $\mathrm{E}$

บ क ?

0

N

is

$\sum_{0}^{0} \underset{0}{0} \frac{\pi}{d}$

范

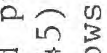

छ

N $=7$

तो क

(c)

덩 吉

100

! 号 U

(1) of 2

品望告 


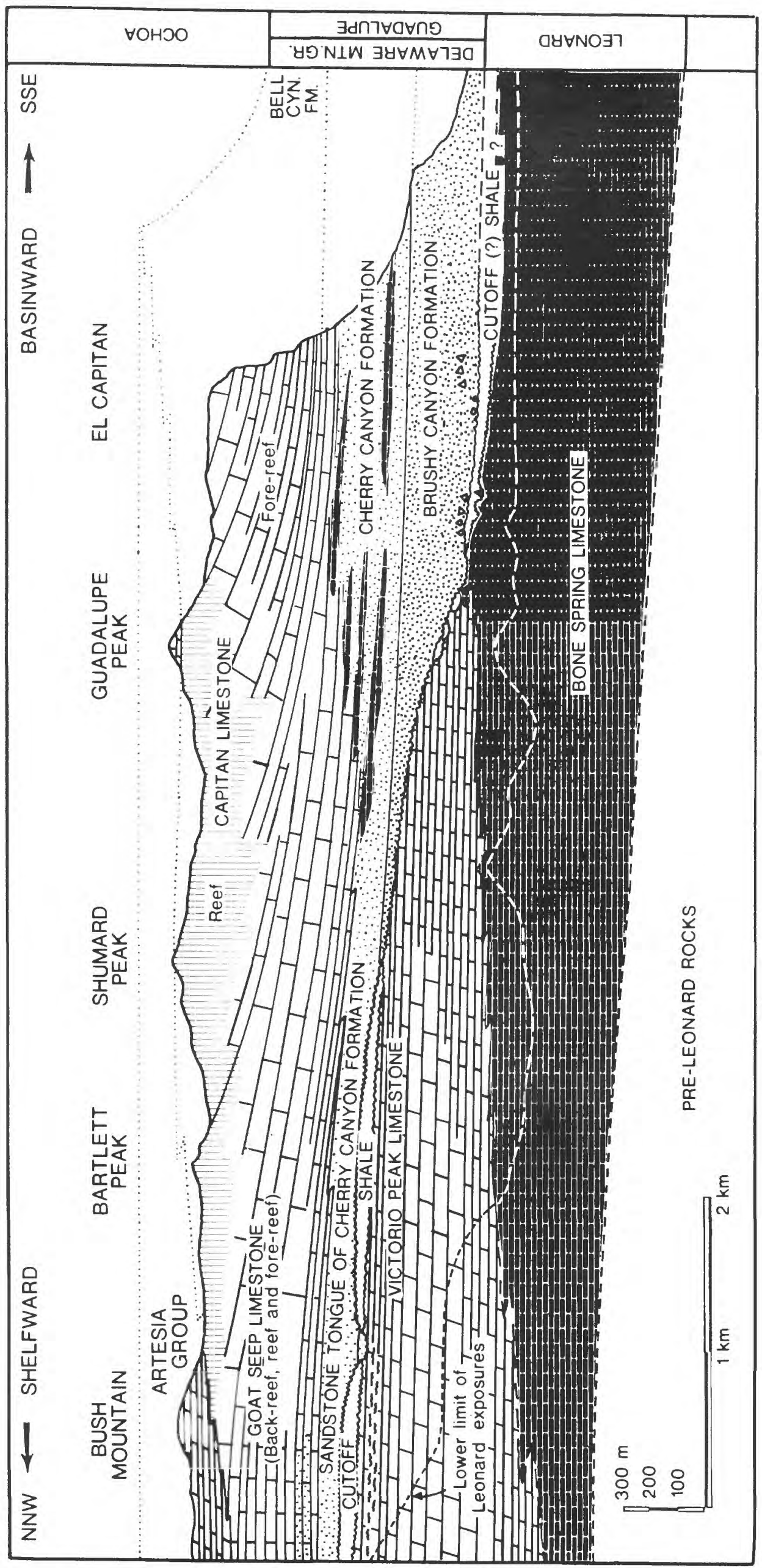

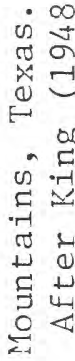

ป

넘 1

릉

E 药

(1)

क. 우

34

㝴虫

วิ

is

$\stackrel{2}{2}$

$+1$

फ़

E 8

- 13

(0)

.

章告

告 0

$2 \pi$

.

1.

I

응

6 वे

虽 牙 


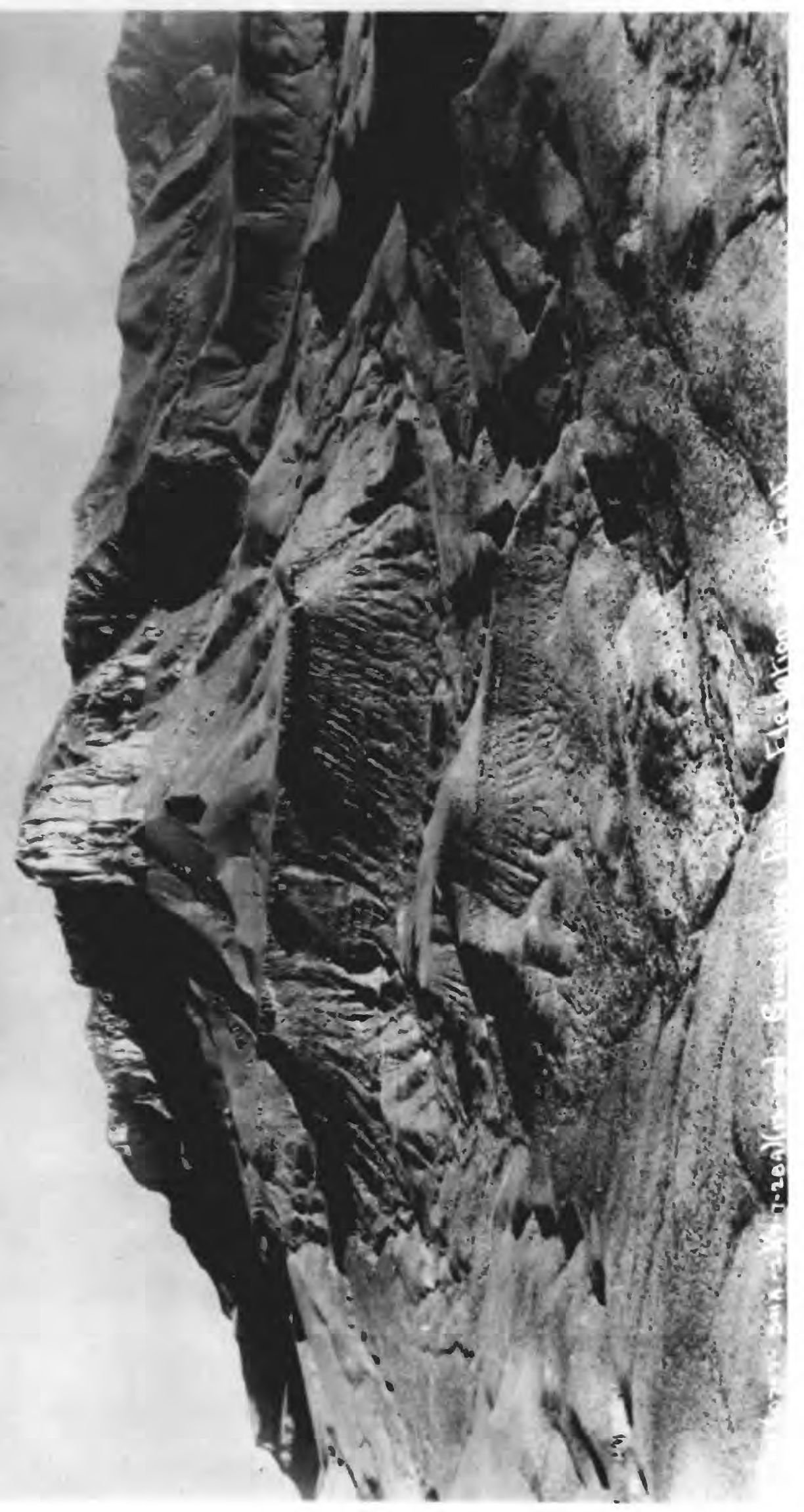

웅

길

ชั

可战

○ी 믇

ㄷํㅇ

त् 1

도 농

य

a

ช․ㄷㄷㄷㄴ

도을

包是 U

击

馬

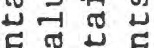

곤

出完远

(1) त

웡

击

ฮึ

刃ํ-1

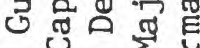

\&

W

出品出

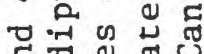

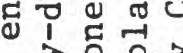

덕엄ㄹ

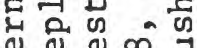

\& ${ }^{\infty}$ J

जैन्न ब्च

का ए 0

$0 \stackrel{0}{\square}$

过罂的武

की 0 .

岹藏

3 ह

क

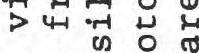

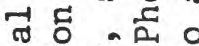

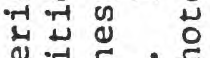

ఖँ

व लू Нै

च्र त 0

可山品

न

응 00 出

1 死。

- ती

วิ

u

ไั ฮึ

of 4 U 点 


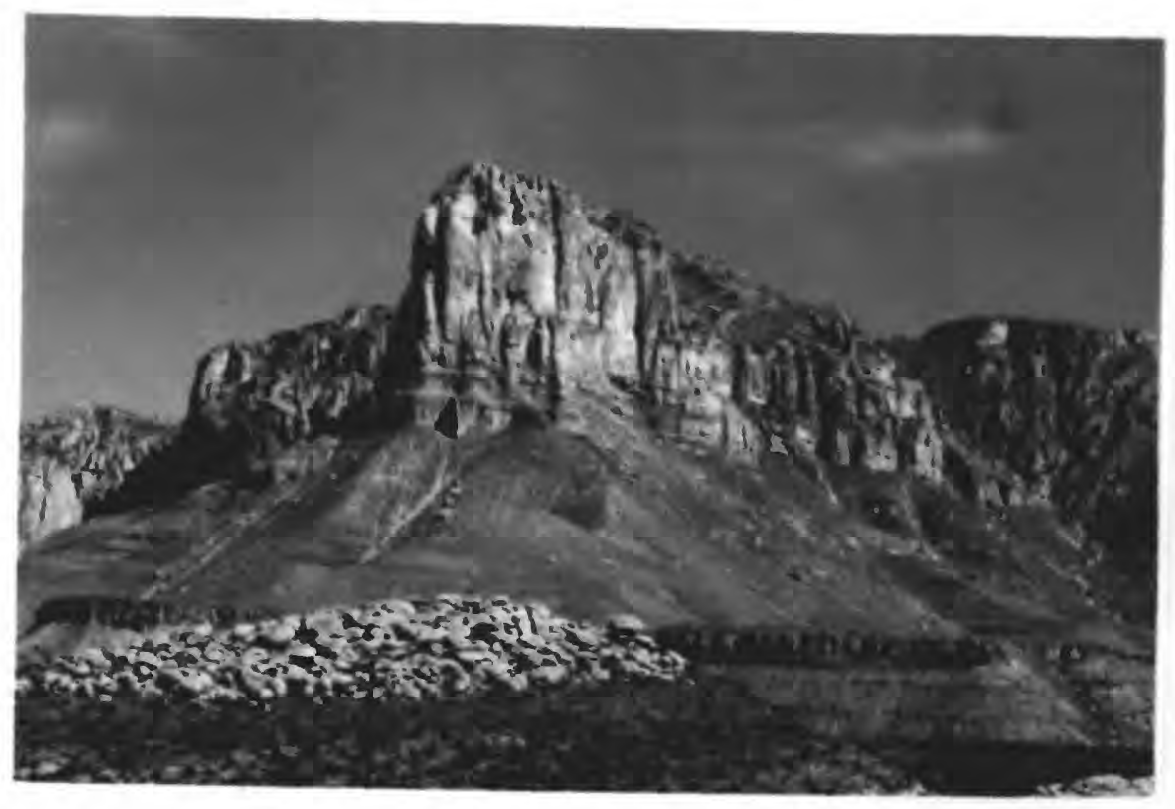

Figure 8.--View of the southern end of the Guadalupe Mountains. Note massive Capitan limestones of E1 Capitan (top center). underlying basinal sandstones, siltstones, and shales of the Cherry Canyon and Brushy Canyon Formation: and the lenticular, sandstone-filled channel in the Brushy Canyon in the foreground.

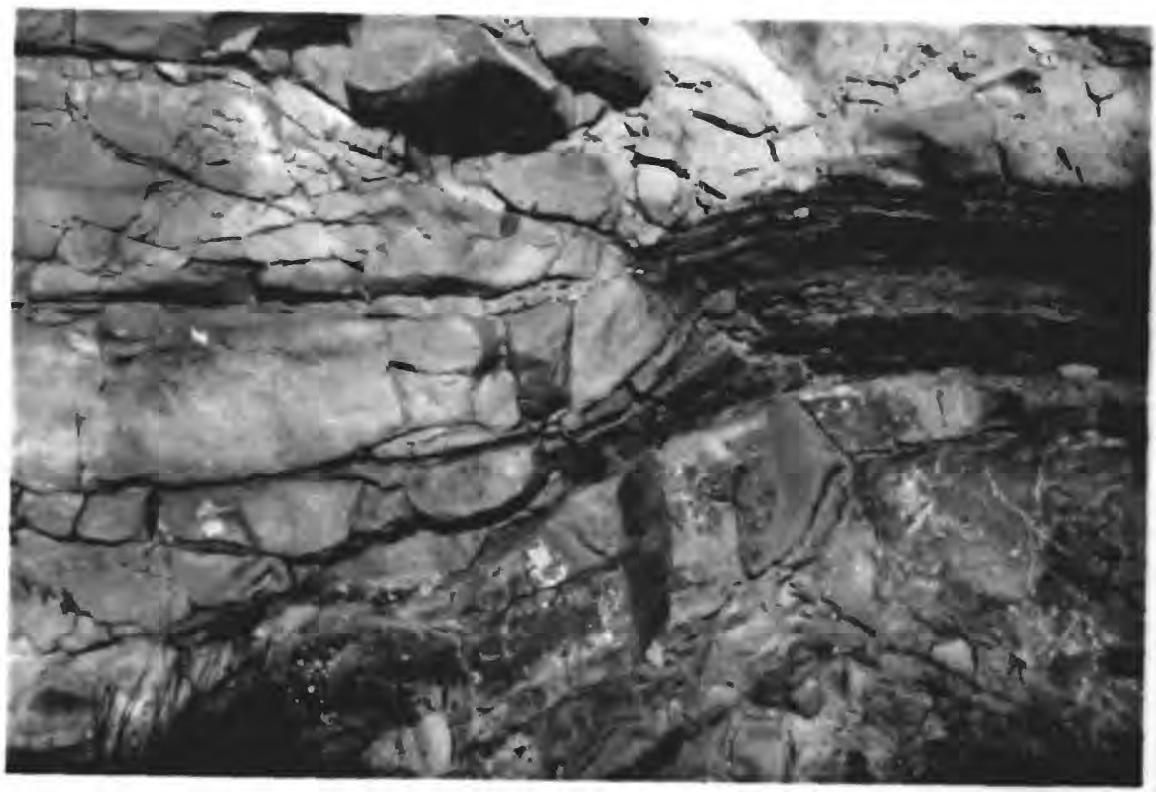

Figure 9.--Margin of sandstone-filled submarine channel in the Brushy Canyon Formation. This exposure (Stop II-3) illustrates overbank siltstones and shales (on right) cut and filled by a massive, fine-grained sandstone. Note injection structures at sandstoneshale contact.

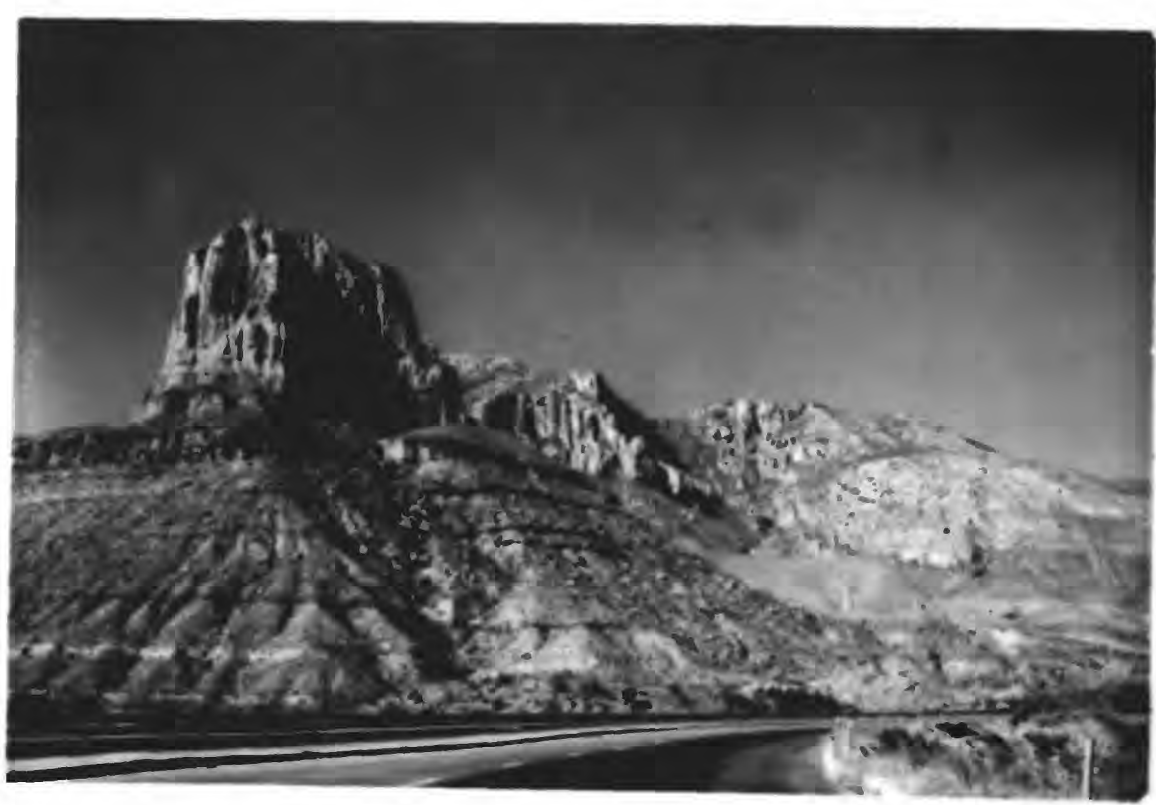

Figure 10.--View of E1 Capitam from roadside rest area (mileage 101.7 on E1 PasoCarlsbad roadlog). Hill in left foreground consists of lenticular sandstones Interbedded with siltstones and shales (Brushy Canyon Formation). The Cherry Canyon Formation forms the recessive slope between the top of the foreground cliff and the massive, steeply-dipping Capitan limestones of E1 Capitan. Note the bank-to-basin transition in the right half of the photo (dips flattening to the east into the Delaware Basin). 

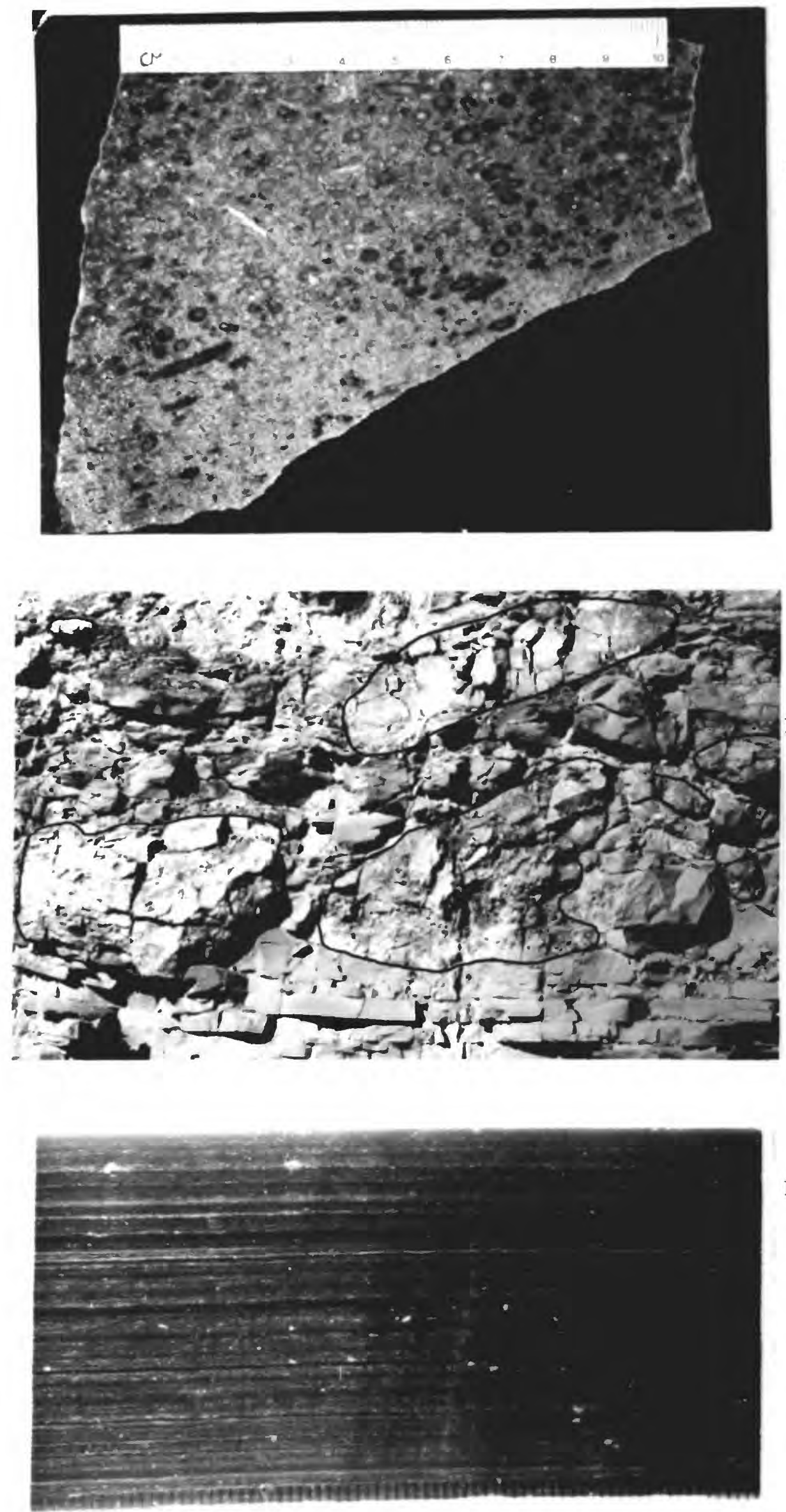

Figure 11.--Oriented fusulinid Foraminifera in basinal sandstone of the upper Brushy Canyon Formation. Fusulinids were reworked Irom the shelf and oriented by submarine currents. Locality is on U.S. Highway $62-180$ at mileage 102.8 on E1 PasoCarlsbad roadlog.

Figure 12.--Large, rounded, reef-derived limestone clasts of the Rader slide which have foundered into a matrix of Bell Canyon sandstone. This location (Stop II-4) exposes part of a thin, channelized submarine debris flow or slide which has carried reef material at least five miles into the basin.

Figure 13.--Close-up photograph of a polished sample of the basinal Lamar Limestone Member of the Bell Canyon Formation (locality at Stop II-6). Note very sma11scale, uniform lamination (small divisions at bottom of photo are millimeters) and dark color of sediment. 

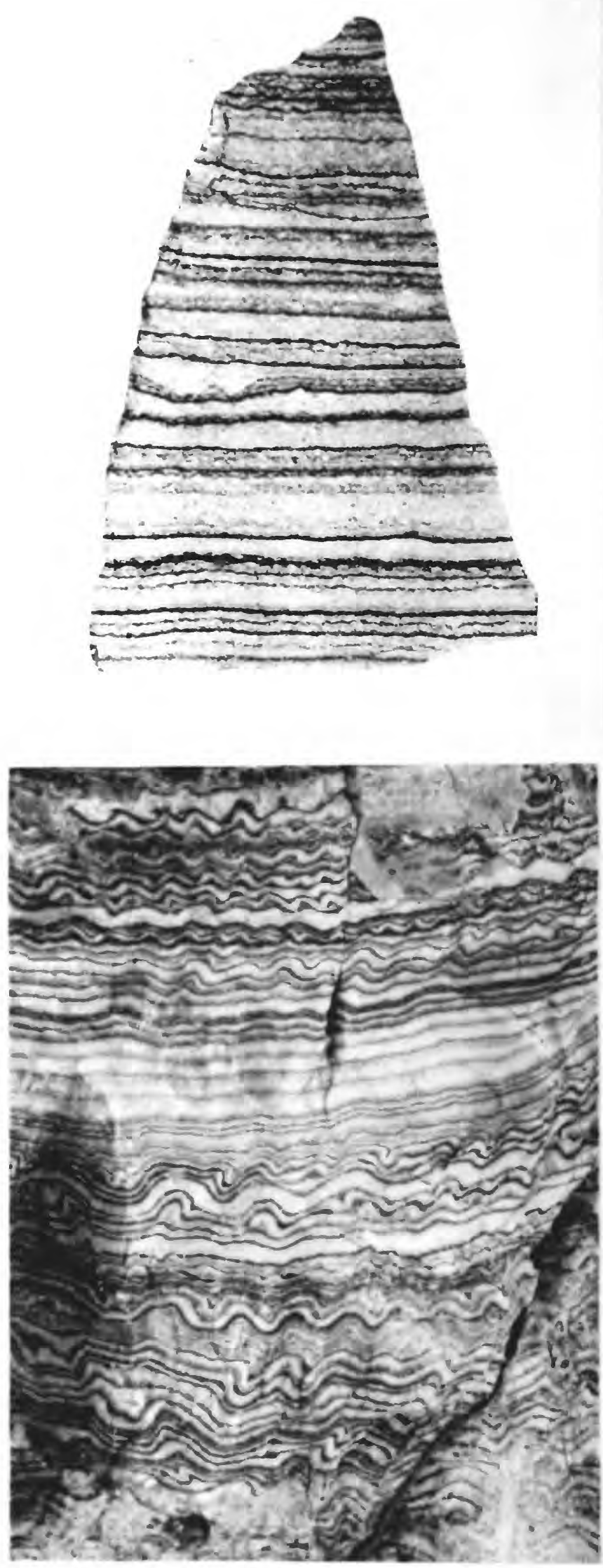

Figure 14.--Photograph of a thin-section of laminated anhydrite from the castile Formation ( $2 x$ magnification). The sample was collected from surface outcrops about 20 miles east of El

Capitan. Photo from King (1948, plate 10-B). Note the regular, thin laminations which consist of alternations of anhydrite and (or) gypsum (light layers) and an organic matter-calcite mixture (dark layers). Although some small-scale discontinuities can be seen in this photo in some laminae, packages of these laminae have been traced laterally for greater than 70 miles.
Figure 15.--Small-scale contortions in laminated gypsum from Castile Formation. These structures are probably produced as a consequence of rehydration of anhydrite to gypsum during uplift and weathering. Sample from stop II-7; vertical axis of photograph represents approximately 3 feet on outcrop. 


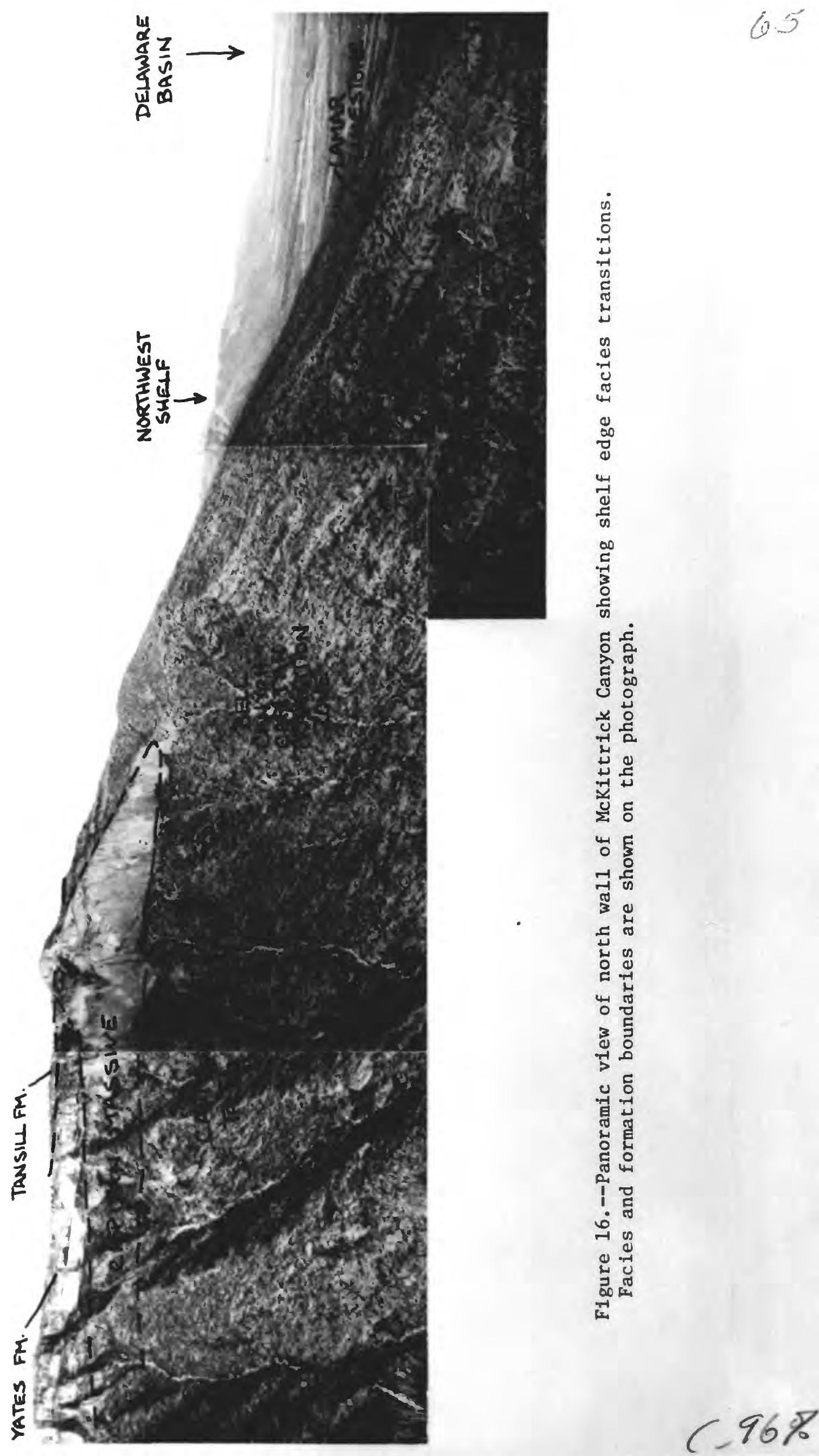



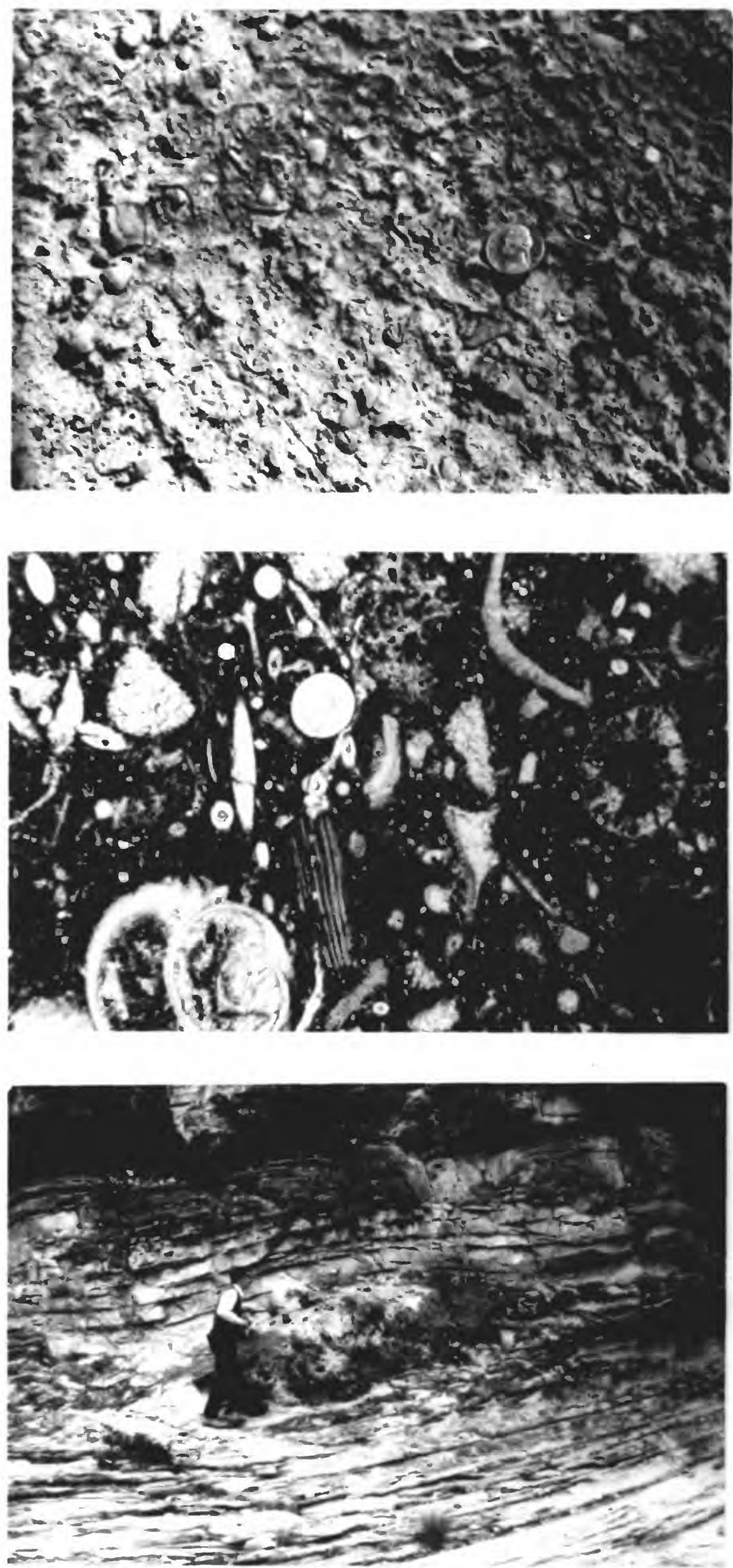

Figure 17.--View of bedding plane in toe-of-slope facles of the lamar Limestone Member of the Bell Canyon Formation. Brachlopods are extremely abundant but bryozoans, echinoderms, and siliceous sponges are also commonly found. The originally calcitic fauna has been largely silicifled in this area. Sample from mouth of Mckittrick Canyon.

Figure 18.--Photomicrograph of toe-of-slope limestone from McKittrick Canyon. Note mixture of reef and slope fauna including abundant siliceous sponge spicules set in micritic matrix. Long axis of photo equals approximately $2.5 \mathrm{~mm}$.

Figure 19.--Compactional drape in toe-of-slope limestones surrounding a large block of reefderived Iimestone. Rader Member of Bell Canyon Formation at mouth of McKittrick Canyon. 

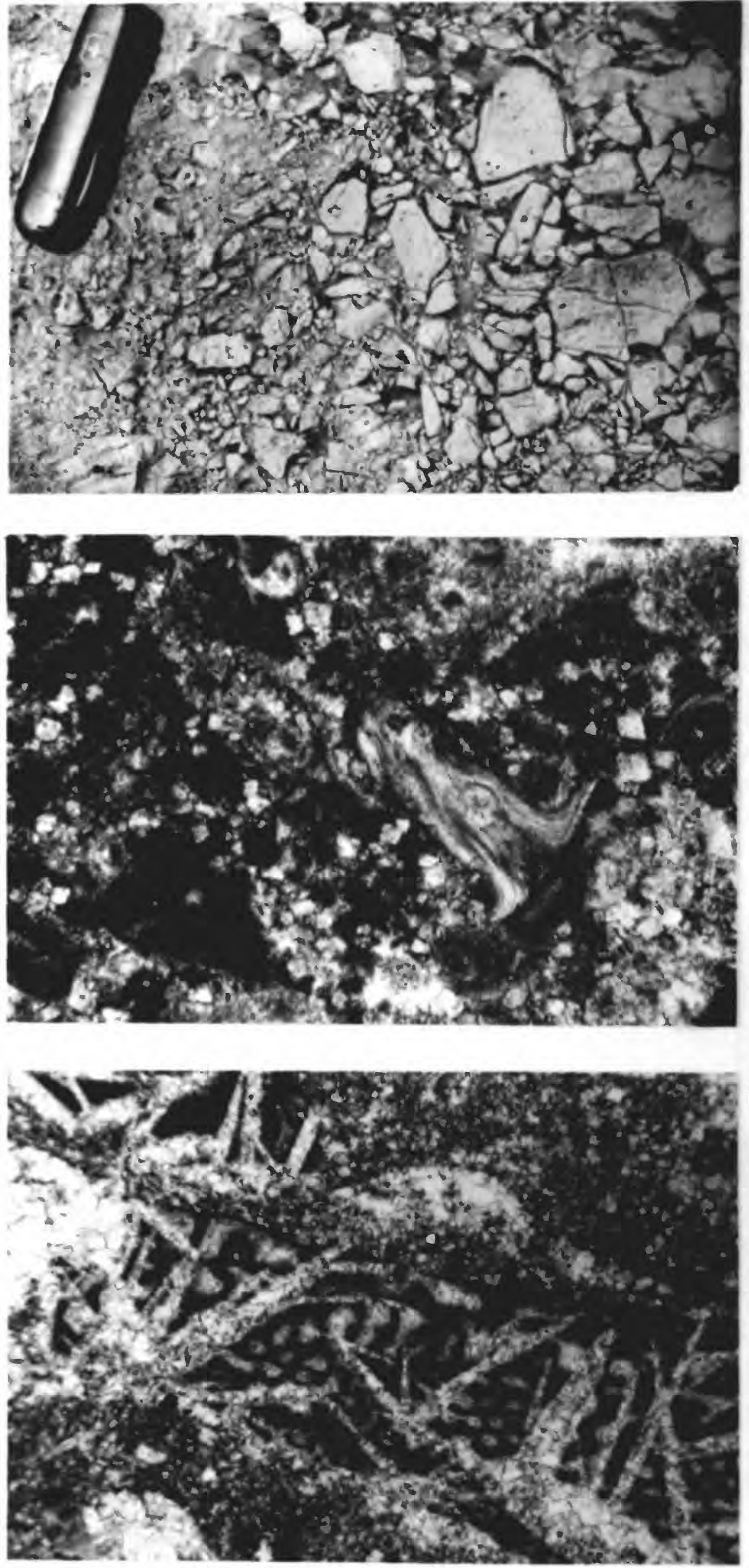

Figure 20.--Angular, reefderived rubble in the Capitan talus facies, Mckittrick Canyon. Although some in-situ brecclation has taken place, much of this material consists of reef limestone, fragmented and redeposited in this forereef setting. Knife is approximately 3 inches long.

Figure 21.--Photomicrograph of typical Capitan talus facies. Note pellets, bryozoan fragments, and extensive but incomplete dolomitization of matrix but not framework grains. Long axis of photo equals approximately $2.5 \mathrm{~mm}$.

Figure 22.--Photomicrograph of Capitan talus facies in Mckittrick Canyon. Note in-situ fracturing of shelf-derived fusulinid foraminifer and extensive dolomitization of micritic matrix. Long axis of photo equals approximately $2.5 \mathrm{~mm}$. 

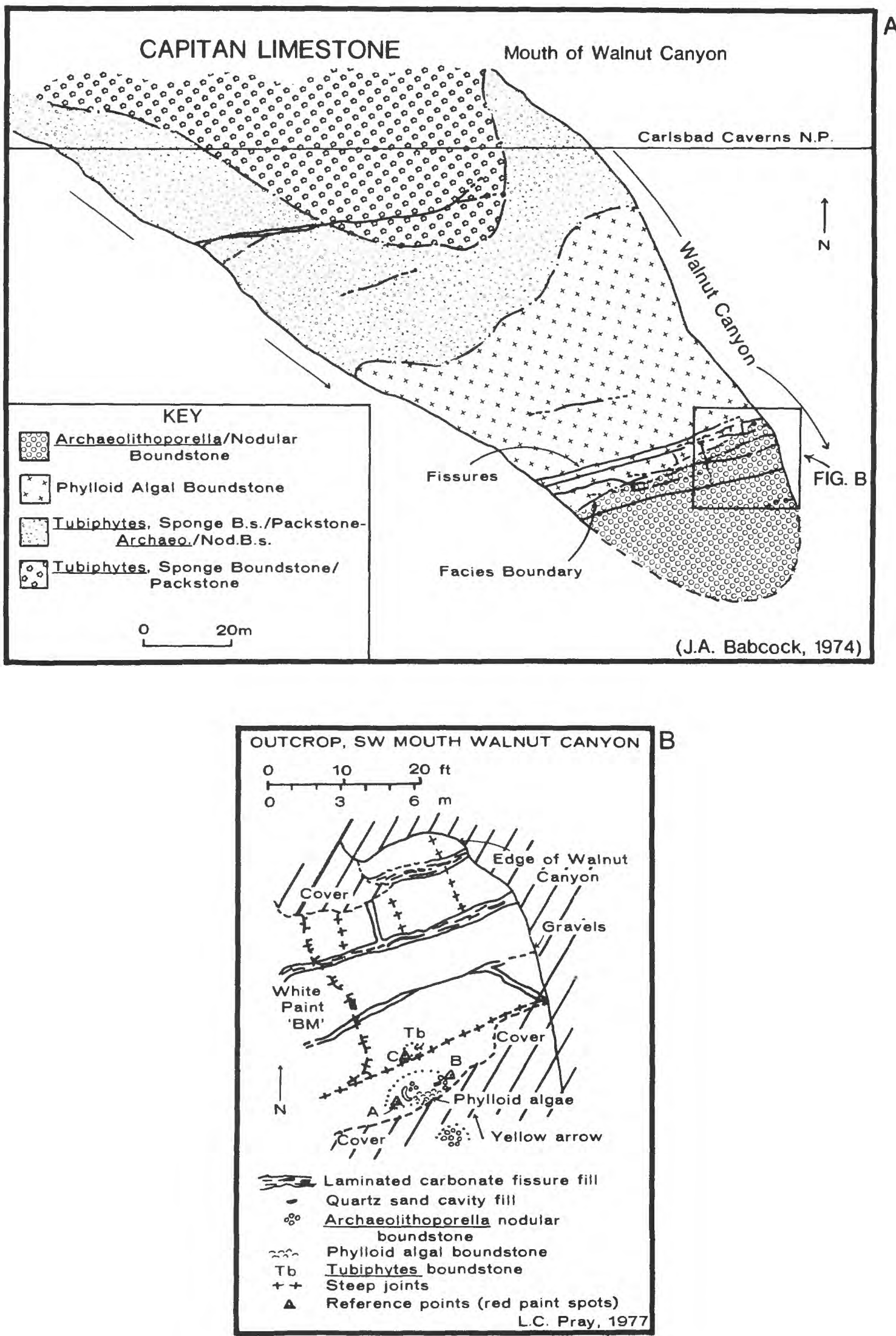

Figure 23.--Microfacies exposed at the mouth of Walnut Canyon (Stop I-2). After Babcock, J.A. (1977). 

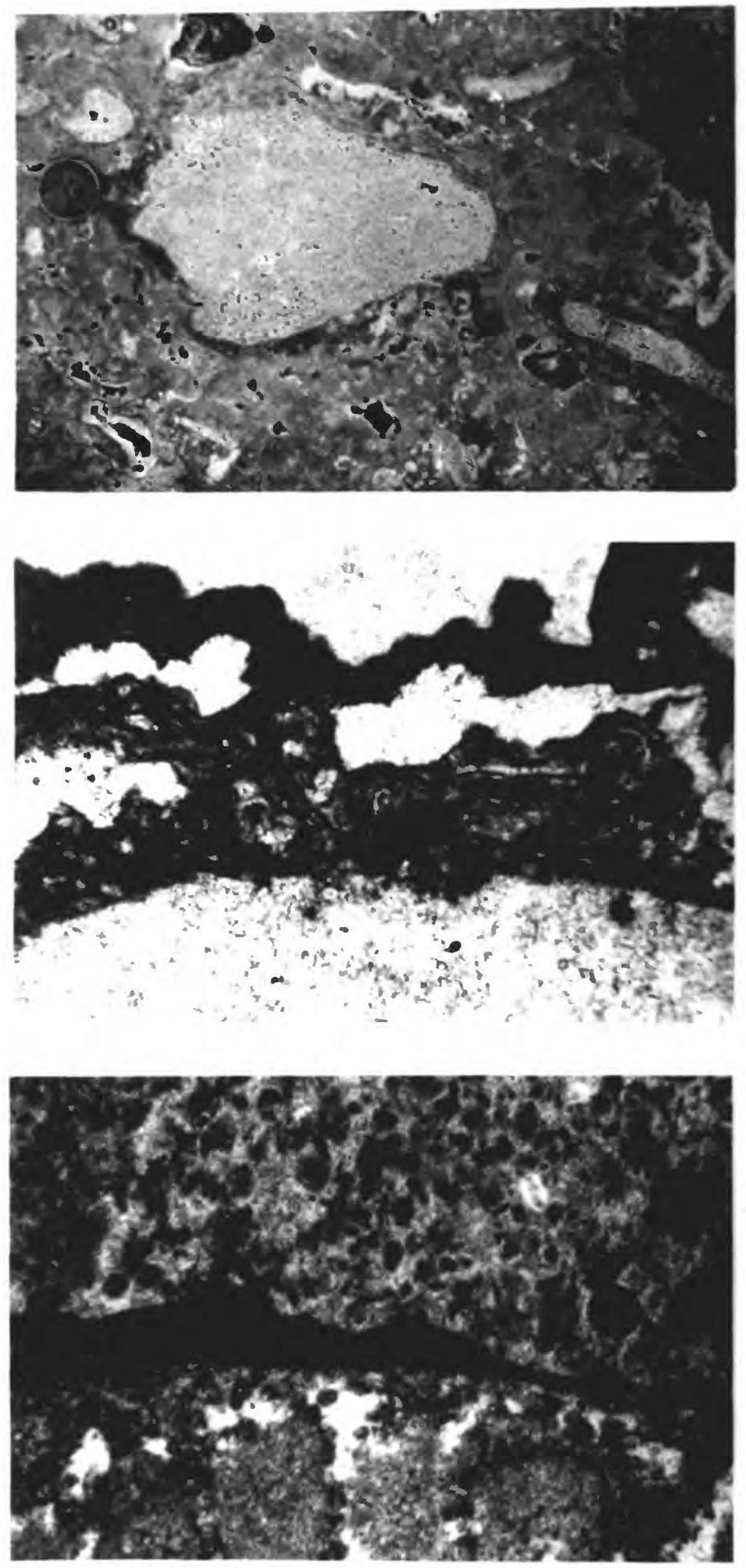

Figure 24.--Closeup of reef fabric near the mouth of Walnut Canyon (along north side of Bat Cave Canyon). Note oriented, in-place sponges surrounded by darker algal encrustations; porosity largely fllled with fibrous, submarine cements.

Figure 25.--Phot omicrograph of Capitan reef facies. Lower part of photo shows a largely neomorphosed calcareous sponge; this is overlain by numerous Archaeolithoporella encrustations (dark colored) interlayered with submarine cements (1ight colored). Long axis of photograph equals approximately $2.5 \mathrm{~mm}$.

Figure 26.--Photomicrograph of Capitan reef facies. Lower half of photo shows a largely neomorphosed calcareous sponge; this is overlain by irregular, dark, algal encrustations. Remnant porosity filled by pelletal, penecontemporaneous, internal sediment. Same scale as previous photo. 


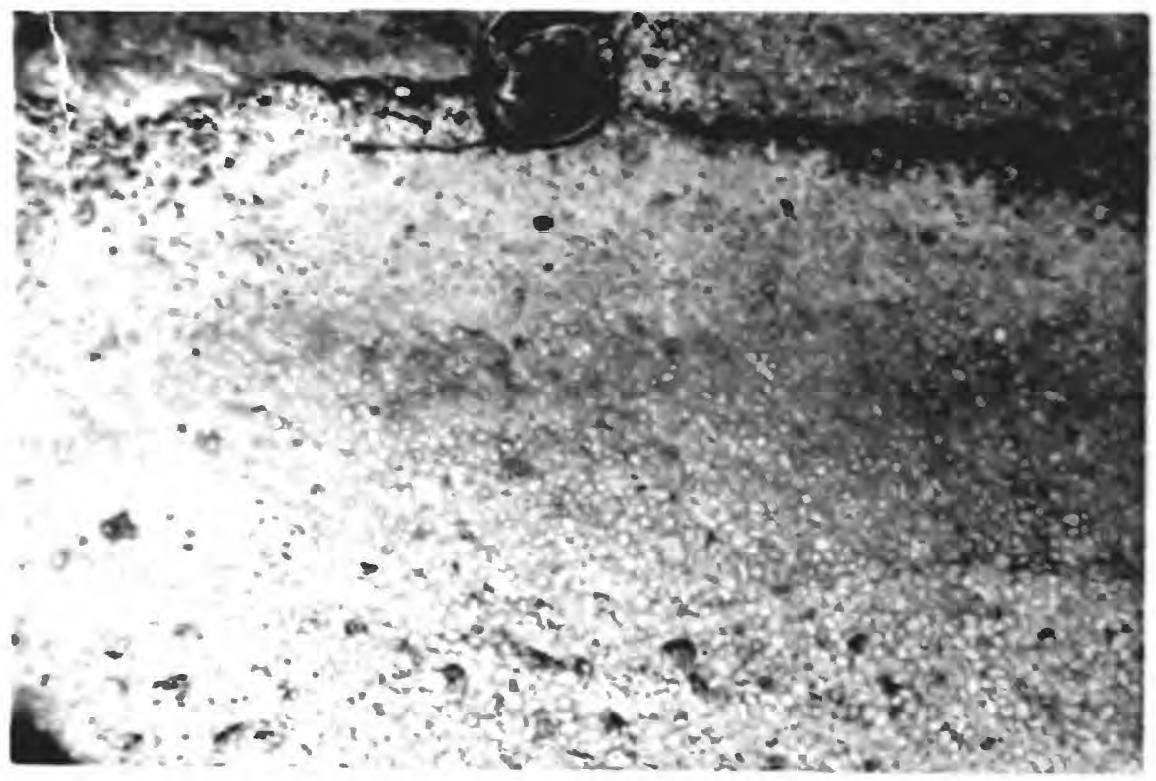

Figure 27.--Closeup of oolitic grainstone in Walnut Canyon. Grain nuclei are mainly green algae. Note excellent sorting and rounding of grains and general lack of internal sedimentary structures.

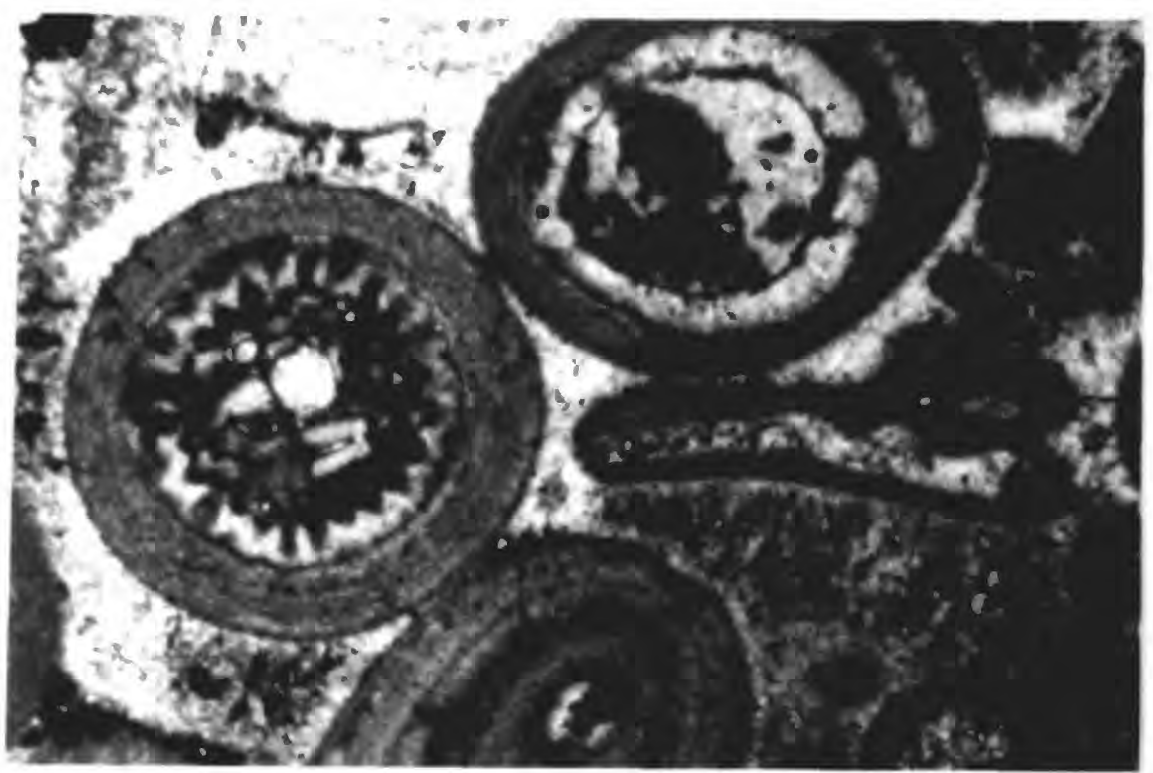

Figure 28.--Photomicrograph of grainstone shown in figure 27. Note nuclei of Mizzia green algae; these are oolitically coated and then surrounded by submarine cements. Long axis of photo equals approximately $2.0 \mathrm{~mm}$.

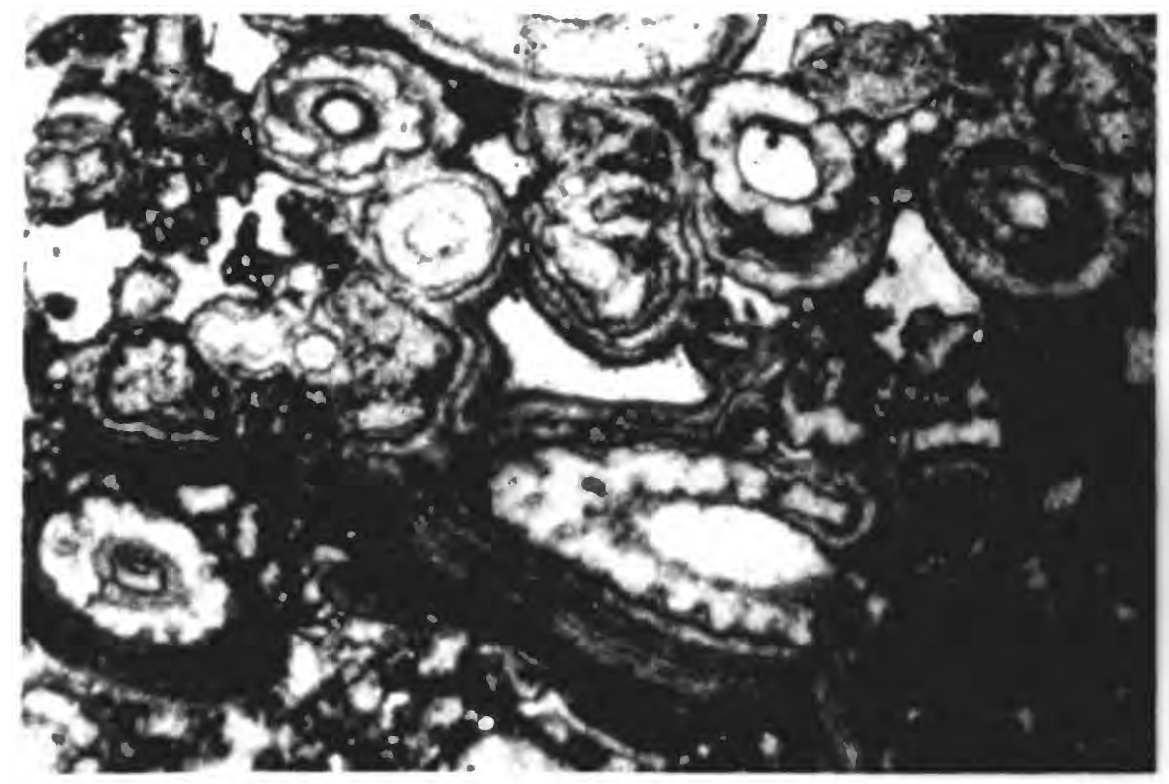

Figure 29.--Photomic rograph of grainstone from Walnut Canyon. Virtually a11 grains are altered green algae; note extensive encrustation and binding of grains into "grapestones". Same scale as previous photo. 

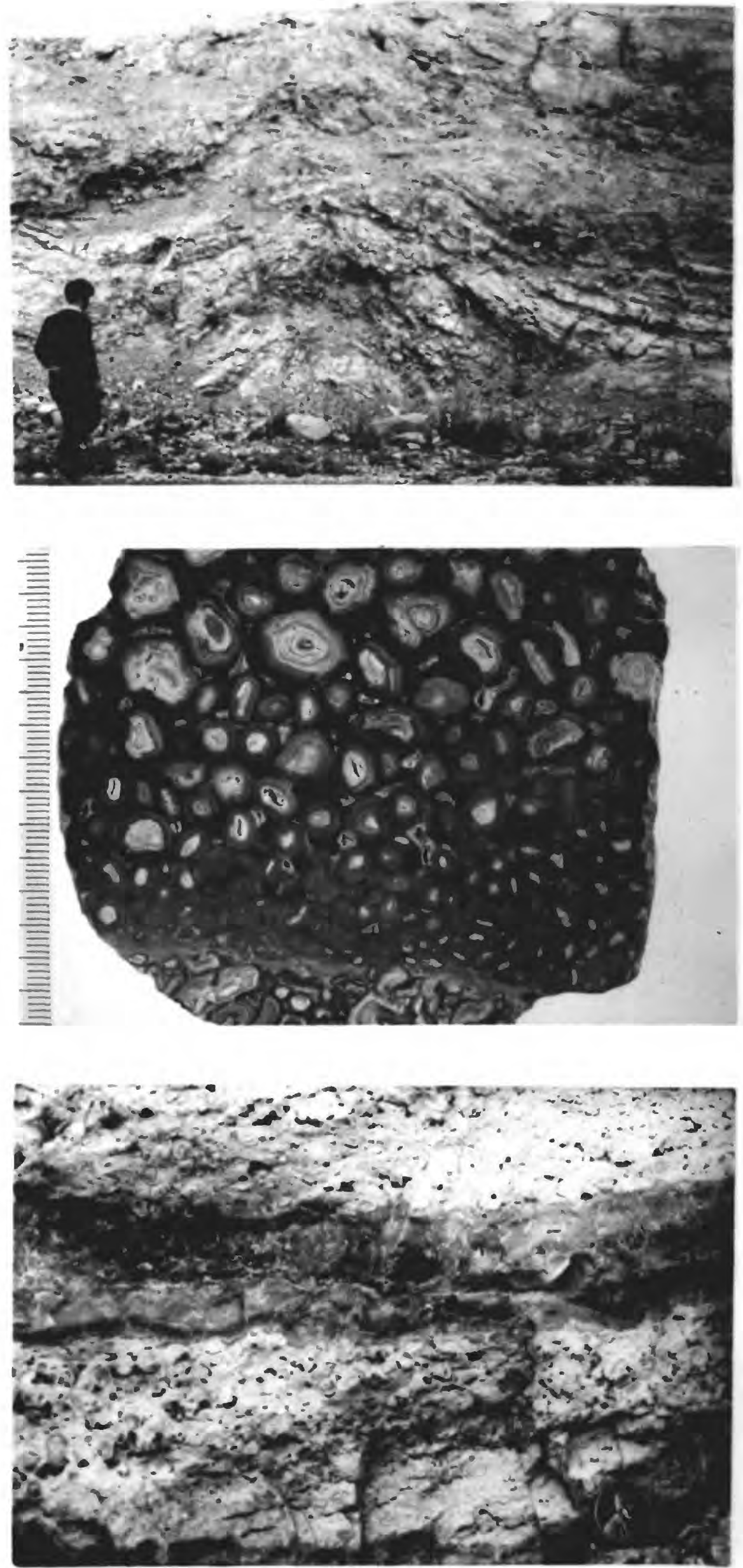

Figure 30.--Tepee structure in Walnut Canyon (Stop II I-7). Sediments are pisolitic dolomites of the Yates Formation.

Figure 31.--Closeup of a polished slab of pisolitic dolomite from Walnut Canyon. Note well deve1oped reverse grading, abundant nuclei of fractured pisolites, and excellent preservation of small-scale concentric laminations by replacement dolomite. Small scale divisions at left are millimeters.

Figure 32.--Pisolitic sediments of Yates Formation cut by a large, horizontal, sheet crack of calcite spar. Sheet crack is about 6 inches high. 


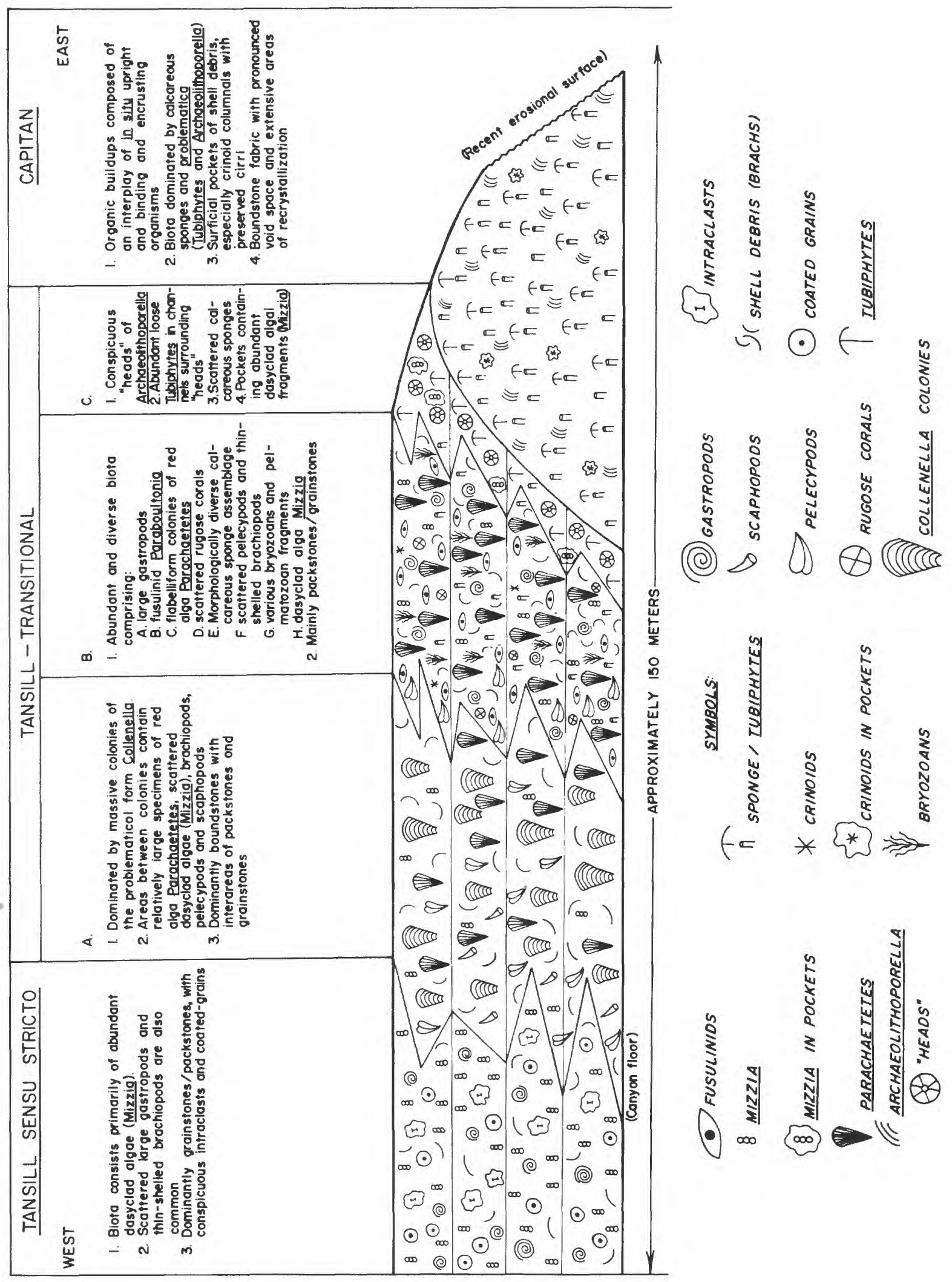

Figure 33.--Microfacies exposed at the mouth of Dark Canyon (Stop III-1). From Toomey and Cys (1977). 


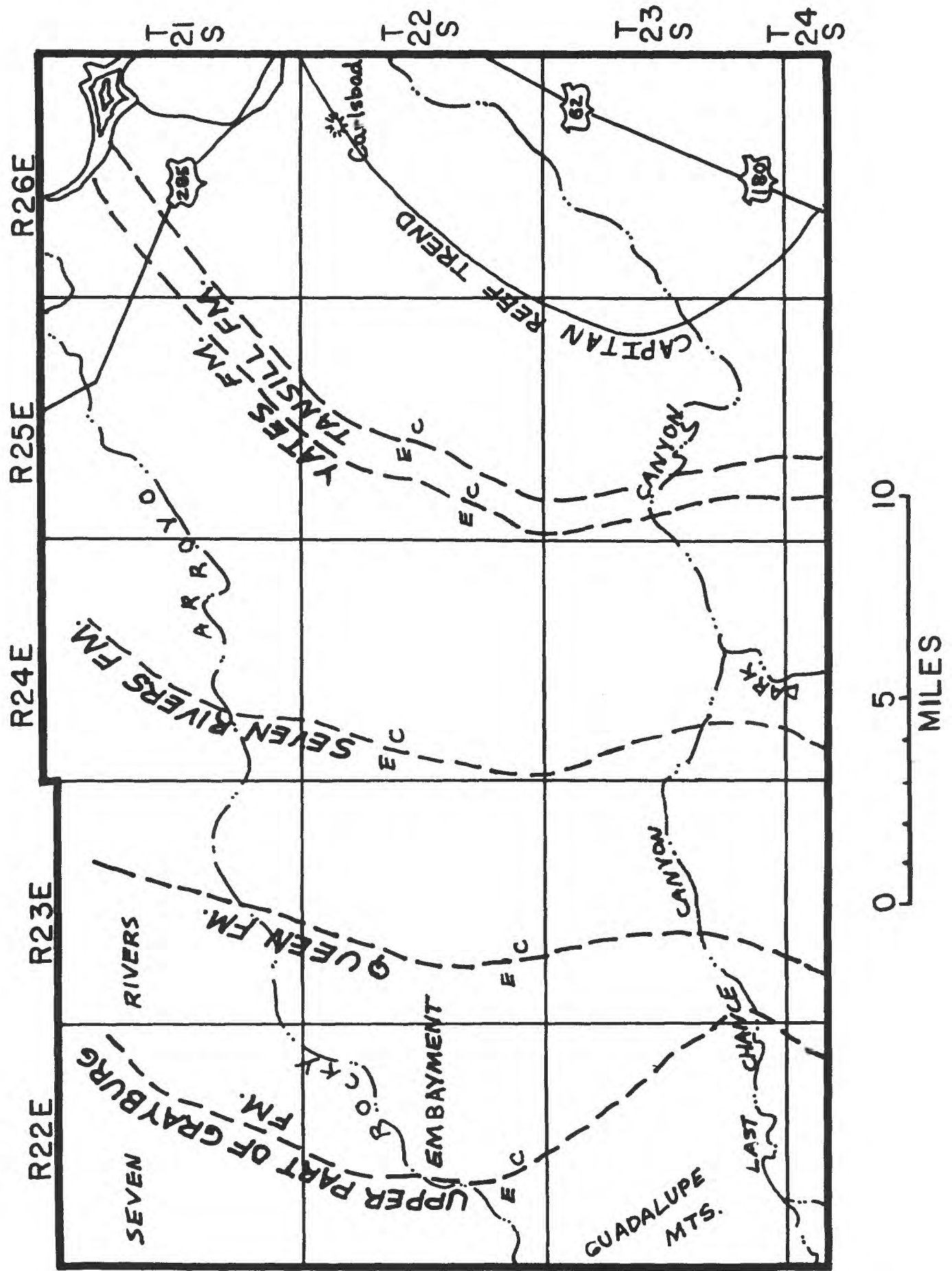

Figure 34.--Geographic location of the evaporite-carbonate transition in formations of the Artesia Group relative to the position of the uppermost Capitan reef facies. Adapted from bjorklund and Motts (1959) and Motts (1968). 

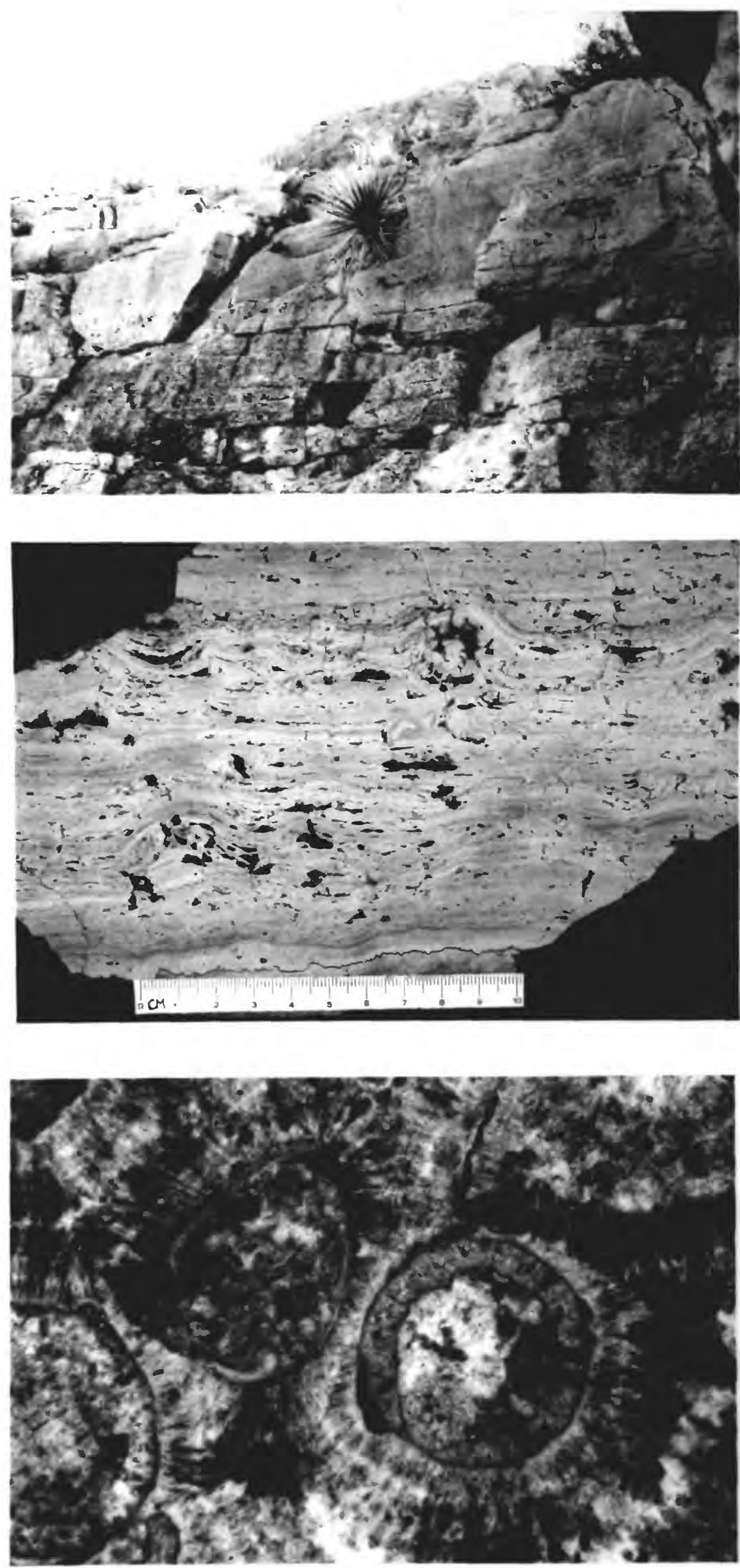

Figure 35.--Seaward-dipping low angle, cross-bedded grainstones in Tansill Formation at stop III-2 (Dark Canyon). These grainstones probably represent windward beaches on small islands. Cactus in center is approximately 2 feet high.

Figure 36.--Polished rock slab showing birdseye structures, fenestral porosity, and probable blue-green algal lamination of dolomitic mudstone in the Tansill Formation.

Figure 37.--Photomicrograph of green-algal grainstone showing coarse, radial, cloudy, fibrous, submarine cements (originally probably aragonite, now calcite). Long axis of photo equals $2.0 \mathrm{~mm}$. 

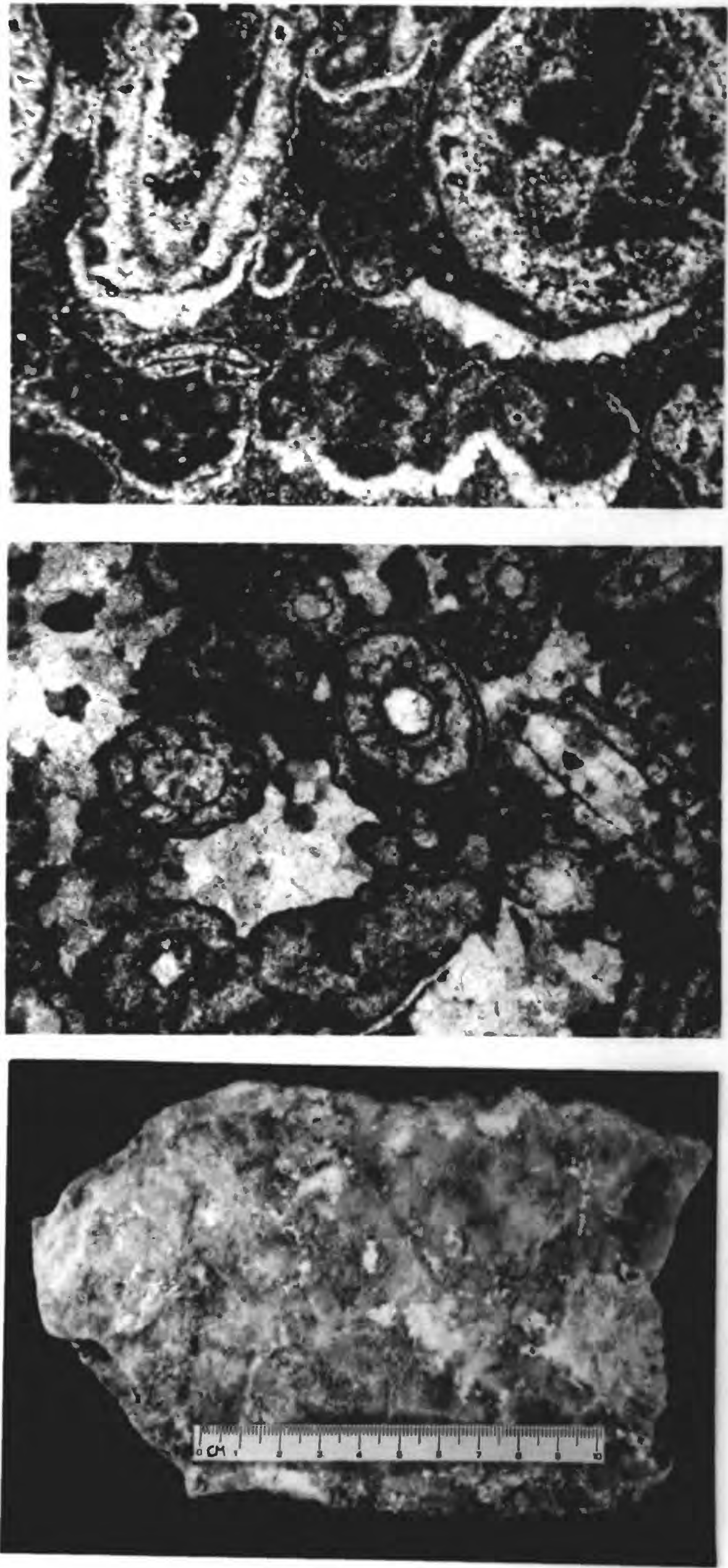

Figure 38.--Photomicrograph of green-algal grainstone showing microstalactitic or pendant calcite cement morphology indicative of cementation in a vadose (fresh water) environment. Long axis of photo equals $2.0 \mathrm{~mm}$.

Figure 39.--Photomicrograph of green-algal grainstone showing blocky calcite cement fabric indicative of cementation in a phreatic (fresh water) environment. Long axis of photo equals $2.5 \mathrm{~mm}$.

Figure 40.--Polished rock slab showing altered nodular or enterolithic fabric in back-reef bedded gypsum. 


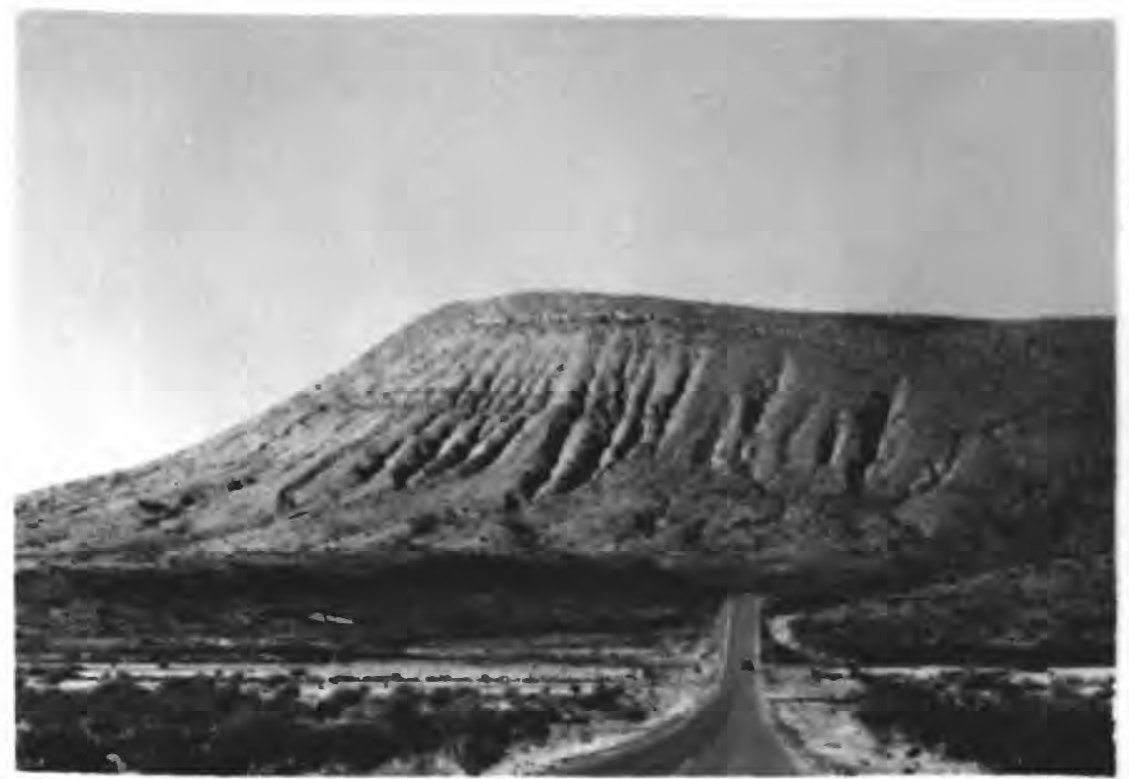

Figure 41.--Margin of the Seven Rivers Embayment along New Mexico Highway 137. Cliffs are capped by the resistant "Azotea Dolomite"; deeply gullied slopes are underlain by interbedded gypsum and red siltstone.

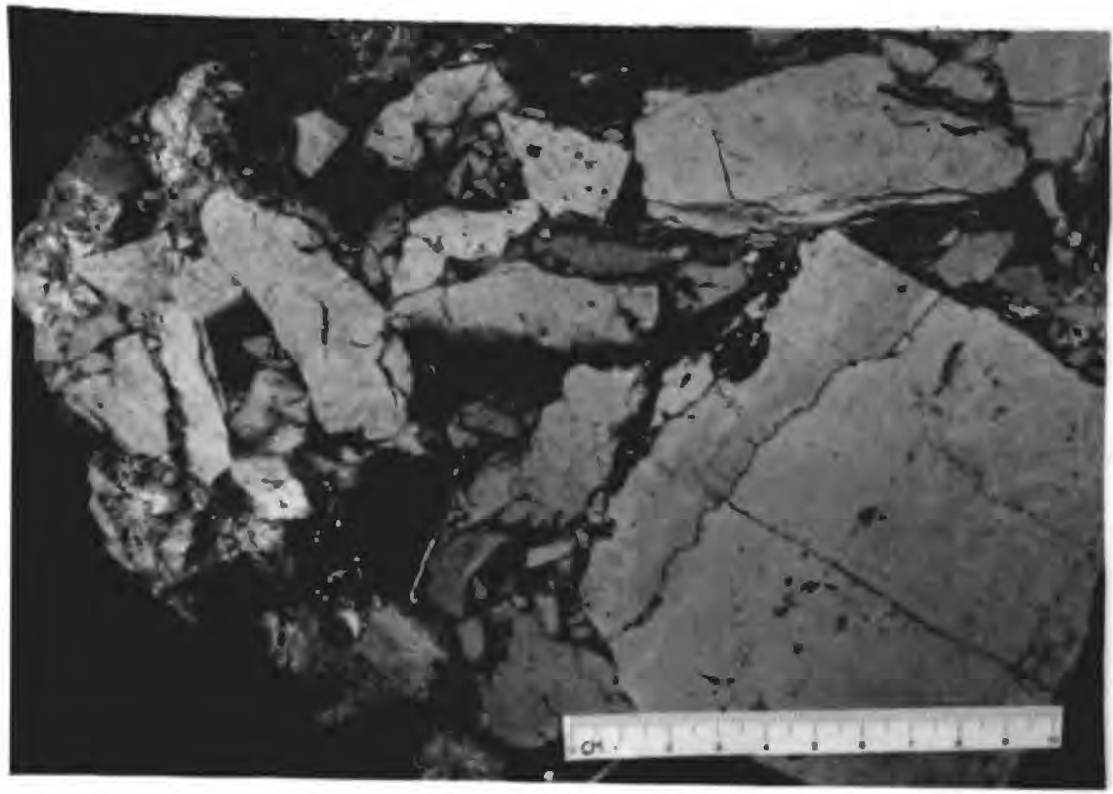

Figure 42.--Polished rock slab showing evaporite solution breccia from Rocky Arroyo. Note angular dolomite clasts and sparry calcite pore-lining cement.

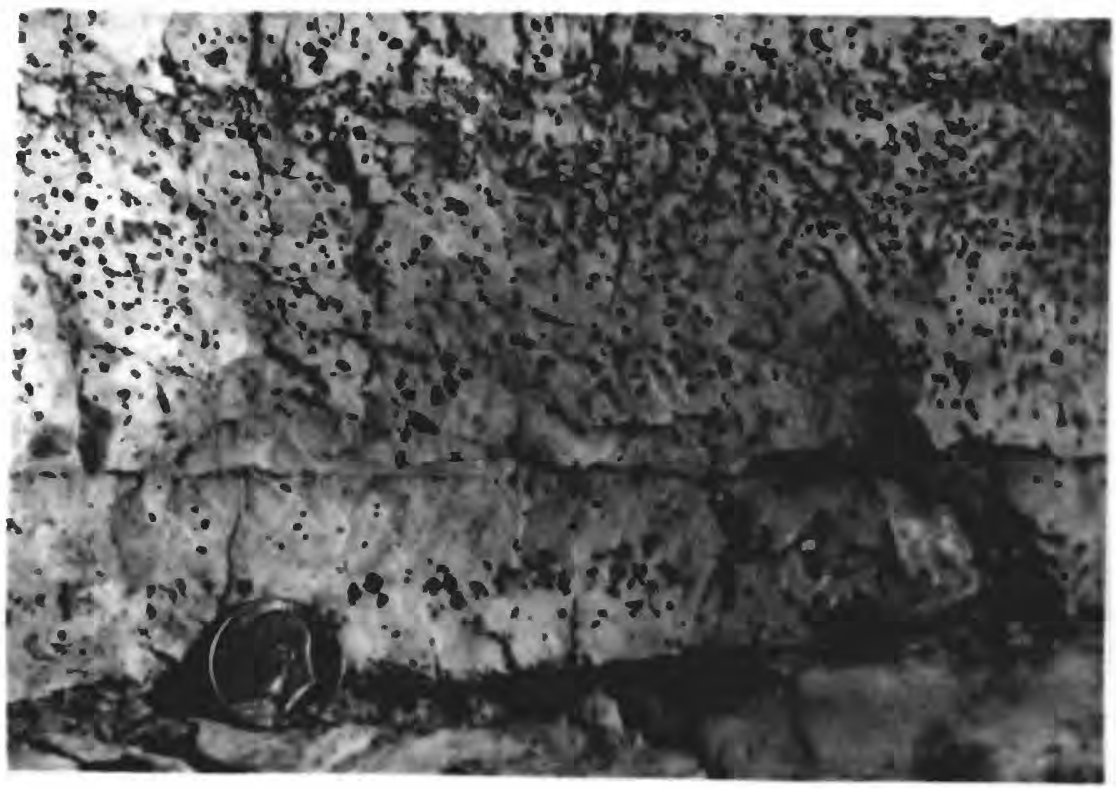

Figure 43.--Crystal casts produced by the selective removal of evaporite minerals from Seven Rivers dolomite beds. 
Table 1.--Correlations between faunal and sedimentary features and depositional environment (Data partially adapted from Newell and others (1953) and Schmidt (1977))

$[\mathrm{C}=$ common; $\mathrm{P}=$ present; $\mathrm{R}=$ rare; * indicates feature is generally or always detrital or allochthonous. Absence of any symbol indicates feature is absent or extremely rare]

\begin{tabular}{|c|c|c|c|c|c|c|c|}
\hline \multirow{3}{*}{ Feature } & \multicolumn{6}{|c|}{$\begin{array}{c}\text { Relative abundance in stratigraphic unit } \\
\text { and/or environment }\end{array}$} & \multirow{3}{*}{$\begin{array}{l}\text { Bel1 } \\
\text { Canyon Fm. } \\
\text { Basin }\end{array}$} \\
\hline & \multicolumn{3}{|c|}{ Artesia Group } & \multicolumn{3}{|c|}{ Capitan Ls. } & \\
\hline & $\begin{array}{l}\text { Sabkha- } \\
\text { lagoon }\end{array}$ & $\begin{array}{l}\text { Pisolite } \\
\text { facies }\end{array}$ & $\begin{array}{l}\text { Near } \\
\text { back-reef }\end{array}$ & \multirow[t]{2}{*}{$\begin{array}{l}\text { Reef } \\
\text { crest }\end{array}$} & \multicolumn{2}{|c|}{$\begin{array}{l}\text { Forereef } \\
\text { Up. Lo. }\end{array}$} & \\
\hline \multicolumn{7}{|l|}{ Fauna: } & \\
\hline Ostracodes & $\mathrm{P}$ & $\mathrm{R}$ & $\mathrm{P}$ & $\mathrm{R}$ & $\mathrm{R}$ & $\mathrm{R}$ & $\mathrm{R}$ \\
\hline Calcispheres & C & $\mathrm{p}$ & $\mathrm{P}$ & & & & $\mathrm{P}$ \\
\hline Stromatolites & $\mathrm{P}$ & $P$ & $\mathrm{R}$ & $\mathrm{P}$ & $\mathrm{P}$ & & \\
\hline Dasyclad algae & & $\mathrm{R}$ & $\mathrm{C}$ & $\mathrm{R}$ & $\mathrm{R} *$ & $\mathrm{R} *$ & \\
\hline Fusulinids & & $\mathrm{P}$ & $\mathrm{C}$ & $\mathbf{R}$ & $\mathrm{R}^{*}$ & $\mathrm{R}^{*}$ & $P$ \\
\hline \multicolumn{8}{|l|}{ Other } \\
\hline Foraminifera & $\mathrm{P}$ & $\mathbf{R}$ & $\mathrm{P}$ & $\mathrm{P}$ & $\mathrm{R}$ & $\mathrm{R}$ & $\mathrm{R}$ \\
\hline Gastropods & & $\mathrm{R}$ & $\mathrm{C}$ & $P$ & $\mathrm{R}$ & & \\
\hline Pelecypods & & $\mathrm{R}$ & $\mathrm{C}$ & $\mathrm{P}$ & & & \\
\hline Red algae & & & $\mathrm{P}$ & $\mathrm{C}$ & $\mathrm{P}$ & & \\
\hline Echinoderms & & $\mathbf{R}$ & $\mathrm{P}$ & $\mathrm{P}$ & $\mathrm{P}$ & $\mathbf{p}$ & $\mathrm{R}$ \\
\hline Brachiopods & & & $\mathrm{P}$ & $\mathrm{P}$ & $\mathrm{P}$ & $\mathrm{c}$ & $\mathrm{R}$ \\
\hline \multicolumn{8}{|l|}{ Calcareous } \\
\hline sponges & & & & $\mathrm{C}$ & C & $\mathrm{P} *$ & \\
\hline Hydrozoans & & & & $\mathrm{C}$ & C & & \\
\hline Tubiphytes & & & & $\mathrm{C}$ & $\mathrm{C}$ & & \\
\hline Bryozoans & & & & $\mathrm{C}$ & $\mathrm{C}$ & $\mathrm{p}$ & \\
\hline Ammonoids & & & $\mathrm{R}$ & $\mathrm{R}$ & $\mathrm{R}$ & $\mathrm{R}$ & $\mathrm{R}$ \\
\hline \multicolumn{8}{|l|}{ Siliceous } \\
\hline sponge spicul & les & & & & & $\mathrm{C}$ & $\mathrm{P}$ \\
\hline Conodonts & & & & & & $\mathrm{R}$ & $\mathrm{p}$ \\
\hline Radiolarians & & & & & & & $\mathrm{P}$ \\
\hline Fish & & & & & & & $\mathrm{R}$ \\
\hline \multicolumn{8}{|c|}{ Carbonate rock types: } \\
\hline Bounds tone & $P$ & & $\mathrm{R}$ & $\mathrm{C}$ & $\mathrm{C}$ & $\mathrm{P} *$ & \\
\hline Grainstone & & $\mathrm{C}$ & C & $P$ & $P$ & $\mathrm{P}$ & \\
\hline \multicolumn{8}{|l|}{ Packstone } \\
\hline Wackestone & $\mathrm{R}$ & $\mathrm{P}$ & $\mathrm{P}$ & $\mathrm{P}$ & $\mathrm{c}$ & $\mathrm{C}$ & $\mathrm{P}$ \\
\hline Mudstone & $\mathrm{C}$ & $\mathrm{P}$ & $\mathrm{P}$ & $\mathrm{R}$ & $\mathrm{R}$ & $\mathrm{P}$ & $\mathrm{C}$ \\
\hline \multicolumn{8}{|c|}{ Non-carbonate rock types: } \\
\hline Shale & & & & & & & C \\
\hline Siltstone & $\mathrm{C}$ & $\mathrm{P}$ & $\mathrm{R}$ & & & $\mathrm{P}$ & $\mathrm{C}$ \\
\hline Sandstone & $\mathrm{C}$ & $\mathrm{P}$ & $\mathrm{R}$ & & & $\mathrm{p}$ & $\mathrm{C}$ \\
\hline
\end{tabular}


Table 1.--Continued.

\begin{tabular}{|c|c|c|c|c|c|c|c|}
\hline \multirow[t]{2}{*}{ Feature } & \multicolumn{3}{|c|}{ Artesia Group } & \multicolumn{3}{|c|}{ Capitan Ls. } & \multirow{2}{*}{$\begin{array}{l}\text { Bell } \\
\text { Canyon Fm. } \\
\text { Basin }\end{array}$} \\
\hline & $\begin{array}{l}\text { Sabkha- } \\
\text { lagoon }\end{array}$ & $\begin{array}{l}\text { Pisolite } \\
\text { facies }\end{array}$ & \multirow[t]{2}{*}{$\begin{array}{l}\text { Near } \\
\text { back-reef }\end{array}$} & \multirow[t]{2}{*}{$\begin{array}{l}\text { Reef } \\
\text { crest }\end{array}$} & \multicolumn{2}{|c|}{$\begin{array}{l}\text { Forereef } \\
\text { Up. Lo. }\end{array}$} & \\
\hline $\begin{array}{l}\text { Organic carbon- } \\
\text { rich units }\end{array}$ & $\mathrm{R}$ & & & & & $\mathrm{P}$ & $\mathrm{C}$ \\
\hline \multicolumn{8}{|l|}{ Grain types: } \\
\hline Skeletal & $\mathrm{R}$ & $\mathrm{P}$ & C & C & C & $\mathrm{P}$ & $P$ \\
\hline Pelletal & C & $\mathbf{R}$ & $\mathrm{P}$ & $\mathrm{P}$ & $\mathrm{P}$ & $\mathrm{R}$ & \\
\hline Pisolitic/oolitic & $\mathrm{R}$ & $\mathrm{C}$ & $\mathrm{P}$ & & & & \\
\hline Intraclastic & $\mathrm{R}$ & $P$ & $P$ & C & C & $\mathrm{P}$ & $\mathrm{R}$ \\
\hline \multicolumn{8}{|l|}{$\begin{array}{l}\text { Sedimentary } \\
\text { structures: }\end{array}$} \\
\hline Lamination & $\mathrm{C}$ & $\mathrm{R}$ & $\mathrm{R}$ & & & $\mathrm{P}$ & C \\
\hline Bedding & C & $\mathrm{C}$ & $\mathrm{P}$ & & $\mathrm{R}$ & $\mathrm{P}$ & $\mathrm{C}$ \\
\hline $\begin{array}{l}\text { Synsedimentary } \\
\text { fractures }\end{array}$ & & & $\mathrm{P}$ & $\mathrm{C}$ & $\mathrm{C}$ & & \\
\hline Channels & & & $\mathrm{P}$ & $?$ & C & $\mathrm{P}$ & $\mathrm{C}$ \\
\hline Cross-bedding & & $\mathrm{R}$ & $\mathrm{P}$ & & $\mathrm{R}$ & $\mathrm{P}$ & $P$ \\
\hline Graded bedding & & & $\mathrm{R}$ & & $\mathrm{R}$ & $\mathrm{P}$ & $\mathrm{P}$ \\
\hline Breccias & $\mathrm{C}$ & & & & C & $\mathrm{P}$ & $\mathrm{R}$ \\
\hline Tepee structures & & C & & & & & \\
\hline \multicolumn{8}{|c|}{ Diagenetic features: } \\
\hline Dolomitization & $\mathrm{C}$ & $\mathrm{C}$ & $P$ & & $\mathrm{P}$ & $\mathbf{P}$ & \\
\hline Chertification & & & & & $P$ & $\mathrm{C}$ & $\mathrm{C}$ \\
\hline Evaporite nodules & $\mathrm{C}$ & $\mathrm{R}$ & & & & & \\
\hline $\begin{array}{l}\text { Freshwater } \\
\text { cements }\end{array}$ & $\mathrm{R}$ & C & $\mathrm{P}$ & & & & \\
\hline Marine cements & & & $P$ & $\mathrm{C}$ & $\mathrm{C}$ & $\mathbf{P}$ & \\
\hline $\begin{array}{l}\text { Primary porosity } \\
\text { Secondary }\end{array}$ & $\mathrm{P}$ & $\mathbf{R}$ & $\mathrm{P}$ & & & & $\mathrm{C}$ \\
\hline porosity & C & $\mathrm{R}$ & C & $\mathrm{R}$ & & & \\
\hline
\end{tabular}




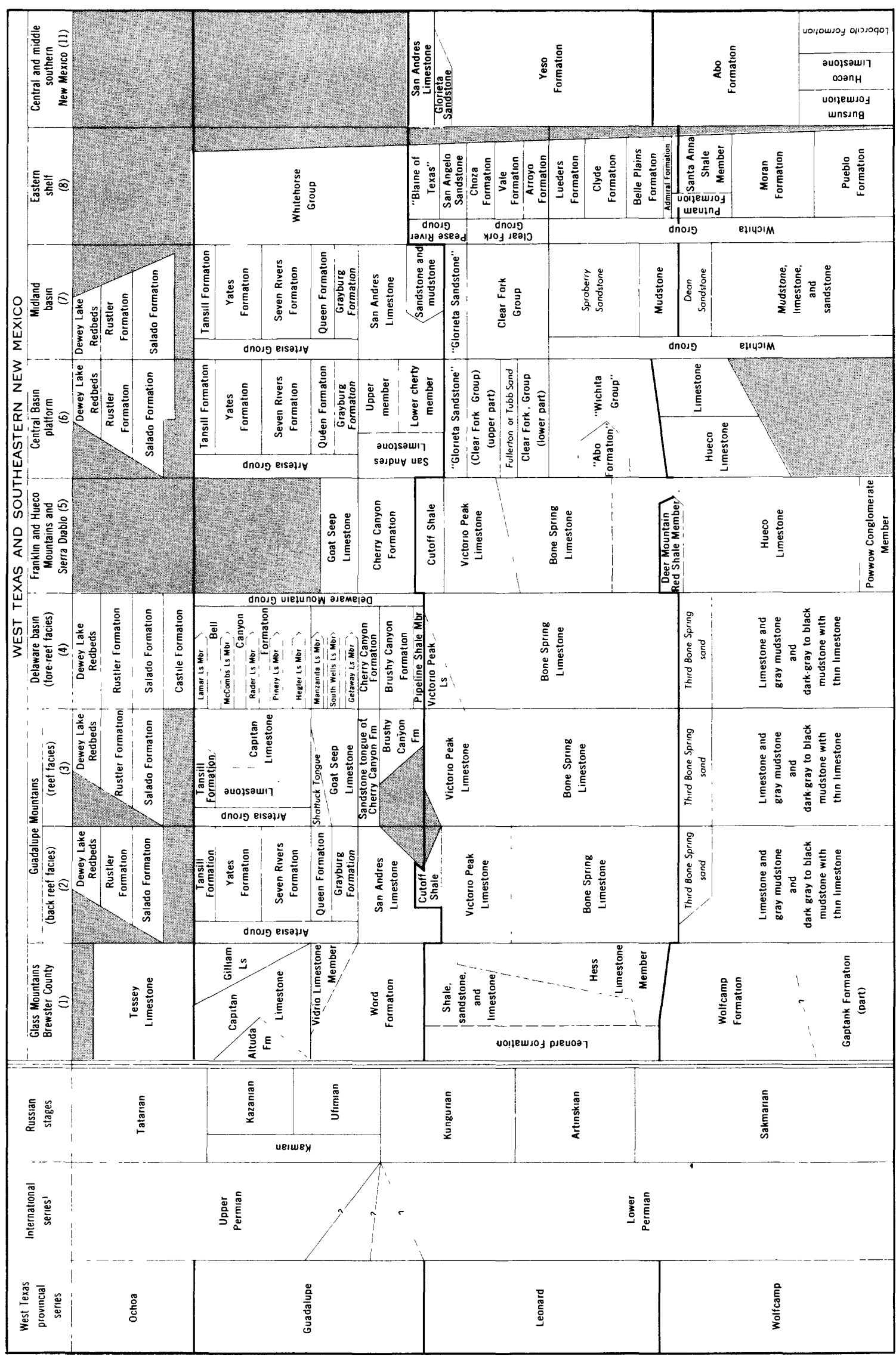

تี

ت

赵

E-

D

.

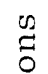

只

怘

(ن)

过

잉

Eี

出.

क 10

मत है

$\Xi$

تิ

嘼

\&

出

تص

준

氙.

0

$1 x$

i

$\underset{\substack{0 \\ 0-1}}{\infty}$ 


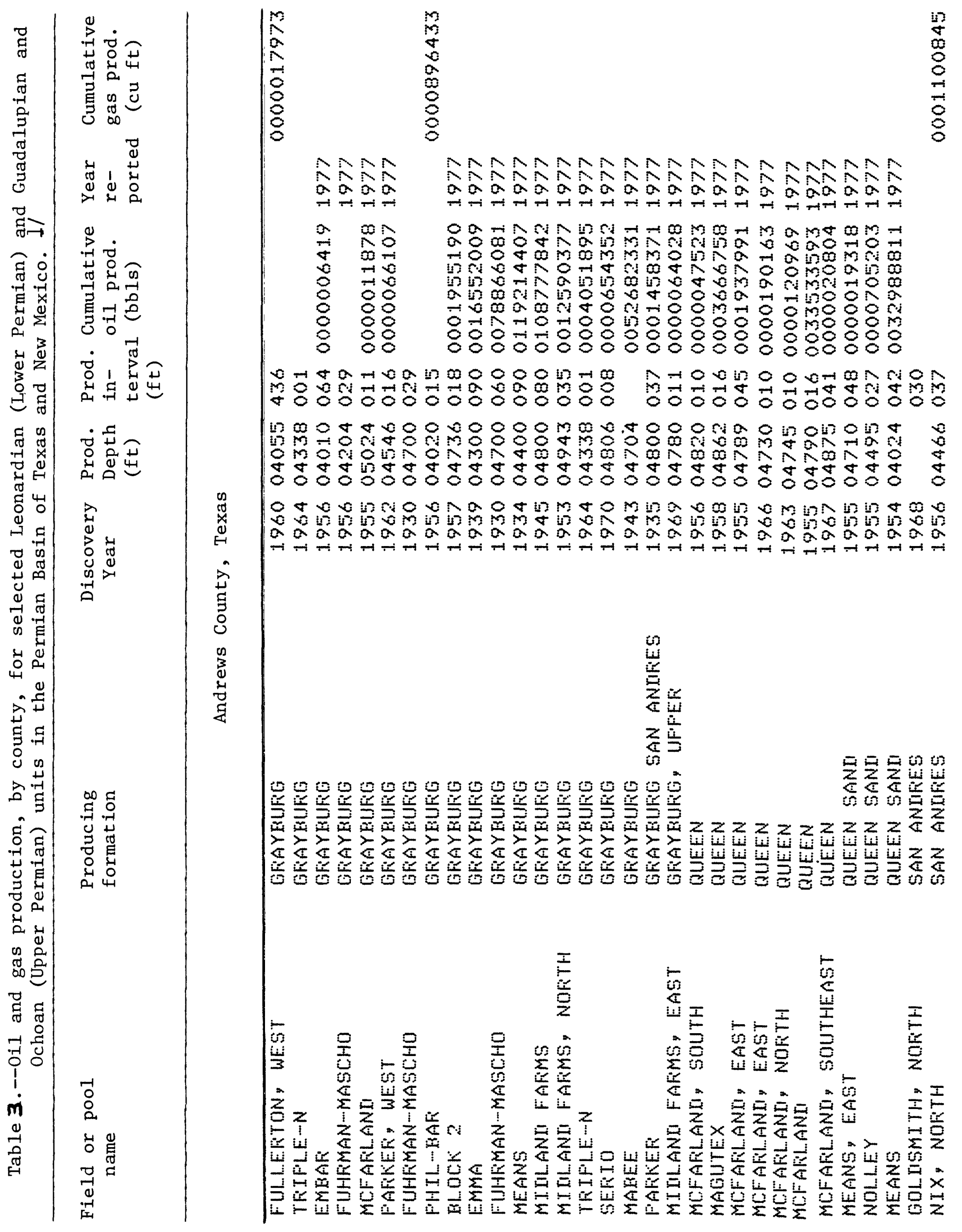


8

WNANANANAN 2. 202020000

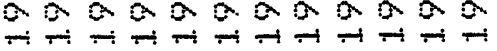

NNNNNNNNR ANANANRNAN

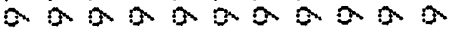

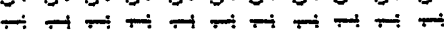
mámorimom $m \& 020000200$ तम mat 0 ino 0 - कrion iि $\rightarrow 00001008$ 58805807888 8888888888: in in वक्ठष trmo

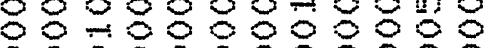

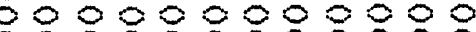

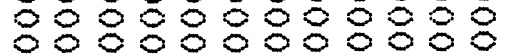

Nom

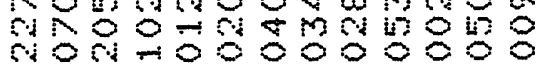

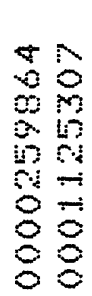

No

22

$\div \div$

$\operatorname{los}$

$\rightarrow 0$

8

-iㅇㅇㅇ

88

$\underset{8}{0} \underset{0}{0}$

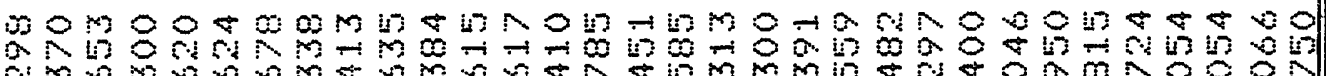

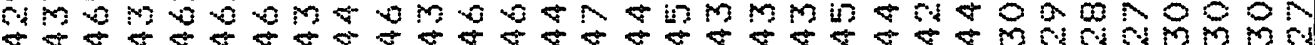
\$:

$\because 4 \mathrm{man}$ -

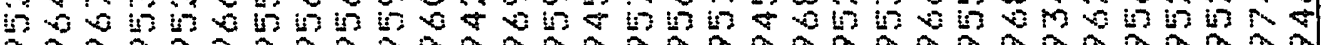

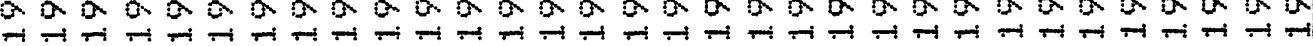

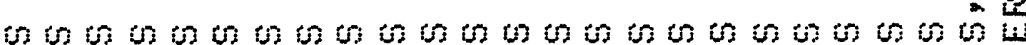

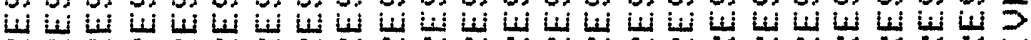

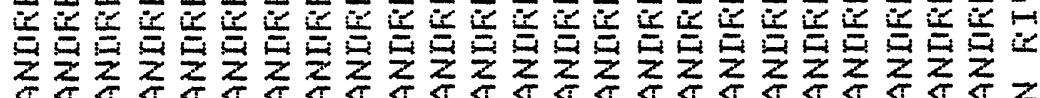

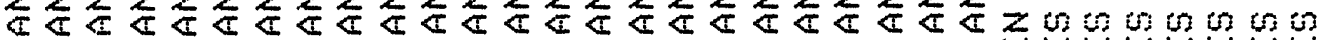

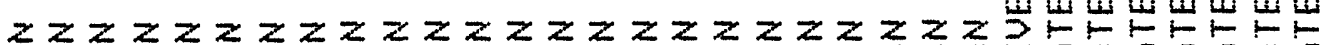

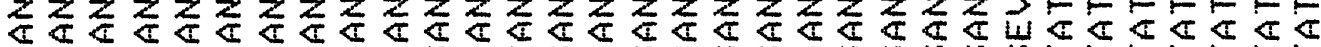
की

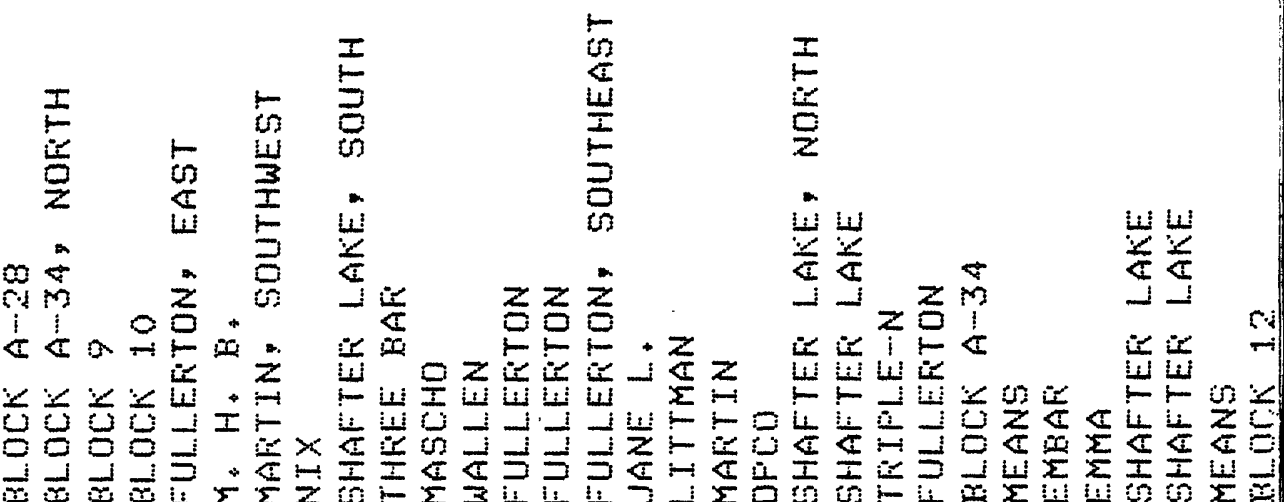

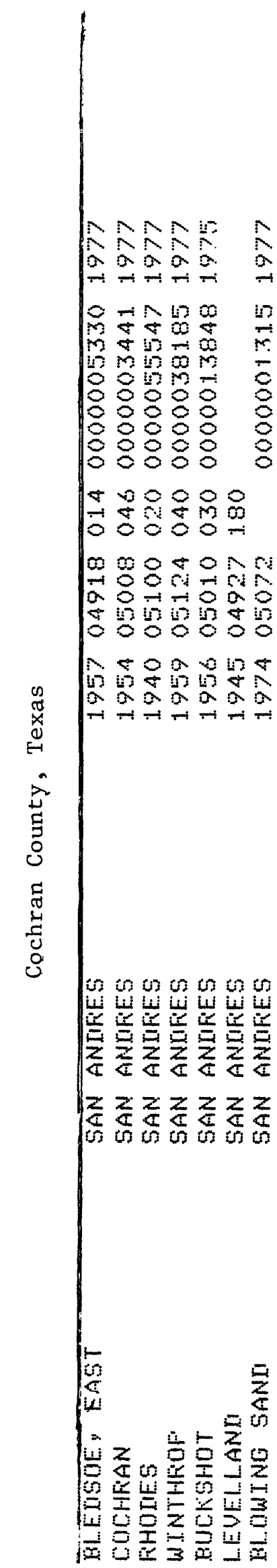




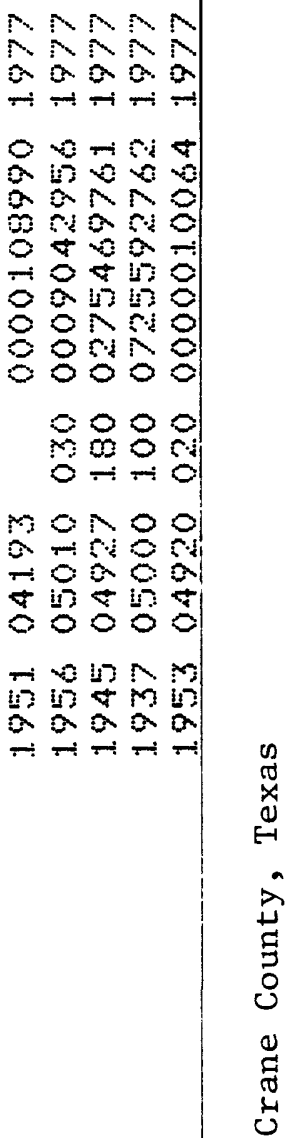

in 6 in the

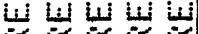
는 灵要烈

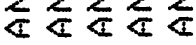

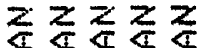

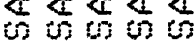

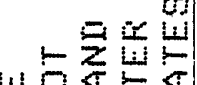
ZLit $4 \div \div \div$ $60=\frac{1}{0}$ 는 U?

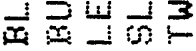

0
0
0
0
8
8

NN NSNR कर 202020

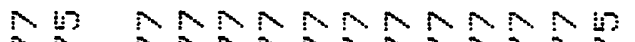

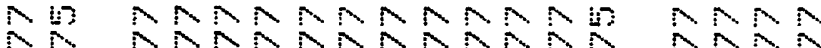

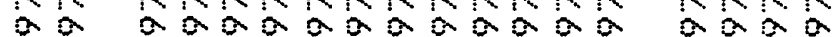

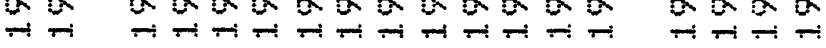
and in 00000 2 in

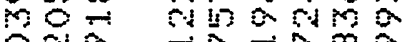
$800 \%$ $80 m$ + $m=\infty \omega$ ठㅇㅇ 886 की

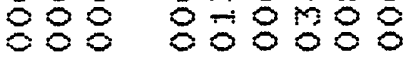

0
48
8
68
6 $0 N \infty+i n a \alpha$ an man

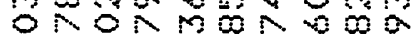
क巾

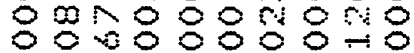

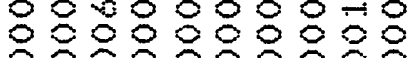

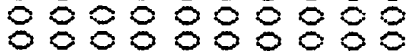

$\square$
$\square$
$n$
0
8
8
8

$00+\infty$

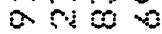
$\rightarrow$ in

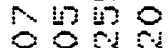
$8050 \%$

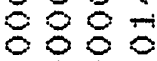
कo की

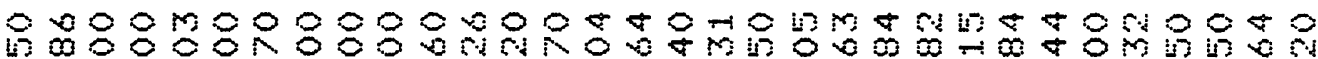
itm की की सेmmm

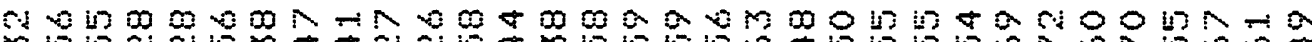

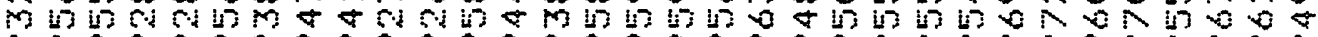

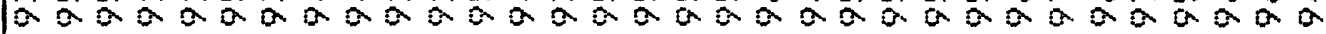
मب

$$
\begin{aligned}
& \sum_{i} \\
& \text { is } \frac{\pi}{5}
\end{aligned}
$$

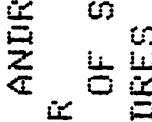

$$
\begin{aligned}
& \text { z讪永 } \\
& \text { 당ㄷㄴ } \\
& \text { (i) } \\
& 1-N \frac{2}{6}
\end{aligned}
$$

की

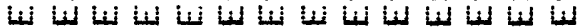

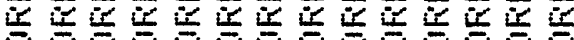

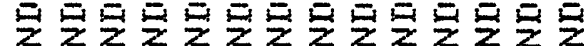

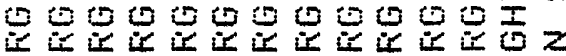

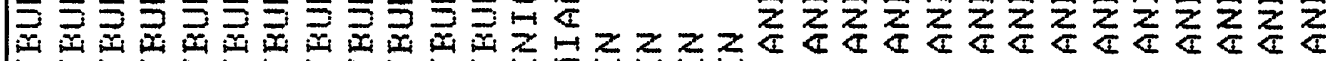

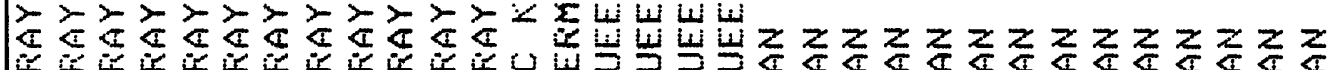

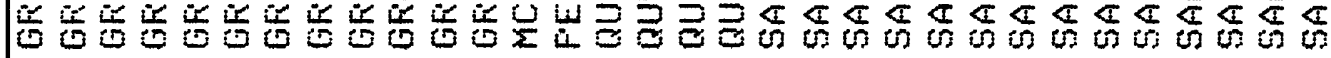

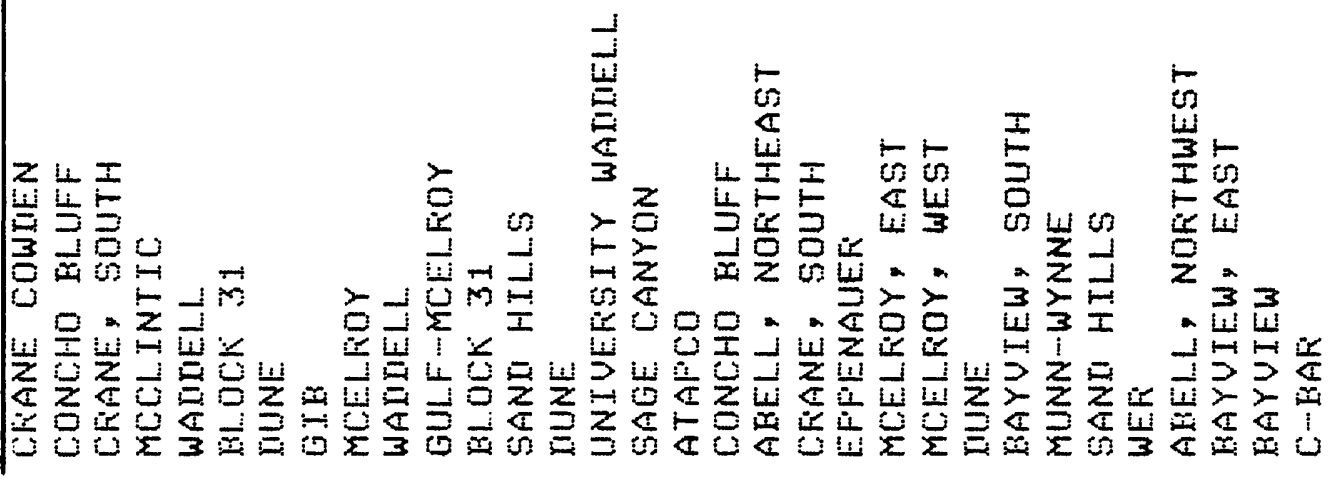




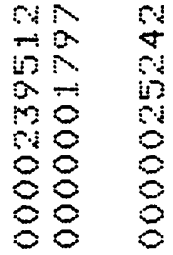

2
2
$=$
0
8
8
8

$m \infty 8$ O

혀 6

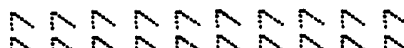
$\alpha a \alpha 2002020$

$+\infty \pi+280$ in +2 ing $688 \div 8888$ 8680880 0600600 पi $808-820$ $8 \%$

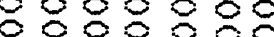
.000000
8
0
0
0
0

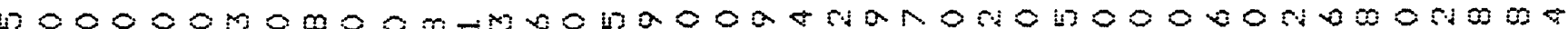

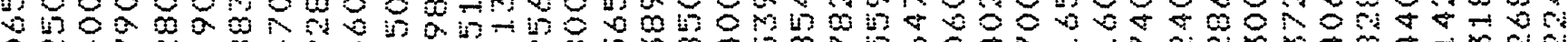
OR 60б0б

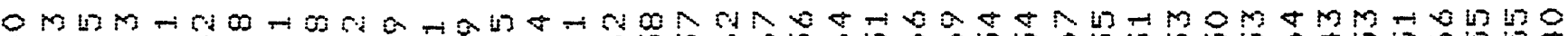

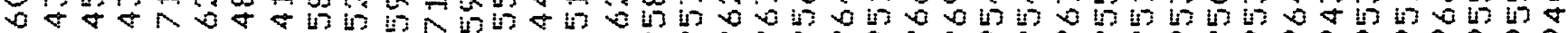

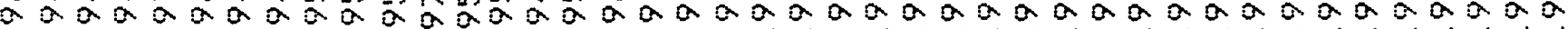

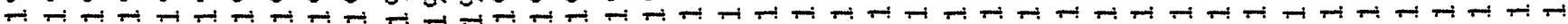

原

至

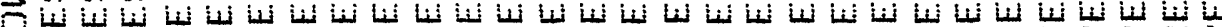

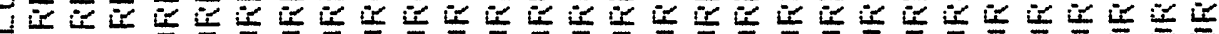

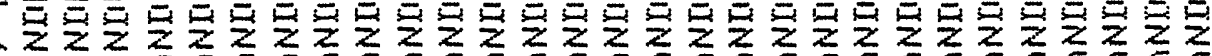

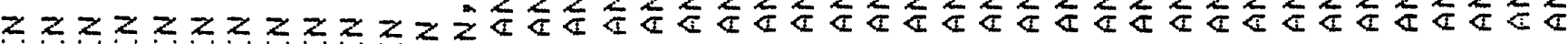

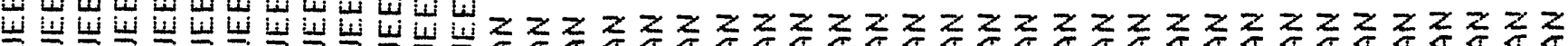

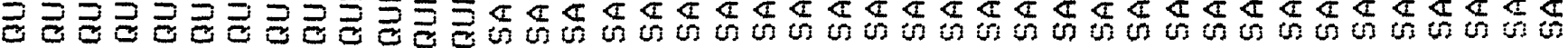

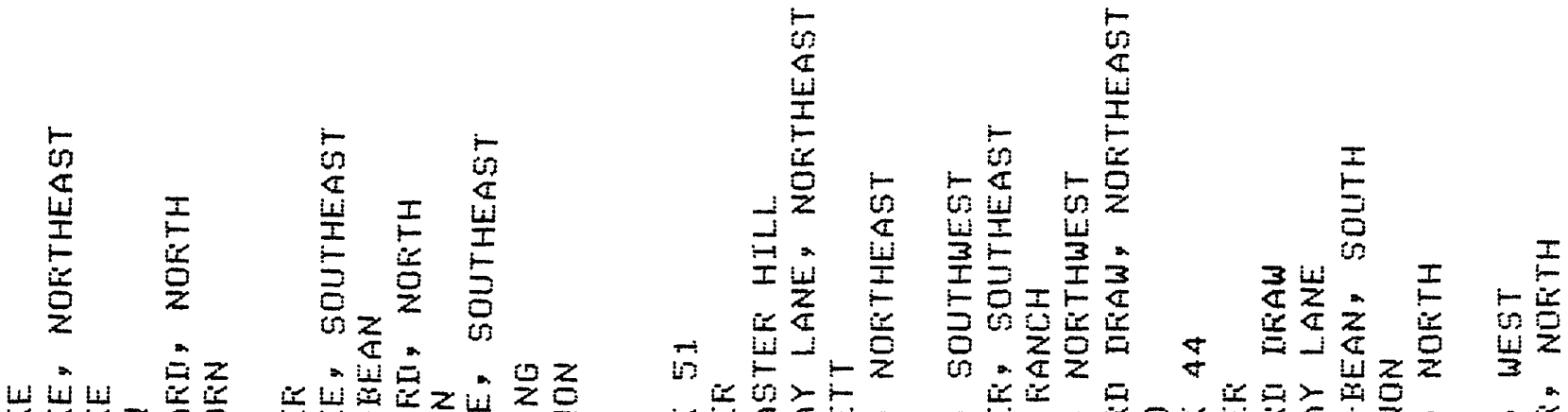

NANANANANAN

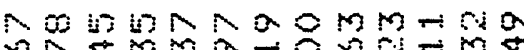
hon amanowan $000 \div+0,0$ $\rightarrow m 000 \%$

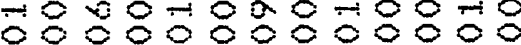

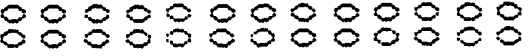

$0+4 \mathrm{i} ;+\cdots+00+80$ स0

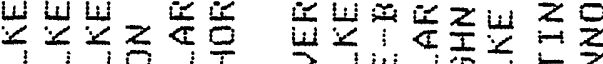

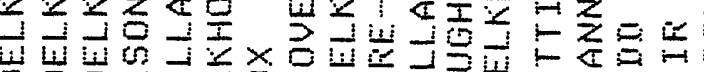

14 


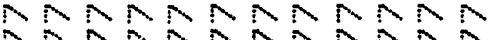
$\alpha 2 \alpha 2 \alpha \approx \alpha \approx \alpha \approx \alpha$

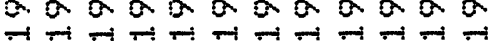

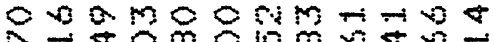

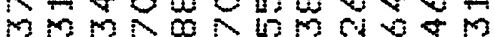
उ०क \& 0 \% ती नа山 000800008000 80888088888888

oOnOMMO n 00 एँ

mon ग05\% mmmam 0000000000000

MHOW

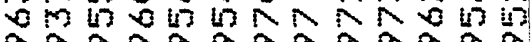
$\alpha \alpha 2 \alpha a \alpha a \alpha a \alpha a \alpha a$

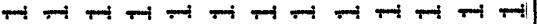

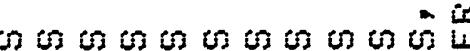

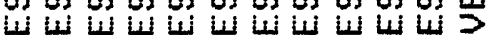

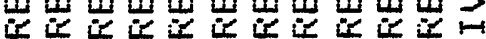

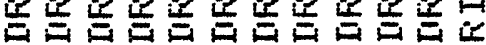

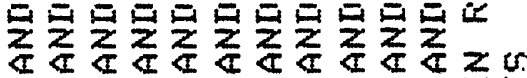
$z z z z z z z z z z z$ 岁

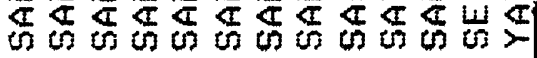

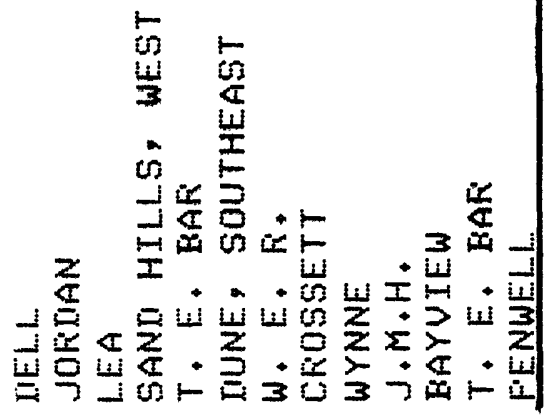

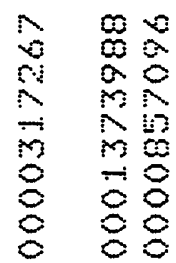

$m$
8
0
0
8
8

NMS

$2 \pi \Omega$

20202

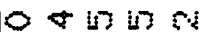

$0+m 2 a$

$0 \& m a$

a. $2=0$

ri $000 \div$

08080

88888

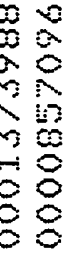

NNDNDSNDN

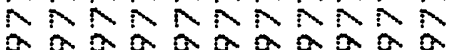

$2 \alpha 2 \alpha 2 \alpha 2 a 200$

2

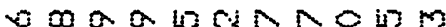

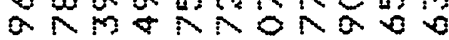

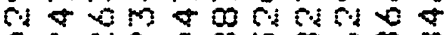

on 200 in

$8-1540 \% 5 \%$

○ी

$88088 \div 8808 \mathrm{0}$

88888588580

is तो तो 0

in

00 समे०

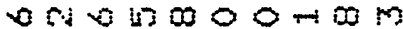

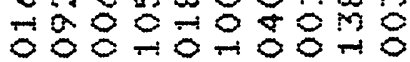
omotor

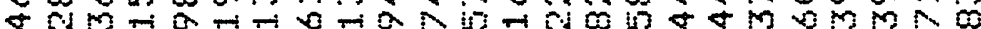

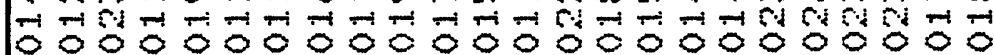

-iamo has (i) 0 is

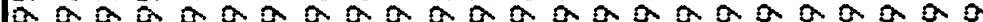

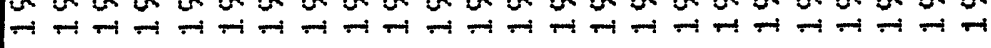

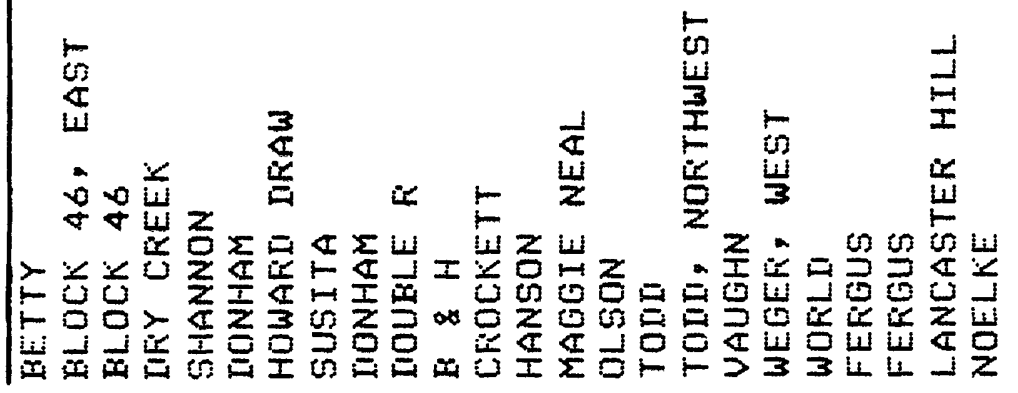




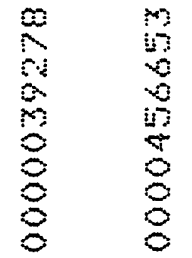

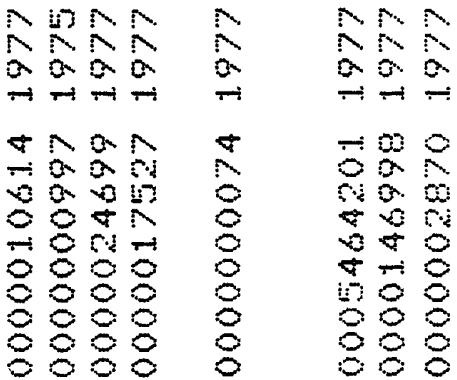

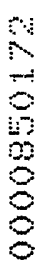

S

20

0

30

?

88

88 0. $0 \div$

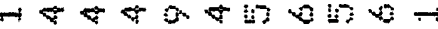

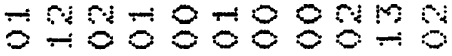

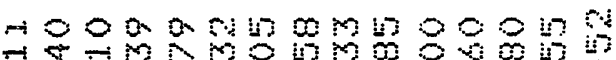

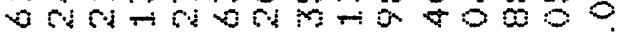

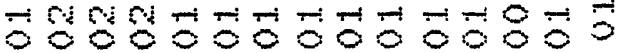

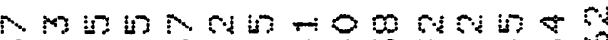

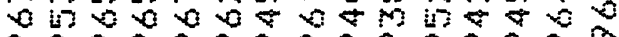
a 20000000000000

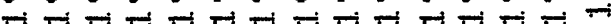

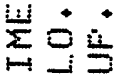

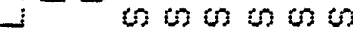

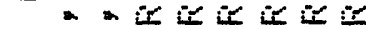

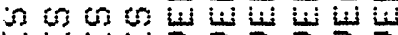

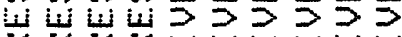

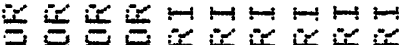

$\exists \equiv \Xi$

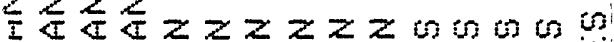

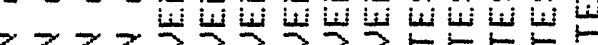

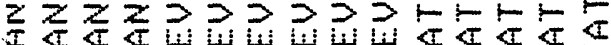

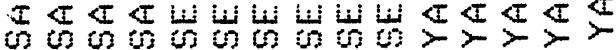

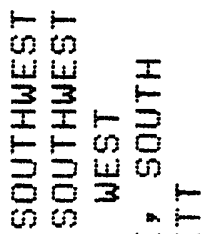

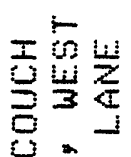

is $z$

$6 \div$

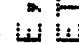

RNAN RNANANR a 2020202 $\rightarrow-\rightarrow+4$ नक

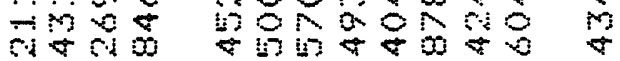

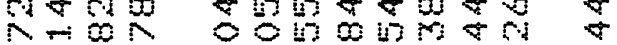

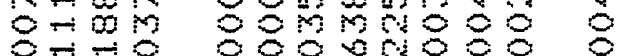

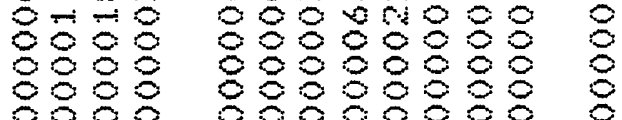

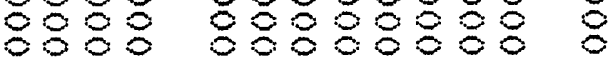
जo सम monmeष

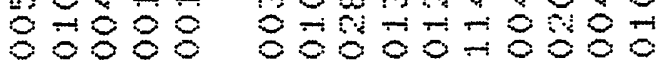

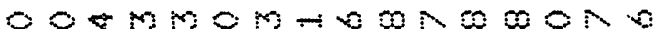

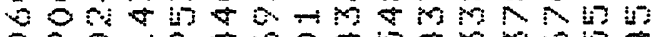
obtatootiammont

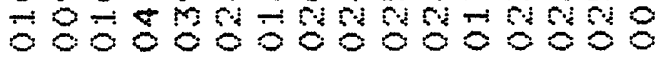
- OmaOnmañoma

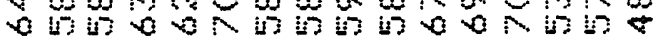
a a a a a a a a a a a a

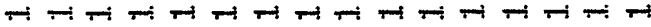

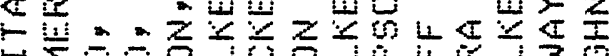

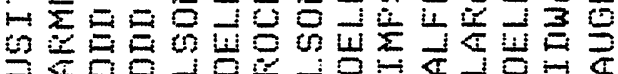

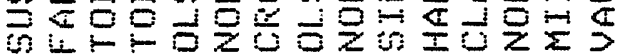

\section{른}

is

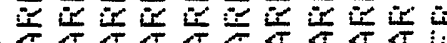

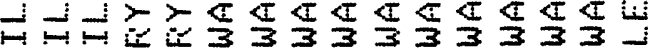

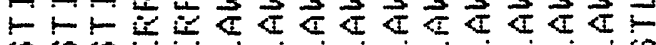

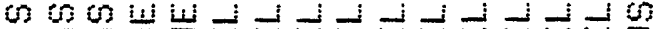

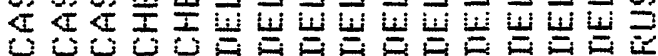

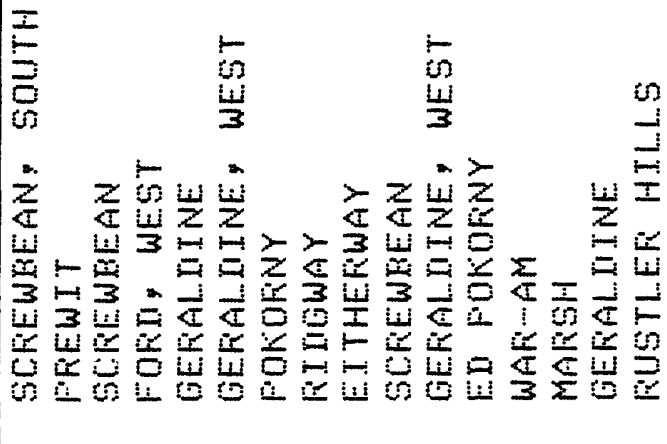


NNSN $N N N N M N$ 2020200 $\rightarrow-\rightarrow+\rightarrow+$

1) 00 ल का

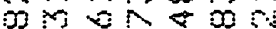

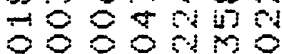
$6808 \div-5$ 200000

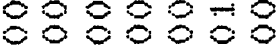

$\stackrel{5}{8}$

is 0

8 कण

$\therefore 0=1: 080$

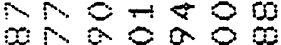
$m+12 \& 178$ 6000000

$m \& \&+\infty$ $\begin{array}{llll}42 & 2 & 2 & 2\end{array}$

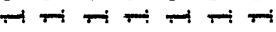

on on on on

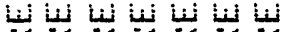

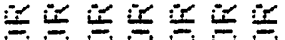

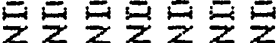

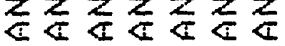

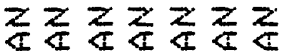

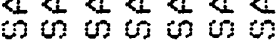

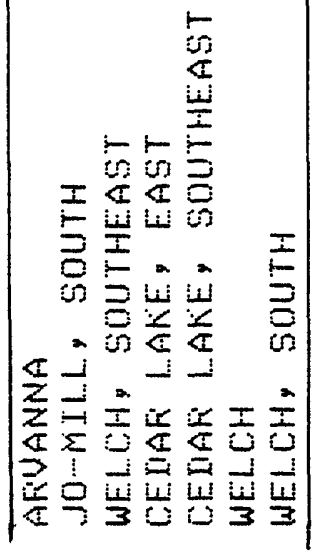

7
0
0
0
8
8

$\hat{N} \hat{N} \hat{R}$ 200202 $\rightarrow--\rightarrow-i$

$00 m-4$ और $a-a \operatorname{cic} 0$ -am-ris 80 is ㅇㅇㅇㅇㅇㅇ 685888

NRRNRNR $\alpha 2 \alpha \alpha 2 \alpha 2 \alpha 0$ $-\rightarrow+-\rightarrow-1-1$

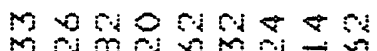
mos का का $\rightarrow$ तon स 2 in 0 का क 0 r. 00000

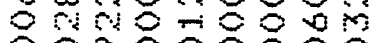

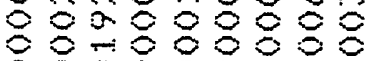

NANNAN NANANANAR 2002020002

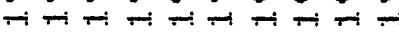

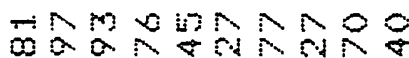
MNO \& 606ㅁ

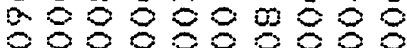
-006008\%08

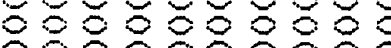

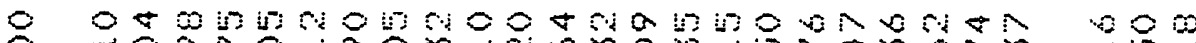

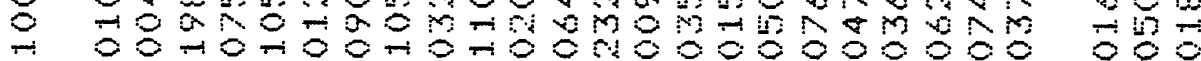

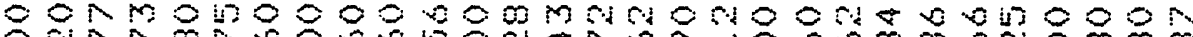

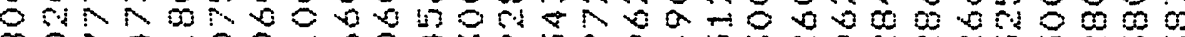
की m.

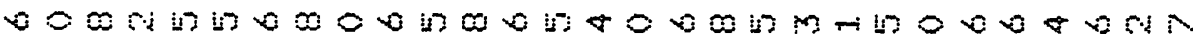
C. तो

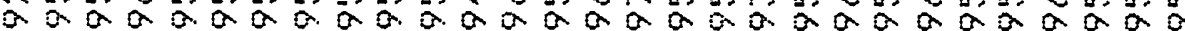

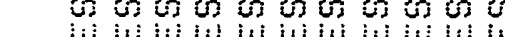

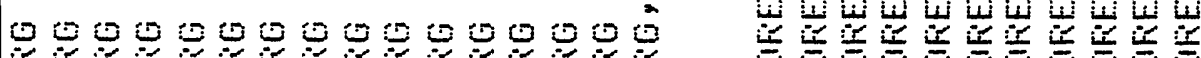

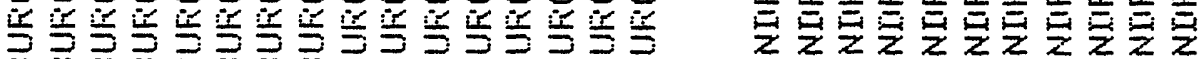

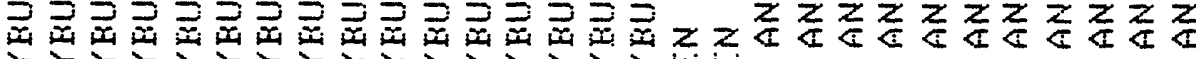

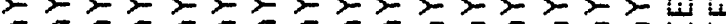

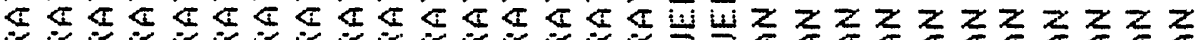

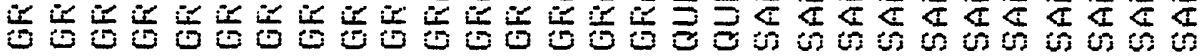

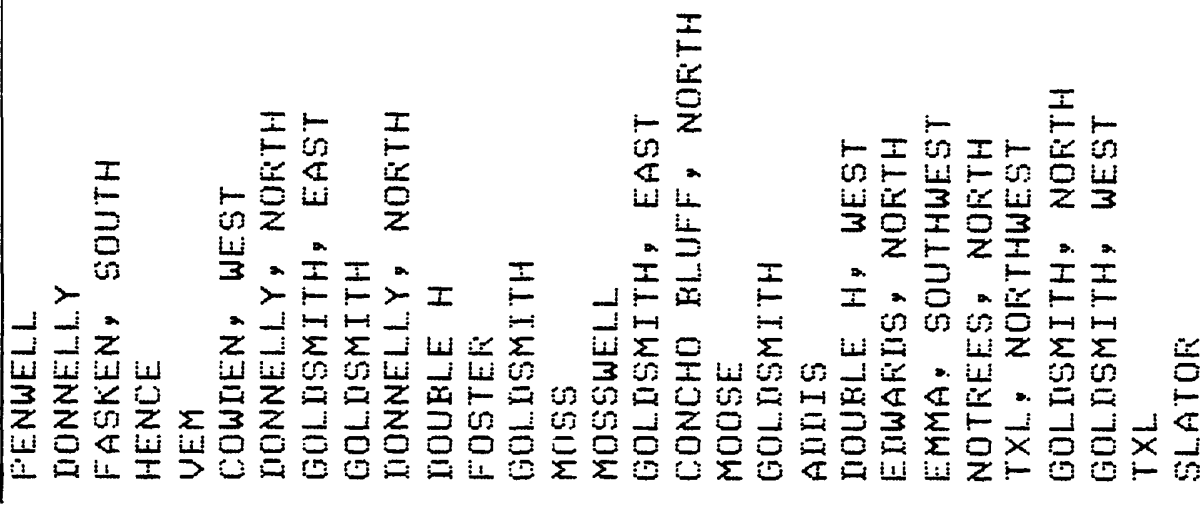


NMNANNANRNRN MNANANRNANMN

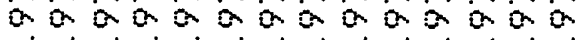
नi $\rightarrow$ -

न10000\% तथ

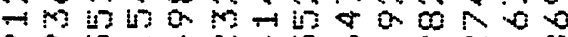

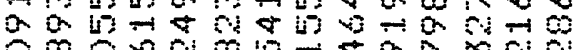
$0 m=0$ c. $\infty$ m 80 पर

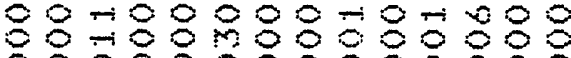
50000080068568

mino00+0 000000

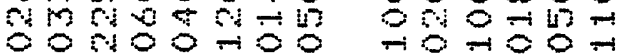

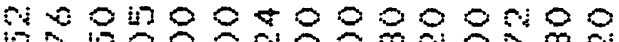
malomenom

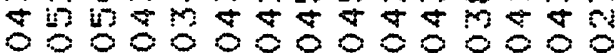
म

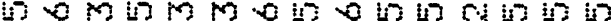
a 2020020000200

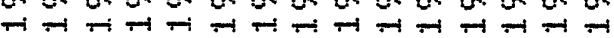

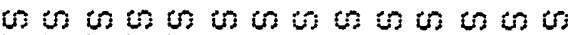

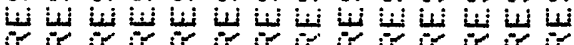

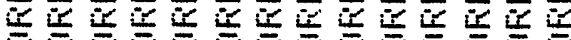

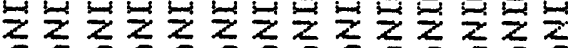

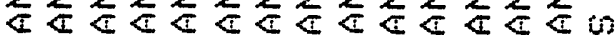

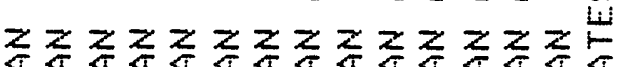

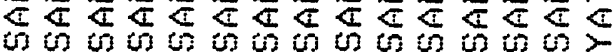

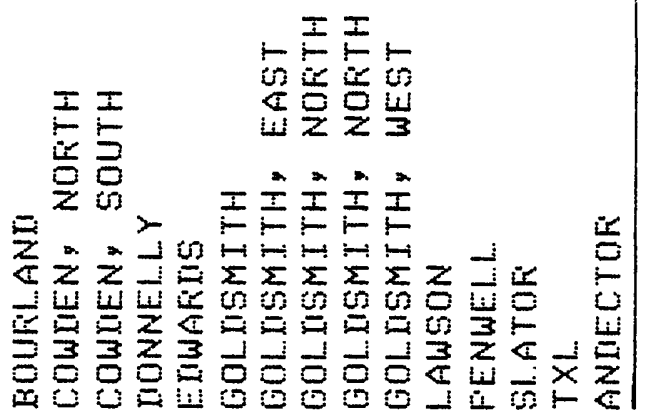

-4
0
0
8
8
8

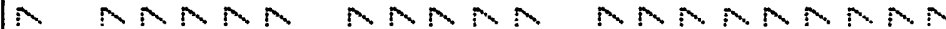
NNRNA NRNAN NRNRNRNRS

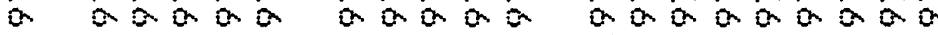
$\rightarrow$ Tान

$\leftarrow \rightarrow m \infty 00$

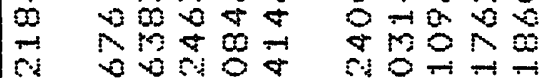
8 8퓨 08080 828850 888088 8888 88888

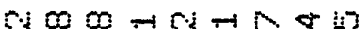
on 150 क Tho- $1000 \%$ in कNin

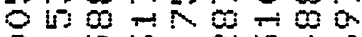
$8-15 m-10$ in 8008008 80080808 808088088

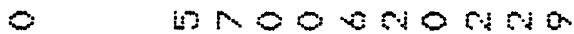

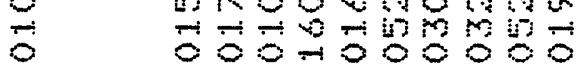
0 mo

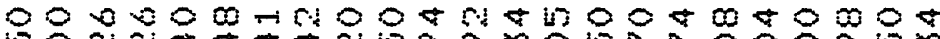
iन 0 \%

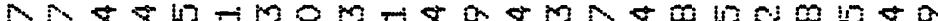

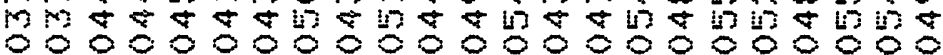

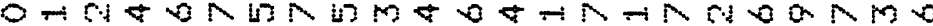

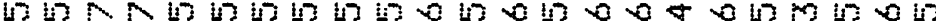

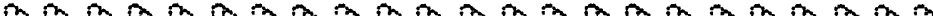

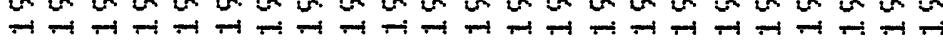

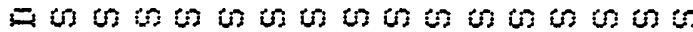

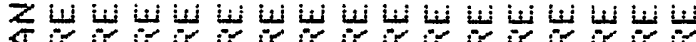

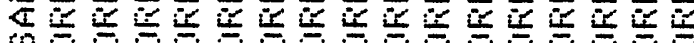

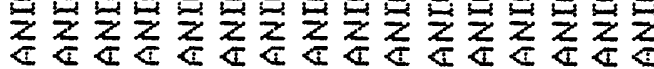

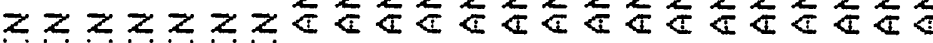

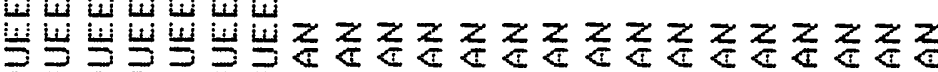

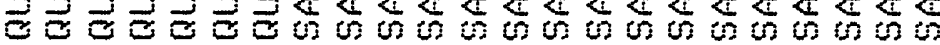

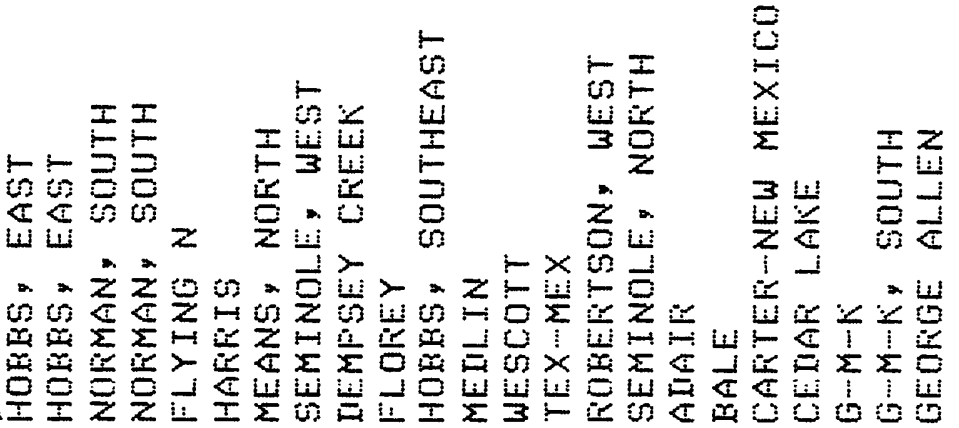




\begin{tabular}{|c|c|c|}
\hline $\begin{array}{l}50 \\
80 \\
08 \\
08 \\
88 \\
88 \\
88 \\
88\end{array}$ & $\begin{array}{l}80 \\
89 \\
80 \\
00 \\
94 \\
88 \\
80\end{array}$ & 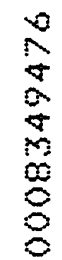 \\
\hline
\end{tabular}

NANANANANANANANAN NANANANANANANANAN

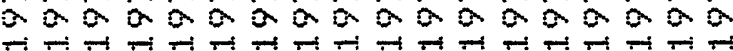
boم

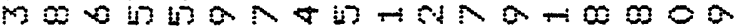

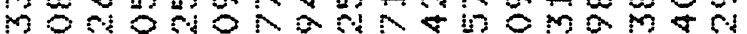

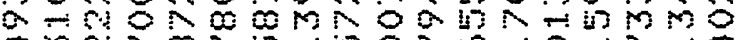

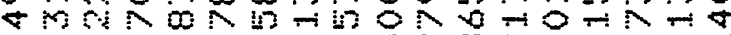

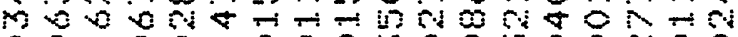

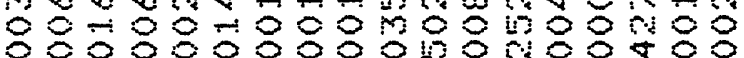
$88888888818 \% 88588$

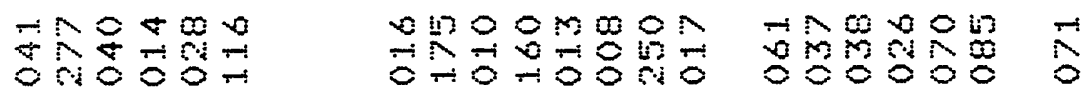

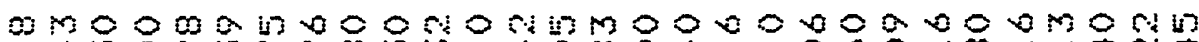

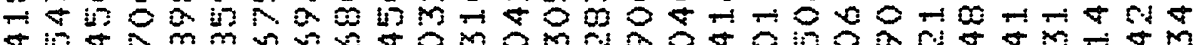
$+4+\infty$ 50050500305000005000000000000

13 और

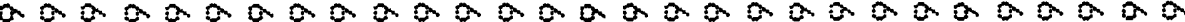

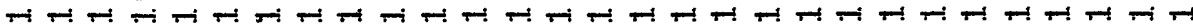

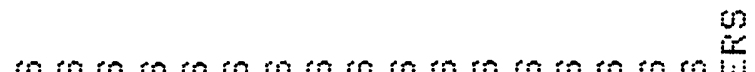

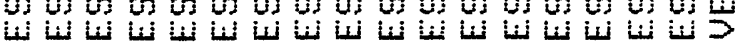

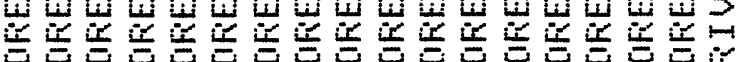

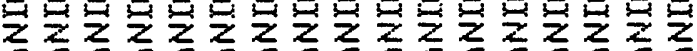

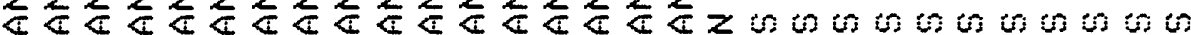

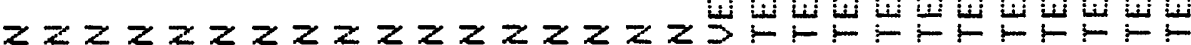

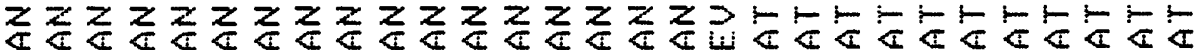

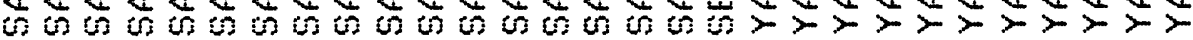

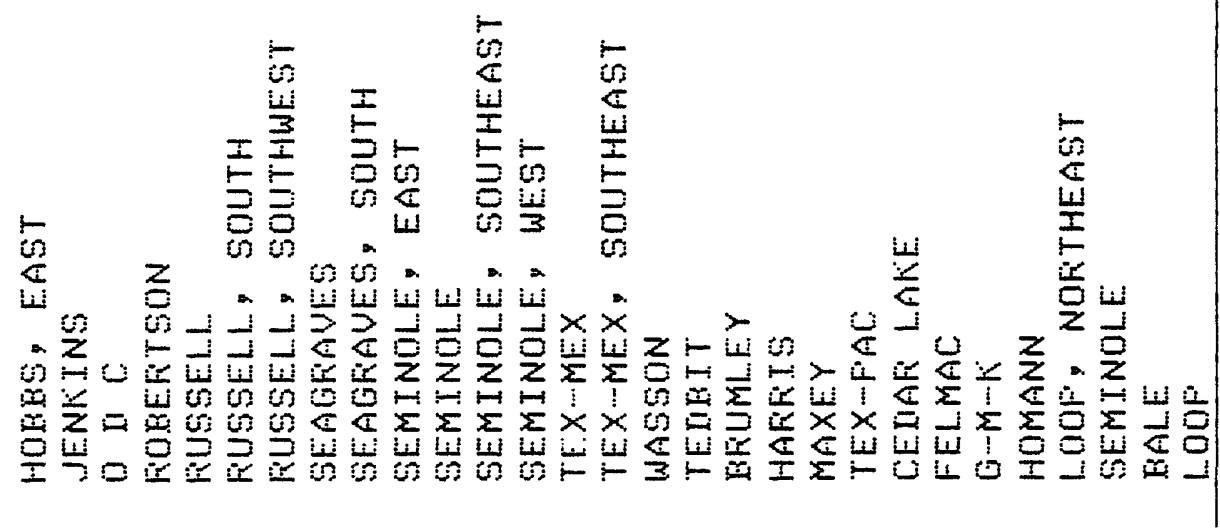

सरीNR रूल Nल⿵人丶

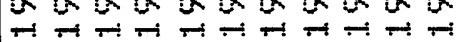

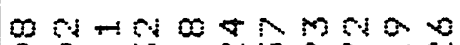

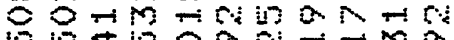

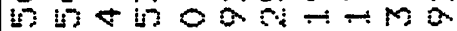

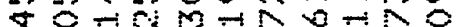

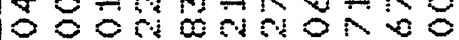

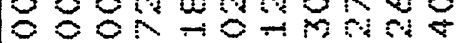
$6000 \div 8 \div \div \div \%$

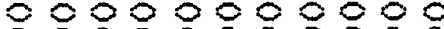

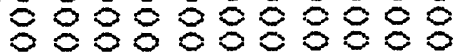
$00 \quad 002$

i5 800

8

riogison is 4 is 0 Thom 000 in $40 m$

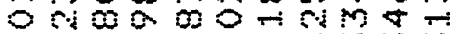

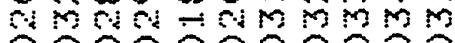
00000000000 - $0000-00$ : 000 ii) is 5 is is 000004 aरa 20000000

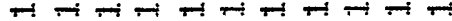

喵

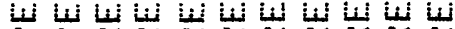

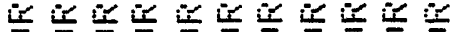

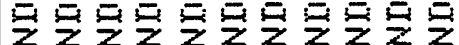

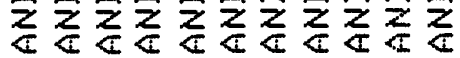

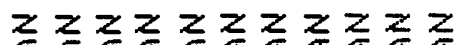

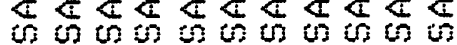

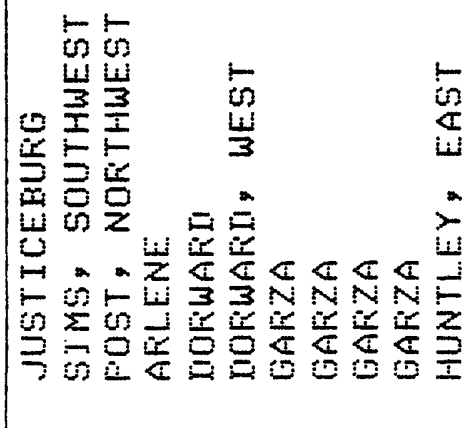




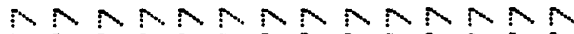
Nर⿵人र⿵冂人 2020202020200

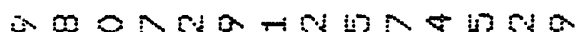

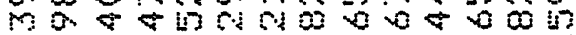
कल सकी

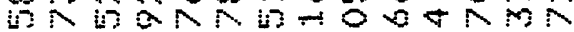

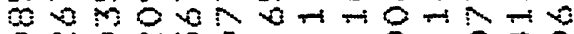
$0-6016 \div-96 \div 6 \div 0$

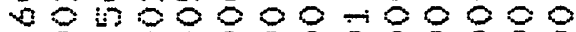

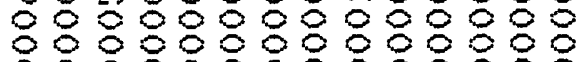
60060600600680 nobon on minis o

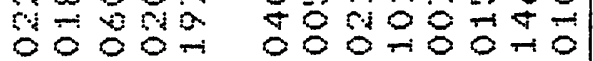
人

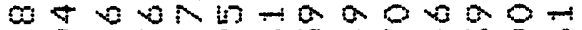

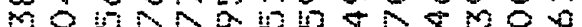
mo 0000000000000

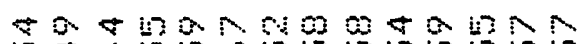

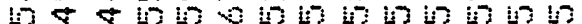

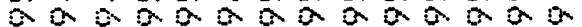

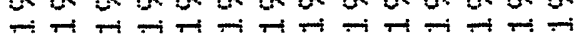

$\because \div$ $-1-3=$

$\Rightarrow \rightarrow=0$

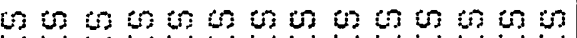
过

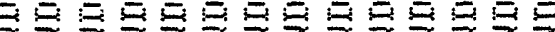

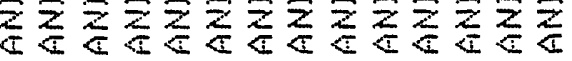

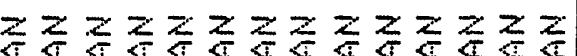
की

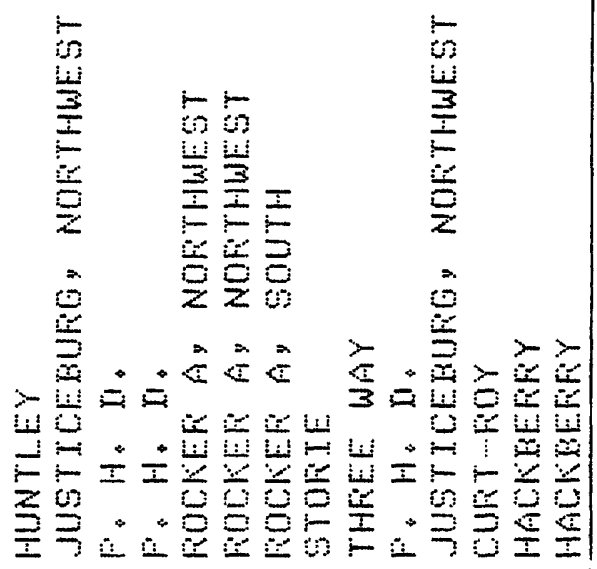

$\mathrm{NN} N \mathrm{~N} N \mathrm{~N} \mathrm{~N} \mathrm{~N}$

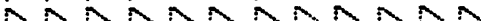
202000000000 THन

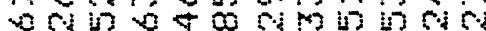

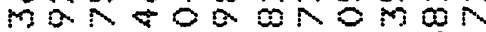
$0 m-R 00 M \wedge 000$

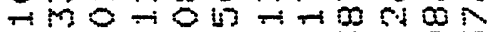
$006 \div$ : 68080080.080

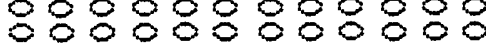

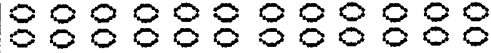

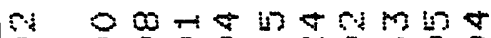
8 8. mom inow 0 . $0000 \Omega 0 \&+m N F$

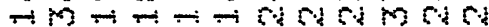
000000000000 4 of in is 00 is 0 00 000 0
0
0
0
0
0
0
0
0
0
0
0
0
0
0
0
0
0
0

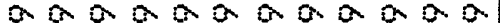

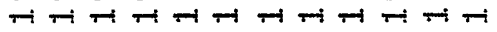

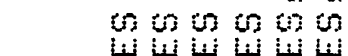

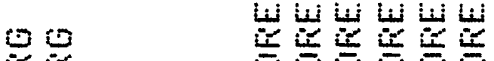
恶易

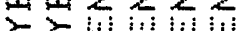

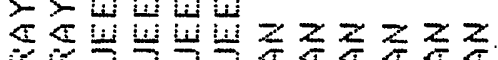

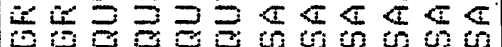

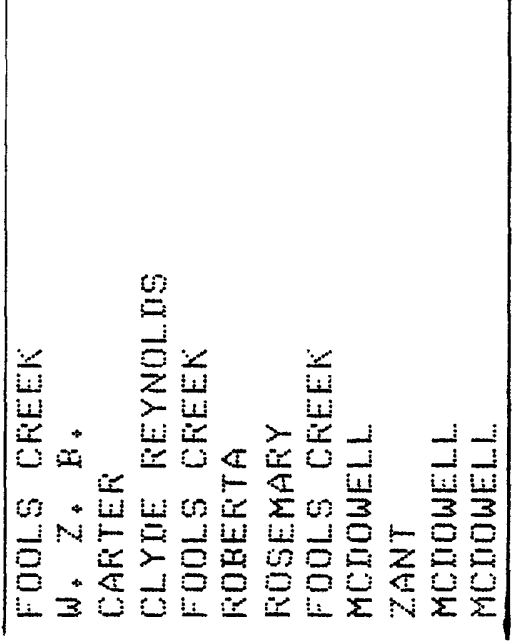

Wh WN लीo 200 $\rightarrow-i \quad+i$ $0+2$ माक $-6 m 000$ का in 500 \%० 080 क०० 808080 68ᄋ

mowomit i) $\rightarrow \div 00 \div$. $000 \div 000$ Mनi2 27120000 जम

mon $\mathrm{N}$ त i) a o 0 a in 20 $\rightarrow T \rightarrow+i-T \rightarrow$

की को का की

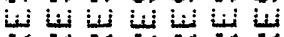

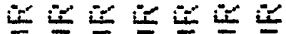

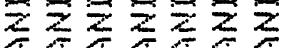

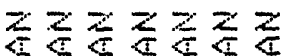
की $\mathrm{b}$ की $\mathrm{b}$

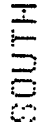

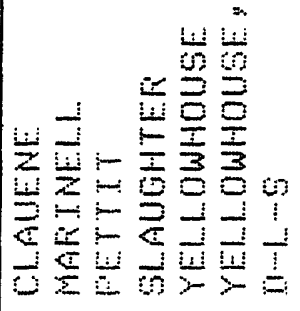




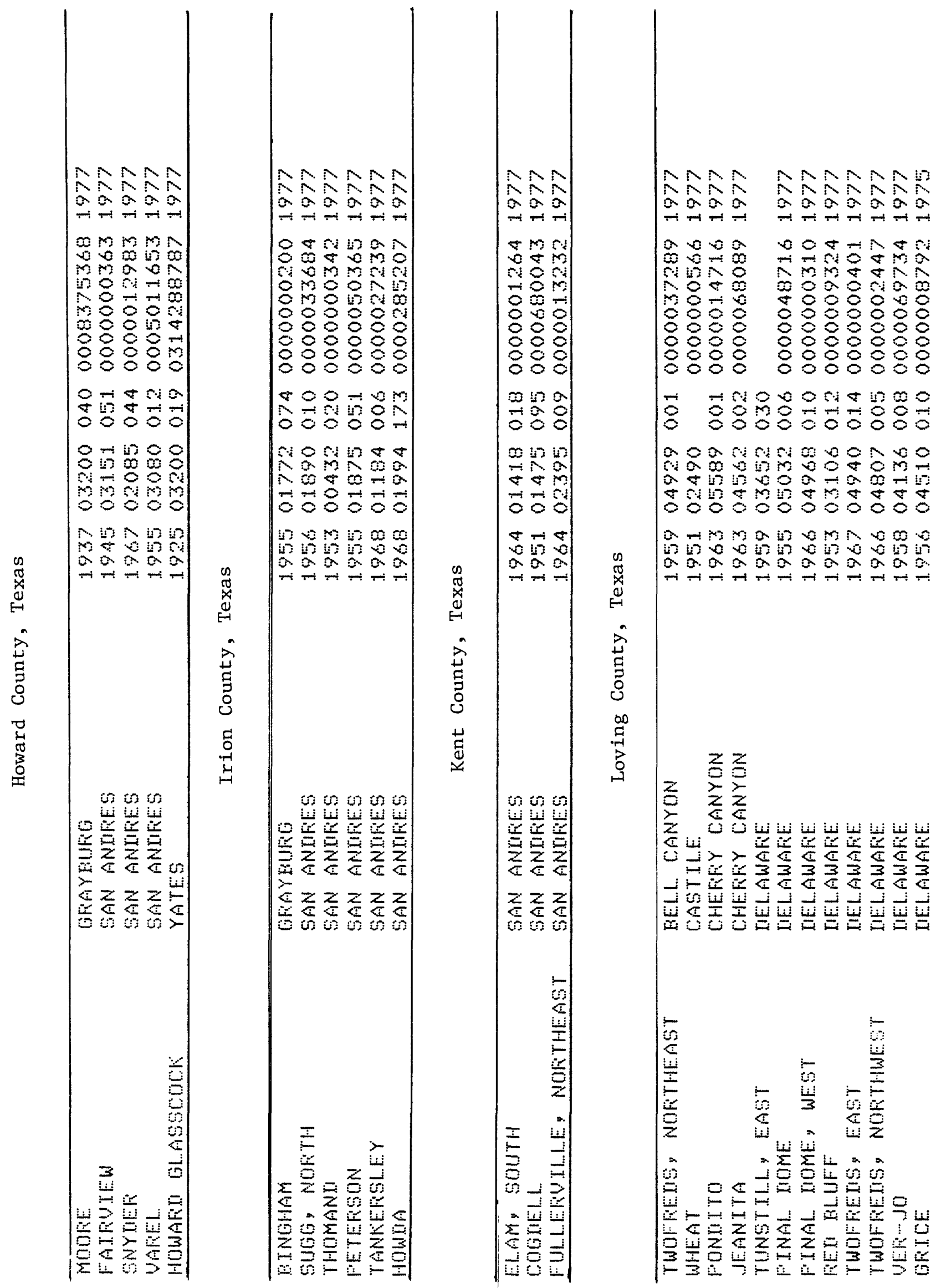




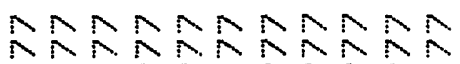

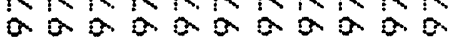

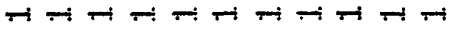
a $0 m \&$ in

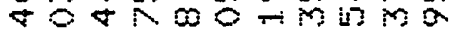
ना० ri $\rightarrow$ क तो 00 mat

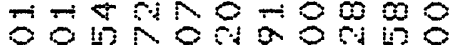
영

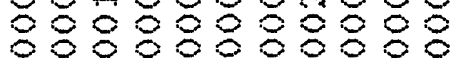

tmolnavonom

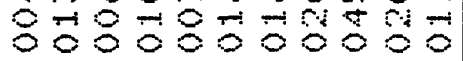

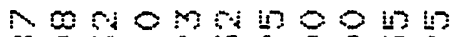
mom $\rightarrow$ an $000 \mathrm{~m}$ -in in 200 mo 00

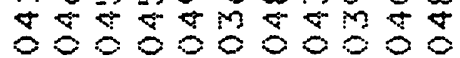
mab-a Nin 0 in 4 in 0 in कर 20202020

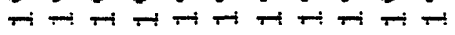

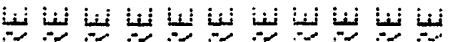

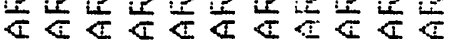

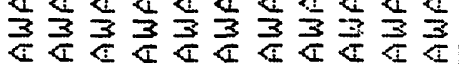

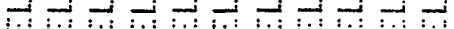

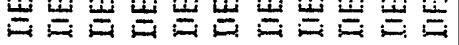

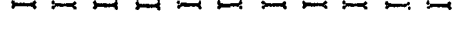

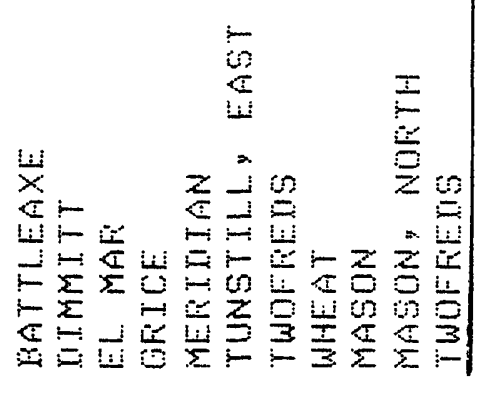

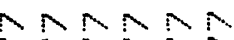

NNSNR

a 2020

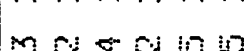

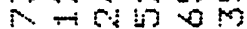

in $-10+\pi$

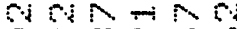

600000

8 in 080

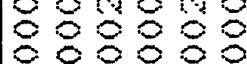

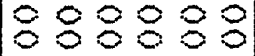

m: 0

$0-m=8$

* $280 \mathrm{~g}$

\&

806006

$\rightarrow+12000$

00 his

a 2000

年

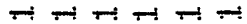



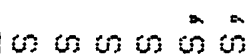

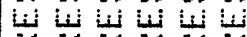

重语证

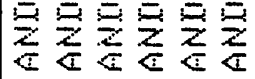

z진

60

(4) to 060

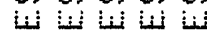

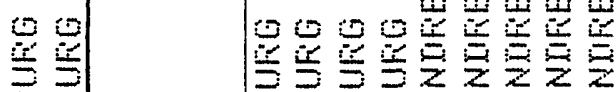

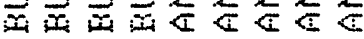

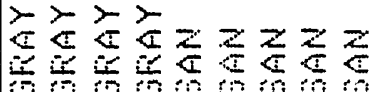
000000$$
\text { (1) }
$$

$\mid \begin{aligned} & 0 \\ & \vdots \\ & \vdots \\ & \vdots \\ & \vdots \\ & \vdots \\ & 0 \\ & \vdots \\ & \vdots \\ & \vdots \\ & \vdots \\ & \vdots \\ & \vdots \\ & \vdots \\ & \vdots \\ & \vdots \\ & \vdots\end{aligned}$
WNAR NR कर $202 \alpha 200$

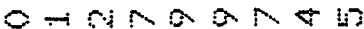
\& $8030800 \%$ 00060001

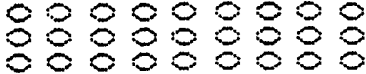
00000000 स्व. \%क 6000-10n-in

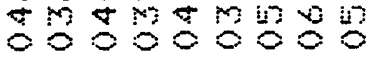

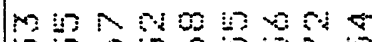
in is 0 is 0 in 170 iो 200000000 Ti

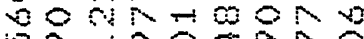

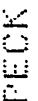

$\geqslant \quad 05 \quad 4 \pi$ 는 $\div 4 \leq 4$ $2 x \sum \sum \geq$

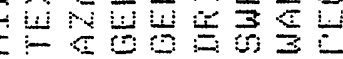




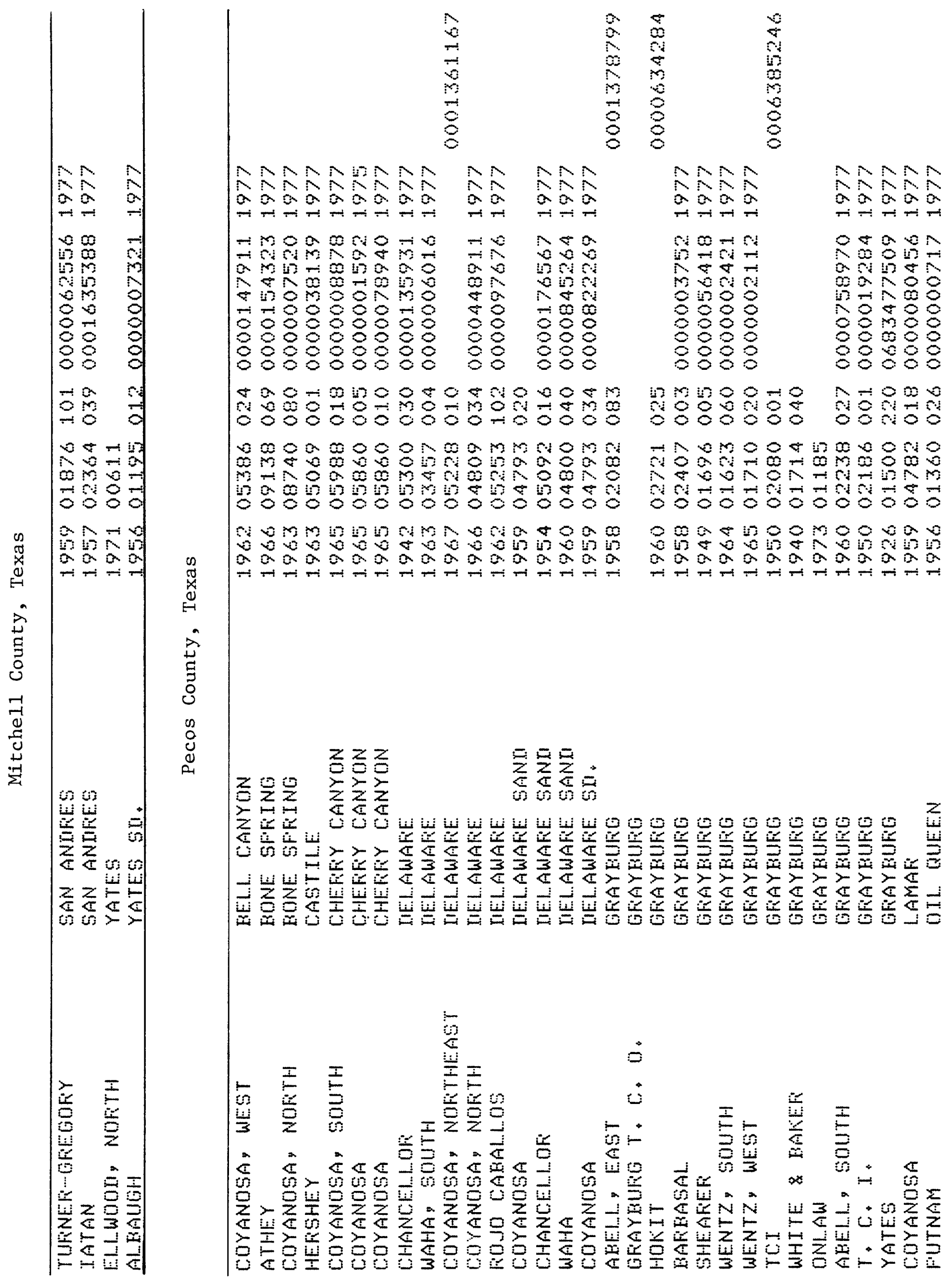




\begin{tabular}{|c|c|}
\hline $\begin{array}{l}4 \\
3 \\
5\end{array}$ & 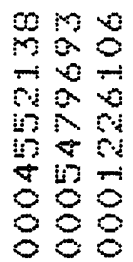 \\
\hline
\end{tabular}

NRN N

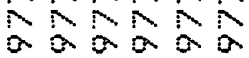

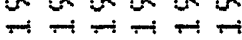

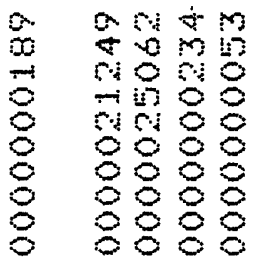

a $0 m \infty \mathrm{m} ; 2 \pi$ in 100848884
NNSWNSNRNR a 200200020200

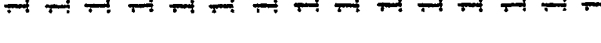

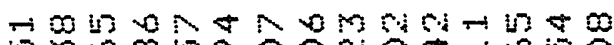
in $201200000 \%$ क ma cac \& m \&

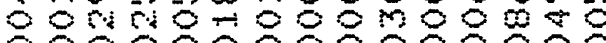

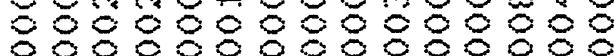

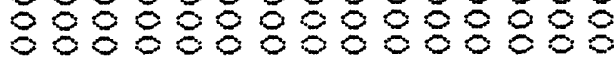

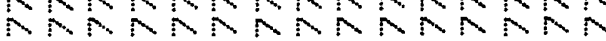

$\mathrm{NSN}$ $\alpha a \alpha 2$ $\rightarrow+1 \rightarrow$

$0 m \& i 5$ $8 m i n$ $01 \infty$ $8+00$ 8688 8888 $\begin{array}{ll}\infty & 0 \\ 0 & 0 \\ 0 & 0 \\ \infty & 8 \\ 0 & 0 \\ 0 & 0\end{array}$

$\mathrm{NMN}$ 2. 20 $\rightarrow+i$

$N m a r$ 2050 $2 \div 00$ in 2013 त 0500 8880 6808 in की

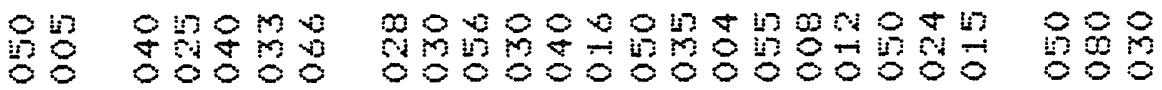

Nomon man

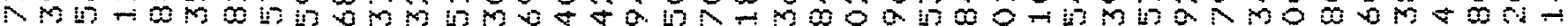

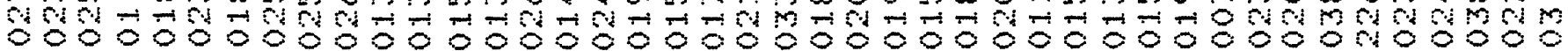

Non-ism-

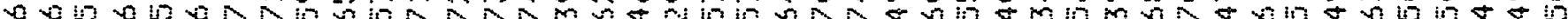

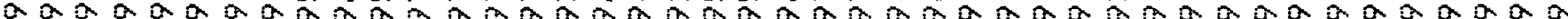

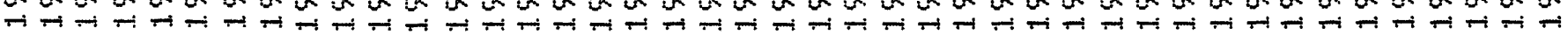

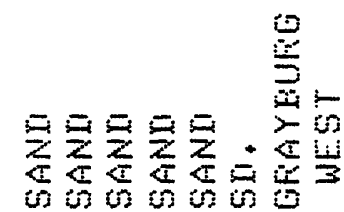

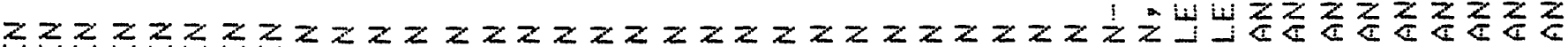

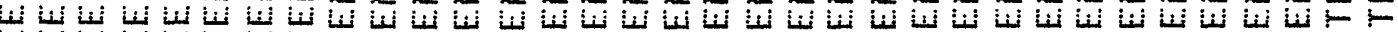

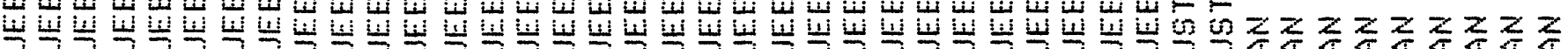

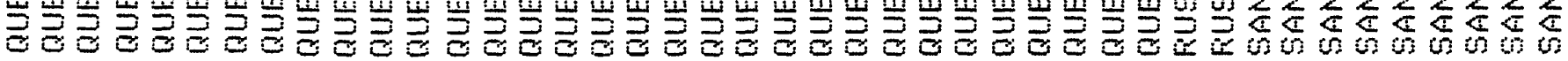

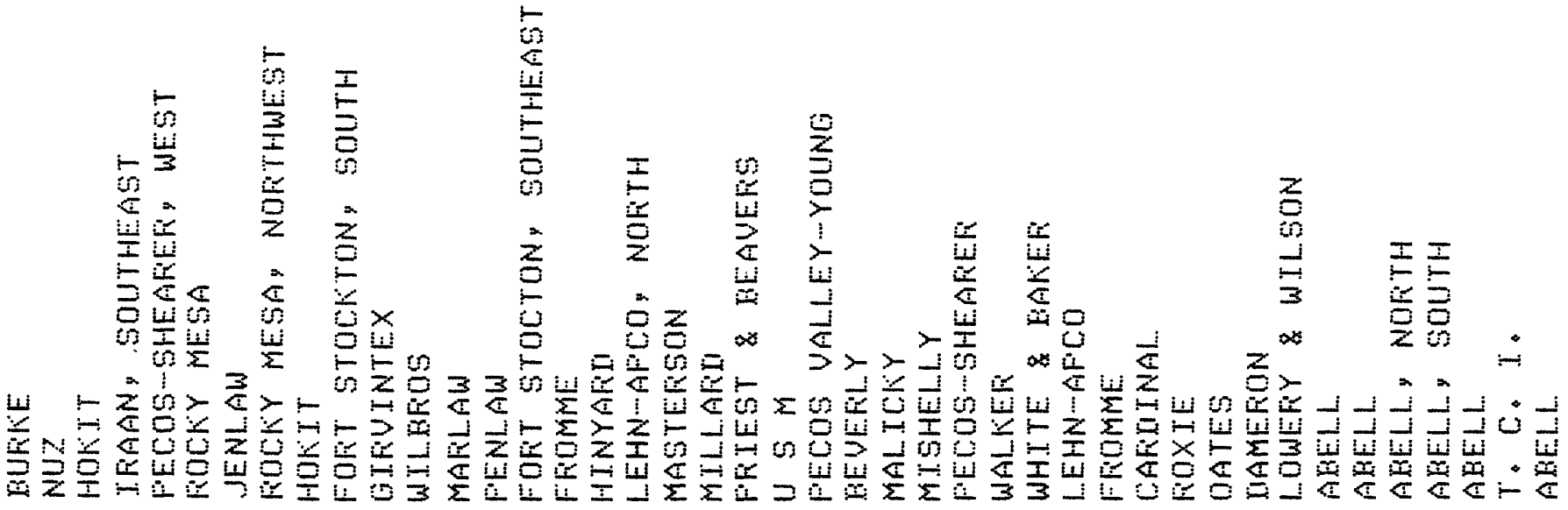




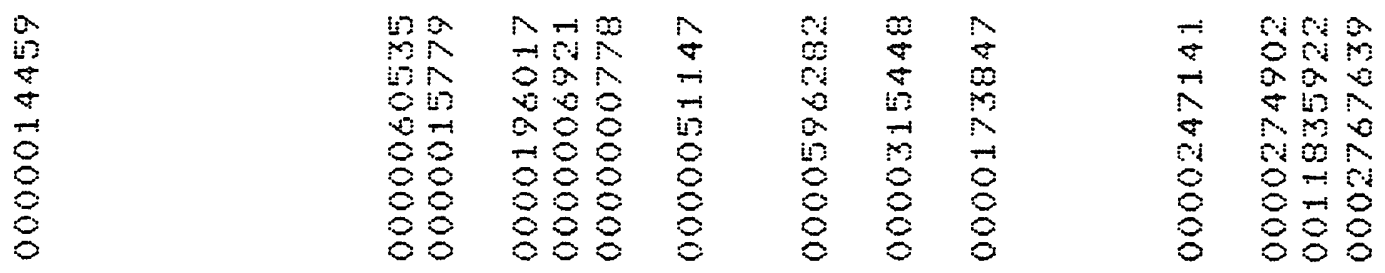

NA RNAR

a 20020

$\hat{2} \quad \hat{2}$

NAN

2022

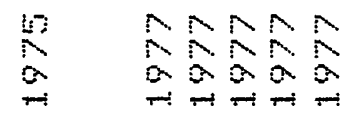

$\rightarrow \rightarrow \quad \rightarrow \rightarrow \rightarrow \rightarrow$

$\frac{8}{8}$

Na $20 \%$

-1

i1 0 क 00

88 द 80

88 पेकेठ

88

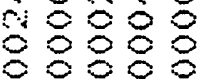

0
0
0
0
6
6

$00 \%$

$8 \infty$

का m

ㄷㅇㅇ영

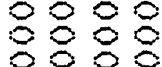

8888

mmष

i.

काल

$0 m \infty m$

$\infty \sin \rightarrow \infty 0$

तथा

$800 \%$

88080

86888

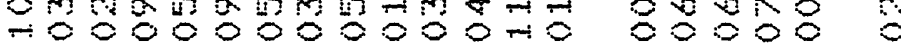

(3)

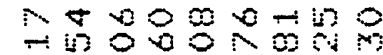

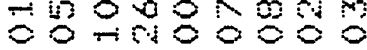

Hooomoooom

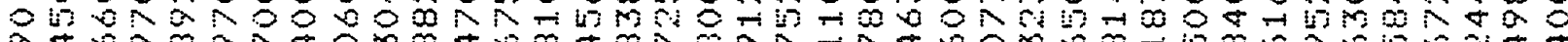

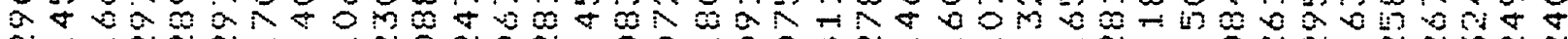

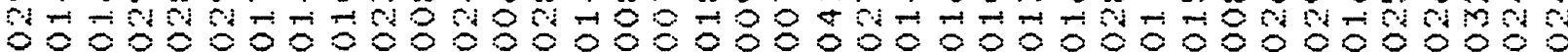
00 -

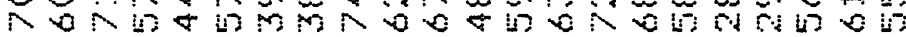

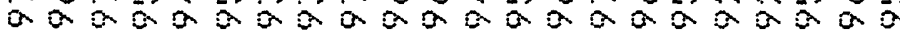
$\infty 0 \div 0$ is

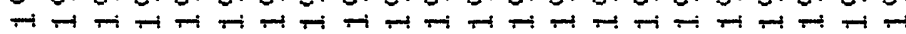

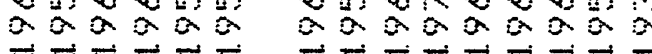

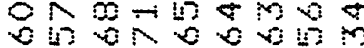

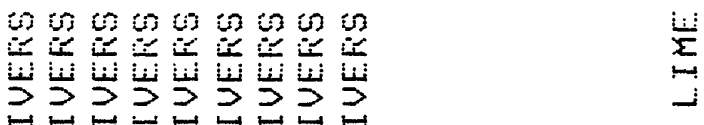

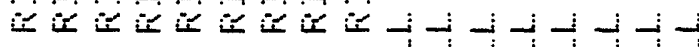

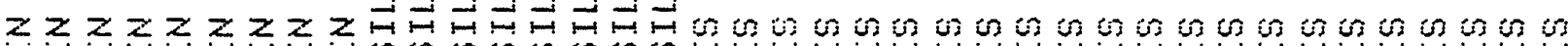

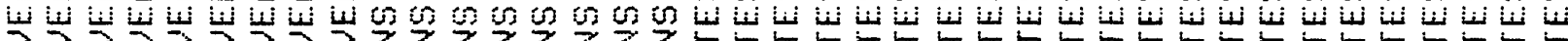

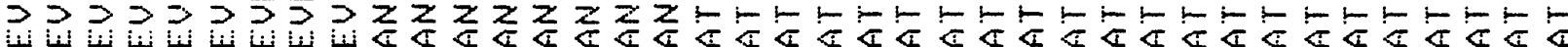

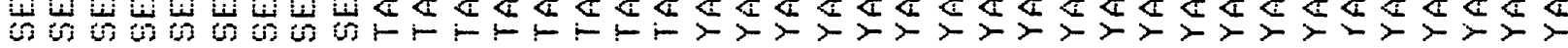

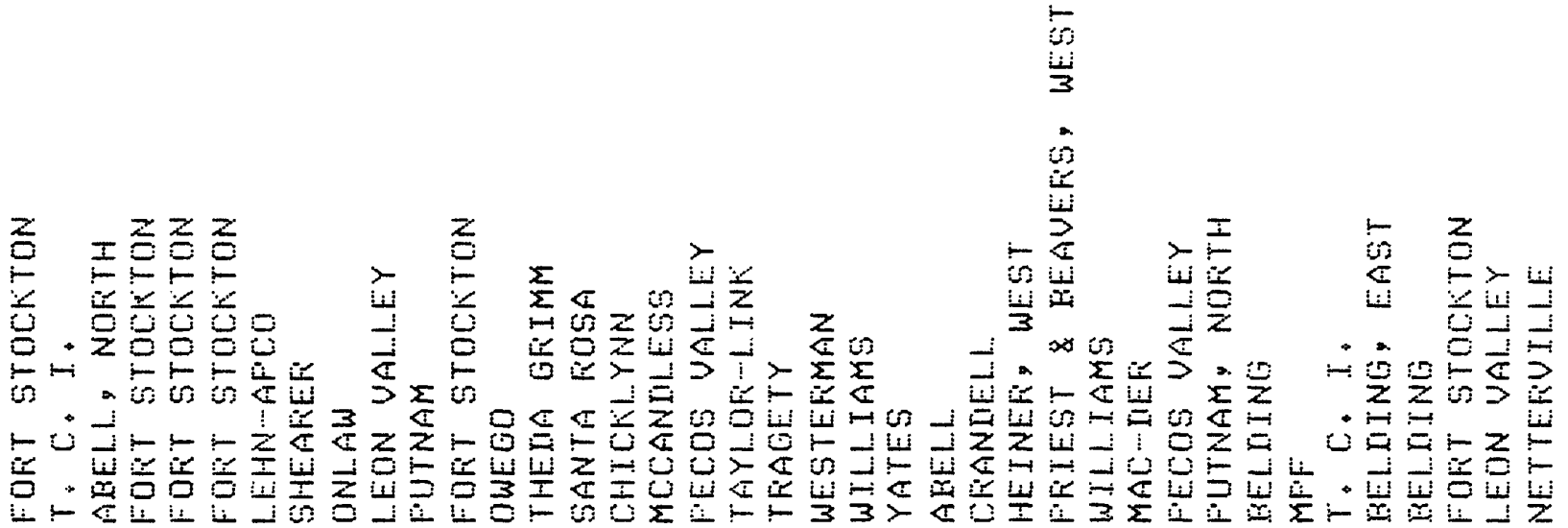


NरीNR

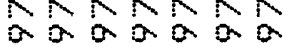

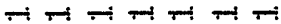

以

$m \infty 1000$

O\& 0 री

$+\pi \infty \infty=0$

- 0 in

6.

$0 \% 0080$

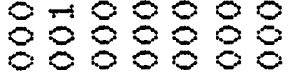

i) 3000060120

i) 0 मn mori mo6riman i $\infty 0 \pi 0 m \infty 0$ -

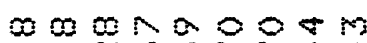

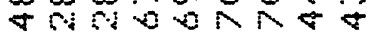
a. 20002002 $\rightarrow \rightarrow \rightarrow \rightarrow \rightarrow+\rightarrow \rightarrow+4$

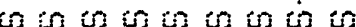

0
0
0
0



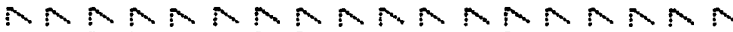

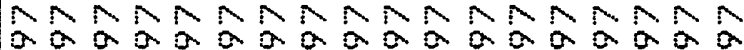
다 بा

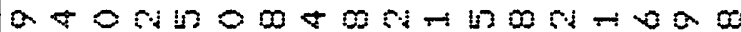

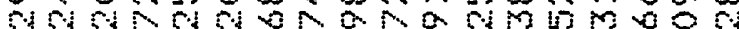
की

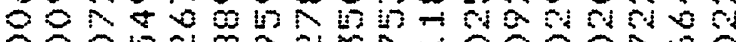

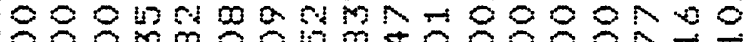

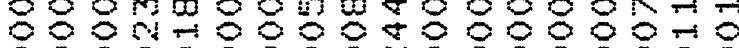
$989899698 \% 8989896$

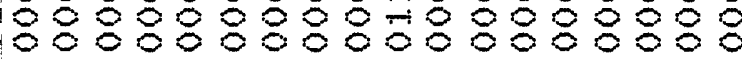
mámorषष

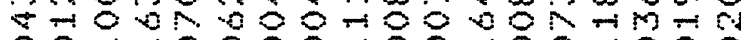
m. -0 i

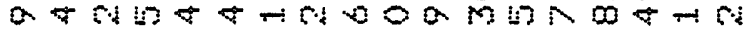
जr o00000000000000000

in + mm+ 4.

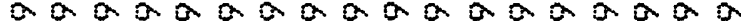

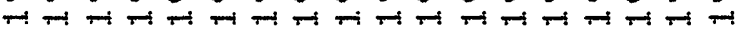

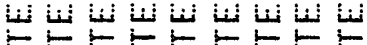

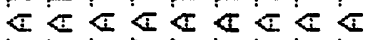
$x \dot{x} x<x$

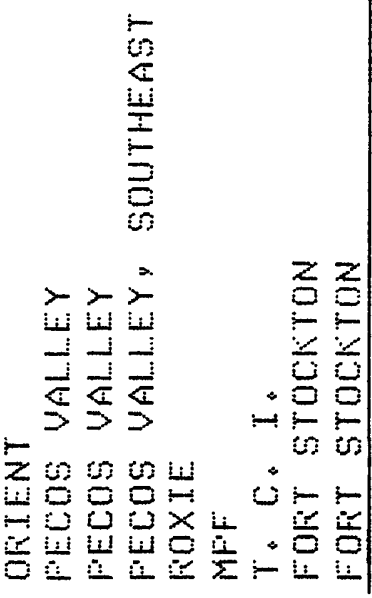

6 to th th th th th

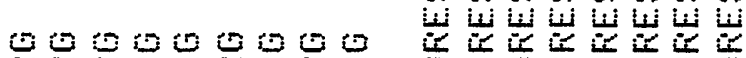

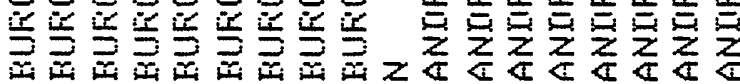

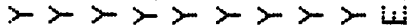

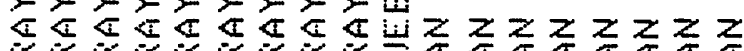

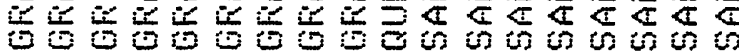

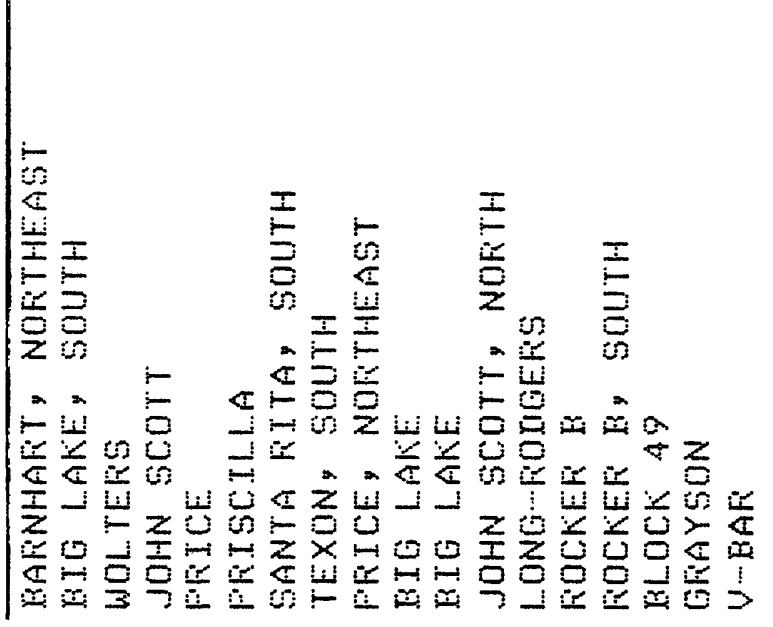


00
80
80
80
8
8
8

NNN WBNNAWHWNANR ANA DNANANRRANAN 2020020202020200 $\infty \overrightarrow{0} \square$ Sक 106 68 880 $8 \% 8$ 888 $\begin{array}{ll}4 & 8 \\ 0 & 8 \\ 0 & 8 \\ 7 & 8 \\ 8 & 8 \\ 8 & 8\end{array}$

in 20

$\infty$

$\stackrel{2}{\infty}$

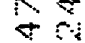

68

88

88 88
NANNANNMN WNAEANANA 20002020000

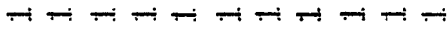
$\sin \mathrm{min}+\mathrm{an}$

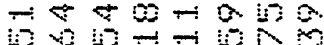

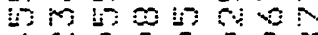
- m00000\%

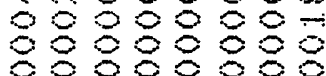

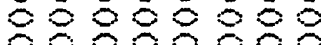

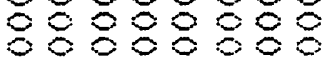$$
\begin{array}{lll}
7 & 0 & \\
6 & 0 & 8 \\
8 & 0 & 8 \\
8 & 8 & 8 \\
8 & 8 & 8 \\
8 & 8 & 8
\end{array}
$$

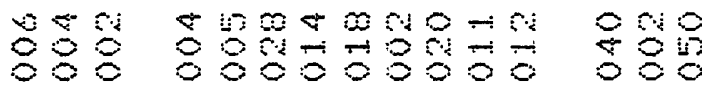

6

6 त0 $\begin{array}{ll}0 & 0 \\ 8 & 0 \\ 0 & 0 \\ 0 & 0 \\ 0 & 0 \\ 0 & 0\end{array}$

in.

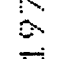

i2

का m-56,

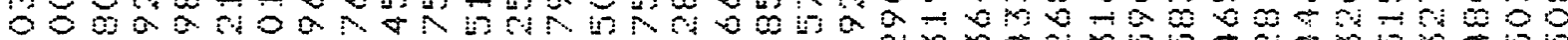

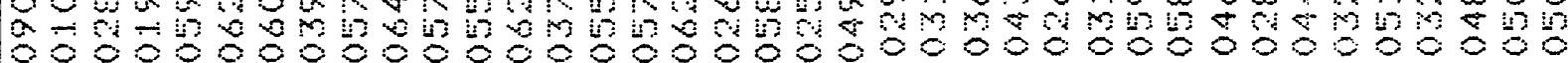
ma $0+\infty$ -

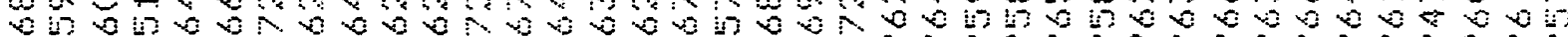

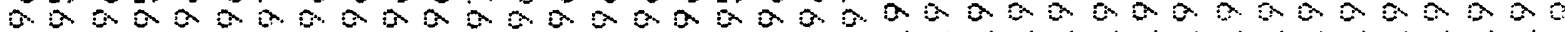

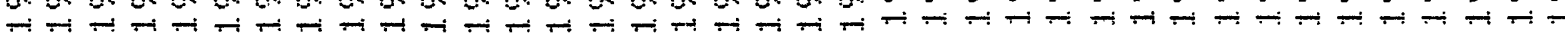

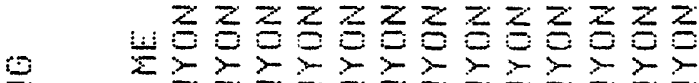

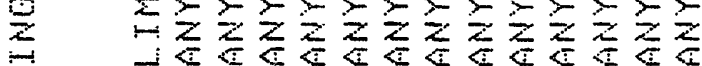

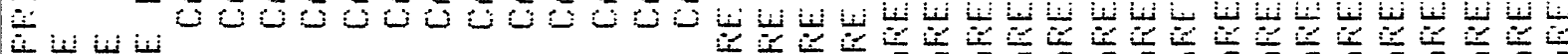

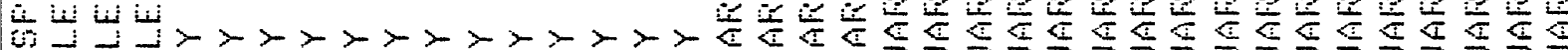

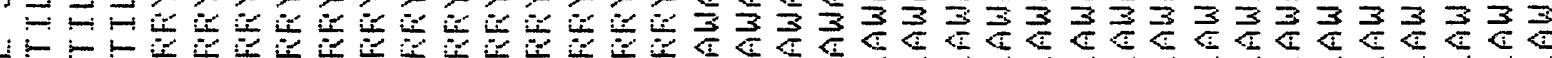

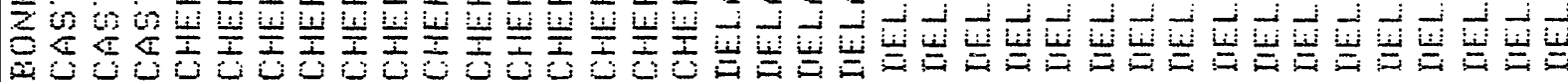

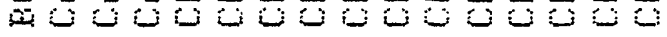
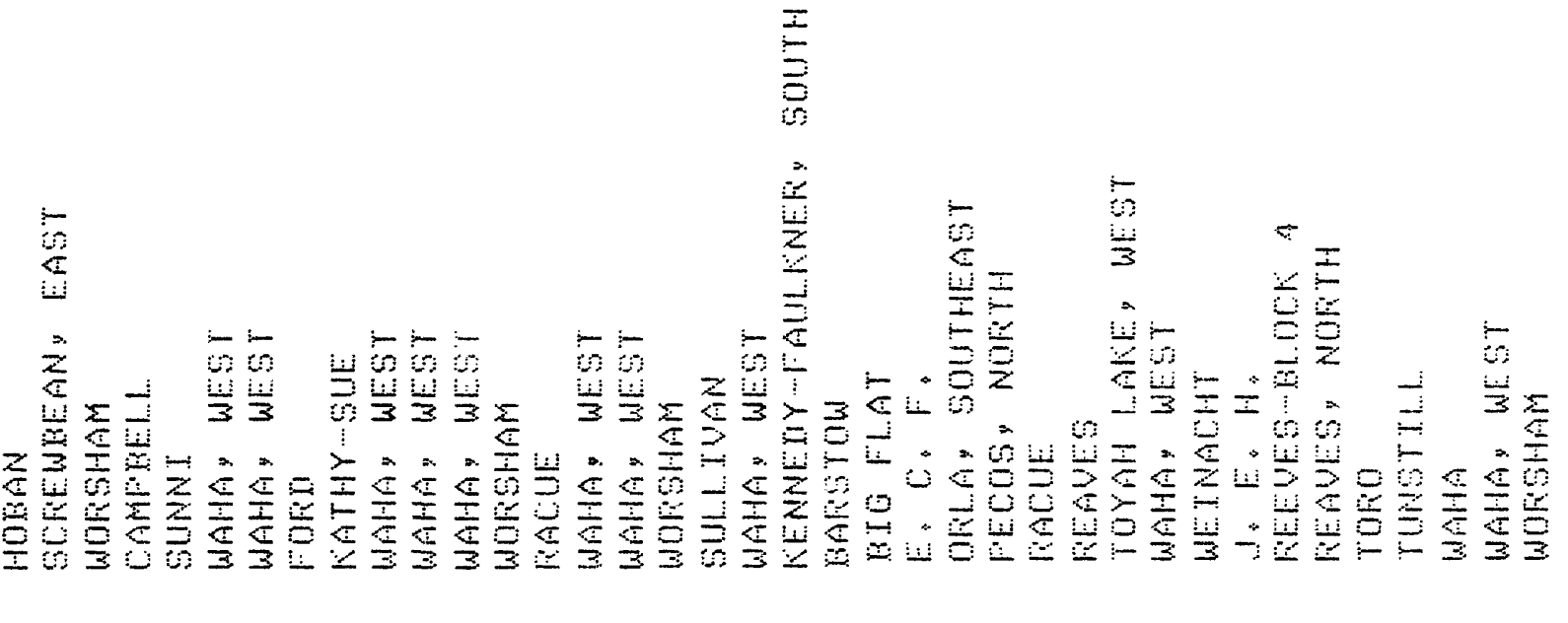
$\begin{array}{ll}10 & 0 \\ -1 & 0 \\ 0 & 0 \\ 0 & 8 \\ 8 & 8 \\ 8 & 8 \\ 8 & 8\end{array}$

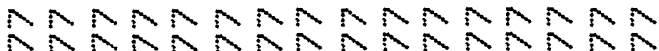
$\alpha 0 \alpha 0000000000000$

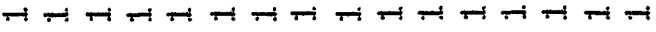

Thin 0000 क

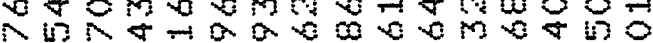

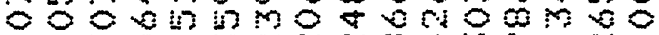
राm

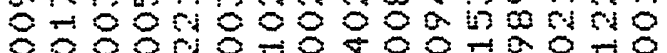

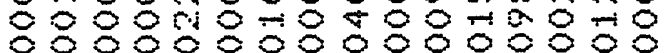

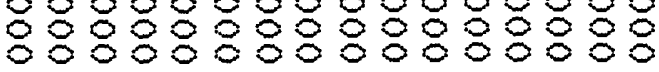

THFT

$\cos a$ 10000 0000 N $\rightarrow 0 \div$ 0000 8000 8080

NSRSRSRN $\alpha \alpha \alpha \alpha \beta \alpha \alpha \beta \alpha 0$ तन

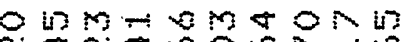
का दर

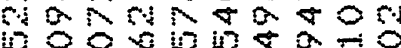
0 जा का

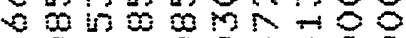

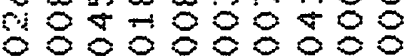

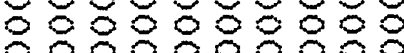
m

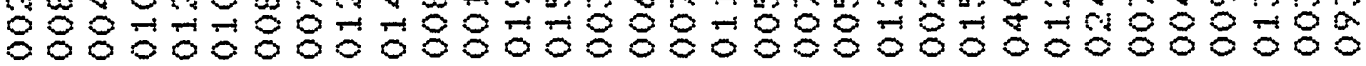

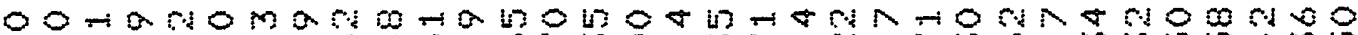
60.

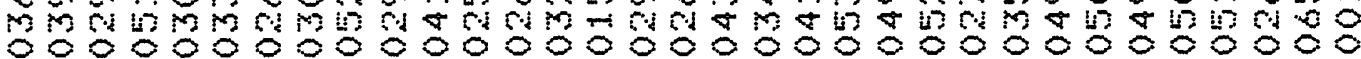

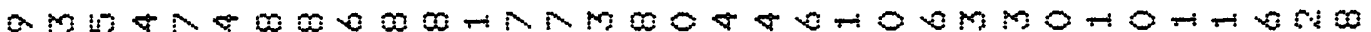

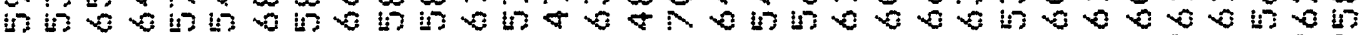
0000000000000000000000000000000000

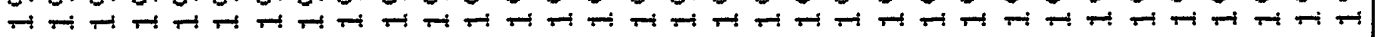

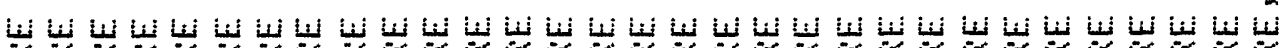

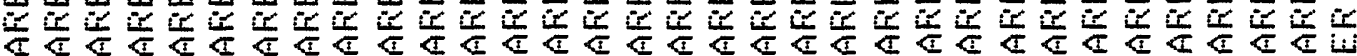

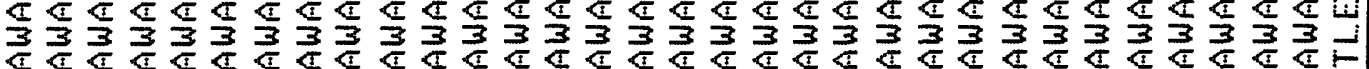

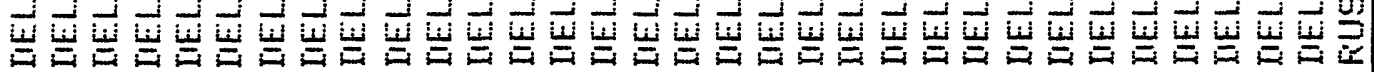

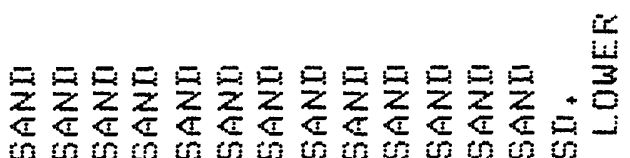



रNล2 2000200

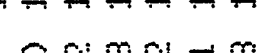
820020 तi is $0 \rightarrow \infty \infty$ $80 m \infty-1 ;$ $800 \mathrm{~m}-\mathrm{s}$ $0+1-00$

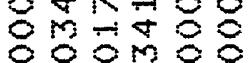
800080 i) 0 ती

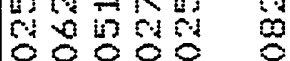
$06200 \pi \infty$ Nom 0 in is $\rightarrow 0$ MN

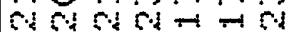
0000000

onmoms $\rightarrow$

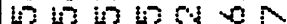

on

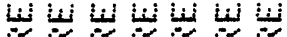
证送这

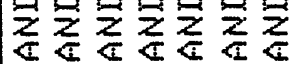

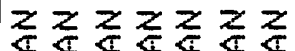
4605640
NNNN

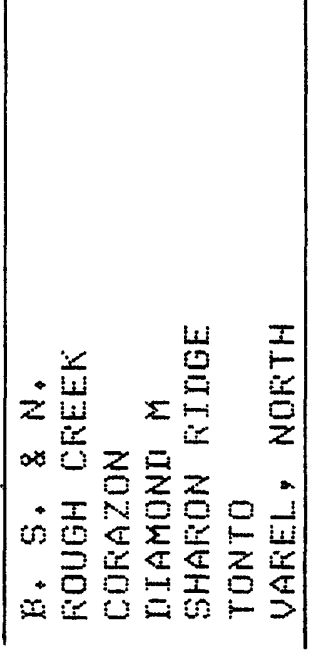

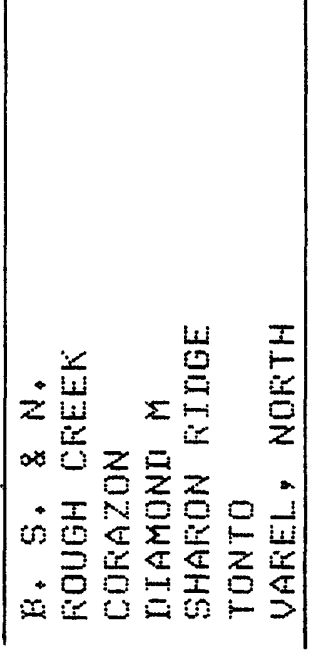

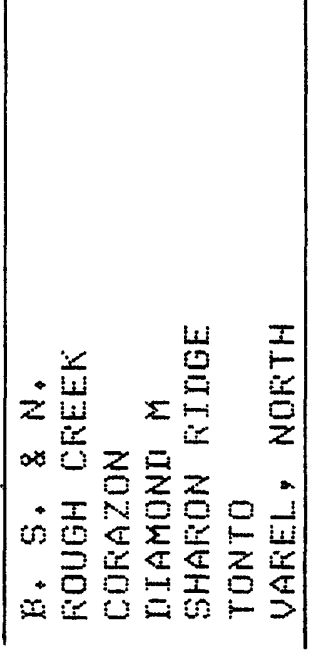

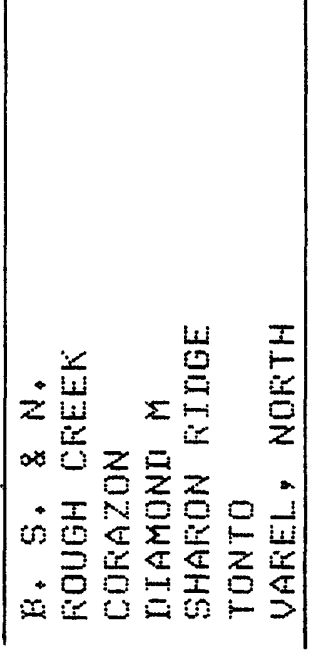

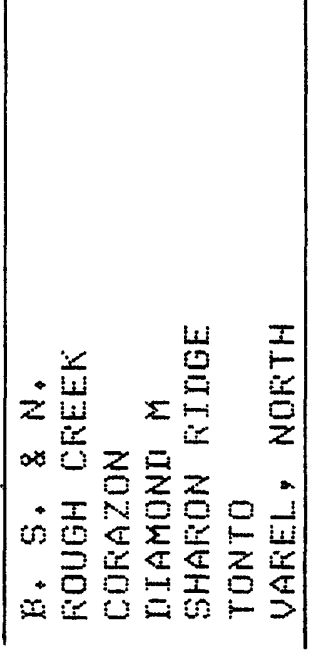

तNDNANANAN

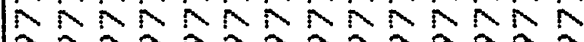
2020202020020 an-amanN+4mono

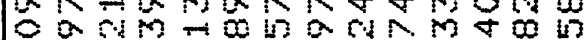
तीm ४ manomomrio\& on Un 0808

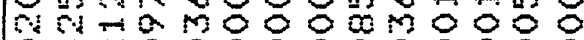

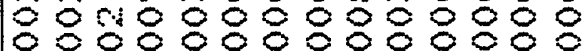

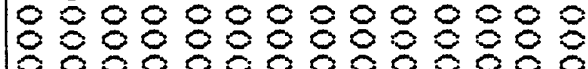

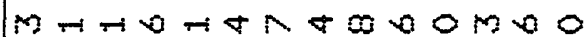

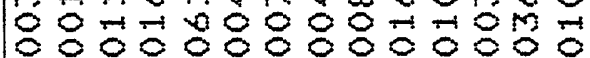
-

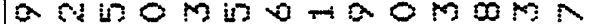
$0 m \&-i m \& \& M \infty \& N a+\infty$

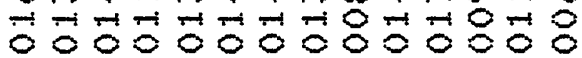
एin $\rightarrow+m a n a 0+$ in

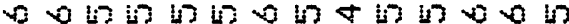


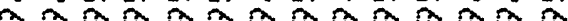

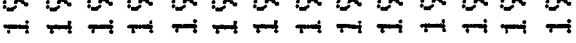

\section{$\Xi$}

$\equiv \equiv 0$ क

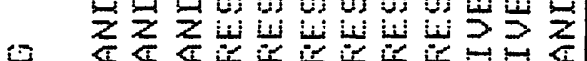

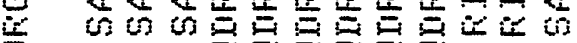

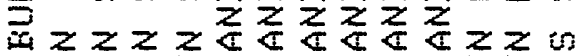

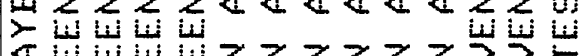

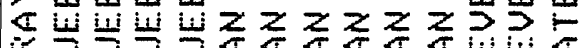

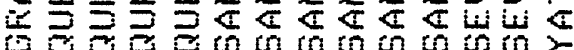

$\frac{5}{3}$

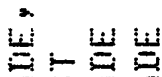

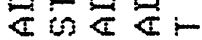

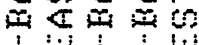

$\frac{1}{\pi}+\frac{11}{3}$

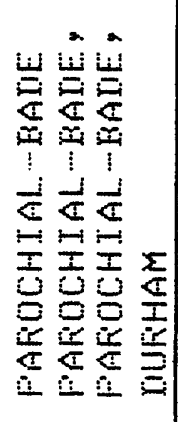

$\begin{array}{ll}8 & + \\ 2 & 1 \\ 8 & 0 \\ 8 & 0 \\ 8 & 0 \\ 8 & 0\end{array}$

$\mathrm{N} N$

$\sum_{0} N$

202

$+\infty \infty$

$\left\{\begin{array}{lll}0 & 0 & 0 \\ 0 & 0 & 0\end{array}\right.$ $\rightarrow 0$ 200 $\because \rightarrow \infty$ 888 800 is 0 कo $02 m \infty 00$ C०कलM Oij 600000

00000 1700000 a $\log 2 \pi$ $\rightarrow+\rightarrow+T \rightarrow$

60 in

ij

国

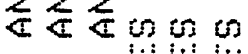

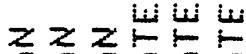
$\bar{\tau}<\varangle \varangle$

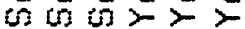

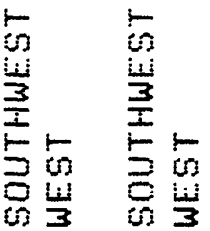
$z$

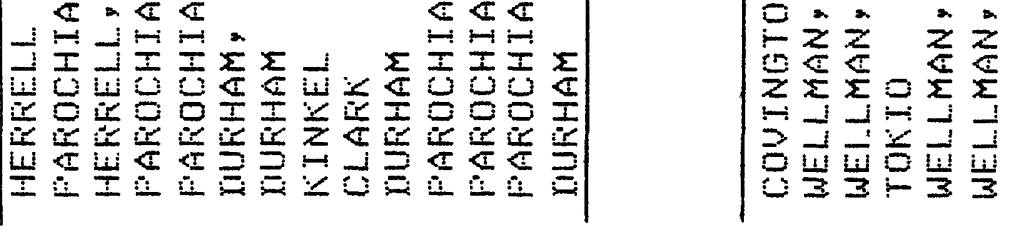




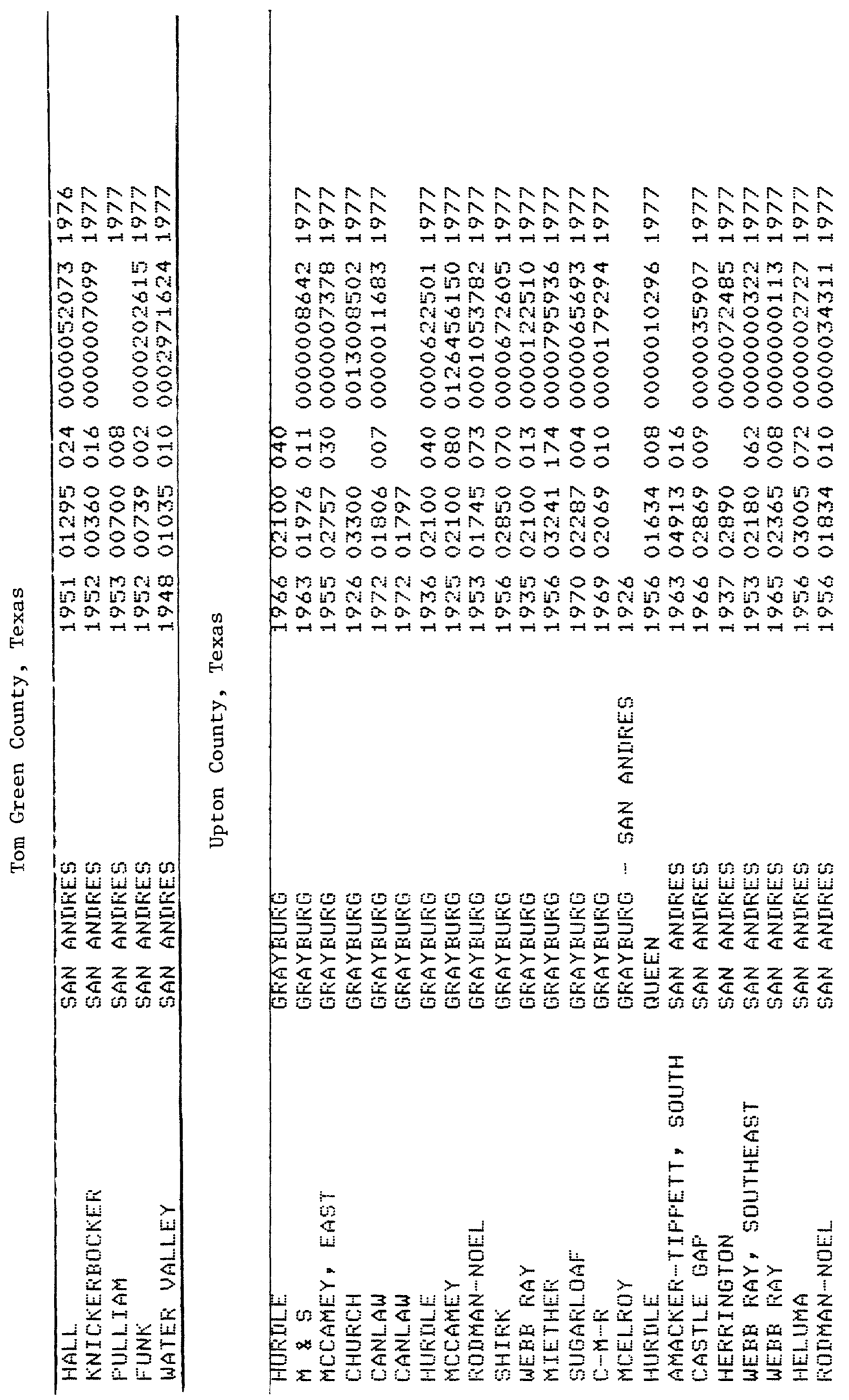




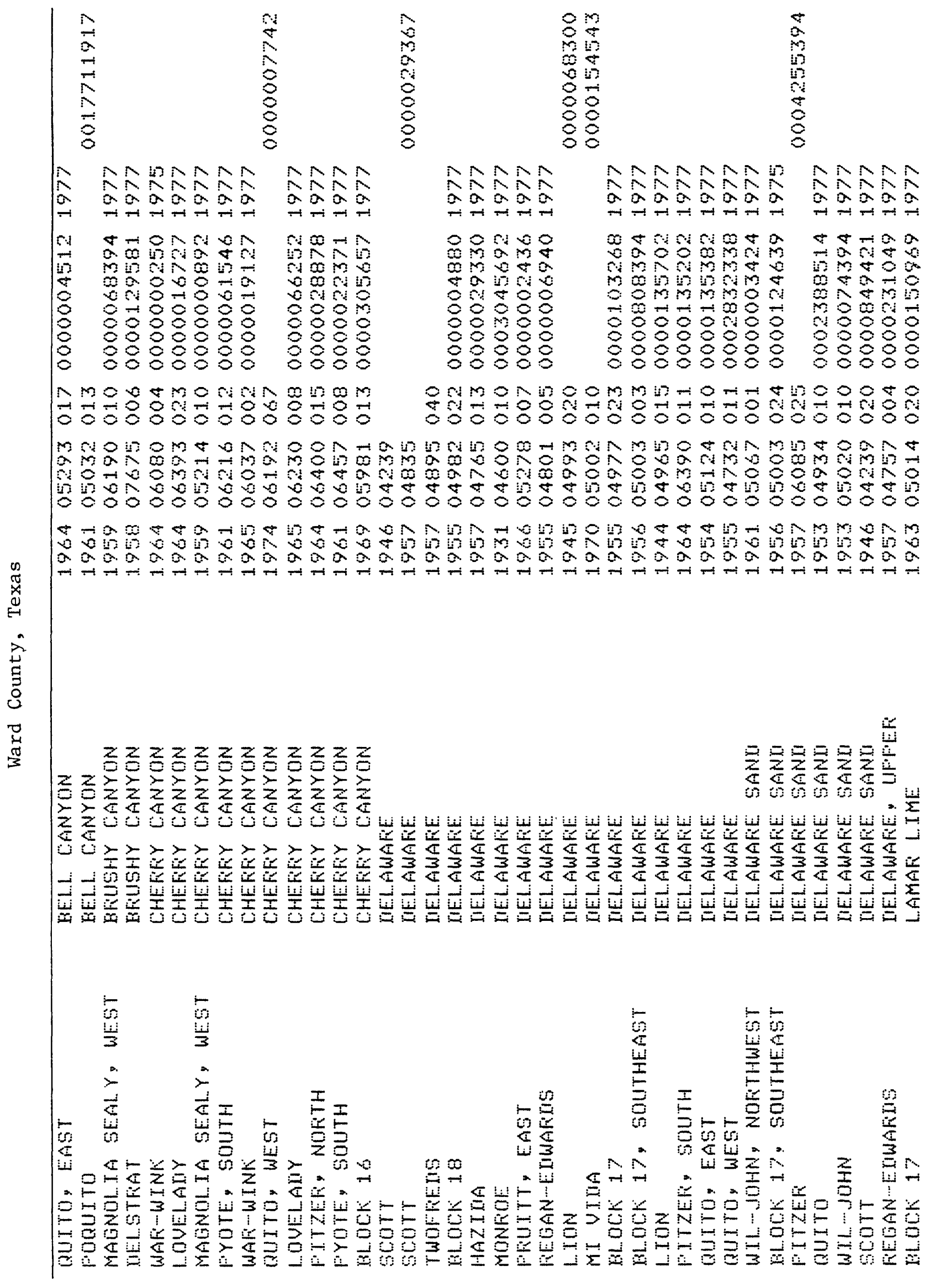


ANANANARANANA

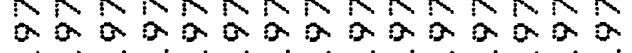

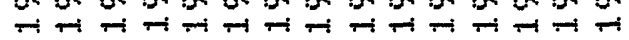

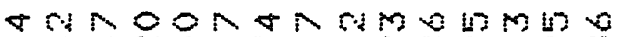
कo nom औक

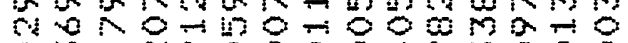

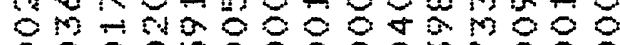

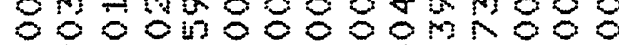

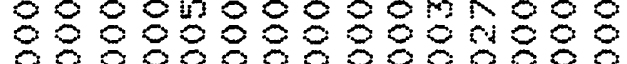

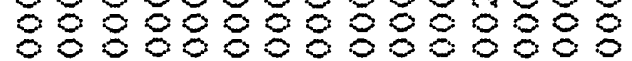

NA NNNE

NA NANAR

202022

$\infty m \infty \infty+120$

$\infty$

क्ष

$\infty$

mo

08

80

88
2
0
0
8
8
8

$N$

20 ती

$\rightarrow \pi \quad \pi-\pi$

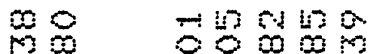

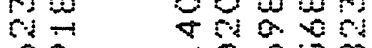

क 0 त0

- 8 misos

ab जin

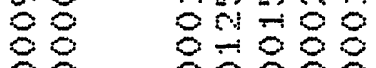

08080

샹ㅇㅇㅇ

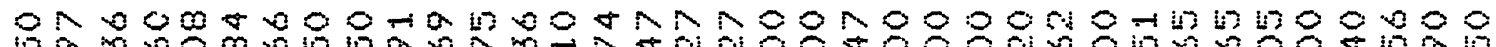

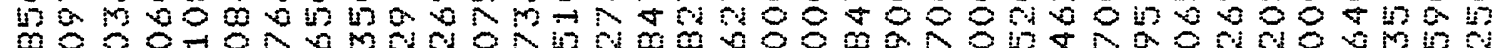
कo ग0

Non

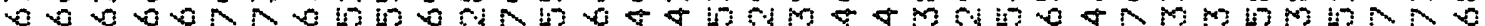

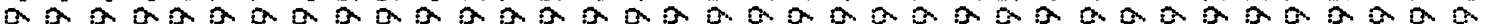

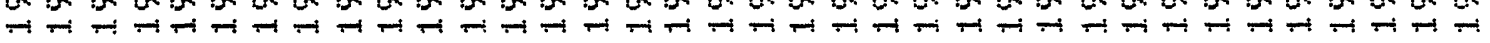
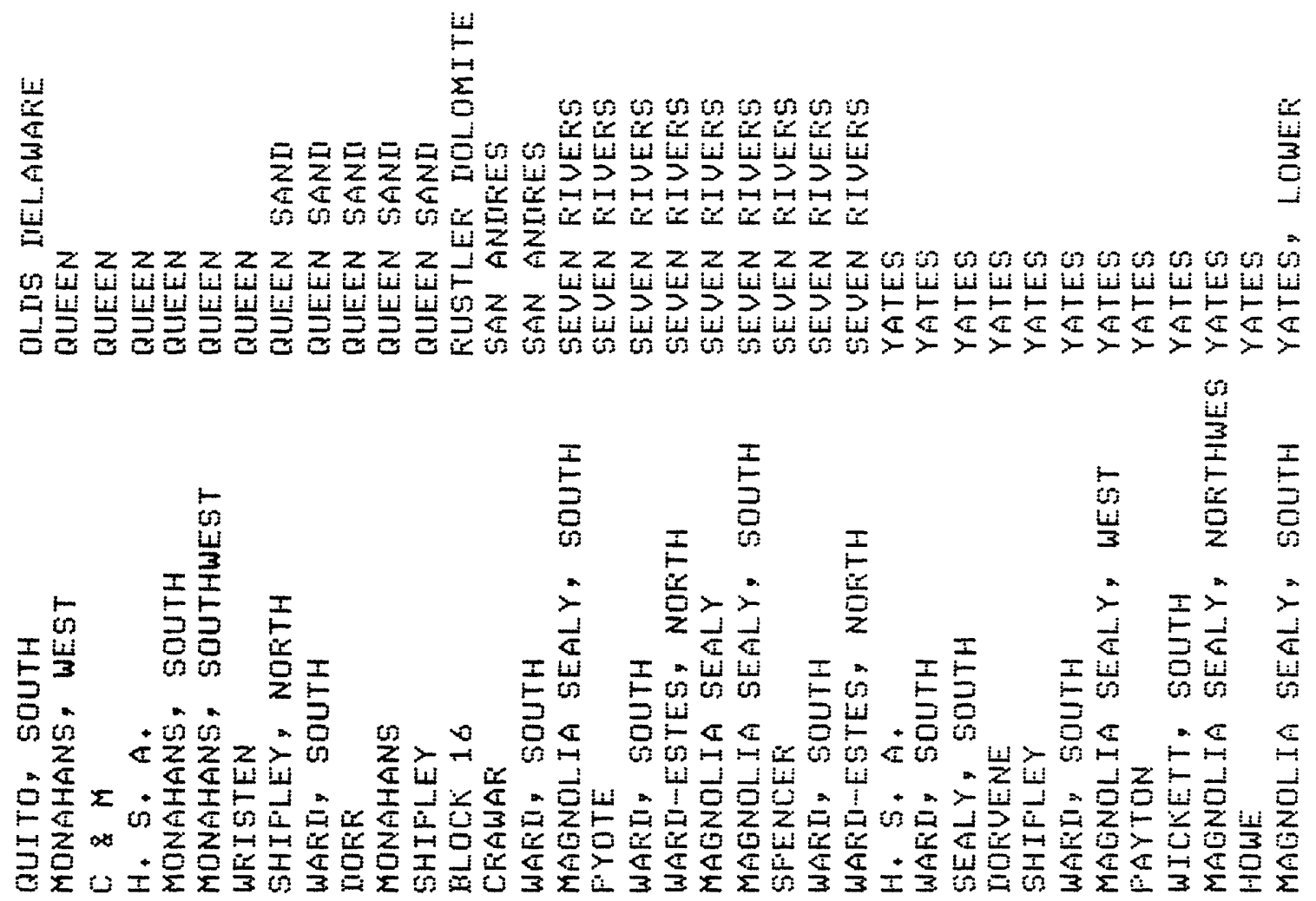


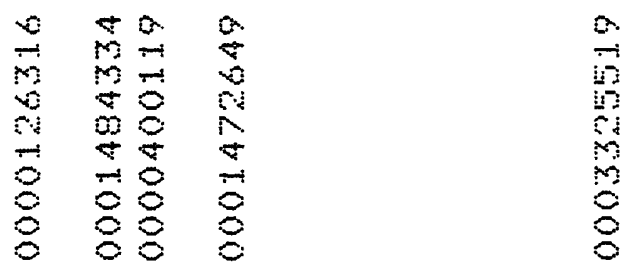

$\begin{array}{ll}0 & 0 \\ 7 & 0 \\ 17 & 8 \\ 0 & 8 \\ 8 & 8 \\ 8 & 8\end{array}$

\begin{tabular}{|c|c|c|c|c|c|c|c|}
\hline$\hat{2}$ & 2 & 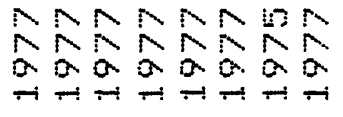 & $\stackrel{1}{2}$ & $\hat{2}$ & $\hat{2}$ & $\begin{array}{l}2 \hat{2} \\
2 \hat{2}\end{array}$ & 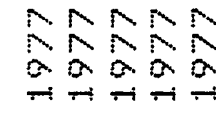 \\
\hline $\begin{array}{l}0 \\
0 \\
0 \\
6 \\
6 \\
0 \\
0 \\
0 \\
0\end{array}$ & $\begin{array}{l}2 \\
0 \\
8 \\
8 \\
8 \\
8 \\
8\end{array}$ & 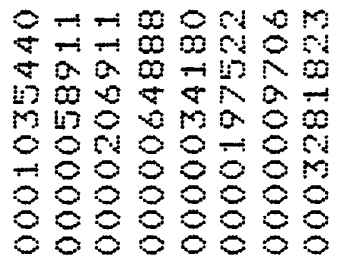 & $\begin{array}{l}8 \\
8 \\
8 \\
8 \\
8 \\
8 \\
8\end{array}$ & $\begin{array}{l}80 \\
08 \\
0.5 \\
8 \% \\
8 \\
8 \\
8 \\
8\end{array}$ & 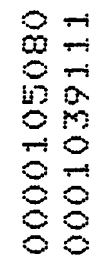 & 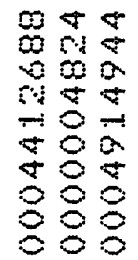 & 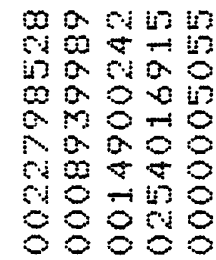 \\
\hline
\end{tabular}

समीय

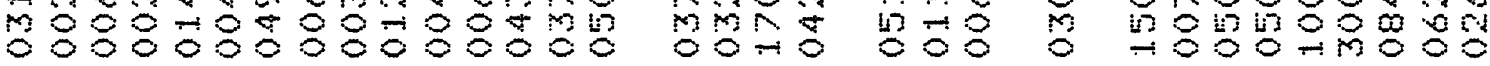

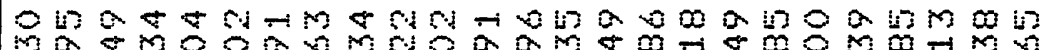
mo D. 0000000000000000000000000

Ma

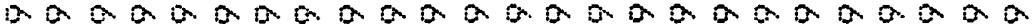

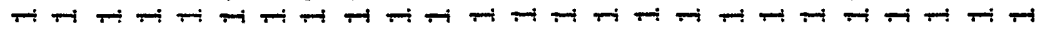

$88808880 \mathrm{~m}$

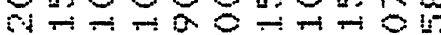
mMmmMmmis 00000000000

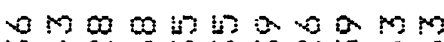

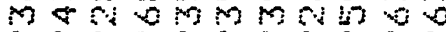
20202000000

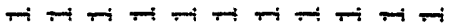
$\stackrel{\square}{\frac{1}{3}}$

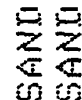

$\frac{9}{2}$

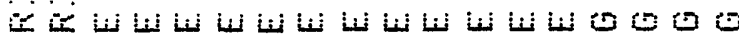

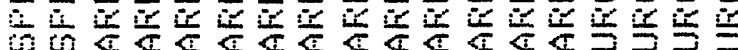

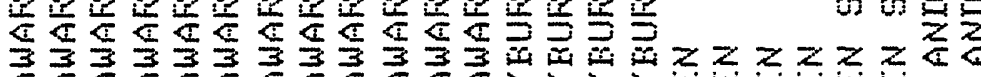

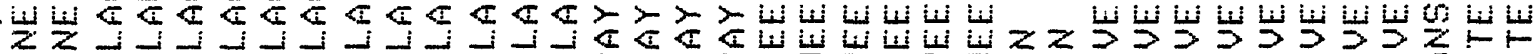

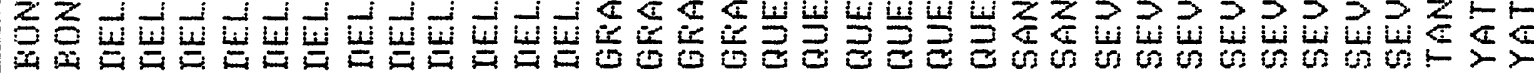

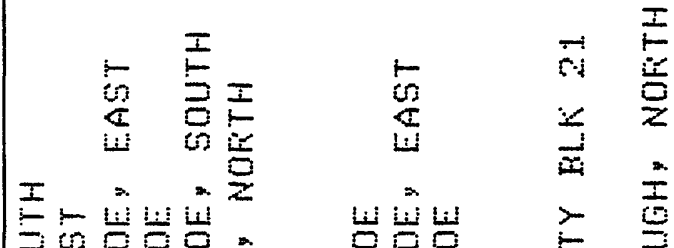<smiles>C#CC#[Ge]</smiles>

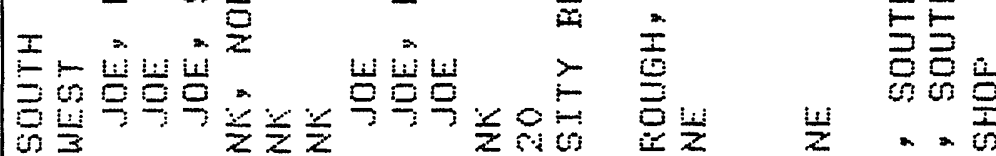

in 4 L

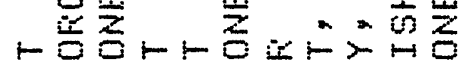

$\frac{i}{6}$

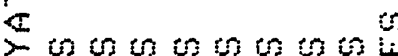

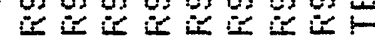

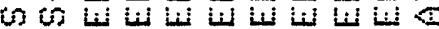
2 . 定 $=4$

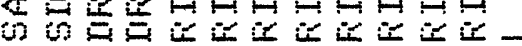




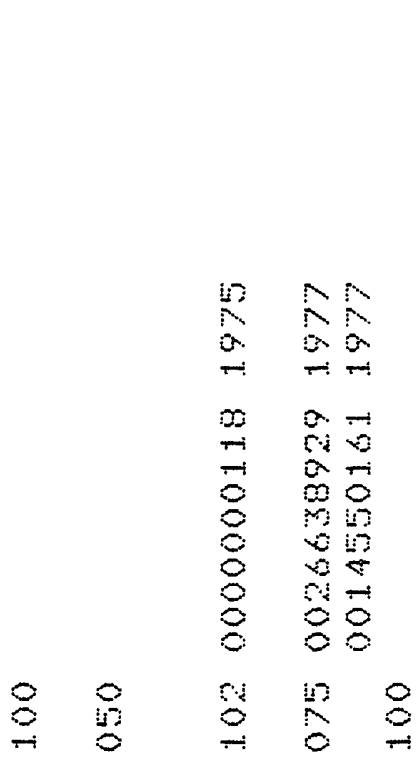

0001250000 अ $\rightarrow$ mo

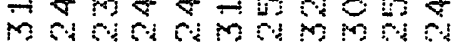
00000000000

an Non

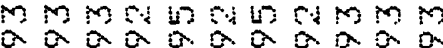
a 2020020200

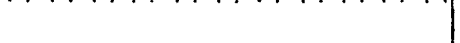

क

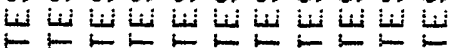

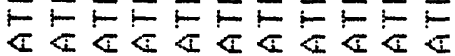
$x>x \leq x \leq x \leq x$

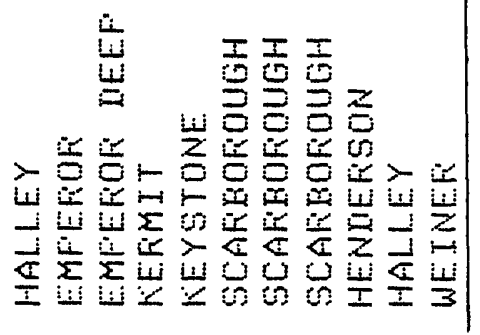

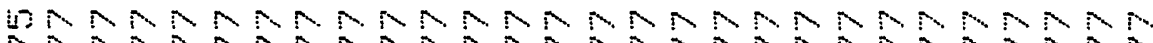

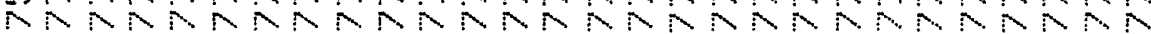
0020002000002000000000000000

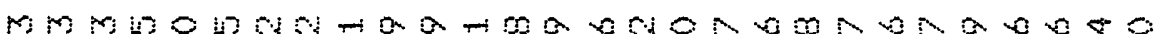

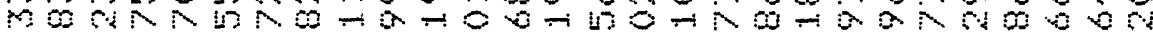
m

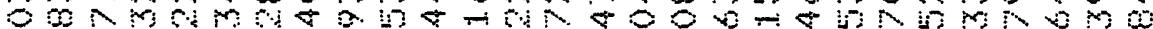

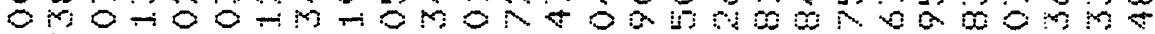

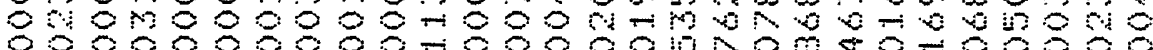

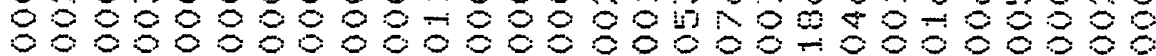

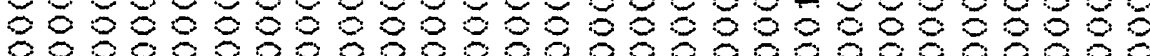

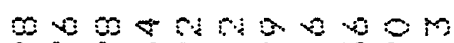
050 a

का

- $00000000 \%$ omo00+10000

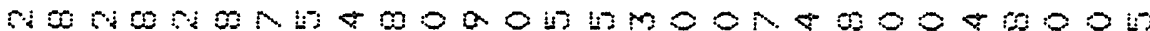

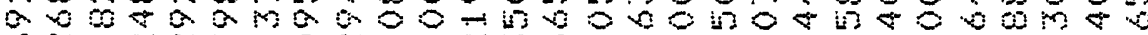
a

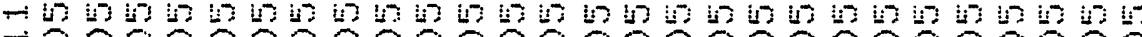
- 00000000000000000000000000

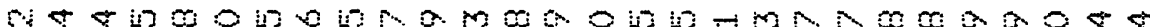
15

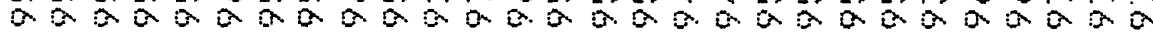

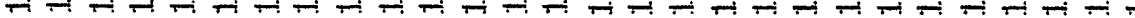

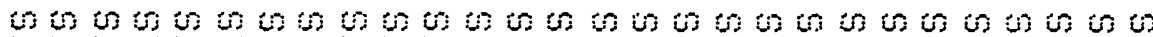

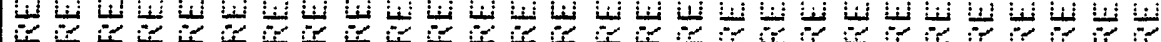

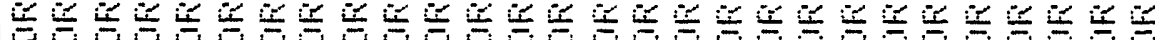

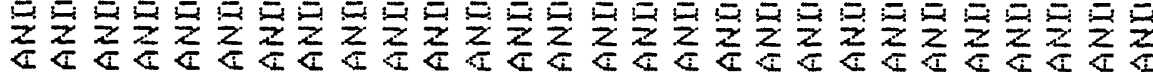

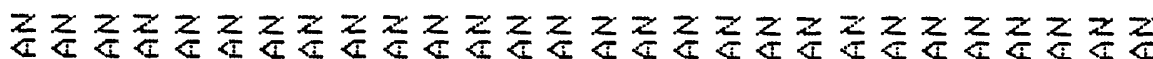

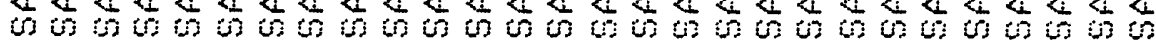

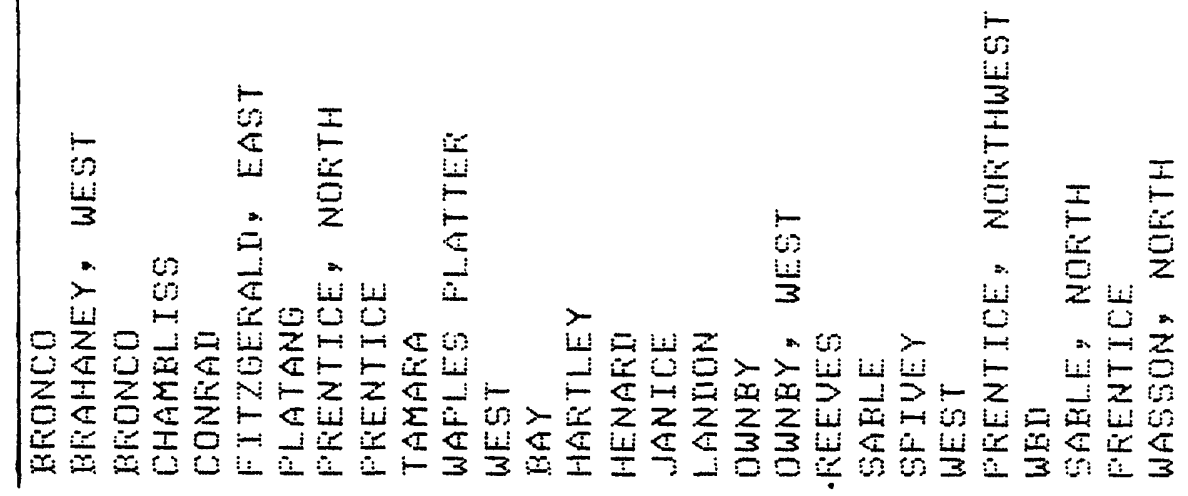


$\mid \begin{array}{ll}5 & \\ 3 & 0 \\ 3 & 0 \\ 3 & 0\end{array}$

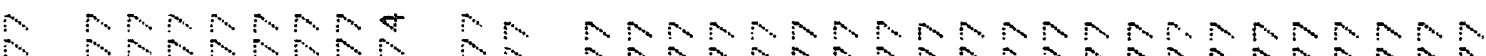

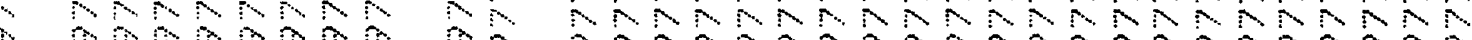
$\rightarrow-i-i+i m i$

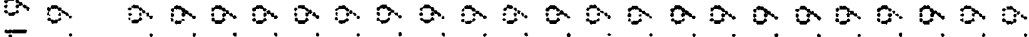
$\infty \quad \infty ; \infty$ iा

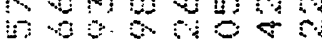
$\therefore \rightarrow \div 300$ $0000 \%$ $0 \div 00 \%$ $\infty m \infty 000$ $00 \div 0+0-0$ 8008080

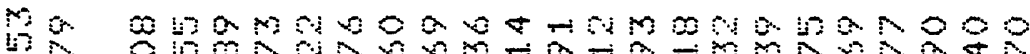

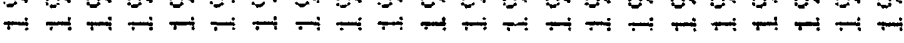
mo कmo iन ㄴ

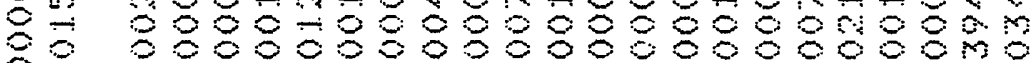

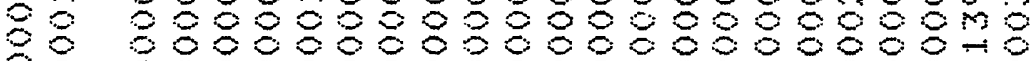

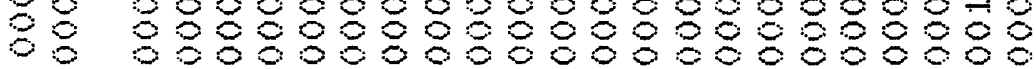

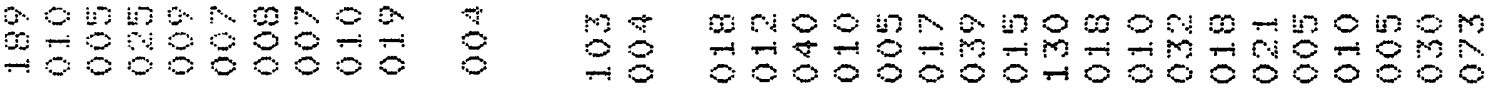

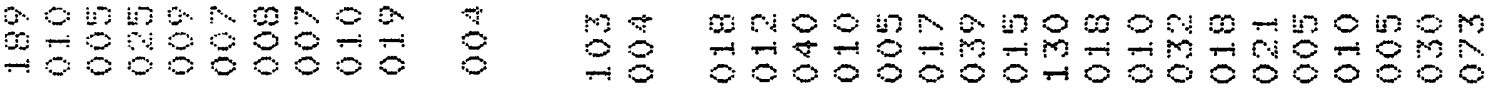

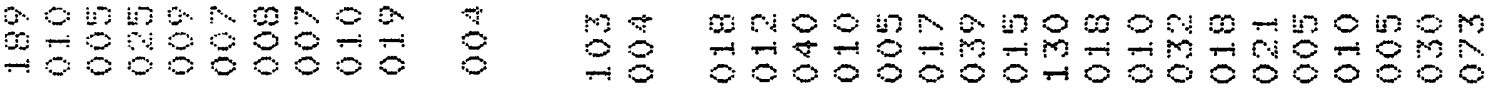
मकम रुम (100000000000000000000000

\%की

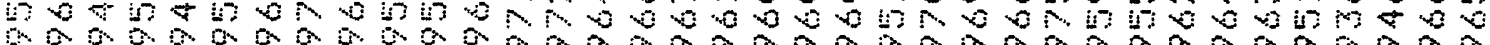

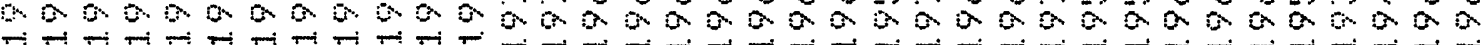

10

6 6

$\stackrel{=}{=}$

-

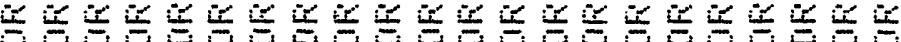

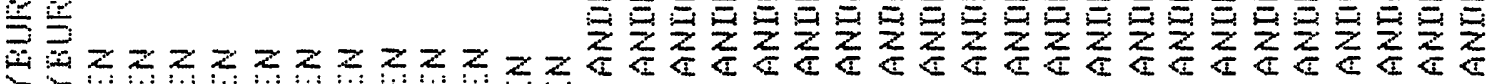

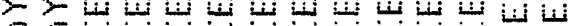

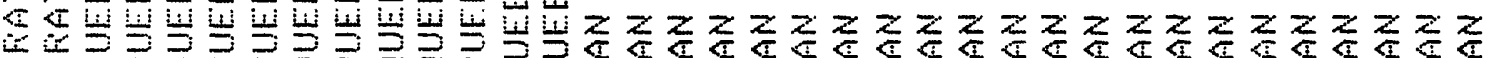

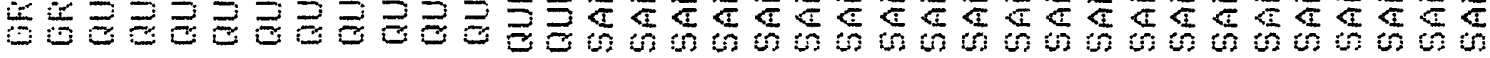

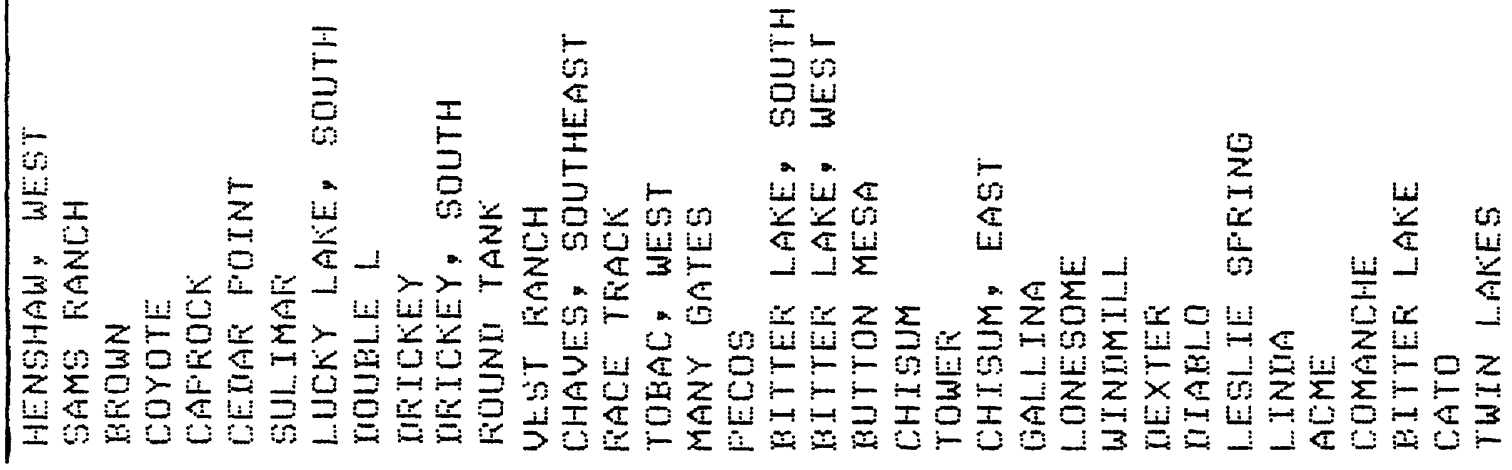




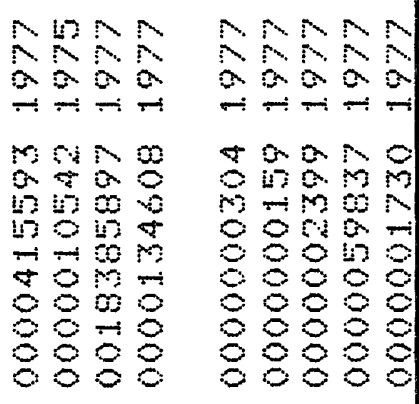

$8 \quad 80$

i क कm

$\begin{array}{ll}0 & 0 \\ 0 & 0 \\ 0 & 0\end{array}$

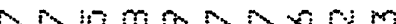
$0000 R+20$ 000000000 두

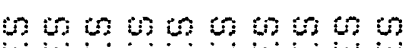

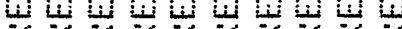

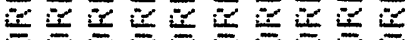

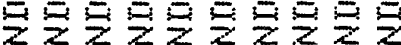

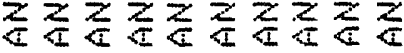

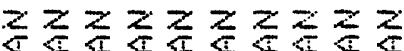
की

O

:

200 -

-

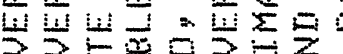

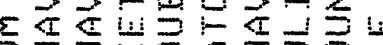

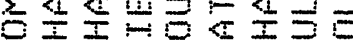

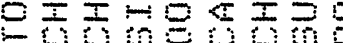

$\frac{8}{8}$

8

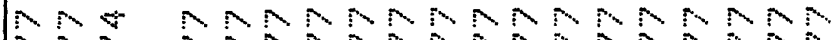

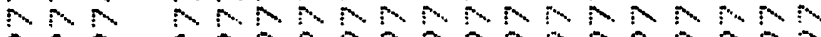

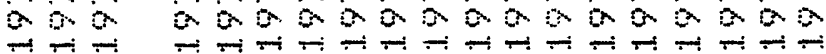
$\infty+\infty$ $-\infty+\infty$ $0 \%$ $0+8$ it: 00 808 868

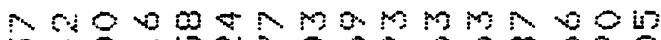
if

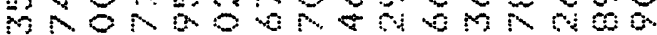

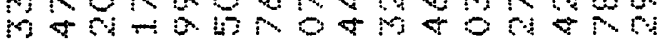
06\%

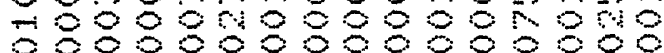

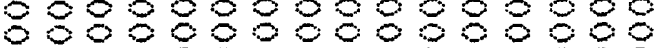

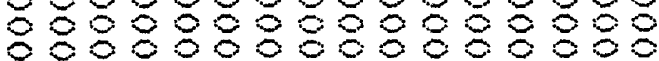

$\infty \infty 0$

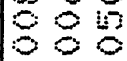

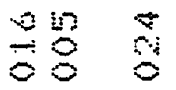
in $\infty<\infty$

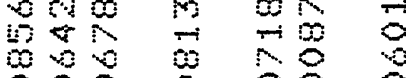
000

8080

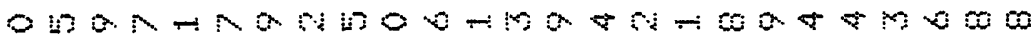

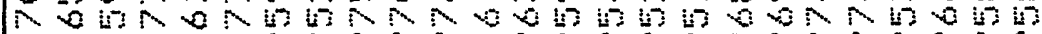

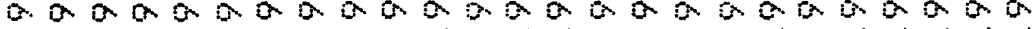

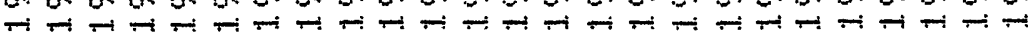

४

6880

$N_{0}$ $\infty 000$ $5 \%$ o. $0: \%$ स 8 063 8883 2808 $\sin 8+\pi 00 \%$ M

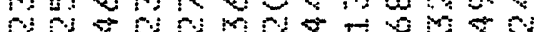
moo

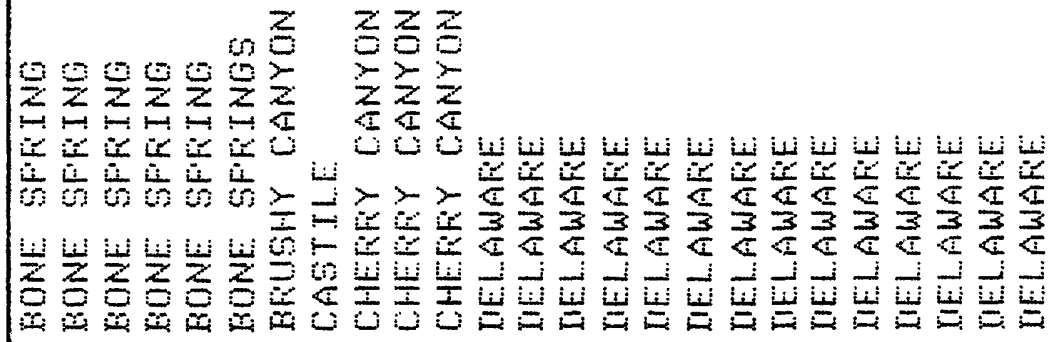

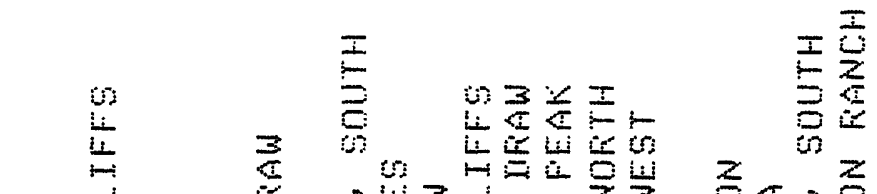

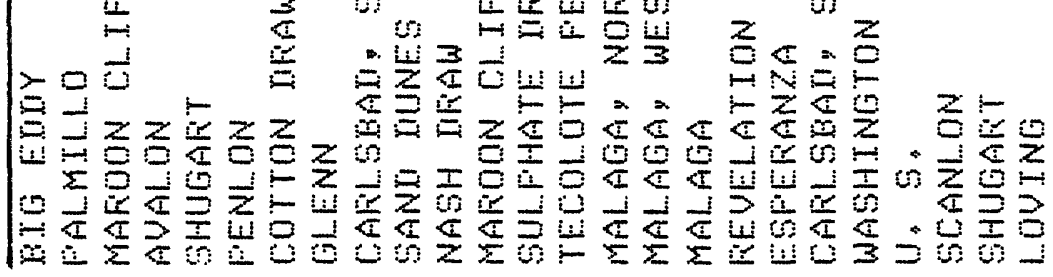


$\begin{array}{lll}6 & 8 & 0 \\ -1 & 0 & 8 \\ 8 & 0 & 8 \\ 8 & 8 & 8\end{array}$

NAR NRE

काष A2

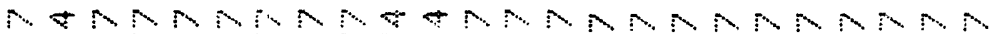

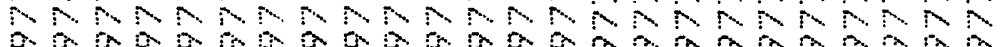

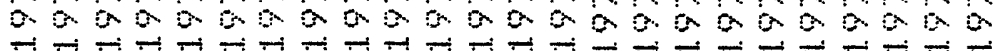

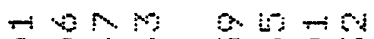

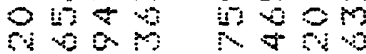

$\sin$

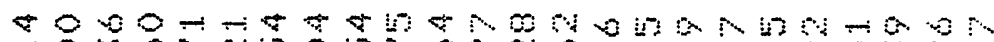

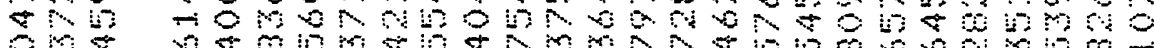
क४

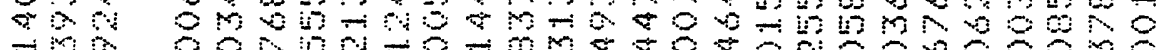

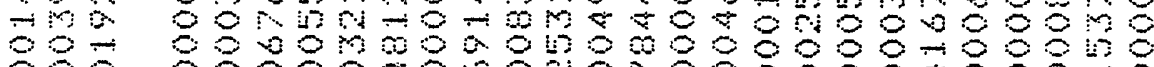
480100 mb $2 m 0$ $60 \%$ का 80888

88

888

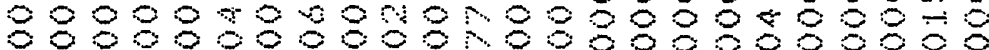
888888888888888888888888

$\rightarrow$ in momono

150 6060

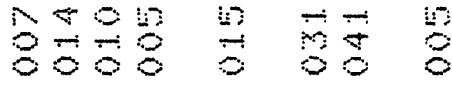

80

8

$\operatorname{mon}$

0 wris

$\rightarrow \cdots$

$\rightarrow$

8

87

in

60
208009

00000

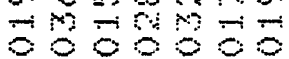
mon म0mminoom

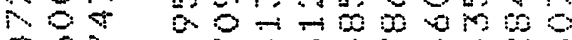

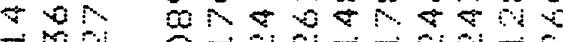
की

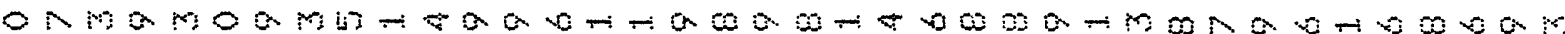
사.

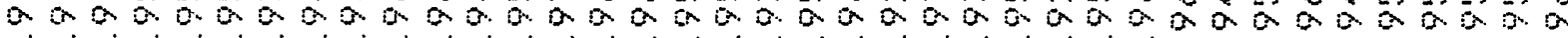

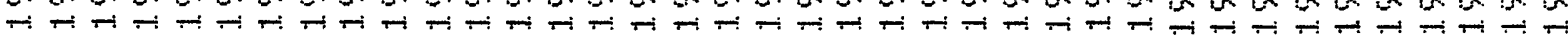

\footnotetext{
to

$\lim _{0}$

(i) $<\div$

$+>$

$\sum z \frac{1}{2}$

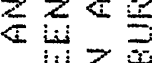

$2 \pm \frac{1}{2}$

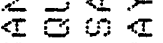

(i)

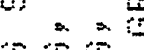

000

$=10$ ir $\sum_{i} \sum_{i}^{\infty} \sum_{i}^{2}$

$z \geq \geq$

$\sum_{i=1}^{2}$

000000000

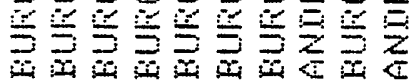

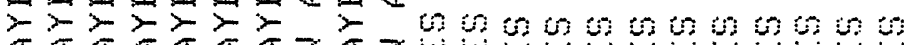

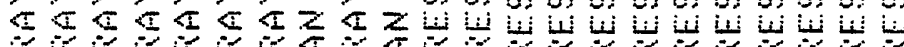

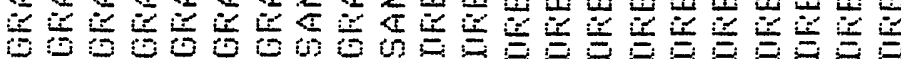

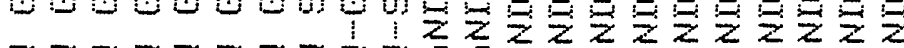

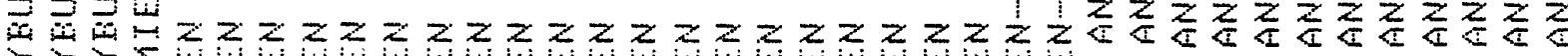
>

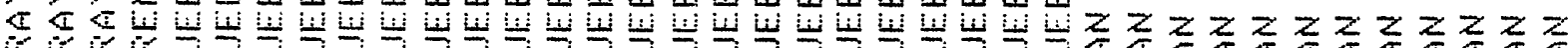

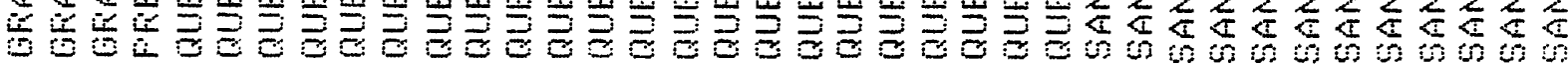

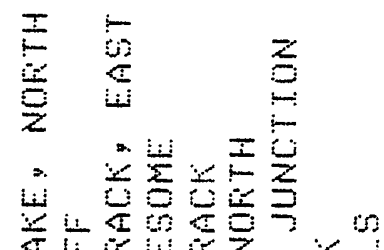

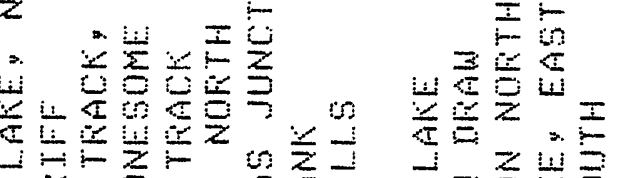

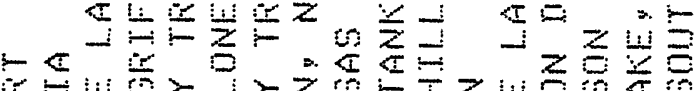

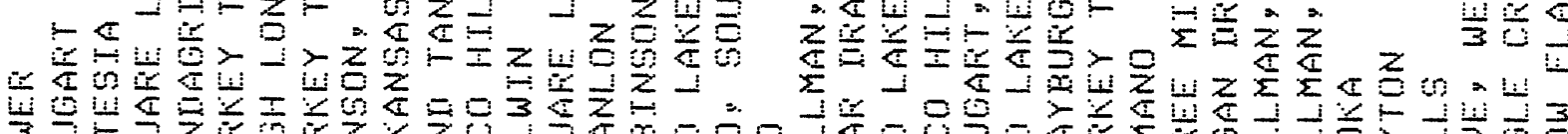

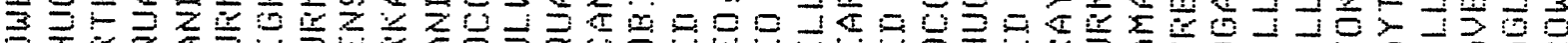
는 


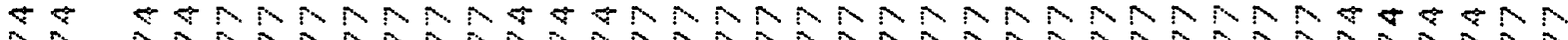

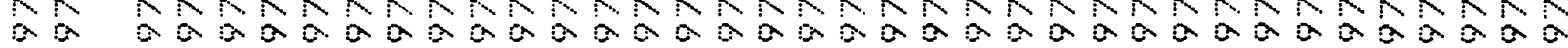

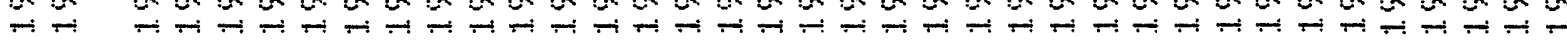

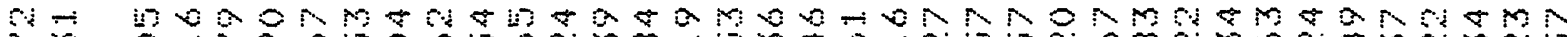
Nm b-1 o m

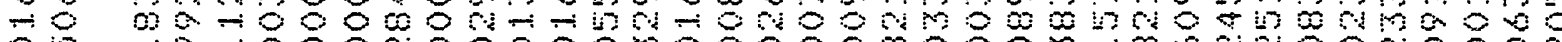

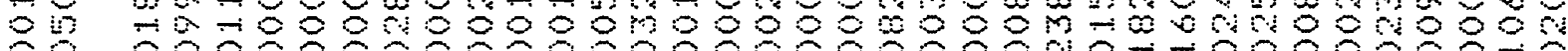

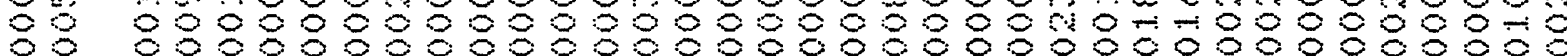

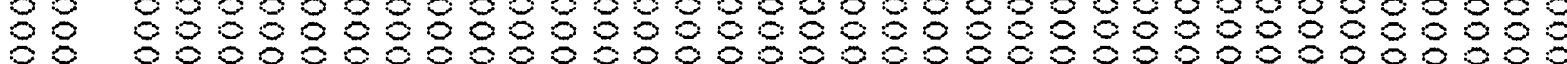

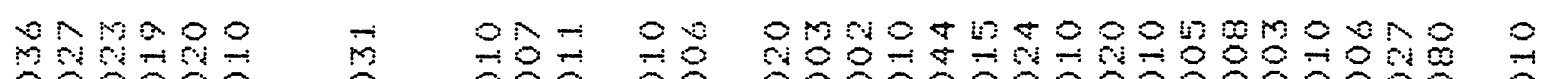

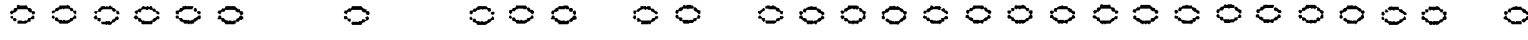

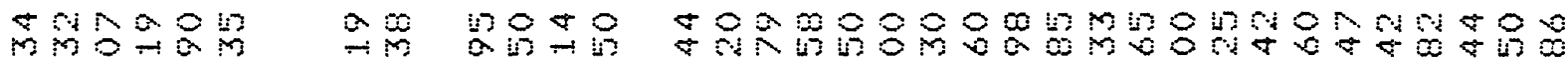

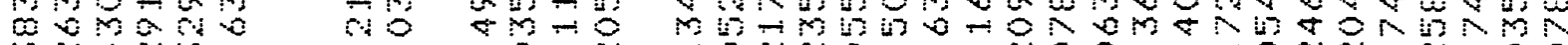

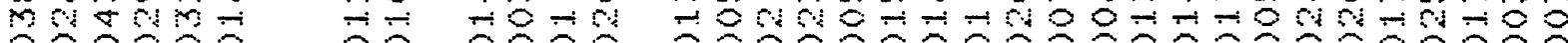

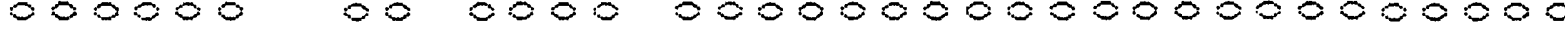

क 15

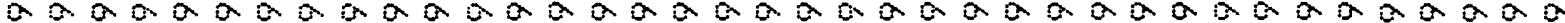

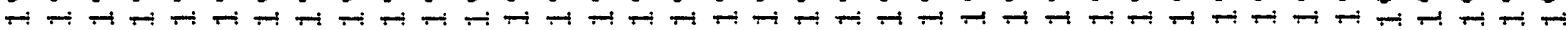
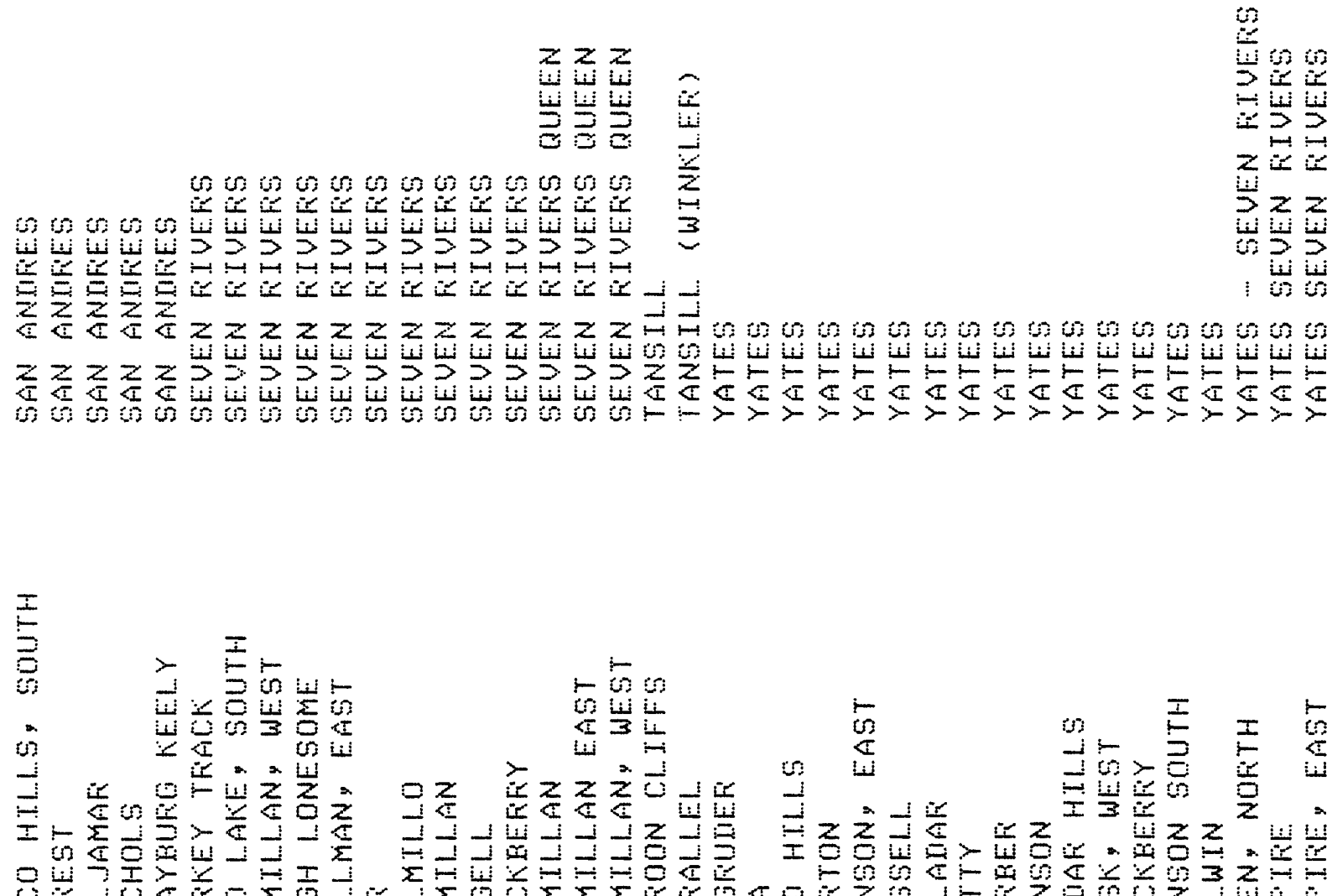

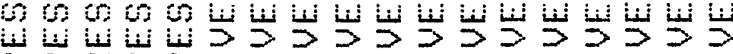

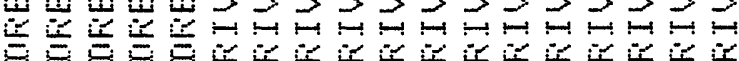

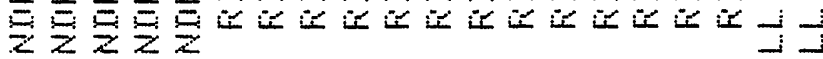

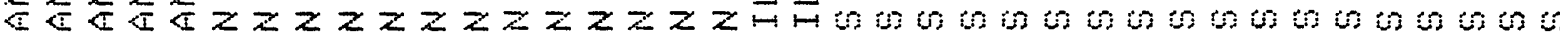

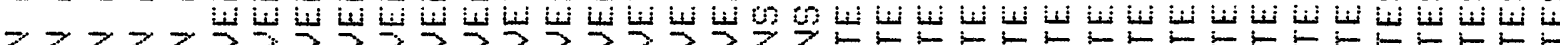

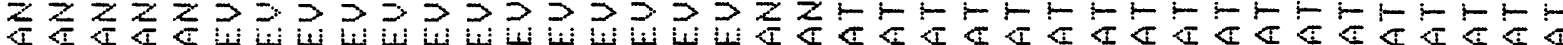

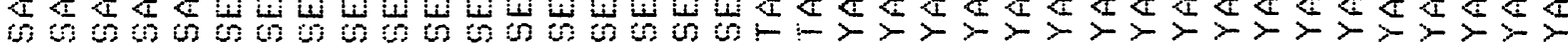

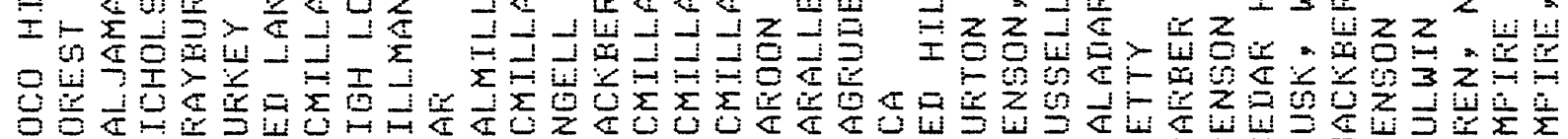
- 


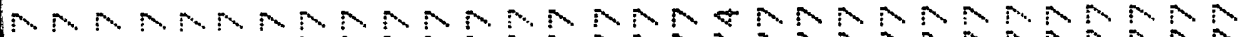

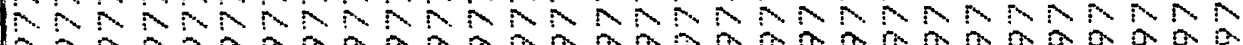

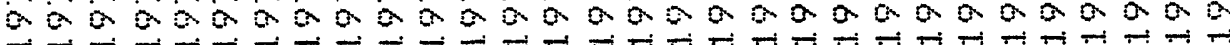

$\prod_{020} \infty 2$

$\rightarrow$

$\infty \infty \infty$

$+\infty \infty$

150

$\pi \div$

No

So

8.6

100

$2 \div 8$

000

in i:-

6

880

$\begin{array}{lll}0 & 0 & 0 \\ 0 & 0 & 0\end{array}$

$\rightarrow$ - 00 No

- in $\mathrm{C}$ \%

a 0000

-1斤H

os 6

10

$5 \frac{1.5}{5}$

$\because=$

$2 z$

3

i.i i.i.

6 6

os en

证过0 00

匹

$>>\dot{x}>>$
ن

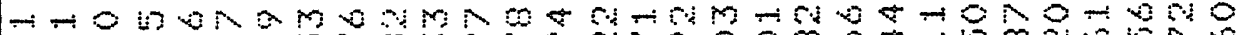
म 0- न-

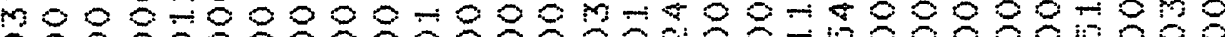

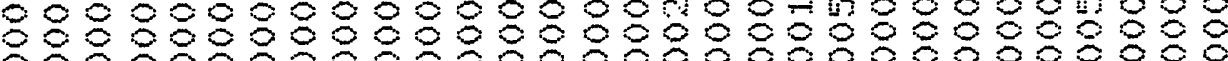

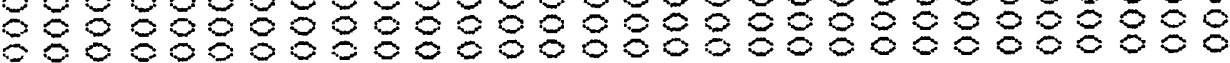

O

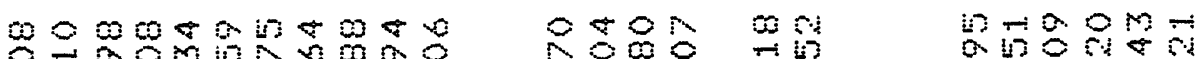
연

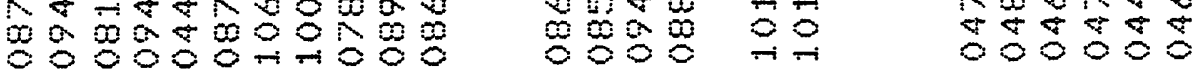

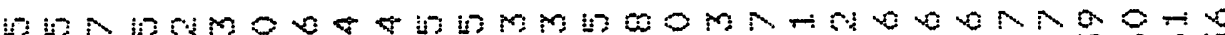

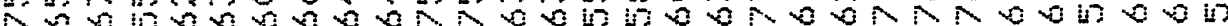

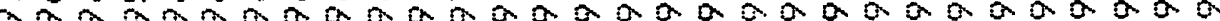

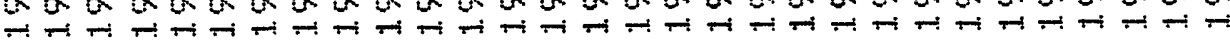

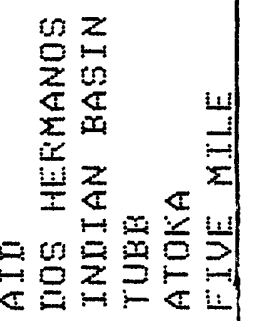

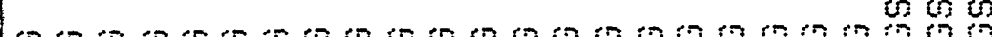

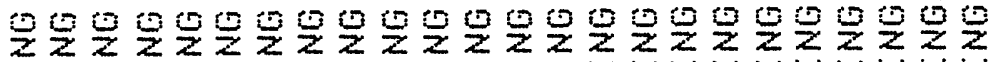

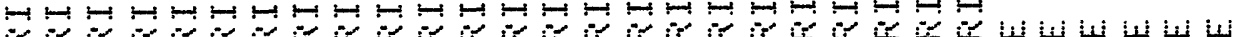

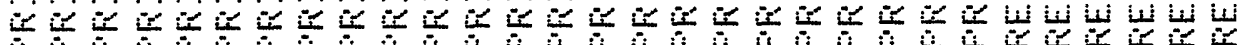

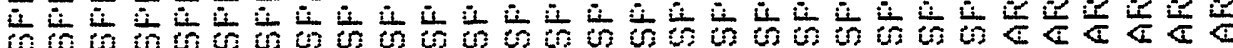

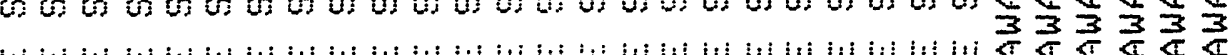

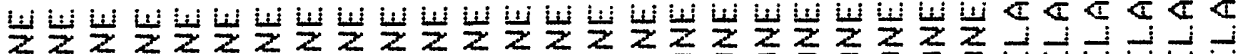

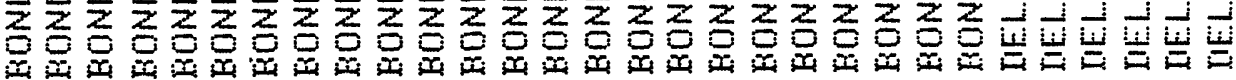

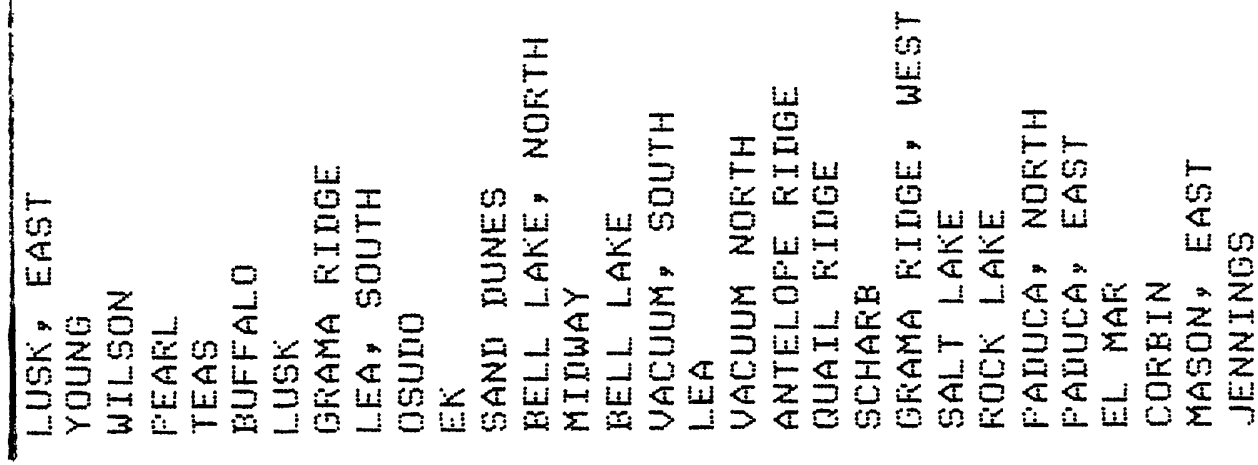




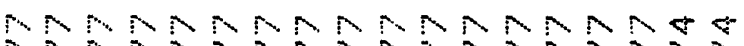
20

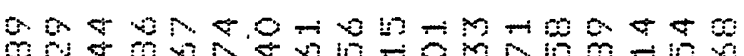
60

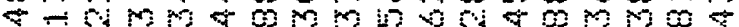
स- m

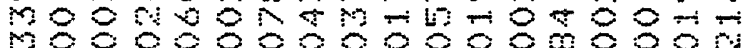

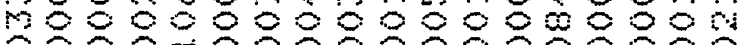

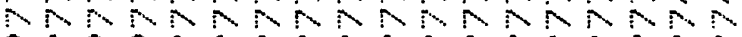

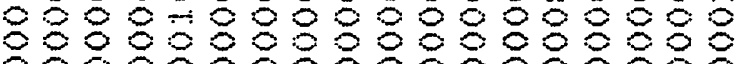

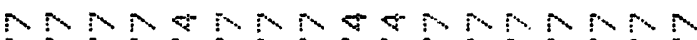

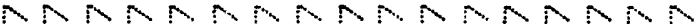

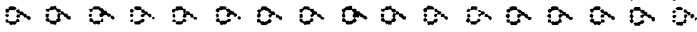

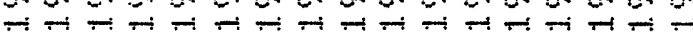

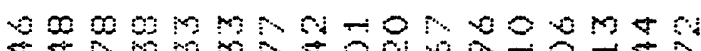
b-60 की

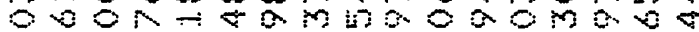
8 -60\%

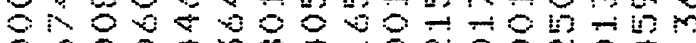
$6000 \%$ m. 606유

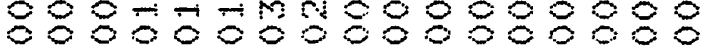

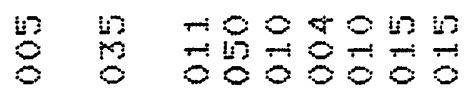
웅융윰형뭉 100000000000 1) 1000 Tio

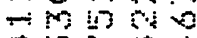
4308

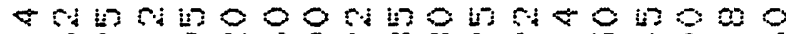

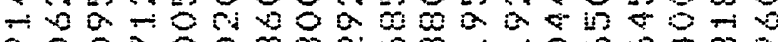

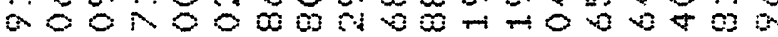

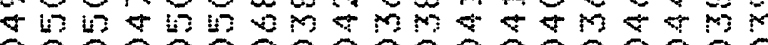

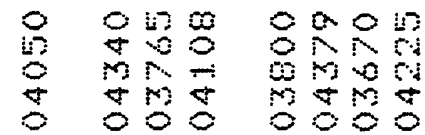

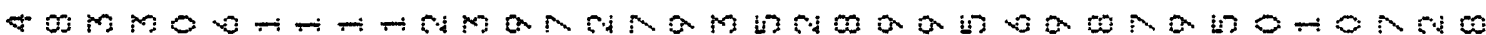

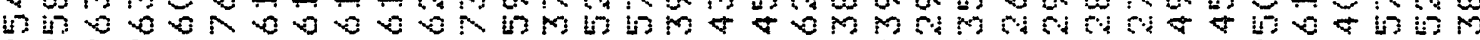
200000000000000000000000000000000000 -

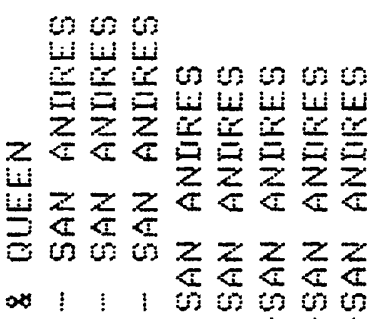

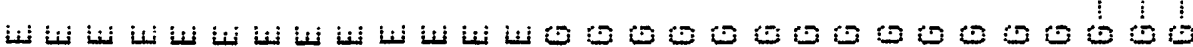

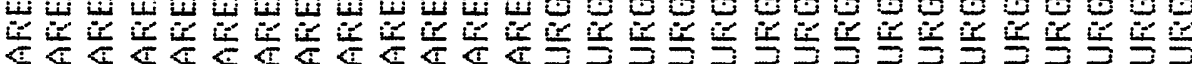

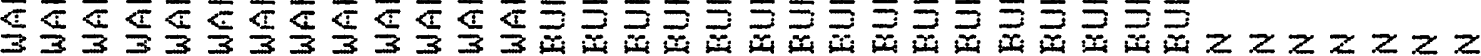

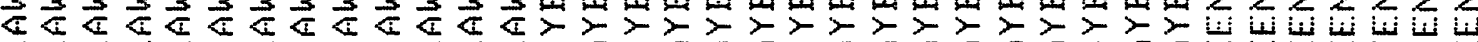

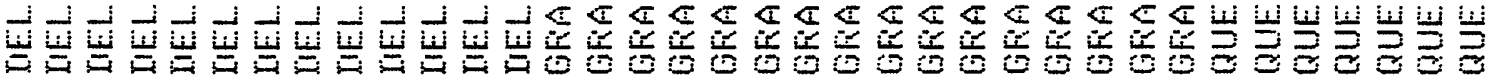

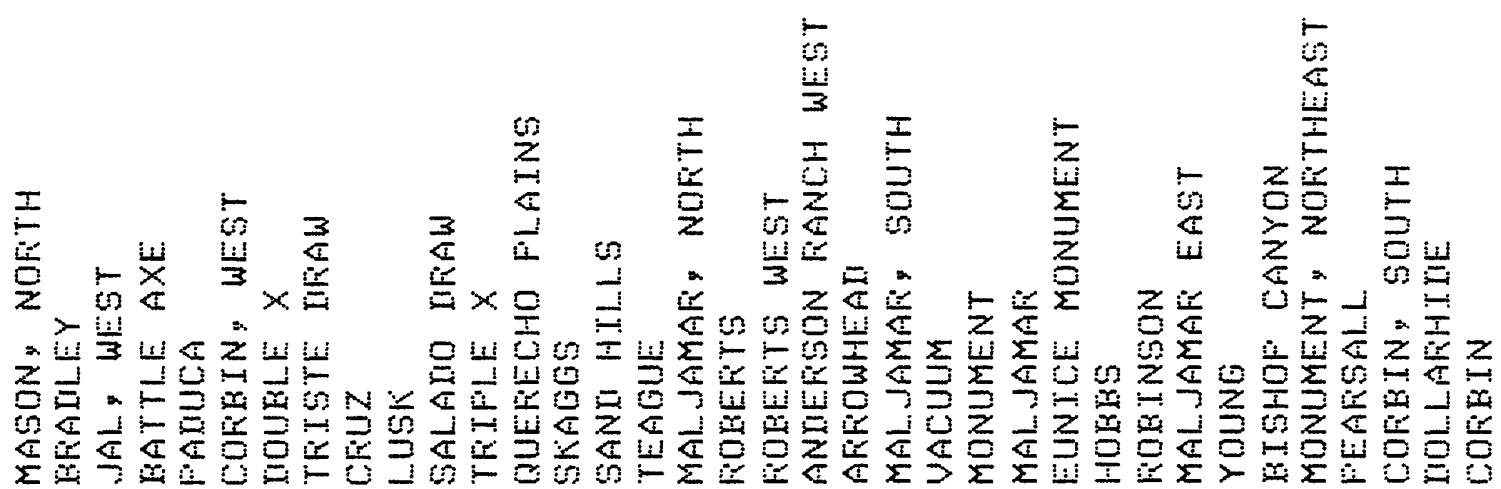


$4 \pi \sin$ som $\infty 20 \div-\infty$

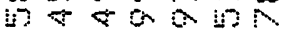
किष्ठक्ठ

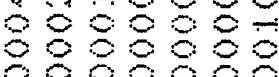

NA

$\hat{2} \hat{2}$

$\rightarrow \rightarrow+$

aro

80

$8+8$

$\infty \infty$

20

838

क्षम00 onow00 mind

$\infty<\mathrm{O}-\mathrm{mm}$

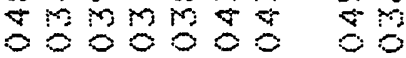

205

iो

का

$\rightarrow \&$

$\rightarrow 10$

$0 \div 8$

880

$\infty$
08
58
0
NANRTARTOR

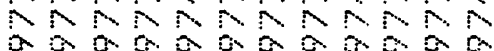

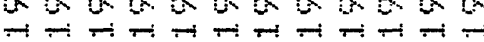

$000 \mathrm{mon} 00 \mathrm{mor}$ TR HMOQRRO060

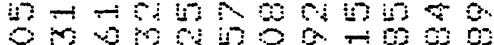
०- के कीनिका

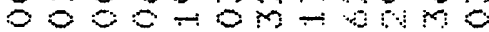
०06000001000

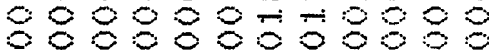
88.888888888

00

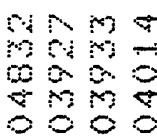

0
8
8
8

WNMNANAN $N M N D N D N D$ 200000000

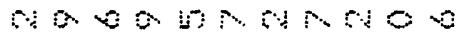
$\rightarrow \sin m \& \operatorname{mon}$

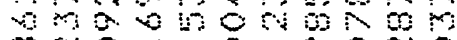
$20 \div \div-300 \%$

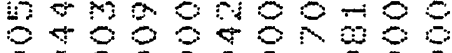
$\% 00000 \div 080$

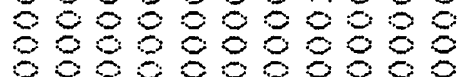

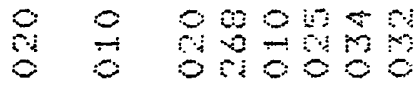

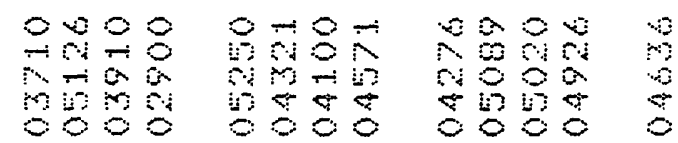

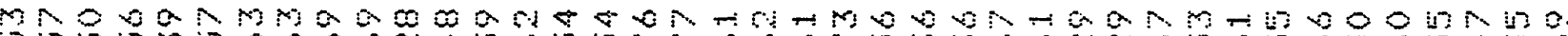

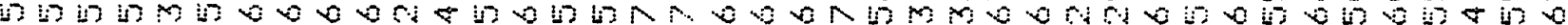
a

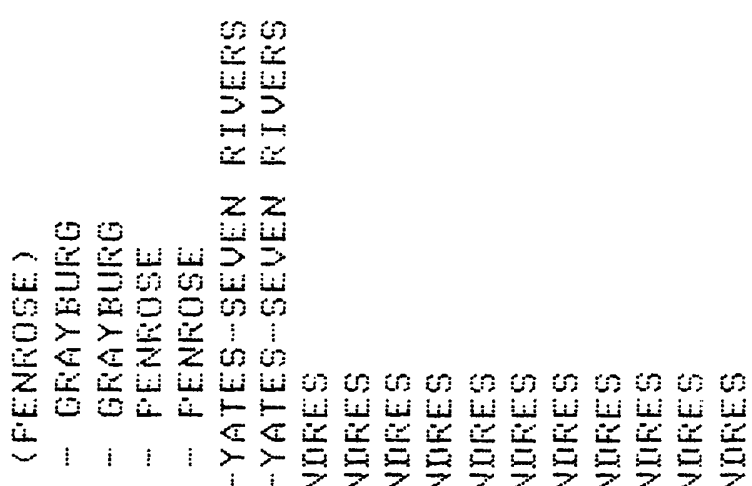

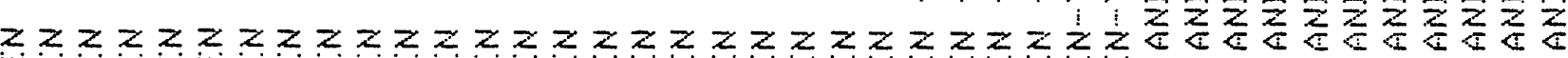

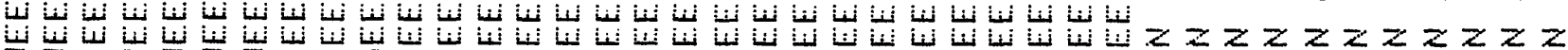

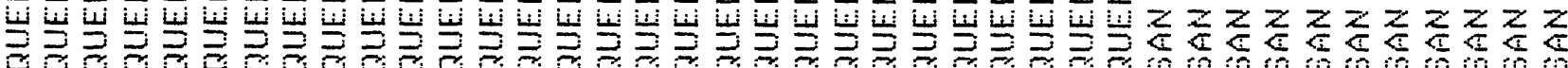

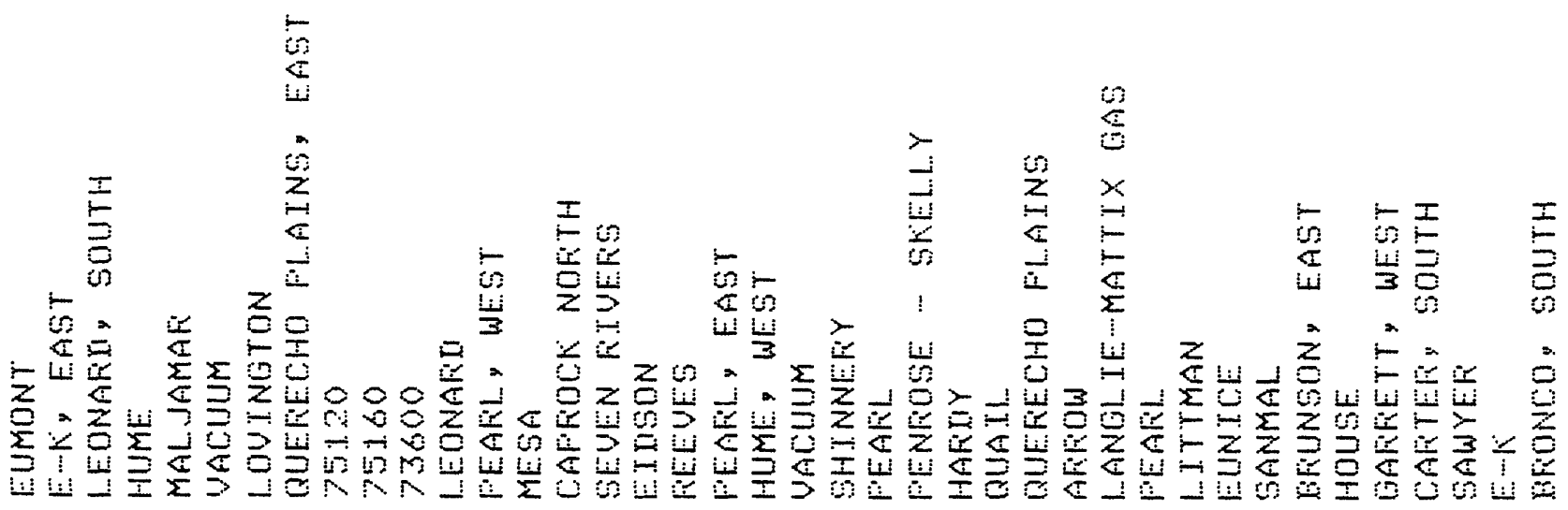




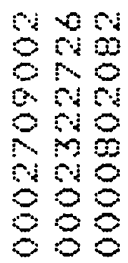

8
8
6
8
8

NRNRNRRNRNR $\alpha 20 \alpha 20000202$

$\mathrm{NSND}$

NhNS $\infty 2 \infty 200$ $\rightarrow-i \rightarrow-i$

$+00 m 0:$

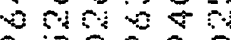
2 it 020 $\infty-4012$ $6+02 i$ $2+80128$ 1.858

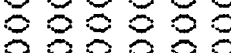

- M+

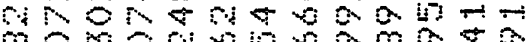

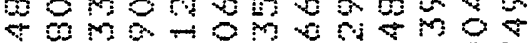

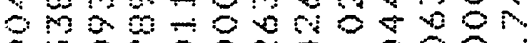

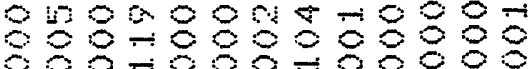

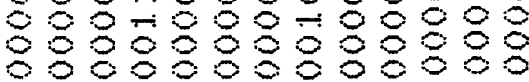

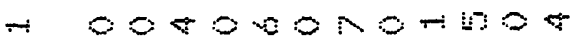

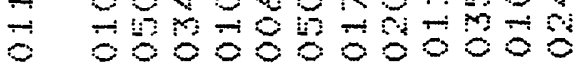

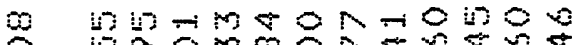

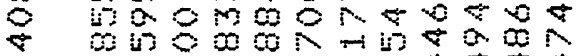
\&

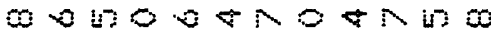
65

\& $200+\infty \infty \infty+110$ $00+<+\infty<+100+12$ काषनक षष
४ 20020000000000

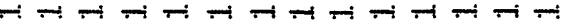

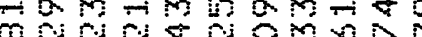

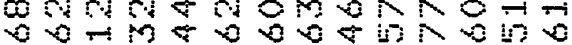
और -00-0000-0000m

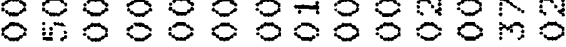

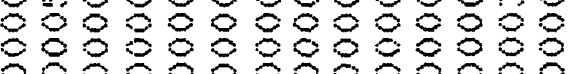

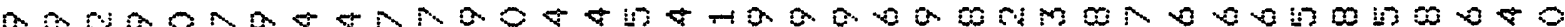

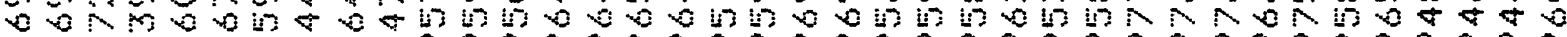

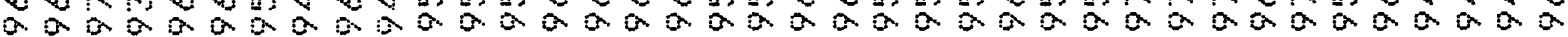

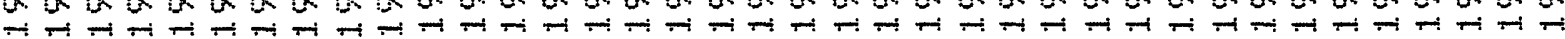

की th

计证证证

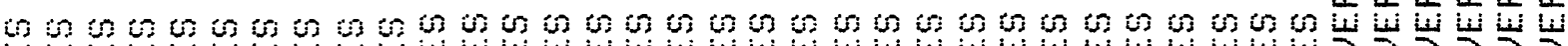

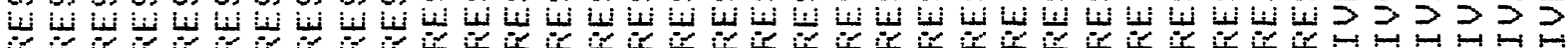

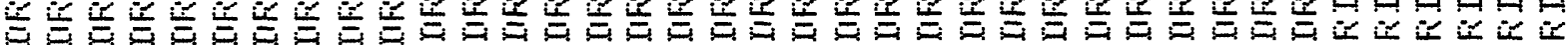

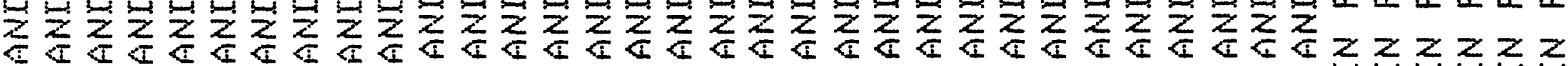

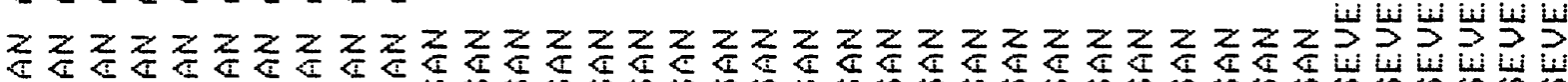

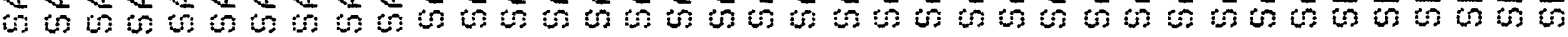

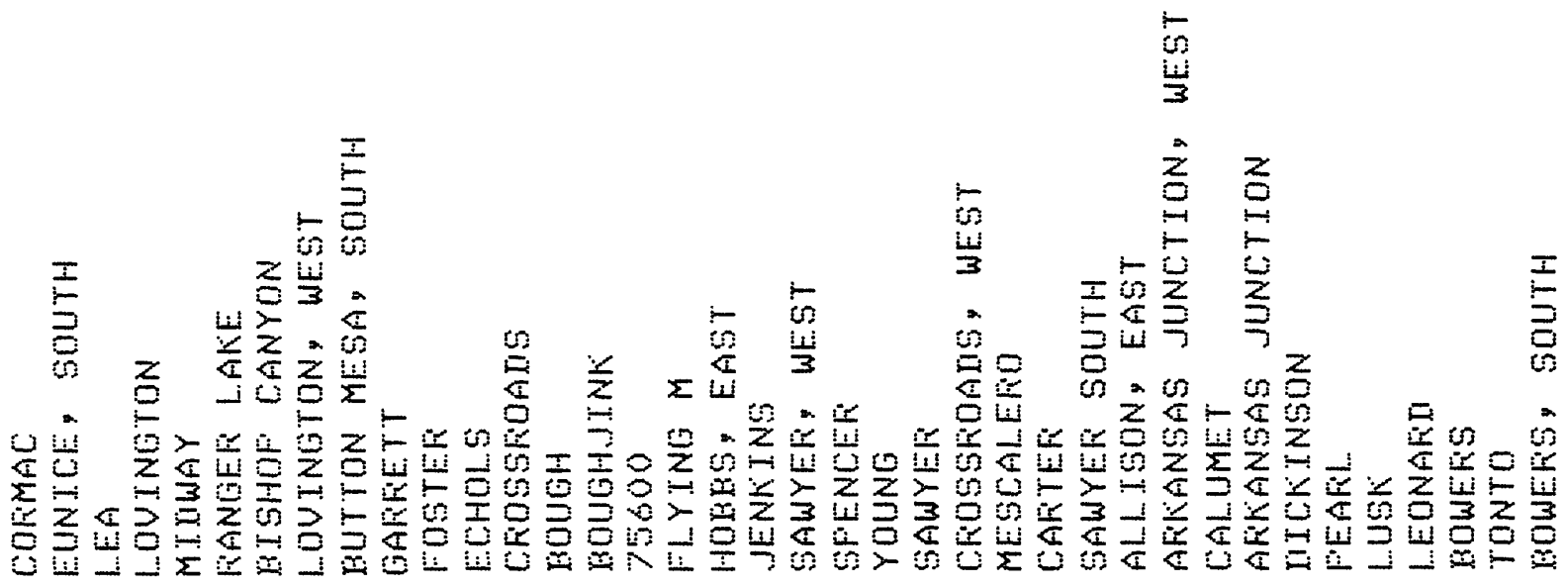


8

$\sum_{\rightarrow \infty}^{\infty} \infty_{i \infty}^{\infty}$

$12 m o$

क

अ is

$\therefore \quad 20$

$0 \quad 00$

803

630

$+200$

एक

i2 $00 m 0$

$m$ \&

00000

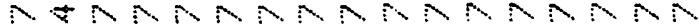

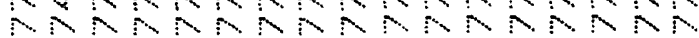

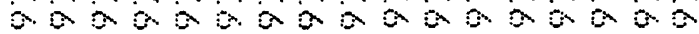

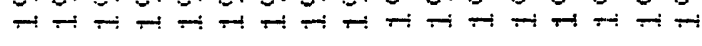

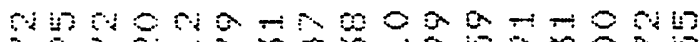

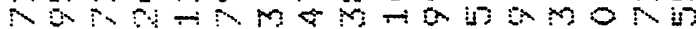

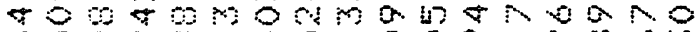

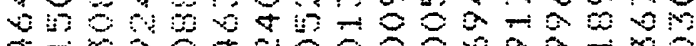
म․

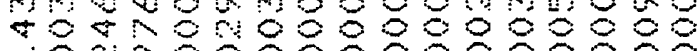

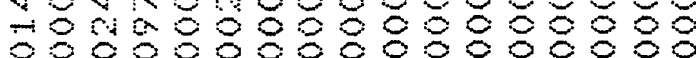

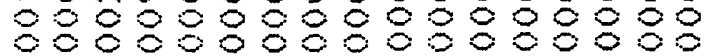
팡 20000 $m m m$ 00000 0 00 0 obo 00 $\begin{array}{ll}0 \\ 0 \\ 0 & 8 \\ 0 & 8 \\ -1 & 8\end{array}$

NNSWN NDTS N 3020000 $200+0 \%$ aक $00 \div-\infty m+$ $00-\infty \mathrm{s}$ की० $088 \div 60$ 6866015 6888880

$\begin{array}{ll}6 & 5 \\ 8 & 8 \\ 8 & 8 \\ 8 & 8\end{array}$

NST $\infty 200$ $\lambda-m \infty m$ $00 \infty 11-0$ $\infty$ is 0 क n $m a+\infty$ 000000 $\because 868$ 150 688880 85868

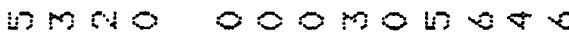

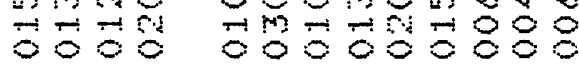

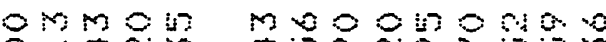
-

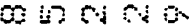

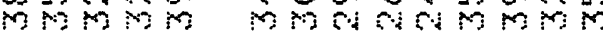

0000000000000

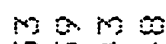
15.

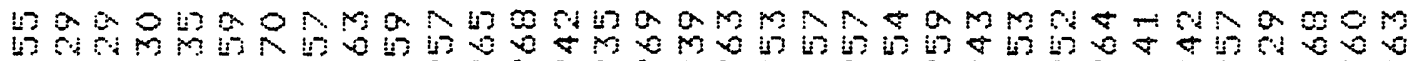

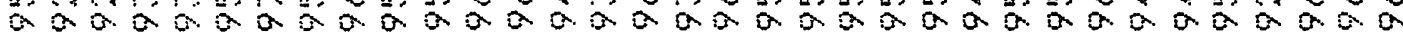

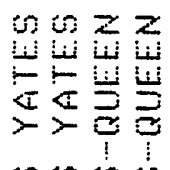

in on on on on on in

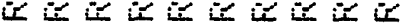

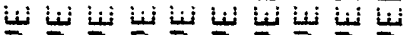
3232353232

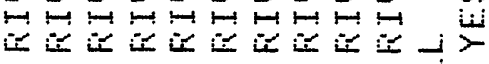

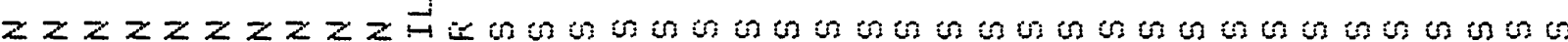

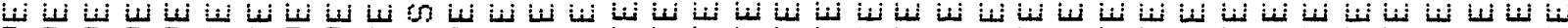

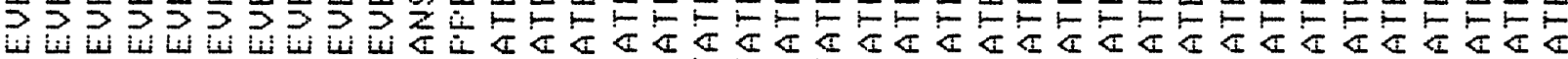

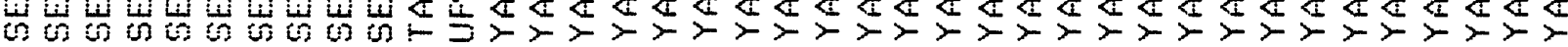

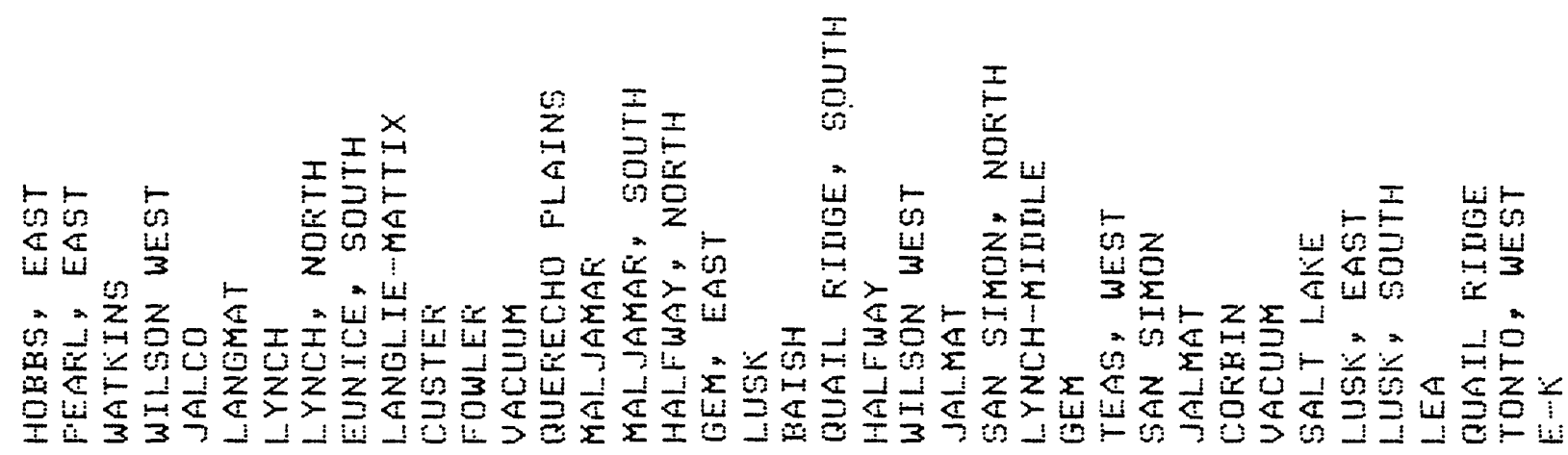


$\begin{array}{ll}0 & 0 \\ 8 & 0 \\ 8 & 0 \\ 8 & 0 \\ 8 & 0\end{array}$

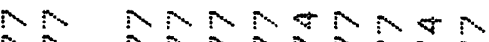

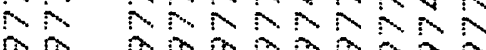
an ana 20202

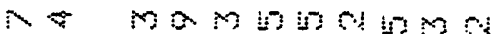
00 mom 1000 क 45 मी $\infty \div+\infty 00 \% 0 \%$

तis 000 का

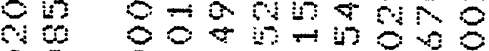

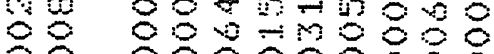
68 8806808888

궁 ती -6 की० की

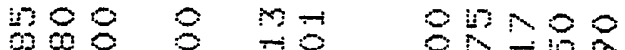

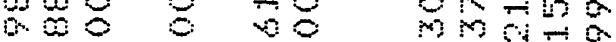

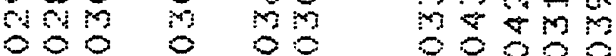

$\begin{array}{ll}2 & 0 \\ 0 & 0 \\ -1 & 0 \\ 0 & 0\end{array}$

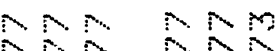

Sर

$20 a 00$

$+\div$

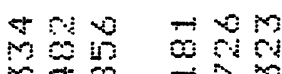

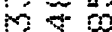

$0 \div 0$

$\div \oplus$

$60 \mathrm{~m} 0$

608088

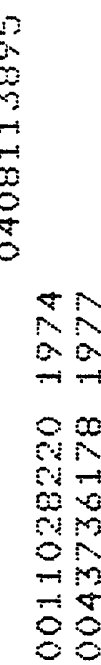

ir $0000 \mathrm{~m} 000 \mathrm{n}$

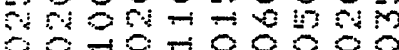

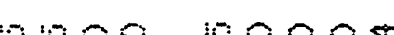
:1200 $00 \% \hat{0}$ 5 i50 0000100

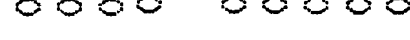

Nली

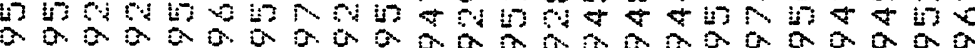

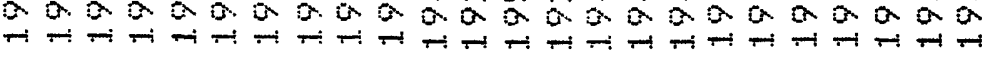

$00000 \frac{1}{0}$

$\because 25$

-

$333 \div \div \div=10=$

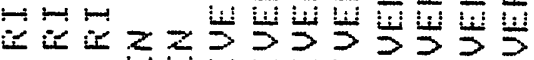

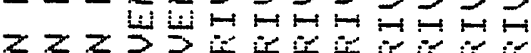

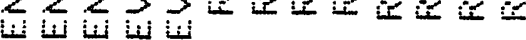

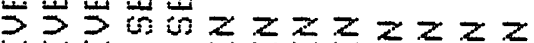

iv

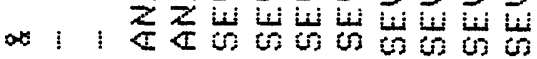

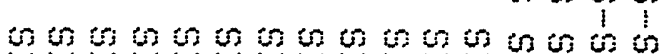

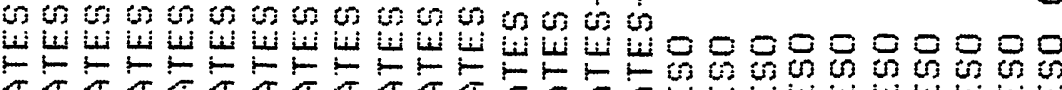

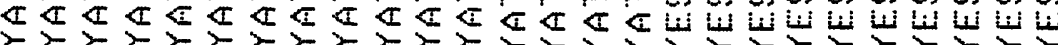

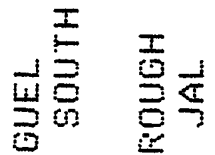

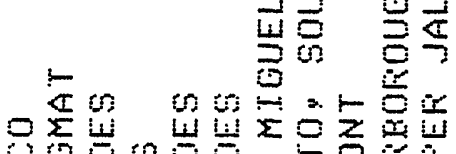

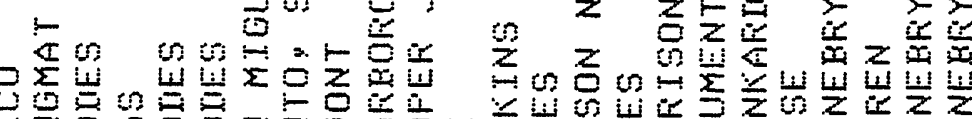

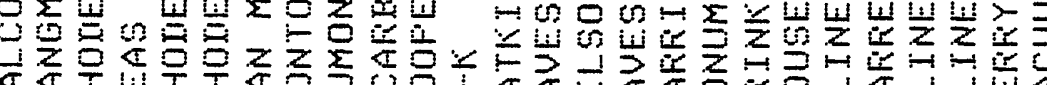

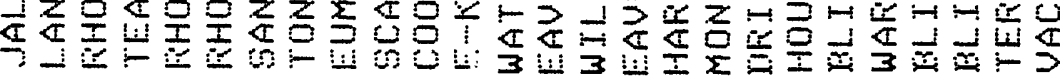

WRNRRN

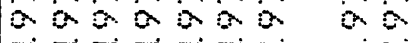
$\rightarrow \rightarrow+\pi \rightarrow+T \rightarrow$ $\infty \infty-10+200$ नकी 6060-00 ㅇㅇㅇㅎㅇ

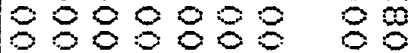

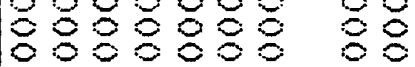
움

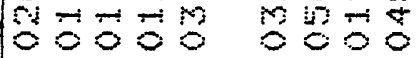
$\leftarrow 0+\rightarrow \infty 00$ 1200 in 10 in

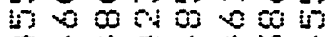
दठ

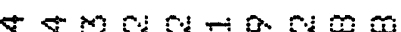
60000 तो 2000020000

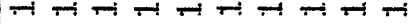

पु की

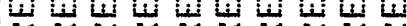

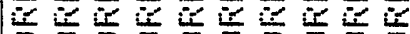

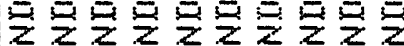

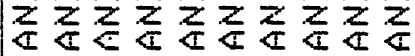

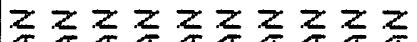

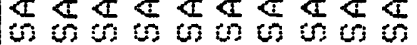

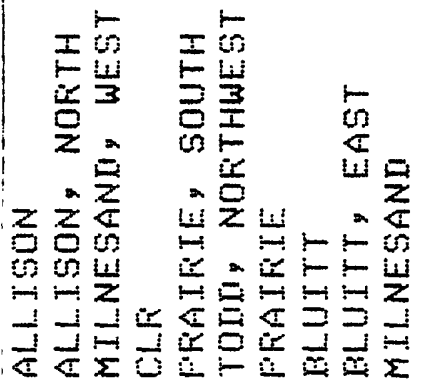




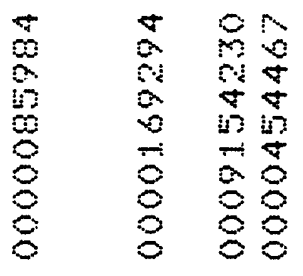

กิ

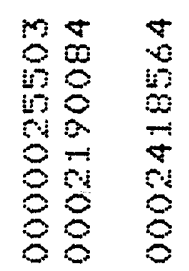

영

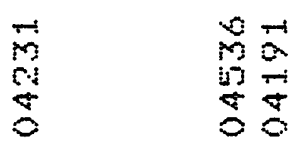

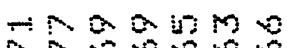

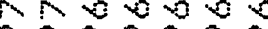

2020202

这

$=$ ir

$\leftarrow \quad$

可.

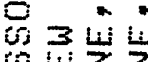

녈종종

की

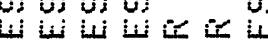

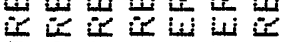
司是上5

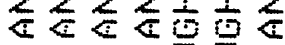

$z z z z$ 死

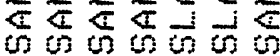

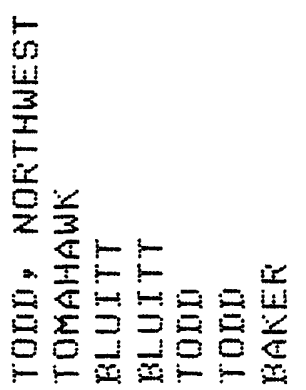


Table 4.--Cumulative oil and gas production for selected stratigraphic units from the Permian Basin of west Texas and southeastern New Mexico

\begin{tabular}{|c|c|c|c|}
\hline $\begin{array}{l}\text { Producing } \\
\text { Formation }\end{array}$ & of Pools & $\begin{array}{l}\text { Cumulative } \\
\text { oil production }{ }^{2} \\
\text { (bbls) }\end{array}$ & $\begin{array}{l}\text { Cumulative } \\
\text { gas production } \\
\text { (Mcf) }\end{array}$ \\
\hline Bone Spring Ls. & 36 & $10,836,946$ & 26,107 \\
\hline Capitan Fm. & 0 & 0 & 0 \\
\hline Castile Fm. & 10 & 177,449 & 0 \\
\hline Delaware Mountain Gp. ${ }^{4}$ & 228 & $147,838,929$ & 444,186 \\
\hline Goat Seep Ls. & 0 & 0 & 0 \\
\hline Grayburg Fm. & 173 & $3,257,310,434$ & $17,265,940$ \\
\hline Queen Fm. & 179 & $527,719,866$ & $1,254,017,480$ \\
\hline Rustler Fm. & 5 & $1,019,685$ & 0 \\
\hline San Andres Ls. & 435 & $3,605,905,136$ & $36,433,294$ \\
\hline Seven Rivers Fm. & 91 & $921,401,640$ & 823,028 \\
\hline Tansil1 $\mathrm{Fm}$. & 12 & 229,717 & 0 \\
\hline Victorio Peak Ls. & 0 & 0 & 0 \\
\hline Yates $\mathrm{Fm}$. & 159 & $609,064,948$ & $1,710,628,578$ \\
\hline Yeso Fm. & 15 & $202,411,700$ & $803,437,919$ \\
\hline $\begin{array}{l}\text { Total } \\
\text { all units }\end{array}$ & 1343 & $9,283,916,450$ & $3,823,076,532$ \\
\hline
\end{tabular}

1 Data from Table 3, Petroleum Data System (PDS), University of $0 \mathrm{klahoma}$. Data may not be complete as it includes only those pools and production data reported through the PDS system.

2 Most data current through 1977. Some fields only reported through earlier years, however; see Table 3 for reporting years, by field or pool.

3 Most data current through 1977 but some fields may be reported only through earlier years.

4 Includes production from the Bell Canyon, Cherry Canyon, and Brushy Canyon Formations and the Lamar Ls. 


\section{Bibliography of the Permian Basin and}

Related Areas

Achauer, C. W., 1969, Origin of Capitan formation, Guadalupe Mountains, New Mexico and Texas: AAPG Bulletin, v. 53, p. 2314-2323.

1971, Origin of Capitan formation, Guadalupe Mountains, New Mexico and

Texas: Reply: AAPG Bulletin, v. 55, p. 313-315.

Adams, J. E., 1930, Origin of oil and its reservoir in Yates pool, Pecos County, Texas: AAPG Bulletin, v. 14, no. 6, p. 705-717.

1935, Upper Permian stratigraphy of west Texas Permian basin: AAPG

Bulletin, v. 19, p. 1010-1022.

1936, Oil pool of open reservoir type: AAPG Bulletin, v. 20, p. 780796.

1944, Upper Permian Ochoa series of Delaware Basin, west Texas and southeast New Mexico: AAPG Bulletin, v. 28, p. 1596-1625.

1965, Stratigraphic-tectonic development of Delaware Basin: AAPG

Bulletin, v. 49, p. 2140-2148.

1967, Semi-cyclicity in the Castile Evaporite, in Elam, J. G., and

Chuber, S., eds., Cyclic sedimentation in the Permian Basin: West Texas Geological Society Symposium, p. 197-203.

Adams, J. E., and Frenzel, H. N., 1950, Capitan barrier reef, Texas and New Mexico: Journal of Geology, v. 58, p. 289-312.

Adams, J. E., Frenzel, H. N., Rhodes, M. L., and Johnson, D. P., 1951, Starved Pennsylvanian Midland Basin: AAPG Bulletin, V. 35, p. 2600-2607.

Adams, J. E., and Rhodes, M. L., 1960, Dolomitization by seepage refluxion: AAPG Bulletin, v. 44, p. 1912-1920.

and others, 1939, Standard Permian section of North America: AAPG Bulletin, v. 23, p. 1673-1681.

Adams, S. S., 1967, Bromine in the Salado Formation, Carlsbad potash district, New Mexico: Cambridge, Harvard University, unpublished $\mathrm{Ph}$. D. dissertation, 202 p.

1970, Ore controls, Carlsbad potash district, southeast New Mexico, in Third Symposium on Salt: Northern Ohio Geological Society, v. 1, p. 120152.

Anderson, R. Y., Dean, W. E., Kirkland, D. W., and Snider, H. I., 1972, Permian Castile varved evaporite sequence, west Texas and New Mexico: Geological Society of America Bulletin, v. 83, p. 59-86.

Anderson, R. Y., and Kirkland, D. W., 1966, Intrabasin varve correlation: Geological Society of America Bulletin, v. 77, p. 241-256.

1979, Dissolution of salt in the Delaware Basin by means of brine density flow (abs.): Geological Society of America, Abstracts with Programs, v. 11 , no. 7, p. 379.

Anonymous, 1947, Guadalupe Mountains of New Mexico-Texas: Midland, Texas, West Texas Geological Society Guide Book, Field Trip no. 4, 94 p. 1949, The Permian rocks of Trans-Pecos, Texas region: Midland, Texas, West Texas Geological Society Guide Book, Field Trip no. 4, 94 p.

Babcock, J. A., 1974, The role of algae in the formation of the Capitan Limestone (Permian, Guadalupian), Guadalupe Mountains, West Texas and New Mexico: Madison, University of Wisconsin, unpublished $\mathrm{Ph}$. D. dissertation, 241 p. 
1977, Calcareous algae, organic boundstones, and the genesis of the upper Capitan Limestone (Permian, Guadalupian), Guadalupe Mountains, west Texas and New Mexico, In Hileman, M. E., and Mazzullo, S. J., eds., Jpper Guadalupian facies, Permian reef complex, Guadalupe Mountains, New Mexico and west Texas: Permian Basin Section, Society of Economic Paleontologists and Mineralogists, 1977 Field Conference Guidebook (Publication 77-16), v. 1, p. 3-44.

Babcock, L. C., 1974a, Conodont paleoecology of a Guadalupian (Permian) shelf to basin sequence, Permian reef complex, west Texas and New Mexico (abs.), in Abstracts with Programs: North-central Section, Geological Society of America, p. 489.

$1974 \mathrm{~b}$, Statistical approaches to the conodont paleoecology of the Lamar Limestone, Permian reef complex, west Texas: Madison, University of Wisconsin, unpublished $\mathrm{Ph}$. D. dissertation, $175 \mathrm{p}$.

1977, Life in the Delaware Basin--The paleoecology of the Lamar Limestone, in Hileman, M. E., and Mazzullo, S. J., eds., Upper Guadalupian facies, Permian reef complex, Guadalupe Mountains, New Mexico and west Texas: Permian Basin Section, Society of Economic Paleontologists and Mineralogists, 1977 Field Conference Guidebook (Publication 77-16), v. 1, p. 357-390.

Bachman, G. 0., 1953, Geology of a part of northwestern Mora County, New Mexico: U.S. Geological Survey $0 i 1$ and Gas Investigations Map OM-137. 1975, New Mexico, in McKee, E. D., Crosby E. J., and others, Paleotectonic investigations of the Pennsylvanian System in the United States: U.S. Geological Survey Professional Paper 853-L, p. 233-243.

Baker, C. L., 1920, Contributions to the stratigraphy of eastern New Mexico: American Journal of Science, 4 th ser., v. 49, p. 99-126.

1924, Caverns in the Guadalupe Mountain Range: Science, new ser., v, 59 , p. 379. 1929, Depositional history of the red beds and saline residues of the Texas Permian: Texas University Bulletin 2901, p. 9-72.

1935, Structural geology of trans-Pecos Texas, in The geology of Texas, v. 2: Texas University Bulletin 3401, p. 137-211.

Bal1, S. M., Roberts, J. W., Norton, J. A., and Pollard, W. D., 1971, Queen Formation (Guadalupian, Permian) outcrops of Eddy County, New Mexico, and their bearing on recently proposed depositional models: AAPG Bulletin, v. 55, p. 1348-1355.

Barnes, V. E., ed., 1968, Geologic atlas of Texas, Van Horn-El Paso sheet: Austin, Texas University, Bureau of Economic Geology, 1:250,000 scale map.

Bartlett, J. R., 1854, Personal narrative of explorations and incidents in Texas, New Mexico, California, Sonora and Chihuahua, connected with the United States and Mexican Boundary Commission, during the years 1850, 1851, 1852, and 1853: New York, D. Appleton \& Company, v. 1.

Bates, R. L., 1942, Lateral gradation in the Seven Rivers Formation, Rocky Arroyo, Eddy County, New Mexico: AAPG Bulletin, v. 26, p. 80-99.

Beck, R. H., 1967, Depositional mechanics of the Cherry Canyon Formation, Delaware Basin, Texas: Lubbock, Texas Tech University, unpublished Master's thesis, $107 \mathrm{p}$.

Beede, J. W., 1910, The correlation of the Guadalupian and Kansas sections: American Journal of Science (4th series), v. 30, p. 131-140. 1924, Report on the oil and gas possibilities of the University Block 46 in Culberson County: Texas University Bulletin no. 2346, p. 13-14. 
Behnken, F. H., 1973, Leonardian and Guadalupian (Permian) conodont biostratigraphy and evolution in western and southwestern United States: Madison, University of Wisconsin, unpublished Ph. D. dissertation, $184 \mathrm{p}$.

$1975 \mathrm{a}$, Leonardian and Guadalupian biostratigraphy in western and southwestern United States: Journal of Paleontology, v. 49, p. 284-315. $1975 \mathrm{~b}$, Conodonts as biostratigraphic indices, in Cys, J. M., and Toomey, D. F., eds., Permian exploration, boundaries, and stratigraphy: West Texas Geological Society and Permian Basin Section, Society of Economic Paleontologists and Mineralogists, p. 84-90.

Belt, B. B., and McGlasson, E. H., 1968, Oils from Yeso reservoirs and their basinal equivalents, in Basins of the Southwest, v. 2--American Association of Petroleum Geologists, SW Section, 10th Annual Meeting, Wichita Falls, Texas, 1968: Midland, Texas, West Texas Geological Society, p. 53-67.

Berg, R. R., 1979, Reservoir sandstones of the Delaware Mountain Group, southeast New Mexico, in Sullivan, N. M., ed., Guadalupian Delaware Mountain Group of west Texas and southeast New Mexico: Permian Basin Section, Society of Economic Paleontologists and Mineralogists, 1979 Symposium and Field Conference Guidebook, Publication 79-18, p. 75-95. Bjorklund, L. J., and Motts, W. S., 1959, Geology and groundwater resources of the Carlsbad area, New Mexico: U.S. Geological Survey Open-File Report, $322 \mathrm{p}$.

Black, D. M., 1951a, Origin and development of "positive" water catchment basins, Carlsbad Caverns, New Mexico: National Speleological Society Bulletin 13, p. 27-29. 1951b, Loose carbonate accretions from Carlsbad Caverns, New Mexico: Science, v. 114, p. 126-127. 1952, Cave pearls in Carlsbad Caverns: Science Monthly, v. 74, no. 4, p. 206-210.

1953, Aragonite rafts in Carlsbad Caverns, New Mexico: Science, v. 117, p. 84-85.

Black, T. H., 1954, The origin and development of the Carlsbad Caverns: New Mexico Geological Society Guidebook of southeastern New Mexico, 5th Field Conference, p. 136-142.

Blake, W. P., 1855, Exploration and surveys for a railroad route from the Mississippi River to the Pacific Ocean, V. 2: Washington, D.C.

Blanchard, W. G., Jr., and Davis, M. J., 1929, Permian stratigraphy and structure of parts of southeastern New Mexico and southwestern Texas: AAPG Bulletin, v. 13, p. 957-995.

Bose, Emi1, 1919, The Permo-Carboniferous ammonoids of the Glass Mountains and their stratigraphical significance: Texas University Bulletin 1762, $241 \mathrm{p}$.

Boyd, D. W., 1955, Stratigraphy of the Brokeoff Mountains, New Mexico, in Permian field conference to the Guadalupe Mountains: Permian Basin Section, Society of Economic Paleontologists and Mineralogists, 155 Field Trip Guidebook, p. 47-56. 1958, Permian sedimentary facies, central Guadalupe Mountains, New Mexico: New Mexico Bureau of Mines and Mineral Resources Bulletin 49, New Mexico Institute of Mining and Technology, $100 \mathrm{p}$. 
1962, Leonardian and lower Guadalupian shelf-edge facies in E1 Paso Gap quadrangle, southeastern New Mexico, in Permian of the central Guadalupe Mountains, Eddy County, New Mexico: Hobbs, Roswell and West Texas Geological Societies, Field Trip Guidebook, Publication No. 62-48, p. 9198.

Bozanich, R. G., 1978, The Bell Canyon and Cherry Canyon Formations, southern Delaware Basin: Austin, University of Texas at Austin, unpublished Master's thesis, $165 \mathrm{p}$.

1979, The Bell Canyon and Cherry Canyon Formations, eastern Delaware Basin, Texas: Lithology, environments and mechanisms of deposition, in Sullivan, N. M., ed., Guadalupian Delaware Mountain Group of west Texas and southeast New Mexico: Permian Basin Section, Society of Economic Paleontologists and Mineralogists, 1979 Symposium and Field Conference Guidebook, Publication 79-18, p. 121-141.

Braithwaite, C. J. R., 1973, Reefs: just a problem of semantics?: AAPG Bulletin, v. 57, p. 1100-1116.

Bretz, J. H., 1949, Carlsbad Caverns and other caves of the Guadalupe Block, New Mexico: Journal of Geology, v. 57, p. 447-463.

Brooks, R. P., ed. and compiler, 1964, Ira Rinehart's reference book, Delaware basin exploration, west Texas: Dallas, Texas, Rinehart 0il News Company. , ed. and compiler, 1966, West Texas oil and gas prospects: Dallas, Texas, Rinehart 0il News Company, v. 1, 138 p.

Bullington, N. R., 1968, Geology of the Carlsbad Caverns, in Delaware Basin exploration: West Texas Geological Society, Guidebook, Publication No. 68-55, p. 20-23.

Burke, R. G., 1966a, Sleeping Texas giant stirs again: $0 i 1$ and Gas Journal, v. 64, no 11 , p. 53-55.

1966b, Gas reserves zooming in the Delaware: Oil and Gas Journal, v. 64, no. 24, p. 62-65.

Burnside, R. J., 1959, Geology of part of the Horseshoe atoll in Borden and Howard Counties, Texas: U.S. Geological Survey Professional Paper 315-B, p. 21-35.

Carlson, T. C., and Sipes, L. D. Jr., 1965, Characteristics of San Andres reservoir: Society of Petroleum Engineers of American Institute of Mining Engineers, Permian Basin Section, 5th Oil Recovery Conference, preprints, Paper No. SPE 1145, p. 84-91.

Cartwright, L. D., Jr., 1930, Transverse section of Permian basin, west Texas and southeast New Mexico: AAPG Bulletin, v. 14, p. 969-981.

Cave, H. S., 1954, The Capitan-Castile-Delaware Mountain problem, in New Mexico Geological Society, 5th Field Conference Guidebook, p. 117-124.

Chuber, Stewart, and Rodgers, E. E., 1968, Relationships of oil composition and stratigraphy of Pennsylvanian and Wolfcamp reservoirs, in Basins of the Southwest, v. 2--American Association of Petroleum Geologists, SW Section, 10th Annual Meeting, Wichita Falls, Texas, 1968: Midland, Texas, West Texas Geological Society, p. 29-41.

Clayton, Neal, 1951, Geology and geophysics of the North Snyder area, Scurry County Texas: Geophysics, v. 16, no. 1, p. 1-13.

Clifton, R. L., 1944a, Ammonoids from upper Cherry Canyon Formation of Delaware Mountain group in Texas: AAPG Bulletin, v. 28, p. 1644-1646. 1944b, Paleoecology and environments inferred for some marginal Middle Permian marine strata: AAPG Bulletin, v. 28, p. 1012-1031.

Cooper, G. A., and Grant, R. E., 1966, Permian rock units in the Glass Mountains, west Texas: U.S. Geological Survey Bulletin 1244-E, p. E1-E9. 
Cox, E. R., 1967, Geology and hydrology between Lake McMillan and Carlsbad Springs, Eddy County, New Mexico: U.S. Geological Survey Water Supply Paper $1828,48 \mathrm{p}$.

Craig, D. H., and Schoonmaker, G. R., 1968, Yates oil field, Pecos County, Texas (abs.): AAPG Bulletin, v. 52, no. 3, p. 523.

Crandall, K. H., 1929, Permian stratigraphy of southeastern New Mexico and adjacent parts of western Texas: AAPG Bulletin, v. 13, p. 927-944.

Cromwe11, D. W., 1979, Indian Draw Delaware Field: A model for deeper Delaware sand exploration, in Sullivan, N. M., ed., Guadalupian Delaware Mountain Group of west Texas and southeast New Mexico: Permian Basin Section, Society of Economic Paleontologists and Mineralogists, 1979 Symposium and Field Conference Guidebook, Publication 79-18, p. 142-152.

Cronoble, J. M., 1974, Biotic constituents and origin of facies in Capitan reef, New Mexico and Texas: Mountain Geologist, v. 11, p. 95-108.

Crosby, E. J., and Mape1, W. J., 1975, Central and west Texas, in McKee, E. D., Crosby, E. J., and others, Paleotectonic investigations of the Pennsylvanian System in the United States: U.S. Geological Survey Professional Paper 853-K, p. 197-232.

Cys, J. M., 1971, Origin of Capitan Formation, Guadalupe Mountains, New Mexico and Texas: Discussion: AAPG Bulletin, v. 55, p. 310-312.

1975, New observations on the stratigraphy of key Permian sections of west Texas, in Cys, J. M., and Toomey, D. F., eds., Permian exploration, boundaries, and stratigraphy: West Texas Geological Society and Permian Basin Section, Society of Economic Paleontologists and Mineralogists, p. 22-42.

Cys, J. M., Toomey, D. F., Brezina, J. L., Greenwood, E., Groves, D. B., Klement, K. W., Kullmann, J. D., McMillan, T. L., Schmidt, V., Sneed, E. D., and Wagner, L. H., 1977, Capitan Reef--Evolution of a concept, in Hileman, M. E., and Mazzullo, S. J., eds., Upper Guadalupian facies, Permian reef complex, Guadalupe Mountains, New Mexico and west Texas: Permian Basin Section, Society of Economic Paleontologists and Mineralogists, 1977 Field Conference Guidebook (Publication 77-16), V. I, p. 201-322.

Darton, N. H., and King, P. B., 1932, Western Texas and Carlsbad Caverns: International Geological Congress, 16th, United States 1933, Guidebook 13, Excursion $\mathrm{C}-1,38 \mathrm{p}$.

Darton, N. H., and Reeside, J. B., Jr., 1926, Guadalupe Group: Geological Society of America Bulletin, v. 37, p. 413-428.

David, E. K. (General Chairman), 1977, The oil and gas fields of southeastern New Mexico, 1977 supplement--a symposium: Roswell, New Mexico, Roswell Geological Society, 220 p.

Davies, W. E., and Moore, G. W., 1957, Endellite and hydromagnesite from Carlsbad Caverns: National Speleological Society Bulletin 19, p. 24-27.

Davis, H. E., and others, 1953, North-south cross section through Permian Basin of west Texas: West Texas Geological Society Publication 53-30.

Davis, J. B., and Kirkland, D. W., 1970, Native sulfur deposition in the Castile Formation, Culberson County, Texas: Economic Geology, v. 65, p. 107-121.

Dean, W. E., and Anderson, R. Y., 1974, Trace and minor element variations in the Permian Castile Formation, Delaware basin, Texas and New Mexico, revealed by varve calibration: Fourth International Symposium on Salt, Cleveland, Northern Ohio Geological Society, v. 1, p. 275-285. 
1978, Salinity cycles--evidence for deep-water deposition of the Castile and lower Salado Formations, Delaware basin, Texas and New Mexico, in New Mexico Geological Society, Symposium on Ochoan rocks of southeastern New Mexico and west Texas: New Mexico Bureau of Mines and Mineral Resources Special Publication 159, p. 15-20.

Dean, W. E., Davies, G. R., and Anderson, R. Y., 1975, Sedimentological significance of nodular and laminated anhydrite: Geology, v. 3, p. 367372.

DeFord, R. K., and Lloyd, E. R., 1940, Editorial introduction, Pt. 1 of west Texas-New Mexico symposium: AAPG Bulletin, v. 24, p. 1-14.

DeFord, R. K., and Riggs, G. D., 1941, Tansill Formation, west Texas and southeastern New Mexico: AAPG Bulletin, v. 25, p. 1713-1728.

DeFord, R. K., Riggs, G. D., and Wil1s, N. H., 1938, Surface and subsurface formations, Eddy County, New Mexico (abs.): AAPG Bulletin, v. 22, p. $1706-1707$.

DeFord, R. K., and others, 1951, Apache Mountains of Trans-Pecos Texas: Midland, West Texas Geological Society, 1951 Field Trip Guidebook, 56 p.

Dickey, R. I., 1940, Geologic section from Fisher County through Andrews County, Texas, to Eddy County, New Mexico: AAPG Bulletin, v. 24, p. 3751.

Dodge, C. F., 1958, Delaware Basin: what traps its oil?: Petroleum Engineer, v. 30, p. B48-B52.

Dolton, G. L., Coury, A. B., Frezon, S. E., Robinson, Keith, Varnes, K. L., Wunder, J. M., and Allen, R. W., 1979, Estimates of undiscovered oil and gas, Permian Basin, west Texas and southeast New Mexico: U.S. Geological Survey Open-File Report 79-838, 118 p.

Dunbar, C. 0., 1941, Permian faunas: A study in facies: AAPG Bulletin, v. 52, $\mathrm{p}$. 313-332.

Dunbar, C. O., and Skinner, J. W., 1937, Permian fusulinidae of Texas: Austin, Texas University Bulletin 3701, p. 517-825.

Dunham, R. J., 1965, Vadose pisolite in the Capitan reef (abs.): AAPG Bulletin, v. 49, p. 338.

1969 a, Vadose pisolite in the Capitan reef (Permian), New Mexico and Texas, in Friedman, G. M., ed., Depositional environments in carbonate rocks: Society of Economic Paleontologists and Mineralogists Special Publication 14, p. 182-191.

1969b, Asymmetrically-filled veins in Capitan Reef and their genetic similiarity to vadose pisolite, New Mexico and Texas (abs.): Geological Society of America Special Paper 121, p. 83-84.

1969c, Early vadose silt in Townsend mound (reef) New Mexico, in Freedman, G. M., ed., Depositional environments in carbonate rocks: Society of Economic Paleontologists and Mineralogists Special Publication 14, p. 139-181.

1970, Stratigraphic reefs versus ecologic reefs: AAPG Bulletin, v. 54, p. 1931-1932.

1972, Capitan reef New Mexico and Texas: Facts and questions to aid interpretation and group discussion: Permian Basin Section, Society of Economic Paleontologists and Mineralogists, Publication 72-141, 272 p.

Elam, J. G., 1972, The tectonic style in the Permian Basin and its relationship to cyclicity, in Elam, J. G., and Chuber, S., eds., Cyclic sedimentation in the Permian Basin, 2d ed.: West Texas Geological Society Publication 72-60, p. 55-79.

Esteban, Mateu, 1976, Vadose pisolite and calide: AAPG Bulletin, v. 60, p. 2048-2057. 
Esteban, Mateu, and Pray, L. C., 1975, Subaqueous, syndepositional growth of In-place pisolite, Capitan reef complex (Permian), Guadalupe Mountains, New Mexico and west Texas (abs.): Geological Society of America, Abstracts with Programs, v. 7, p. 1068-1069.

1976, Nonvadose origin of pisolitic facies, Capitan reef complex (Permian), Guadalupe Mountains, New Mexico and west Texas (abs.): AAPG Bulletin, v. 60 , p. 670 .

1977, Origin of the pisolite facies of the shelf crest, in Hileman, M. E., and Mazzullo, S. J., eds., Upper Guadaluplan facies, Permian reef complex, Guadalupe Mountains, New Mexico and west Texas: Permian Basin Section, Society of Economic Paleontologists and Mineralogists, 1977 Field Conference Guidebook (Publication 77-16), v. 1, p. 479-486.

Fiedler, A. G., and Nye, S. S., 1933, Geology and groundwater resources of the Roswell artesian basin, New Mexico: U.S. Geological Survey Water-Supply Paper $639,372 \mathrm{p}$.

Finks, R. M., 1960, Late Paleozoic sponge faunas of the Texas region: American Museum of Natural History Bulletin, v. 120, p. 1-161.

Flawn, P. T., 1956, Basement rocks of Texas and southeast New Mexico: Austin, Texas University, Bureau of Economic Geology, Publication No. 5605, $261 \mathrm{p}$.

Folk, R. L., 1976, Comparative fabrics of length-slow and length-fast aragonite in a Holocene speleothem, Carlsbad Caverns, New Mexico: Journal of Sedimentary Petrology, v. 46, p. 486-496.

Foltz, G. A., 1966, Double X oil field, in The oil and gas fields of southeastern New Mexico: Roswell Geological Society, p. 100-101.

Frenze1, H. N., 1955, The Queen-Grayburg problem, in Permian field conference to the Guadalupe Mountains: Permian Basin Section, Society of Economic Paleontologists and Mineralogists, 1955 Field Trip Guidebook, p. 25-46. 1962, The Queen-Grayburg-San Andres problem solved, in Permian of the central Guadalupe Mountains, Eddy County, New Mexico: West Texas, Roswe11, and Hobbs Geological Society Guidebook, Publication No. 62-48, p. $87-90$.

Friedman, G. M., 1966, Occurrence and origin of Quaternary dolomite of Sa1t Flat, west Texas: Journal of Sedimentary Petrology, v. 36, p. 263-267.

Furnish, W. M., and Glenister, B. F., 1969, The Guadalupian Series (abs.): Geological Society of America Special Paper 121, p. 105-106.

Galley, J. E., 1958, Oil and geology in the Permian Basin of Texas and New Mexico, in Weeks, L. G., ed., Habitat of 0il: New York, American Association of Petroleum Geologists Symposium, p. 395-446.

1971, Summary of petroleum resources in Paleozoic rocks of Region 5-north-central and west Texas and eastern New Mexico, in Cram, I. H., ed., Future petroleum provinces of the United States--their geology and potential: American Association of Petroleum Geologists Memoir 15, V. 1, p. $726-737$.

Gardner, F. J., 1949, West Texas oil: Dallas, Texas, Rinehart 0il News Company, v. 1, 224 p.; v. 2, 275 p.

Gardner, F. J., and Phifer, R. L., 1953, The oil and gas fields of west Texas, Part 1, Railroad Commission District 7-C: Houston, Texas, Five Star 0il Report, $304 \mathrm{p}$.

Gester, G. H., and Hawley, H. J., 1929, Yates Field, Pecos County, Texas, in Structure of typical American oil fields: Tulsa, American Association of Petroleum Geologists, v. 2, p. 480-499. 
Gibson, G. R., 1965, 011 and gas in southwestern region--geologlc framework, in Young, A., and Galley, J. E., eds., Fluids in subsurface environments: American Association of Petroleum Geologists Memoir 4, p. 66-100.

Girty, G. H., 1902, The Upper Permian in western Texas: American Journal of Science, 4th ser., v. 14, p. 363-368.

1908, The Guadalupian fauna: U.S. Geological Survey Professional Paper $58,651 \mathrm{p}$.

1909, The Guadalupian fauna and new stratigraphic evidence: New York Academy of Science Annals, v. 19, p. 137-138.

Good, J. M., 1957, Non-carbonate deposits of Carlsbad Caverns: National Speleological Society Bulletin, v. 19, p. 11-23.

Grant, R. E., 1971, Brachiopods in the Permian reef environment of west Texas: North American Paleontologists Convention Proceedings, Pt. J, p. 1444-1481.

Gratton, P. J. F., and Lemay, W. J., 1969, San Andres oil east of the Pecos, in Summers, W. K., and Kottlowski, F. E. eds., The San Andres limestone, a reservoir for oil and water in New Mexico: New Mexico Geological Society Special Publication 3, p. 37-43.

Grauten, W. F., 1965, Fluid relationships in Delaware Mountain sandstone, in Young, A., and Galley, J. E., eds., Fluids in subsurface environments: American Association of Petroleum Geologists Memoir 4, p. 294-307.

Greenwood, Eugene, 1975, Permian oil and gas production--when, where and why, in Permian exploration, boundaries, and stratigraphy, West Texas

Geological Society and Permian Basin Section, Society of Economic Paleontologists and Mineralogists, Symposium and Field Trip: West Texas Geological Society Publication 75-65, p. 115-126.

Grice, C. R., 1960, The Grice field, Loving County, Texas, in Geology of the Delaware Basin and field trip guidebook: Midland, West Texas Geological Society, 1960 Field Trip Guidebook, p. 78-80.

Guinan, M. A., 1969, Coyanosa Delaware sand, Pecos County, Texas, in Delaware Basin exploration: West Texas Geological Society, Guidebook Publication No. 68-55a, p. 134-137.

1975a, Slide-block geology, Coyanosa and adjacent areas, Pecos and Reeves Counties, Texas (abs.): AAPG Bulletin, v. 55, no. 2, p. 340. $1975 \mathrm{~b}$, More evidence of the slide-block event will follow Delaware basin drilling: 0il and Gas Journal, v. 69, no. 27, p. 120-127.

Haigler, L. B., 1962, Geologic notes on the Delaware Basin: New Mexico Institute of Mining and Technology, Circular 63, $14 \mathrm{p}$.

Haigler, L. B., and Cunningham, R. R., 1972, Structural contour map on top of the undifferentiated Silurian and Devonian rocks in southeastern New Mexico: U.S. Geological Survey $0 i 1$ and Gas Investigations Map OM-218.

Hall, W. E., 1960, Upper Permian correlations in southeastern New Mexico and adjacent parts of west Texas, in Geology of the Delaware Basin and field trip guidebook: West Texas Geological Society, 1960 Field Trip Guidebook, p. 85-88.

Halliday, W. R., 1961, More dolomite speleothems: National Speleological Society Bulletin 19, no. 11, p. 143.

Ham, W. E., 1960, Middle Permian evaporites in southwestern Oklahoma: International Geological Congress, 21st, Copenhagen, Report pt. 12, p. 138-151.

Hardie, C. H., 1958, The Pennsylvanian rocks of the northern Hueco Mountains: West Texas Geological Society Guidebook, Publication No. 5840, p. 43-45. 
Harms, J. C., 1968, Permian deep-water sedimentation by nonturbid currents, Guadalupe Mountains, Texas (abs.): Geological Society of America Special Paper 121, p. 127. 1974, Brushy Canyon Formation, Texas: A deep-water density current deposit: Geological Society of America Bulletin, v. 85, p. 1763-1784.

Harms, J. C., and Pray, L. C., 1974, Erosion and deposition along the MidPermian intracratonic basin margin, Guadalupe Mountains, Texas (abs.): Society of Economic Paleontologists and Mineralogists Special Publication No. 19, p. 37.

Harrington, G. E., 1966, Triste Draw oil field, in the oil and gas fields of southeastern New Mexico: Roswell Geological Society, p. 176-177.

Harrington, J. W., 1963, Opinion of structural mechanics of central basin platform area, west Texas: AAPG Bulletin, v. 47, no. 12, p. 2023-2038.

Harrison, S. C., 1966, Depositional mechanics of Cherry Canyon sandstone tongue: Austin, University of Texas, unpublished Master's thesis, $114 \mathrm{p}$.

Hartman, J. K., and Woodward, L. R., 1971, Future petroleum resources in postMississippian strata of north-central and west Texas, and eastern New Mexico, in Cram I. H., Future petroleum provinces of the United States-their geology and potential: American Association of Petroleum Geologists Memoir 15, v. 2, p. 738-803.

Hayes, P. T., 1957, Geology of the Carlsbad Caverns East quadrangle, New Mexico: U.S. Geological Survey Quadrangle Map GQ 98.

1959, San Andres Limestone and related Permian rocks in Last Chance Canyon and vicinity, southeastern New Mexico: AAPG Bulletin, v. 43, p. 2197-2213.

1964, Geology of the Guadalupe Mountains, New Mexico: U.S. Geological

Survey Professional Paper 446, 69 p.

Hayes, P. T., and Koogle, R. L., 1958, Geology of the Carlsbad Caverns West quadrangle, New Mexico-Texas: U.S. Geological Survey Quadrangle Map GQ 112 .

Hecke1, P. H., 1974, Carbonate buildups in the geologic record: a review, in Laporte, L. F., ed., Reefs in time and space: Society of Economic Paleontologists and Mineralogists Special Publication 18, p. 90-154.

Hennen, R. V., and Metcalf, R. J., 1929, Yates oil pool, Pecos County, Texas: AAPG Bulletin, V. 13, no. 12, p. 1509-1556.

Herald, F. A., ed., 1957, Occurrence of oil and gas in west Texas: Austin, Texas University Publication 5716, $442 \mathrm{p}$.

Hess, F. L., 1929, Oolites or cave pearls in the Carlsbad Caverns: U.S. National Museum Proceedings, v. 76, art. 16, 5 p.

Hills, J. M., 1942, Rhythm of Permian seas--a paleogeographic study: AAPG Bulletin, v. 26, p. 217-255.

1968, Gas in Delaware and Val Verde Basins, west Texas and southeast New Mexico, in Beebe, B. W., and Curtis, B. F., eds., Natural gases of North American, a symposium: American Association of Petroleum Geologists Memoir 9, v. 2, p. 1394-1492. 1970, Late Paleozoic structural directions in southern Permian Basin, west Texas and southeastern New Mexico: AAPG Bulletin, v. 54, no. 10, p. 1809-1827.

1972, Late Paleozoic sedimentation in west Texas Permian Basin: AAPG Bulletin, v. 56, p. 2303-2322. 
1979, Delaware Basin sedimentation, tectonism and hydrocarbon generation, in Sullivan, N. M., ed., Guadalupian Delaware Mountain Group of west Texas and southeast New Mexico: Permian Basin Section, Society of Economic Paleontologists and Mineralogists, 1979 Symposium and Field Conference Guidebook, Publication 79-18, p. 1 .

Hinds, J. S., and Cunningham, R. R., 1970, Elemental sulfur in Eddy County, New Mexico: J.S. Geological Survey Circular 628, 13 p.

Hiss, W. L., 1977a, Movement of ground water in Permian Guadalupian aquifer systems, southeastern New Mexico and west Texas (abs.), in Hileman, M. E., and Mazzullo, S. J., eds., Upper Guadalupian facies, Permian reef complex, Guadalupe Mountains, New Mexico and west Texas: Permian Basin Section, Society of Economic Paleontologists and Mineralogists, 1977 Field Conference Guidebook (Publication 77-16), v. 1, p. 487. $1977 b$, Fresh-saline water interface in Permian Guadalupian Capitanaquifer, southwest of Carlsbad, Eddy County, New Mexico (abs.), in Hileman, M. E., and Mazzullo, S. J., eds., Upper Guadalupian facies, Permian reef complex, Guadalupe Mountains, New Mexico and west Texas: Permian Basin Section, Society of Economic Paleontologists and Mineralogists, 1977 Field Conference Guidebook (Publication 77-16), v. 1, p. 488.

Hobbs, Roswe11, and West Texas Geological Societies 1962 Field Trip Committee, 1962, Permian of the central Guadalupe Mountains, Eddy County, New Mexico: Hobbs, Roswell and West Texas Geological Societies Field Trip Guidebook and Geological Discussions, Publication No. 62-48, 115 p.

Hollingsworth, R. V., and Williams, H. L., 1955, Evolution of the Fusulinidae: Midland, Texas, Paleontological Laboratory, 19 p.

Holmquest, H. H., 1965, Deep pays in Delaware and Val Verde basins, in Young, A., and Galley, J. E., eds., Fluids in subsurface environments: American Association of Petroleum Geologists Memoir 4, p. 257-279.

Hopkins, Eldon, 1974, Permian Basin has fifth of U.S. well completions: The Drilling Contract, v. 30, no. 5, p. 60-61.

Horak, R. L., 1975, Tectonic relationship of the Permian Basin to the Basin and Range Province, in Hills, J. S., ed., Exploration from the mountains to the basin, American Association of Petroleum Geologists, SW Section, and Society of Economic Paleontologists and Mineralogists, Permian Basin section 1975, Joint Meeting, Transactions: E1 Paso Geological Society, p. $1-94$.

Horberg, L., 1949, Geomorphic history of the Carlsbad Caverns area, New Mexico: Journal of Geology, v. 57, p. 464-476.

Horst, G. F., and Wilson, D. A., 1969, Log evaluation and wireline operations in the Delaware Basin, in Delaware Basin exploration: West Texas Geological Society, Guidebook, Publication No. 68-55a, p. 111-117.

Hul1, J. P. D., Jr., 1957, Petrogenesis of Permian Delaware Mountain sandstone, Texas and New Mexico: AAPG Bulletin, v. 41, p. 278-307.

Jacka, A. D., 1974, Replacement of fossils by length-slow chalcedony and associated dolomitization: Journal of Sedimentary Petrology, v. 44, p. $421-427$.

1979, Deposition and entrapment of hydrocarbons in Bell Canyon and Cherry Canyon deep-sea fans of the Delaware Basin, in Sullivan, N. M., ed., Guadalupian Delaware Mountain Group of west Texas and southeast New Mexico: Permian Basin Section, Society of Economic Paleontologists and Mineralogists, 1979 Symposium and Field Conference Guidebook, Publication $79-18$, p. $104-120$. 
Jacka, A. D., Beck, R. H., St. Germain, L. C., and Harrison, S. C., 1968, Permian deep-sea fans of the Delaware Mountain Group (Guadalupian), Delaware basin, in Guadalupian facies, Apache Mountains area, west Texas: Permian Basin Section, Society of Economic Paleontologists and Mineralogists, p. 49-90.

Jacka, A. D., and Franco, L. A., 1974, Deposition and diagenes is of Permian evaporites and associated carbonates and clastics on shelf areas of the Permian Basin: Fourth Symposium on Salt, Northern Ohio Geological Soclety, v. I, p. 67-89.

Jacka, A. D., Thomas, C. M., Beck, R. H., Williams, K. W., and Harrison, S. C., 1972, Guadalupian depositional cycles, Delaware Basin and Northwest Shelf, in Elam, J. G., and Chuber, S., eds., Cyclic sedimentation in the Permian Basin (second edition): West Texas Geological Society Publication 72-60, p. 151-195.

Jagnow, D. H., 1979, Cavern development in the Guadalupe Mountains: Columbus, Ohio, Cave Research Foundation, 55 p.

Jenkins, R. E., 1961, Characteristics of the Delaware Formation: Journal of Petroleum Technology, v. 13, p. 1230-1236.

Jenney, W. P., 1874, Notes on the geology of western Texas near the thirtysecond parallel: American Journal of Science, 3rd ser., v. 7, p. 25-28.

Johnson, J. H., 1942, Permian lime-secreting algae from the Guadalupe Mountains, New Mexico: Geological Society of America Bulletin, v. 53, p. 195-226.

1951, Permian calcareous algae from the Apache Mountains, Texas:

Journal of Paleontology, v. 25, p. 21-30.

1963, Pennsylvanian and Permian algae: Golden, Colorado School of Mines Quarterly, v. 58, no. 3, 211 p.

Jones, C. L., 1954, The occurence and distribution of potassium minerals in southeastern new Mexico, in Guidebook of southeastern New Mexico: New Mexico Geological Society, Guidebook, 5th Field Conference, p. 107-112.

Jones, C. L., and Madsen, B. M., 1968, Evaporite geology of Fifth ore zone, Carlsbad district, southeastern New Mexico: U.S. Geological Survey Bulletin 1252-B, p. B1-B21.

Jones, T. S., and Smith, H. M., 1965, Relationship of oil composition and stratigraphy in Permian Basin of west Texas and New Mexico, in Young, A., and Galley, J. E., eds., Fluids in subsurface environments, a symposium: American Association of Petroleum Geologists Memoir 4, p. 101-224.

Jones, T. S., and others, 1949, East-west cross section through Permian Basin of west Texas: West Texas Geological Society Publication 49-17.

Keller, D. T., and Porter, W. C., 1972, Developments in west Texas and eastern New Mexico: AAPG Bulletin, v. 56, no. 7, p. 1264-1268.

Kelley, V. C., 1971, Geology of the Pecos county, southeastern New Mexico: New Mexico Bureau of Mines and Mineral Resources, Memoir 24, 75 p. 1972, Geometry and correlation along Permian Capitan escarpment, New Mexico and Texas: AAPG Bulletin, v. 56, p. 2192-2211.

Kenda11, C. G. St. C., 1969, An environmental re-interpretation of the Permian evaporite-carbonate shelf sediments of the Guadalupe Mountains: Geological Society of America Bulletin, v. 80, p. 2503-2526.

Kerr, S. D., and Thompson, A., 1963, Origin of nodular and bedded anhydrite in Permian shelf sediments, Texas and New Mexico: AAPG Bulletin, v. 47, p. 1726-1732. 
1967, Reef and associated deposits in the Permian of west Texas, in McKee, E. D., and others, Paleotectonic maps of the Permian System: U.S. Geological Survey Miscellaneous Geological Investigations Map I-450 (with text), p. 36-44.

Keyes, C. R., 1929, Guadalupian reef theory: Pan American Geologist, v. 52, p. 41-60.

1933, Capitan Limestone as great barrier reef (abs.): Pan American Geologist, v. 60 , p. 306.

1936, Guadalupian Series: its span and affinites: Pan American Geologist, v. 65, p. 35-36.

1938a, Guadalupian fauna; what it is not: Pan American Geologist, v. 69 , p. 139-144.

1938b, Guadalupian Series in taxonomic status: Pan American Geologist, v. 69, p. 237-240.

King, P. B., 1926, The geologic structure of a portion of the Glass Mountains of west Texas: AAPG Bulletin, v. 10, p. 877-884.

1930, The geology of the Glass Mountains: Austin, Texas University

Bulletin 3038, pt. 1 , 167 p.

1934, Permian stratigraphy of Trans-Pecos Texas: Geological Society of America Bulletin, v. 45, p. 697-798.

1935, Outline of structural development of Trans-Pecos Texas: AAPG

Bulletin, v. 19, p. 221-261.

1936a, Unconformities in the later Paleozoic of Trans-Pecos Texas:

Texas University Bulletin 3501, p. 131-135.

1936b, Permian rocks of the southern Guadalupe Mountains: Tulsa

Geological Society Digest for 1936, p. 37-42.

1942, Permian of west Texas and southeastern New Mexico: AAPG Bulletin, v. 26, p. 535-763.

1947, Permian correlations: AAPG Bulletin, v. 31, p. 774-777.

1948, Geology of the southern Guadalupe Mountains, Texas: U.S.

Geological Survey Professional Paper 215, 183 p.

1949, Regional geologic map of parts of Culberson and Hudspeth Counties, Texas: U.S. Geological Survey $0 i 1$ and Gas Investigations, Preliminary Map 90.

1965, Geology of the Sierra Diablo region, west Texas: U.S. Geological

Survey Professional Paper 480, 185 p.

1967, Reef and associated deposits in the Permian of west Texas in

McKee, E. D., and others, Paleotectonic maps of the Permian System: U.S. Geological Survey, Miscellaneous Investigations Map I-450 (with text), p. 36-44.

King, P. B., and Fountain, H. C., 1944, Geologic map of southern Guadalupe Mountains, Hudspeth and Culberson Counties, Texas: U.S. Geological Survey $0 i 1$ and Gas Investigations, Preliminary Map 18.

King, P. B., and King. R. E., 1928, The Pennsylvanian and Permian stratigraphy of the Glass Mountains: Texas University Bulletin 2801, p. 109-145.

1929, Stratigraphy of outcropping Carboniferous and Permian rocks of Trans-Pecos Texas: AAPG Bulletin, v. 13, p. 907-926.

King, P. B., and Knight, J. B., 1944, Sierra Diablo Region, Hudspeth and Culberson Counties, Texas: U.S. Geological Survey, 011 and Gas Investigations Preliminary Map 2.

1945, Geology of Hueco Mountains, E1 Paso and Hudspeth Counties, Texas: U.S. Geological Survey, 011 and Gas Investigations Preliminary Map no. 36, 2 sheets. 
King, P. B., and Newe11, N. D., 1956, McCombs Limestone member of Bel1 Canyon Formation, Guadalupe Mountains, Texas: AAPG Bulletin, v. 40, p. 386-387.

King, R. E., 1931, The geology of the Glass Mountains: Austin, Texas University Bulletin 3042, pt. 2, 245 p.

King, R. E., Bates, R. L., Hills, J. M., Martin, B. G., and Taylor, S. J., 1942, Resume of geology of the south Permian Basin, Texas and New Mexico: Geological Society of America Bulletin, v. 53, p. 539-560.

King, R. H., 1947, Sedimentation in Permian Castile sea: AAPG Bulletin, V. 31, p. 470-477.

Kinney, E. E., and Schatz, F. L. (chairmen), 1967, The oil and gas fields of southeastern New Mexico, 1966 supplement, a symposium: Roswell, New Mexico, Roswell Geological Society, 185 p.

Kirkland, D. W., and Anderson, R. Y., 1970, Microfolding in the Castile and Todilto evaporites, Texas and New Mexico: Geological Society of America Bulletin, v. 81, p. 3259-3282.

Kirkland, D. W., and Evans, Robert, 1976, Origin of limestone buttes, gypsum plain, Culberson County, Texas: AAPG Bulletin, v. 60, p. 2005-2018.

Klement, K. W., 1966, Studies on the ecological distribution of lime-secreting and sediment-trapping algae in reefs and associated environments: Neues Jahrbuch fur Geologie und Palaontologie, Abhandlungen, v. 125, p. 363381.

Koss, G. M., 1977, Carbonate mass flow sequences of the Permian Delaware Basin, west Texas, in Hileman, M. E. and Mazzullo, S. J., eds., Upper Guadalupian facies, Permian reef complex, Guadalupe Mountains, New Mexico and west Texas: Permian Basin Section, Society of Economic Paleontologists and Mineralogists, 1977 Field Conference Guidebook (Publication 77-16), v. 1, p. 391-408.

Kroenlein, G. A., 1939, Salt, potash, and anhydrite in Castile Formation of southeast New Mexico: AAPG Bulletin, v. 23, p. 1682-1693.

Lang, W. B., 1935, Upper Permian formations of Delaware Basin of Texas and New Mexico: AAPG Bulletin, v. 19, p. 962-970. 1937, The Permian formations of the Pecos Valley of New Mexico and Texas: AAPG Bulletin, v. 21, p. 833-898. 1939, Salado Formation of the Permian Basin: AAPG Bulletin, v. 23, p. 1569-1572.

1941, New source of sodium sulphate: AAPG Bulletin, v. 25, p. 152-160. 1942, Basal beds of Salado Formation in Fletcher potash core test, near Carlsbad, New Mexico: AAPG Bulletin, v. 26, p. 63-79.

LeMay, W. J., 1960, Abo reefing in southeastern new Mexico, in Sweeney, H. N., ed., The oil and gas fields of southeastern New Mexico, 1960 supplement, a symposium: Roswe11, New Mexico, Roswell Geological Society, p. XVIIIXXI.

Lewis, F. E., 1941, Position of San Andres group, west Texas and New Mexico: AAPG Bulletin, v. 25, p. 73-103.

Lloyd, E. R., 1929, Capitan limestone and associated formations of New Mexico and Texas: AAPG Bulletin, v. 13, p. 645-658.

1931, (published posthumously in 1975), Barrier reefs and saline residues of the Permian Basin, in Cys, J. M., and Toomey, D. F., eds., Permian exploration, boundaries, and stratigraphy: West Texas Geological Society and Permian Basin Section, Society of Economic Paleontologists and Mineralogists, p. 1-21.

1949, Pre-San Andres stratigraphy and oil-producing zones in southeastern New Mexico: New Mexico Bureau of Mines and Mineral Resources, Bulletin 29, 87 p. 
1952, Correlation chart, Permian of west Texas and New Mexico: Midland, Texas, West Texas Geological Society.

1953, Reefs and associated rocks: Dil and Gas Journal, v. 52, p. 268270.

Long, W. T. B., 1942, The Carlsbad dolomite and the pisolites of the Guadalupe Mountains of New Mexico, (abs.): AAPG Bulletin, v. 26, no. 5, p. 901.

Loucks, R. G., and Folk, R. L., 1976, Fanlike rays of former aragonite in Permian Capitan reef pisolite: Journal of Sedimentary Petrology, v. 46, p. 483-485.

Lucia, F. J., 1961, Dedolomitization in the Tansill (Permian) Formation: Geological Society of America Bulletin, v. 72, p. 1107-1109.

Maher, J. C., ed., 1960, Stratigraphic cross section of Paleozoic rocks--west Texas to northern Montana: Tulsa, Oklahoma, American Association of Petroleum Geologists.

Maley, V. C., and Huffington, R. M., 1953, Cenozoic fill and evaporite solution in the Delaware Basin, Texas and New Mexico: Geological Society of America Bulletin, v. 64, p. 539-546.

Marshall, J. W., 1952, Spraberry reservoir of west Texas: AAPG Bulletin, v. 36 , no. 11, p. 2189-2191.

Marshal1, W. S., 1954, Varve-like laminations in the Permian Bone Spring Limestone of western Texas: New York, Columbia University, unpublished Master's thesis, $48 \mathrm{p}$.

Mazzul1o, S. J., 1977, Synsedimentary diagenesis of reefs, in Hileman, M. E., and Mazzullo, S. J., eds., Upper Guadalupian facies, Permian reef complex, New Mexico and west Texas: Permian Basin Section, Society of Economic Paleontologists and Mineralogists, 1977, Field Conference Guidebook (Publication 77-16), v. 1, p. 323-356.

Mazzullo, S. J. and Cys, J. M., 1977, Submarine cements in Permian boundstones and reef-associated rocks, Guadalupe Mountains, west Texas and southeastern New Mexico, in Hileman, M. E., and Mazzullo, S. J., eds., Upper Guadalupian facies, Permian reef complex, Guadalupe Mountains, New Mexico and west Texas: Permian Basin Section, Society of Economic Paleontologists and Mineralogists, 1977 Field Conference Guidebook (Publication 77-16), v. 1, p. 151-200.

McDaniel, P. N., and Pray, L. C., 1967, Bank to basin transition in Permian (Leonardian) carbonates, Guadalupe Mountalns (abs.): AAPG Bulletin, V. 51, p. 474 .

McKee, E. D., 1951, Sedimentary basins of Arizona and adjoining areas: Geological Society of America Bulletin, v. 62, p. 481-506.

McKee, E. D., Oriel, S. S., and others, 1967, Paleotectonic maps of the Permian System: U.S. Geological Survey, Miscellaneous Geological Investigations Map I-450, 164 p.

McLennan, Lamar, Jr., and Bradley, H. W., 1951, Spraberry and Dean sandstones of west Texas: AAPG Bulletin, v. 35, no 4, p. 899-908.

McNeal, R. P., 1965, Hydrodynamics of the Permian basin, in Young, A., and Galley, J. E., eds., Fluids in subsurface environments: American Association of Petroleum Geologists Memoir 4, p. 308-326.

McNeal, R. P., and Mooney, T. D., 1968, Relationships of oil composition and stratigraphy of Delaware reservoirs, in Basins of the Southwest: $v .2$, p. 68-75. Also reprinted in Sullivan $\bar{N}$. M., ed., Guadalupian Delaware Mountaln Group of west Texas and southeast New Mexico: Permian Basin Section, Society of Economic Paleontologists and Mineralogists, 1979 Symposium and Field Conference Guidebook, Publication 79-18, p. 183-190. 
Mear, C. E., and Yarbrough, D. V., 1961, Yates Formation in southern Permian Basin of west Texas: AAPG Bulletin, v. 45, p. 1545-1556.

Meinzer, O. E., Renick, B. C., and Bryan, K., 1926, Geology of No. 3 Reservoir Site of the Carlsbad Irrigations Project, New Mexico, with reference to water-tightness: U.S. Geological Survey Water-Supply Paper 580-A, 39 p.

Meissner, F. F., 1972, Cyclic sedimentation in Middle Permian strata of the Permian basin, in Elam, J. G., and Chuber, S., eds., Cyclic sedimentation in the Permian Basin, second edition: West Texas Geological Society Publication 72-60, p. 203-232.

Midland Map Company, 1978, Producing zone map, the Permian Basin, west Texas and southeast New Mexico, Midland, Texas, Midland Map Co., 1 sheet.

Miller, A. K., and Furnish, W. M., 1940, Permian ammonoids of the Guadalupe Mountain Region and adjacent areas: Geological Society of America Special Paper 26.

Miller, S. T., 1969, Summary of geophysical exploration in the Delaware Basin, in Delaware Basin exploration: West Texas Geological Society, Guidebook, Publication No. 68-55a, p. 105, 110.

Moore, G. W., 1959, Alteration of gypsum to form the Capitan Limestone of New Mexico and Texas (abs.): Geological Society of America Bulletin, v. 70, p. 1647.

1960, Geology of Carlsbad Caverns, New Mexico, in Spangle, P. F., ed., A guidebook to Carlsbad Caverns National Park: Washington, D.C., The National Speleological Society, Guidebook Series No. 1, p. 10-17.

Moran, W. R., 1954, Proposed type section for the Queen and Grayburg Formations of Guadalupian age in the Guadalupe Mountains, Eddy County, New Mexico (abs.): Geological Society of America Bulletin, v. 65, p. 1288.

1955, Sandstone in New Mexico Room of Carlsbad Caverns, New Mexico: AAPG Bulletin, v. 39, p. 256-259. 1962, Surface type localities of the Queen and Grayburg Formations in the Guadalupe Mountains, Eddy County, New Mexico, in Permian of the central Guadalupe Mountains, Eddy County, New Mexico: Hobbs, Roswell, and West Texas Geological Societies Guidebook, Publication 62-48, p. 7686.

Motts, W. S., 1959, Age of the Carlsbad Caverns and related caves in the rocks of Guadalupe age west of the Pecos River in southeastern New Mexico

(abs.): Geological Society of America Bulletin, v. 70, no. 12, pt. 2, p. 1737.

1962a, Generalized geology of part of the Guadalupe Mountains and vicinity, in Permian of the Central Guadalupe Mountains, Eddy County, New Mexico: Hobbs, Roswell, and West Texas Geological Societies Guidebook, Publication 62-48, p. 99-100.

1962b, Geology of the West Carlsbad quadrangle, New Mexico: U.S.

Geological Survey Geologic Quadrangle Map GQ-167.

1968, The control of ground-water occurrence by lithofacies in the

Guadalupian reef complex near Carlsbad, New Mexico: Geological Society of America Bulletin, v. 79, p. 283-298.

1972, Geology and paleoenvironments of the northern segment, Capitan shelf, New Mexico and west Texas: Geological Society of America Bulletin, v. 83, p. 701-722.

1973, Structure, sedimentation and paleoenvironments of northern Capitan reef complex, New Mexico and west Texas (abs.): AAPG Bulletin, v. 57, p. 796. 
Mutch, T. A., 1966, Abundance of magnetic spherules in Silurian and Permian salt samples: Earth and Planetary Science Letters, v. 1, p. 325-329.

Myers, S. D., 1973, The Permian Basin--Petroleum empire of the southwest: E1 Paso, Texas, Permian Press, 708 p.

1977, The Permian Basin--Petroleum empire of the southwest, era of advancement: E1 Paso, Texas, Permian Press, $624 \mathrm{p}$.

Narin, A. E. M., and Smithwick, M. E., 1976, Permian paleogeography and climatology, in Falke, H., ed., The continental Permian in central, west, and south Europe: Dordrecht, Holland, D. Reidel Publishing Co., p. 282312.

Needham, C. E., 1937, Some New Mexico Fusulinidae: New Mexico School of Mines Bulletin 14, $88 \mathrm{p}$.

Needham, C. E., and Bates, R. L., 1943, Permian type sections in central New Mexico: Geological Society of America Bulletin, v. 54, p. 1653-1667.

Neese, D. A. and Schwartz, A. H., 1977, Facies mosaic of the upper Yates and lower Tansill Formations, Walnut and Rattlesnake Canyons, Guadalupe Mountains, New Mexico, in Hileman, M. E, and Mazzullo, S. J., eds., Jpper Guadalupian facies, Permian reef complex, Guadalupe Mountains, New Mexico and west Texas: Permian Basin Section, Society of Economic Paleontologists and Mineralogists, 1977 Field Conference Guidebook (Publication 77-16), v. 1, p. 437-450.

Nelson, L. A., and Haigh, B. R., 1958, Franklin and Hueco Mountains, Texas: West Texas Geological Society Guidebook, 1958 Field Trip, 91 p.

New Mexico Geological Society, 1954, Guidebook of southeastern New Mexico: 5th Field Conference, 1954, 209 p.

Newe11, N. D., 1955, Depositional fabric in Permian reef 1imestone: Journal of Geology, v. 63, p. 301-309.

Newel1, N. D., Rigby, J. K., Driggs, A., Boyd, D. W., and Stehli, F. G., 1976, Permian reef complex, Tunisia: Brigham Young University Studies, v. 23, pt. 1, p. 75-112.

Newell, N. D., Rigby, J. K., Fisher, A. G., Whiteman, A. J., Hickox, J. E., and Bradley, J. S., 1953, The Permian reef complex of the Guadalupe Mountains region, Texas and New Mexico: San Francisco, Freeman and Company, $236 \mathrm{p}$.

Nottingham, M. W., 1960, Recent Bell Canyon exploration in the north Delaware Basin (New Mexico-Texas), in Natural gases in the Southwest:

Southwestern Federation of Geological Socleties Transactions, v. 1, p. 139-153.

Olive, W. W., 1957, Solution-subsidence troughs, Cast1le formation of Gypsum Plain, Texas and New Mexico: Geological Society of America, Bulletin, v. 68 , p. 351-358.

Oriel, S. S., Myers, D. A., and Crosby E. J., 1967, West Texas Permian Basin region, In Paleotectonic Investigations of the Permian System in the United States: J.S. Geological Survey Professional Paper 515-C, p. C17-C60.

Otte, Care1, Jr., and Parks, J. M., Jr., 1963, Fabric studies of V1rg 1 and Wolfcamp bioherms, New Mexico: Journal of Geology, v. 71, p. 380-396.

Page, L. R., and Adams, J. E., 1940, Eastern Midland Basin, Texas: AAPG Bulletin, v. 24, p. 52-64.

Payne, M. W., 1973, Basinal sandstone facies of the Delaware Mountaln Group, west Texas and southeast New Mexico: College Station, Texas $A$ and $M$ University, unpublished $\mathrm{Ph}$. D, dissertation, $150 \mathrm{p}$.

1976, Basinal sandstone facies, Delaware basin, west Texas and southeast New Mexico: AAPG Bulletin, v. 60, p. 517-527. 
1979, Submarine-fan channel depositional processes in the Permian Bell Canyon Formation, west Texas and southeast New Mexico, in Sullivan, N. M. ed., Guadaluplan Delaware Mountain Group of west Texas and southeast New Mexico: Permian Basin Section, Society of Economic Paleontologists and Mineralogists, 1979 Symposium and Field Conference Guidebook, Publication 79-18, p. 96-103.

Pia, J. V., 1940, Vorlaufige Ubersicht der Kalkalgen des Perms von Nordamerika: Akademie der Wissenschaften, Wien, Math.-Naturwiss. K1., Anz. 9, preprint, June 13, p. 1-9.

Plummer, F. B., and Scott, Gayle, 1937, Upper Paleozolc ammonites in Texas, in The geology of Texas, v. 3: Texas University Bulletin 3701, p. 13-156.

Porch, E. L., Jr., 1917, The Rustler Springs sulfur deposits: Texas University Bulletin, no. $1722,71 \mathrm{p}$.

Pratt, W. E., 1954, Evidences of Igneous activity in the northwestern part of the Delaware basin, in Guidebook of southeastern New Mexico: New Mexico Geological Society, Guidebook, 5th Field Conference, p. 143-147.

Pray, L. C., 1971, Submarine slope erosion along Permian bank margin, west Texas (abs.): AAPG Bulletin, v. 55, p. 358 .

1975, Basin facies carbonates and associated features of the Guadalupe Mountain escarpment, Texas: Preliminary guidebook for Field Trip No. 2, Society of Economic Paleontologists and Mineralogists Annual Convention, (Dallas, Texas), $16 \mathrm{p}$.

1977, The all wet constant sea level hypothesis of Upper Guadalupian shelf and shelf edge strata, Guadalupe Mountains, New Mexico and Texas, in Hileman, M. E. and Mazzullo, S. J., eds., Upper Guadalupian facies, Permian reef complex, Guadalupe Mountains, New Mexico and west Texas: Permian Basin Section, Society of Economic Paleontologists and Mineralogists, 1977 Field Conference Guidebook (Publication 77-16), v. 1, p. 433-436.

Pray, L. C., and Esteban, Mateu, eds., 1977, Upper Guadalupian facies, Permian reef complex, Guadalupe Mountains, New Mexico and west Texas; Volume 2, Road logs and locality guides: Permian Basin Section, Society of Economic Paleontologists and Mineralogists, 1977 Field Conference Guidebook, Publication 77-16, 194 p.

Pray, L. C., and Stehli, F. G., 1962, Allochthonous origin, Bone Spring "patch reefs", west Texas (abs.): Geological Society of American Special Paper 73 , p. 118A-119A.

Richardson, G. B., 1904, Report of reconaissance in Trans-Pecos Texas north of the Texas and Pacific Railway: Texas University Bulletin no. 23, 119 p. 1914, Van Horn folio, Texas: U.S. Geological Survey Folio 194.

Rigby, J. K., 1952, Paleoecology of the Delaware Mountain group, Guadalupe Mountains area, Texas and New Mexico: New York, Columbia University, unpublished $\mathrm{Ph}$. D., Thesis, 286 p.

1953, Some transverse stylolites, Guadalupe Mountains: Journal of Sedimentary Petrology, v. 23, no. 4, p. 265-271. 1957, Relationships between Acanthocladia guadalupensis and Solenopora texana and the bryozoan-algal consortium hypothesis: Journal of Paleontology, v. 31, p. 603-606. 1958, Mass movement in Permian rocks of Trans-Pecos Texas: Journal of Sedimentary Petrology, v. 28, p. 298-315.

Roswell Geological Society, 1951, Permian stratigraphy of the Capitan Reef area of the southern Guadalupe Mountains, New Mexico: Roswell Geologica1 Society, 1951 Field Conference Guidebook, 20 p. 
1952, Surface structures of the foothill region of the Sacramento and Guadalupe Mountains: Roswell Geological Society, 1952 Field Conference Guidebook, 14 p.

1957, Slaughter Canyon, New Cave and Capitan Reef exposures, Carlsbad Caverns National Park: Roswell Geological Society (April 13, 1957) Field Trip Guidebook, 19 p.

1964, Geology of the Capitan reef complex of the Guadalupe Mountains, Culberson County, Texas and Eddy County, New Mexico: Roswell Geological Society, 1964 Field Trip Guidebook, 124 p.

Roth, R. I., 1942, West Texas barred basin: Geological Society of America Bulletin, v. 53, p. 1659-1674.

Ruedemann, Rudolf, 1929, Coralline algae, Guadalupe Mountains: AAPG Bulletin, v. 13, p. 1079-1080.

St. Germain, L. C., 1966, Depositional dynamics of the Brushy Canyon Formation, Delaware basin: Lubbock, Texas Tech University, unpublished Master's thesis, $119 \mathrm{p}$.

Sarg, J. F., 1976, Sedimentology of the carbonate-evaporite facies transition of the Seven Rivers Formation (Guadalupian, Permian) in southeast New Mexico: Madison, University of Wisconsin, unpublished $\mathrm{Ph} . \mathrm{D}$. dissertation, $313 \mathrm{p}$.

1977, Sedimentology of the carbonate-evaporite facies transition of the Seven Rivers Formation (Guadalupian, Permian) in southeast New Mexico, in Hileman, M. E., and Mazzullo, S. J., eds., Jpper Guadalupian facies, Permian reef complex, Guadalupe Mountains, New Mexico and west Texas: Permian Basin Section, Society of Economic Paleontologists and Mineralogists, 1977 Field Conference, Guidebook (Publication 77-16), v. 1, p. 451-478.

Sax, N. A., and Stenzel, W. K., 1968, Oils from Abo reservoirs of the northwest shelf, in Basins of the southwest, Volume 2: American Association of Petroleum Geologists, Southwest Section, 10th Annual Meeting, Wichita Falls, Texas, 1968: Midland, Texas, West Texas Geological Society, p. 42-52.

Scalapino, R. A., 1950, Development of ground water for irrigation in the Dell City Area, Hudspeth County, Texas: Texas Board of Water Engineers Bulletin 5004.

Schmidt, Volkmar, 1977, Inorganic and organic reef growth and subsequent diagenesis in the Permian Capitan reef complex, Guadalupe Mountains, Texas, New Mexico, in Hileman, M. E., and Mazzullo, S. J., eds., Jpper Guadalupian facies, Permian reef complex, Guadalupe Mountains, New Mexico and west Texas: Permian Basin Section, Society of Economic Paleontologists and Mineralogists, 1977 Field Conference Guidebook (Publication 77-16), v. 1, p. 93-132.

Schmidt, Volkmar, and K. W. Klement, 1971, Early diagenetic origin of reef framework in the Permian reef complex, Guadalupe Mountains, Texas and New Mexico (abs.): International Sedimentology Congress, Program with Abstracts, p. 89.

Schmitt, G. T., 1954, Genesis and depositional history of Spraberry formation, Midland basin, Texas: AAPG Bulletin, v. 38, no. 9, p. 1957-1978.

Scholle, P. A., and Kinsman, D. J. J., 1974, Aragonitic and high-Mg calcite caliche from the Persian Gulf--a modern analog for the Permian of Texas and New Mexico: Journal of Sedimentary Petrology, v. 44, p. 904-916.

Schultz, C. B., and Howard, E. B., 1935, The fauna of Burnet Cave, Guadalupe Mountains, New Mexico: Academy of Natural Sciences Proceedings, v. 87, p. 273-298. 
Scobey, W. B., and others, 1951, North-south cross section through Permian Basin of west Texas: West Texas Geological Society, Publication 51-27.

Scott, R. J., 1966, Paduca oil field, in The oil and gas fields of southeastern New Mexico: Roswe11 Geological Society, p. 144-145.

Seewa1d, K. 0., 1969, Pennsylvanian and Lower Permian stratigraphy, Hueco Mountains, Texas, in Delaware Basin exploration: West Texas Geological Society, Guidebook, Publication No. 68-55a, p. 45-49.

Shaller, W. T., and Henderson, E. P., 1932, Mineralogy of drill cores from the potash field of New Mexico and Texas: U.S. Geological Survey Bulletin $833,124 \mathrm{p}$.

Sheldon, V. P., 1954, Oil production from the Guadalupe Series in Eddy County, New Mexico, in Guidebook of southeastern New Mexico: New Mexico Geological Society, 5th Field Conference, October 21-24, 1954, p. 150159.

Shumard, B. F., 1858, Notice of new fossils from the Permian strata of New Mexico and Texas, collected by Dr. George G. Shumard, geologist for the United States government expedition for obtaining water by means of artesian we1ls along the 32nd parallel, under the direction of Captain John Pope, J.S. Top. Eng.: St. Louis Academy of Science Transactions, V. 1, p. 290-297.

1859, Notice of fossils from the Permian strata of New Mexico, obtained by the United States expedition under Capt. Pope for boring artesian wells along the 32nd parallel, with descriptions of new species from these strata and the coal measures of that region: St. Louis Academy of Sclence Transactions, V. 1, p. 397-403.

Shumard, G. G., 1858, Observations on the geological formations of the country between the Rio Pecos and the Rio Grande, in New Mexico, near the line of the 32nd parallel, being an abstract of a portion of the geological report of the expedition under Capt. John Pope, Corps of Topographical Engineers, U.S. Army, in the year 1855: St. Louis Academy of Science Transactions, v. 1, p. 273-289.

Silver, B. A., and Todd, R. G., 1969, Permian cyclic strata, northern Midland and Delaware Basins, west Texas and southeastern New Mexico: AAPG Bulletin, v. 53, p. 2223-2251.

Skinner, J. W., 1946, Correlation of Permian of west Texas and southeast New Mexico: AAPG Bulletin, v. 30, p. 1857-1874.

Skinner, J. W., and Wilde, G. L., 1954, The fusulinid subfamily Boultonilnae: Journal of Paleontology, v. 28, p. 434-444. 1955, New fusulinids from the Permian of west Texas: Journal of Paleontology, v. 29, p. 927-940.

Smith, D. B., 1973, Geometry and correlation along Permian Capitan Escarpment, New Mexico and Texas: Discussion: AAPG Bulletin, v. 57, p. 940-945. $1974 a$, Origin of tepees in Upper Permian shelf carbonate rocks of Guadalupe Mountains, New Mexico: AAPG Bulletin, v. 58, p. 63-70. 1974b, Sedimentation of Upper Artesia (Guadalupian) cyclic shelf deposits of northern Guadalupe Mountains, New Mexico: AAPG Bulletin, v. 58, p. 1699-1730.

Snider, H. I., 1966, Stratigraphy and associated tectonics of the Upper Permian Cast1le-Salado-Rustler evaporite complex, Delaware Basin, west Texas and southeast New Mexico: Albuquerque, University of New Mexico, unpublished $\mathrm{Ph}$. D. dissertation, $140 \mathrm{p}$.

Spangle, P. F., ed., 1960, A guidebook to Carlsbad Caverns National Park: National Speleological Society Guidebook Series No. 1, 44 p. 
Stafford, P. T., 1959, Geology of part of the Horseshoe atoll in Scurry and Kent Counties, Texas: U.S. Geological Survey Professional Paper 315-A, p. $1-20$.

Stah1, W. J., and Carey, B. D., Jr., 1975, Source rock identification by isotope analysis of natural gases from fields in the Val Verde and Delaware basins, west Texas: Chemical Geology, v. 16, no. 4, p. 257-267.

Steenland, N. C., 1969, Magnetic investigations in the Delaware Basin, in Delaware Basin exploration: West Texas Geological Society, Guidebook, Publicaton No. 68-55a, p. 118-125.

Stipp, T. F., 1952, Surface structures of the foothill region of the Sacramento and Guadalupe Mountains, Chaves, Eddy, Lincoln, and Otero Countles, New Mexico, in Guidebook of south-central New Mexico: Roswell Geological Soclety, Guidebook, 6th Field Trip, 14 p.

Stipp, T. F., and Haigler, L. B., 1956, Preliminary structure contour map of part of southeastern new Mexico showing oil and gas development: U.S. Geological Survey, Oil and Gas Investigations Map OM 177.

Stipp, T. F., and others, eds., 1957, The oil and gas fields of southeastern New Mexico, 1956--a syposium: Roswe11, New Mexico, Roswell Geological Society, $376 \mathrm{p}$.

Strain, W. S., 1969, Cenozoic rocks in the Mesilla and Hueco Bolsons, in Delaware Basin exploration: West Texas Geological Soclety, Guidebook, Publication No. 68-55a, p. 83-84.

Structuremaps., Ltd, 1973, The Permian Basin of west Texas and southeast New Mexico, Permian structure map showing oil and gas production: Midland, Texas, Structuremaps, Limited, scale $1: 348,480,1$ sheet.

Sumnerson, C. H., 1966, Crystal molds in dolomite; their origin and environmental interpretation: Journal of Sedimentary Petrology, v. 36, p. 221-270.

Sweeney, Henry N., ed. in chief, 1961, $0 i 1$ and gas fields of southeastern New Mexico, 1960 supplement--a symposium: Roswe11, New Mexico, Roswe11 Geological Society, 229 p.

Tait, D. B., Ahlen, J. L., Gordon, A., Scott, G. L., Motts, W. S., and Spitler, M. E., 1962, Artesia Group (Upper Permian) of New Mexico and west Texas: AAPG Bulletin, v. 46, p. 504-517.

Tarr, R. S., 1892, Reconnaissance in the Guadalupe Mountains: Texas Geological Survey Bulletin 3, $39 \mathrm{p}$.

Thomas, C. M., 1965, Origin of pisolites (abs.): AAPG Bulletin, v. 49, p. 499.

1968, Vadose pisolites in the Guadalupe and Apache Mountains, west Texas: Permian Basin Section, Society of Economic Paleontologists and Mineralogists, Publication 68-77, p. 32-35.

Thomas, L. C., 1960, Geraldine--Ford field, Culberson and Reeves Counties, Texas, in Geology of the Delaware Basin and field trip guidebook: Midland, West Texas Geological Society, 1960 Field Trip Guidebook, p. 7677.

Thomason, Ben, 1960, E1 Mar field, Loving County, Texas, and Lea County, New Mexico, in Geology of the Delaware Basin and fleld trip guidebook: Midland, West Texas Geological Society, 1960 Field Trip Guidebook, p. 7175.

Thrailkill, J. V., 1971, Carbonate deposition in Carlsbad Caverns: Journal of Geology, v. 79, p. 683-695.

Thrailkill, J. V., and Boyer, P. S., 1965, Occurrence and stability of carbonate minerals in Carlsbad Caverns, New Mexico (abs.): Geological Society of America Program for 1965 Annual Meeting, p. 173. 
Toomey, D. F., and Cys, J. M., 1977, Rock/biotic relationships of the Permian Tansil1-Capitan facies exposed on the north side of the entrance to Dark Canyon, Guadalupe Mountains, southeastern New Mexico, in Hileman, M. E., and Mazzu11o, S. J., eds., Upper Guadalupian facies, Permian reef

complex, Guadalupe Mountains, New Mexico, and west Texas: Permian Basin Section, Society of Economic Paleontologists and Mineralogists, 1977

Field Conference Guidebook (Publication 77-16), v. 1, p. 133-150.

Trollinger, W. V., 1968, Surface evidence of deep structure in the Delaware basin, in Delaware Basin exploration, 1968, Guidebook: West Texas Geological Society Pulication 68-55, p. 87-104.

1969, Surface evidence of deep structure in the Delaware Basin, in Delaware Basin exploration: West Texas Geological Society, Guidebook, Publication No. 68-55a, p. 87-104.

Tyrre11, W. W., Jr., 1962, Petrology and stratigraphy of near-reef Tansi11Lamar strata, Guadalupe Mountains, Texas, and New Mexico, in Wilde, G. L., and others, eds., Permian of the central Guadalupe Mountains, Eddy County, New Mexico: Hobbs, Roswe11 and West Texas Geologica1 Society, Field Trip Guidebook, Publication No. 62-48, p. 59-75.

1964, Petrology and stratigraphy of near reef Tansill-Lamar strata, Guadalupe Mountains, Texas, and New Mexico, in Geology of the Capitan reef complex of the Guadalupe Mountains, Culberson County, Texas, and Eddy County, New Mexico: Roswell Geological Society Guidebook, p. 66-75. 1969, Criteria useful in interpreting environments of unlike but timeequivalent carbonate units (Tansill-Capitan-Lamar), Capitan reef complex, west Texas and New Mexico, in Friedman, G. M, ed., Depositional environments in carbonate rocks: Society of Economic Paleontologists and Mineralogists, Special Publication 14, p. 80-97.

Udden, J. A., 1918, The age of the Castile gypsum and Rustler Springs formation: American Journal of Science, 4th ser., v. 40, p. 151-156. 1924, Laminated anhydrite in Texas: Geological Society of America Bulletin, v. 35, p. 347-354. American Geological Institute, 1958, Geological Abstracts, v. 6, no. 3, p. 51.

Van Der Gracht, W. A. J. M., 1931, The Permo-Carboniferous orogeny of the South-Central United States: Kon. Akademie van Wetenschappen, Amsterdam Vers., Afd. Natuurk., No. 3, deel 27.

Vertrees, C. D., Atchison, C. H., and Evans, G. L., 1959, Paleozoic geology of the Delaware and Val Verde basins, in Geology of the Val Verde basin and field trip guidebook: Midland, Texas, West Texas Geological Society, 1959 Field Trip Guidebook, p. 64-73.

Vertrees, C. D., and others, 1964, Cross-section through Delaware and Val Verde basins from Lea County, New Mexico, to Edwards County, Texas: Midland, Texas, West Texas Geologica1 Society, Publication 64-54.

Vest, E. L., Jr., 1968, Pennsylvanian-Permian horseshoe ato11, west Texas (abs.): AAPG Bulletin, v. 52, no. 3, p. 553.

1970, Oil fields of Pennsylvanian-Permian horseshoe ato11, west Texas, in Halbouty, M. T., ed., Geology of giant petroleum fields--A

symposium: American Association of Petroleum Geologists Memoir 14, p. 185-203.

Vine, J. D., 1960, Recent domal structures in southeastern New Mexico: AAPG Bulletin, v. 44, no. 12, p. 1903-1911. 1963, Surface geology of the Nash Draw quadrangle, Eddy County, New Mexico: U.S. Geological Survey Bulletin 1141-B, 46 p. 
Von Buttlar, H., and Wendt, I., 1958, Ground-water studies in New Mexico using tritium as a tracer: American Geophysical Union Transactions, v. 39, no. 4, p. 660-668,:

Wagner, L. H., Hines, V. J., Thorsen, W. G., and Cys, J. M., 1977, Selected bibliography of the Guadalupian of west Texas and New Mexico, in Hileman, M. E., and Mazzullo, S. J., eds., Jpper Guadalupian facies, Permian reef complex, Guadalupe Mountains, New Mexico and west Texas: Permian Basin Section, Society of Economic Paleontologists and Mineralogists, 1977 Field Conference Guidebook (Publication 77-16), v. 1, p. 500-508.

Watson, W. G., 1974, Inhomogeneities of the Ramsey Member of the Bell Canyon Formation, Geraldine Ford Field, Culberson and Reeves Counties, Texas: Arlington, University of Texas at Arlington, unpublished Master's thesis, $122 \mathrm{p}$.

1979, Inhomogeneities of the Ramsey Member of the Permian Bell Canyon Formation, Geraldine Ford Field, Culberson and Reeves Counties, Texas, in Sullivan, N. M., ed., Guadalupian Delaware Mountain Group of west Texas and southeast New Mexico: Permian Basin Section, Society of Economic Paleontologists and Mineralogists, Symposium and Field Conference Guidebook, Publication 79-18, p. 2-38.

Weinmeister, M. P., 1978, Origin of upper Bell Canyon reservoir sandstones (Guadalupian), El Mar and Paduca fields, southeast New Mexico and west Texas: College Station, Texas $A$ and M University, unpublished Master's thesis, $96 \mathrm{p}$.

West Texas Geological Society, 1951, Introduction to the petroleum geology of the Permian Basin of west Texas and southeastern New Mexico: Midland, Texas, West Texas Geological Society, 51 p.

1960, Geology of the Delaware Basin and field trip guidebook: Midland, Texas, West Texas Geological Society, 1960 Field Trip Guidebook, 97 p. 1966, Oil and gas fields in west Texas--Symposium: Midland, Texas, West Texas Geological Society Publication 66-52, 398 p. 1969a, Delaware Basin exploration: West Texas Geological Society, Guidebook, Publication No. 68-55a, 170 p. 1969b, Oil and gas fields in west Texas--Symposium, Volume 2: West Texas Geological Society Publication 69-57, 134 p.

Whiteman, A. J., 1952, Regressive bioherm theory and Capitan reef: AAPG Bulletin, v. 36, p. 173-175.

Wilde, G. L., 1955, Permian fusulinids of the Guadalupe Mountains: Permian Basin Section, Society of Economic Paleontologists and Mineralogists Guidebook, p. 59-62.

1971, Fusulinacean history and its bearing upon Permian boundary problems (abs.): Bulletin of Canadian Petroleum Geology, v. 19, p. 375376.

1975, Fusulinid-defined Permian stages, in Cys, J. M., and Toomey, D. F., eds., Permian exploration, boundaries, and stratigraphy: West Texas Geological Society and Permian Basin Section, Society of Economic Paleontologists and Mineralogists, p. 67-83.

Wilde, G. L., and Todd, R. G., 1968, Guadalupian biostratigraphy and sedimentation in the Apache Mountains region, west Texas, in Guadalupian facies, Apache Mountains area, west Texas: Permian Basin Section, Society of Economic Paleontologists and Mineralogists, p. 10-31.

Wilkinson, W. M., 1953, Fracturing in Spraberry reservoir, west Texas: AAPG Bulletin, v. 37, no. 2, p. 250-265. 
Williamson, C. R., 1977, Deep-sea channels of the Bell Canyon Formation (Guadalupian), Delaware Basin, Texas-New Mexico, in Hileman, M. E., and Mazzullo, S. J., eds., Jpper Guadalupian facies, Permian reef complex, Guadalupe Mountains, New Mexico, and west Texas: Permian Basin Section, Society of Economic Paleontologists and Mineralogists, 1977 Field Conference Guidebook (Pub1ication 77-16), v. 1, p. 409-432. 1978, Depositional processes, diagenesis and reservoir properties of Permian deep sea sandstones, Bell Canyon Formation, Texas-New Mexico: Texas Petroleum Research Committee, Report no. UT78-2, 260 p. 1979, Deep-sea sedimentation and stratigraphic traps, Bell Canyon Formation (Permian), Delaware Basin, in Sullivan, N. M., ed., Guadalupian Delaware Mountain Group of west Texas and southeast New Mexico: Permian Basin Section, Society of Economic Paleontologists and Mineralogists, 1979 Symposium and Field Conference Guidebook, Publication 79-18, p. 3974.

Willis, Robbin, 1929a, Preliminary correlation of the Texas and New Mexico Permian: AAPG Bulletin, v. 13, p. 907-1031. $1929 b$, Structural development and oil accumulation in Texas Permian: AAPG Bulletin, v. 13, p. 1033-1043.

Wilson, J. H., II, 1960, Twofreds field, Loving, Reeves and Ward Counties, Texas, in Geology of the Delaware Basin and field trip guidebook: Midland, West Texas Geological Society, 1960 Field Trip Guidebook, p. 8184.

Wilson, J. L., 1975, Carbonate facies in geologic history: New York, Springer Verlag, $471 \mathrm{p}$.

Yurewicz, D. A., 1976, Sedimentology, paleoecology, and diagenesis of the massive facies of the lower and middle Capitan Limestone (Permian), Guadalupe Mountains, New Mexico and west Texas: Madison, University of Wisconsin, unpublished $\mathrm{Ph}$. D. dissertation, $278 \mathrm{p}$. 1977, The origin of the massive facies of the lower and middle Capitan Limestone (Permian), Guadalupe Mountains, New Mexico and west Texas, in Hileman, M. E., and Mazzul1o, S. J., eds., Upper Guadalupian facies, Permian reef complex, Guadalupe Mountains, New Mexico and west Texas: Permian Basin Section, Society of Economic Paleontologists and Mineralogists, 1977 Field Conference Guidebook (Publication 77-16), V. 1, p. 45-92. 
PART II

UPPER PALEOZOIC BIOHERMS

IN THE NORTHERN SACRAMENTO MOUNTAINS

ROBERT B. HALLEY 
UPPER PALEOZOIC BIOHERMS

IN THE NORTHERN SACRAMENTO MOUNTAINS

\section{Introduction}

Exposures of Upper Paleozoic strata in the northern Sacramento Mountains offer a superb opportunity to view varied carbonate lithologies, local facies changes, and the products of diagenesis within a variety of shelf and slope carbonate buildups. Similar buildups occur in the subsurface in nearby New Mexico, Texas and Utah basins and are known to be excellent hydrocarbon reservoirs. They have been targets of exploration in the area for the last quarter century.

We will visit three types of mounds and discuss their similarities and differences in the field. On Day IV, we will study phylloid algal mounds, structures which are widespread throughout the United States (Wray, 1968). We will compare a Virgilian mound (Pennsylvanian), which is largely a carbonate mud accumulation, with a Wolfcampian mound (Permian) that contains copious amounts of submarine cement. On Day $\mathrm{V}$, we will visit an Osagean (Mississippian) buildup composed of a muddy core facies and crinoidal sand flank beds. This mound is similar to Lower Carboniferous mounds of Europe, known as Waulsortian Mounds and named from occurrences near Waulsort, Belgium.

The exposures provide a cross-sectional view of the rocks, but it is not possible to develop a regional picture of facies realtionships in a few days as may be done for the Permian Basin. The Permian part of this field course visits an area where erosion and evaporite solution produced outcrops that may be re1atively easily related to a paleogeographic framework. In contrast, strata in the northern Sacramento Mountains dip into the subsurface a few miles to the east of the outcrops, and they are downfaulted below the Tularosa Valley to the west. 
The northern Sacramento Mountain area was closer to sources of terrigenous clastic sediments than the Carlsbad area during the late Paleozoic. The Pedernal land mass repeatedly shed material south and west to the Alamogordo area. Some of the tectonism which occurred during this time is evidenced in the Sacramento Mountains by Late Paleozoic faulting. Some tectonism may also be reflected in the sediments themselves, which show evidence of repeated, relative sea level changes, probably of both tectonic and eustatic origin.

In the northern Sacramento Mountains we will continue to investigate many of the themes developed in the Carlsbad area, but now in a considerably different setting. These themes include facies relationships, faunal and lithologic variation, reef models, marine cementation, subaerial exposure, porosity and permeability development and preservation. They are themes which are increasingly incorporated into modern exploration scenarios and are well illustrated by the outcrops we will visit.

Summary of Significance to Petroleum Exploration

The general geology of the northern Sacramento Mountains has been worked out by Pray (1952, 1961) and Otte (1959b), who provided the framework for many later, more detailed studies. Pray (1959) summarizes work in the area before 1950. Excellent general field guides to the area have been published by Pray (1959) and Butler (1977). Pray (1975) has recently edited a field guide to shelf-edge and basin facies limestones in the Sacramento Mountains. Figure 1 indicates the position of our field stops on a generalized stratigraphic section for the northern Sacramento Mountains.

Several processes discussed and developed at outcrops in the Permian Reef complex will again be evoked to explain observations on these older bioherms. The significance of these processes varies from buildup to buildup, and the internal structure and composition of the bioherms reflect these differences. Some buildups are cement-rich, some mud-rich, some contain shallow-water fossils, some deep-water 


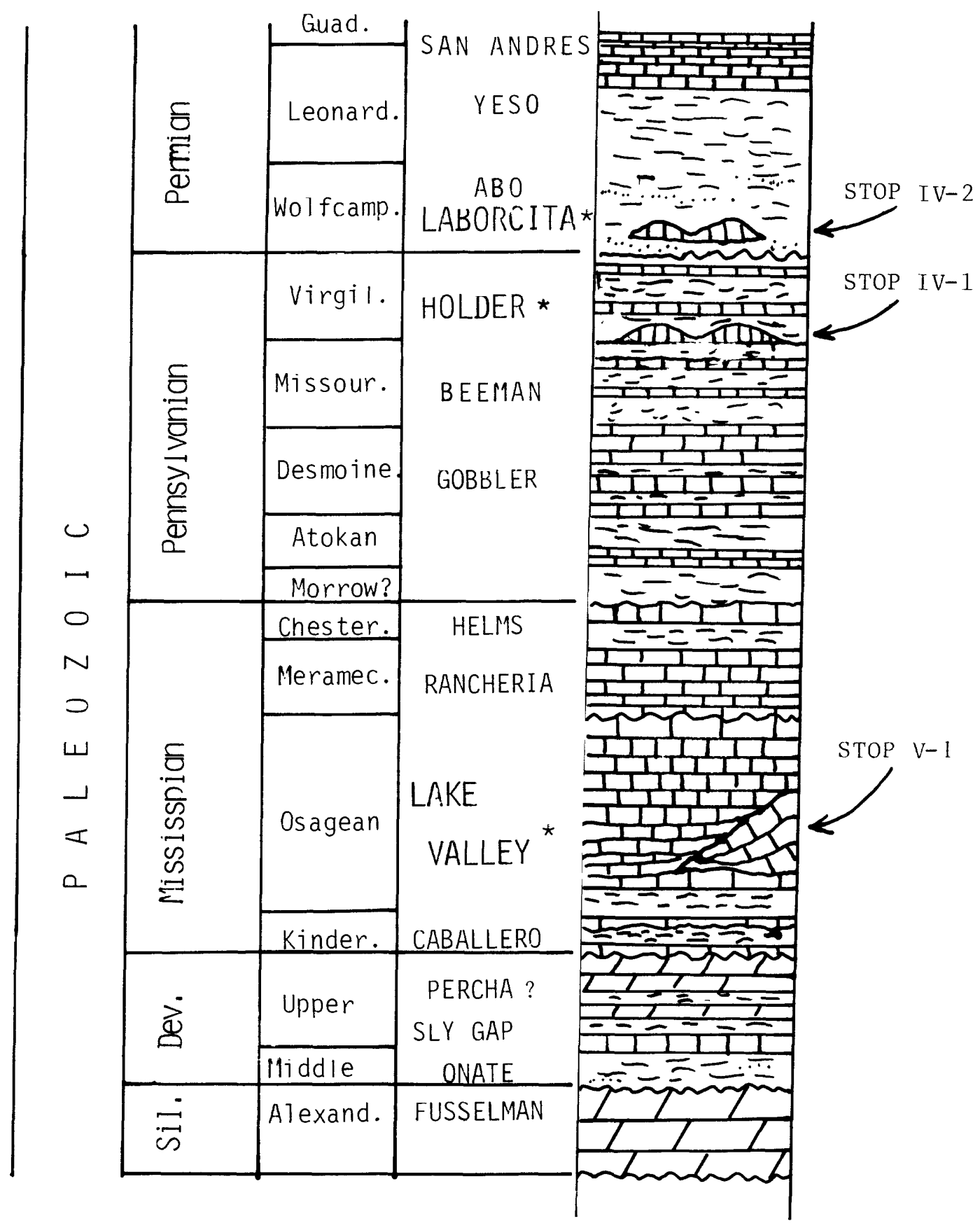

Figure 1. Partial stratigraphic column of the northern Sacramento Mountains showing field trip stops. Section is generalized after Pray (1961) and AAPG Geologic Road Map, Eastern Colorado and New Mexico. 
fossils. We will try to extract as much interpretive data as possible from bioherm outcrops. Such observations will help to interpret similar rocks in the subsurface. In contrast to the Capitan Reef, which does not produce oil in the subsurface, bioherms similar to those we visit in the Sacramento Mountains do form excellent reservoirs. Phylloid algal limestones, like those at Virgil and Yucca mounds, form reservoir rocks at Aneth Field (Elias, 1963; Irwin, 1963; Peterson and Ohlen, 1963), Ismay Field (Choquette and Traut, 1963), New Lucia Field (Toomey and Winland, 1973), Lusk Field (Thornton and Gaston, 1968), several fields in the "Horseshoe Ato11" (Vest, 1970), and Saunders and Conley fields (Kerr, 1969). These studies show, in some cases, direct association of subsurface porosity and the presence of phy110id algae. Porosity takes the form of shelter pores beneath algal blades in mudstones and wackestones, intergranular porosity in algal plate grainstones, and secondary porosity in leached algal plate mudstones. In some cases porosity and permeability are provided by fracturing or dolomitization in this facies. Several of the associated 1ithologies also provide excellent reservoir rock, some of which are oolitic, crinoidal and fusulinid grainstones. It is significant that production from many fields appears to be from the shelf-edge buildups themselves and not from fore-reef or back-reef facies. Fields along the Abo Trend (LeMay, 1972) and the KemnitzTownsend Trend (Malek-Aslani, 1970) occur at the shelf edge, a position occupied by the tight Capitan Limestone in younger units to the south. Early submarine cementation is a major factor in the lack of oil production from the Capitan reef. The retention of porosity in the subsurface is still a topic of considerable study. We see little matrix porosity in outcrops of Late Paleozoic mounds (although vugs are characteristic of the lower Virgil Mound). The original porosity in these carbonate sediments was very high (40-85\%), and the processes involved in such great porosity loss have not been well documented. One of the best documented cases of porosity loss in carbonate sands comes from studies of the crinoidal sand of the Lake Va1ley Formation. 
Hydrocarbon reservoirs in rocks similar to those that occur in the Lake Valley appear to be rare. Pray (1958) reported that cores from the La Pan Field of Clay County, Texas, are similar to lithologies associated with Muleshoe Bioherm. La Pan Field may therefore be a buildup similar to Muleshoe Bioherm. Meyers (1974) showed that cementation and porosity loss in Lake Valley non-biohermal sediments are linked to periods of subaerial exposure. As much as $60 \%$ of the original porosity was lost within about five million years of sediment deposition. Almost a11 the rest was lost within 20-30 million years. Cementation took place during two episodes of subaerial exposure (Meyers, 1978).

It is interesting to note that early subaerial exposure is credited with producing leached porosity in many phylloid algal mounds (Wilson, 1975). Apparently, exposure and fresh-water diagenesis act as a double-edged sword, i.e., under some circumstances exposure helps produce reservoir rocks; in other cases exposure destroys reservoir properties. The particular circumstances which control the products of exposure are not well understood. Factors that probably exert considerable influence on the diagenetic history of these rocks include rate of transgression or regression, duration of exposure, climate, original sediment mineralogy and local paleohydrology.

Even less well understood are later diagenetic processes which may affect these limestones in the subsurface. The Holder and Laborcita mounds had been buried to at least 2500 feet and the Lake Valley bioherms as deeply as 6500 feet by the end of the Paleozoic. Processes, such as compaction, pressure-solution cementation, fracturing, cementation by dolomite and anhydrite, are known to occur at depth but are undocumented in these rocks.

Finally, one wonders what has been the effect of uplift and the current episode of exposure on these rocks. Might some of the differences between the rocks we see in outcrop and their subsurface counterparts be due to their Cenozolc uncovering? 
Or was the character of these rocks essentially fixed during their burial? Again, it should be emphasized that we will not develop a regional picture of sedimentary facies in the Sacramento Mountains as we do in the Guadalupe Mountains. Generalized paleogeographic maps for the Osagian and Virgilian Stages of the area are outlined in Figure 2 . We will review principles of carbonate deposition as they apply to late Paleozoic bioherms and formulate new questions which have particular significance to petroleum exploration. 

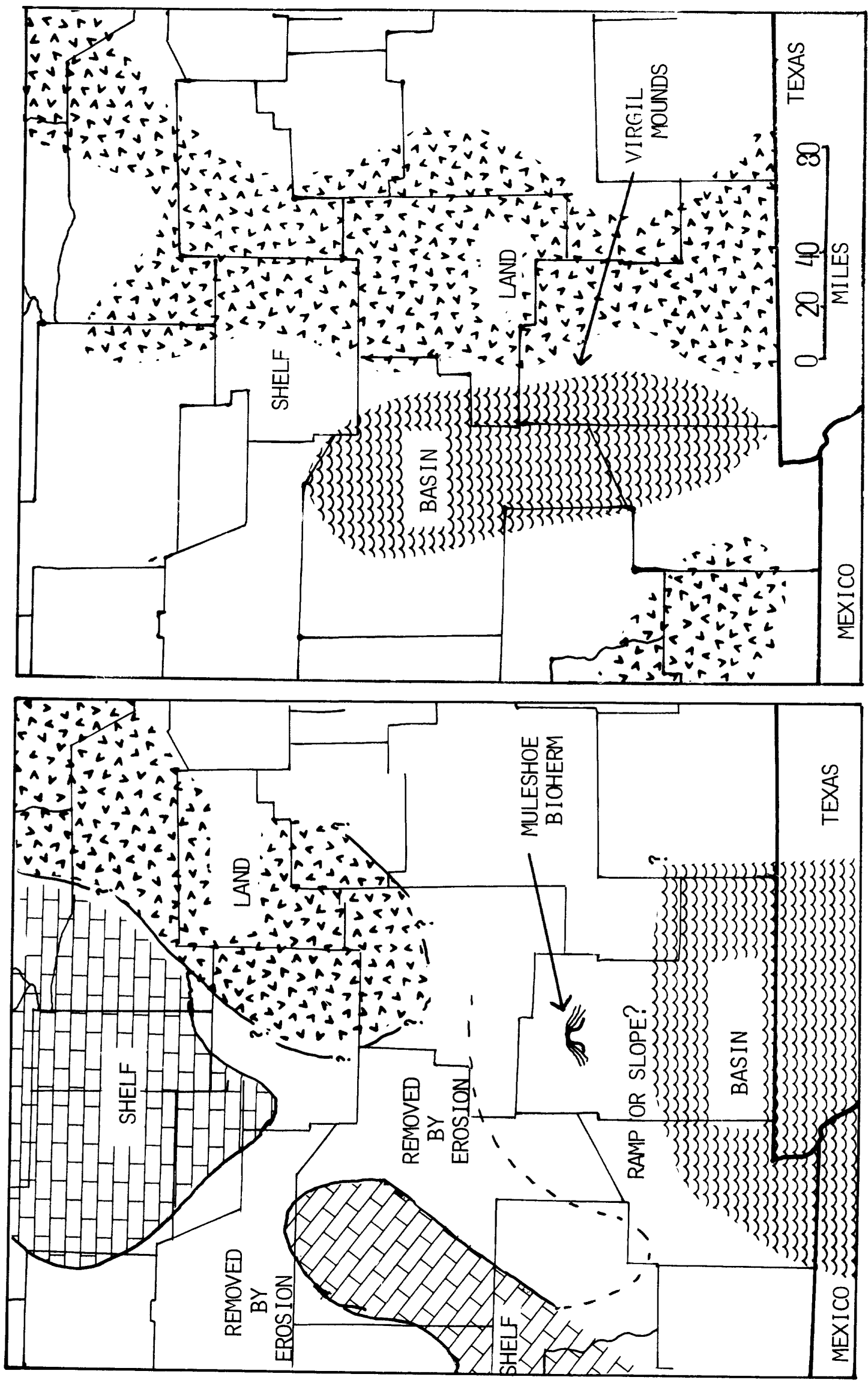

$ص$

先

出䡒 롬ำ

ขึํำ

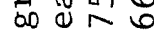

- 0 a

क 007

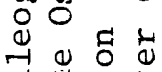

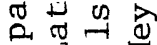

, 留

$\stackrel{N}{*} \mathbb{Z}$

-

क $\infty$

동

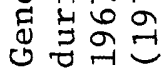

$\leftarrow \quad \dot{v}$

岁

$\cdot \rightarrow$ 
CARLSBAD TO ALAMOGORDO ROADLOG

DAY IV

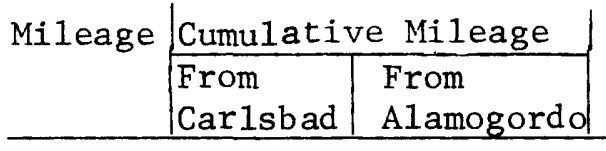

Description

$0.0 \quad 0.0 \quad 181.4 \quad$ Leave Carlsbad traveling north on US 285 from its junction with US 62-180 in downtown Carlsbad traveling toward Artesia, N.M.

$21.321 .3 \quad 160.1$ Junction with NM 137. Continue north on US 285. As we travel northward, we cross several "reef" zones in the subsurface. Just a few miles north of here, our route crosses the trend of the San Andres reef zone (Miller, 1969) and further to the north, we cross the Abo reef trend about 5 miles south of Artesia (LeMay, 1972). In general, these trends parallel the Capitan Reef trend in the subsurface along the northern end of the Delaware Basin.

$0.8-\quad 22.1-\quad 159.3-$

$2.8 \quad 24.1 \quad 157.3$

$\begin{array}{lll}5.6 & 29.7 & 151.7\end{array}$

$9.4 \quad 39.1 \quad 142.3$

$4.1 \quad 43.2 \quad 138.2$

$\begin{array}{lll}1.5 & 44.7 & 136.7\end{array}$

$\begin{array}{ccc}21.3 & 66.0 & 115.4 \\ 6.9 & 72.9 & 108.5 \\ 5.7 & 78.6 & 102.8 \\ 0.2 & 78.8 & 102.6 \\ 0.3- & 79.1- & 102.3- \\ 13.3 & 92.1 & 89.3 \\ 1.7 & 93.8 & 87.6\end{array}$

Low roadcuts through thin-bedded dolomites (with evaporite crystal casts) and redbeds of the restricted lagoon facies of the Yates and Seven Sisters Formations, similar to outcrops visited in Dark Canyon and Rocky Arroyo on Day III.

Junction with NM 381. Hills visible 6 miles to the east form the McMillan Escarpment and are composed of the Permian Artesia Group (undivided Tansil1, Yates and Seven Sisters Fms.).

Junction with NM 335, continue north on US 285 toward Artesia.

Entering Artesia, N.M.

Turn left at junction with US 82 (US 83 on maps published prior to 1968) traveling west toward Cloudcroft. Road traverses 20 miles of Quaternary alluvium, good time for a nap or reading detailed descriptions of future stops. Hope Village center. Chaves County line. Junction with NM 13, continue west on US 82 . Roadcut in Quaternary alluvium.

Scattered outcrops of dark grainstones of the San Andres Formation.

Junction with NM 24 on left. Continue on US 82 west into foothills on the east side of the Sacramento Mountains. Hillsides and roadcuts for the next 45 miles are either 11mestones of the San Andres Fm. or red and yellow terrigenous clastics of the Yeso Fm. US 82 slowly climbs the east side of the Sacramento Mts. (almost a dip slope), gradually cutting deeper into the San Andres limestones and into the Yeso below. As elevation Increases, we will pass through several vegetation zones, from open desert. scrub to alpine conifer forest.

Village of Elk, N.M.

Mule Canyon Road on left.

Otero County line.

Enter Lincoln National Forest.

Mayhill town limit. For the next $10 \mathrm{miles}$, valley is floored by the Yeso Fm., and the San Andres Fm. occurs on the surrounding higher areas. 


\begin{tabular}{|c|c|c|}
\hline 0.4 & 118.5 & 62.9 \\
\hline 10.3 & 128.8 & 52.6 \\
\hline 1.1 & 129.9 & 51.5 \\
\hline 4.1 & 134.0 & 47.4 \\
\hline 1.9 & 135.9 & 45.5 \\
\hline 1.3 & 137.2 & 44.2 \\
\hline 0.2 & 137.4 & 44.0 \\
\hline 0.2 & 137.6 & 43.8 \\
\hline 1.7 & 139.3 & 42.1 \\
\hline 1.5 & 140.8 & 40.6 \\
\hline 3.2 & 144.0 & 37.4 \\
\hline 0.6 & 144.6 & 36.8 \\
\hline 0.9 & 145.5 & 35.9 \\
\hline 1.0 & 146.5 & 34.9 \\
\hline 0.3 & 146.8 & 34.6 \\
\hline & igure 3 & \\
\hline 0.7 & 147.5 & 33.9 \\
\hline 0.8 & 148.3 & 33.1 \\
\hline 0.8 & 149.1 & 32.3 \\
\hline 0.5 & 149.6 & 31.7 \\
\hline 0.7 & 150.3 & 31.1 \\
\hline
\end{tabular}

Figures 4

and 5
Junction with NM 130 on left.

Junction with Springs Canyon Road on right.

Entering town of Winsatt, N.M.

Cloudcroft Ski Area on left.

Junction with NM 24 on right.

Cloudcroft town center, elevation about 8700 feet. US 82 drops rapidly, some 4000 feet in the next 20 miles, down the west face of the Sacramento Mts.

Alluvium exposed in roadcut to left and right.

Lower San Andres Fm.

Yeso Fm. on right. US 82 drops through over 1200 feet of Yeso $\mathrm{Fm}$. in next 5 miles.

Lower Yeso redbeds typical of the next 3 miles.

Gradational contact of Yeso Fm. (marine) with underlying

Abo Fm. (nonmarine) occurs in this general area.

Mountain Park, N.M.

High Rolls, N.M. and junction with West Side Road. High

Rolls is a lead and copper mining district with ores

occurring in arkose beds of the Abo Fm. (Jerome and others, 1965).

East side of tunnel and Fresnal Box Canyon walls composed of the Bug Scuffle Limestone Member of the Gobbler Fm. (Middle Pennsylvanian). On this side (east side) of the Fresnal Fault, the Laborcita, Holder and Beeman Formations are missing, but they are present on the west side of the fault.

OPTIONAL STOP. Fresnal Box Canyon vista on right. Effects of drag from the Fresnal Fault Zone on Bug Scuffle Ls. Member may be viewed to the west on the north side of the canyon. Fresnal faults in this area were active during the latest Pennsylvanian and earliest Permian. Delgado and Pray (1977) estimate as much as 1600 feet of displacement, down to the west, along the fault zone in this area. Dry Canyon ahead and to the left (south).

Tertiary dike and sill intruding Laborcita Fm. on right. Covered, unconformable contact between Laborcita and Holder Fms. at approximately this point.

View ahead of flank beds dipping eastward off Virgil bioherms.

STOP IV-1, Virgil Bioherm. Lincoln National Forest boundary sign and parking area on left. The west flank of the bioherm is strikingly exposed to the north of the road. Beds with apparent dips of almost $45^{\circ}$ at the west end give way to less steeply dipping strata and massive units in the center of the bioherm outcrop. Faint bedding near the center suggests the escarpment does not expose core facies or that the bioherm core is very similar to flanking beds. The 60-80-ft.-thick feature is typical of many such bioherms in the area and shares features in common with many late Paleozoic buildups.

Plumley and Graves (1953) first figured the Virgilian bioherms and emphasized their geometry, orientation and biological origin. They appear as elongate bodies up to one mile long and 200 feet thick. They tend to parallel the mountain front. As we traverse the outcrop here, it 
will become apparent that the bioherm, while beautifully exposed, does not weather in a manner that allows easy interpretation of the composition of these limestones. Parks (1958, 1962), Wray $(1959,1963)$ and Konishi and Wray (1961) established the importance of platy algae in these limestones and have refined the taxonomy of the platy or phylloid (leaf-shaped) algae (Pray and Wray, 1963). Phylloid algal limestones are widespread in the United States (Wray, 1968), and bioherms composed of such algal limestones are widespread in Late Pennsylvanian and Wolfcampian strata of southern New Mexico (Wilson, 1977). Cline (1959) emphasized the cyclic nature of Virgilian rocks in this area, and Wilson (1967) related these shelf cycles to basinal cycles in the Orogrande Basin. Shelf cycles consist of a variable sandstone and shale lower member with local channel-fill conglomerates which grade upward into normal marine limestone and shale. These in turn pass upward into shallow-water limestones (grainstones and bioherms) which cap the shelf cycles. Wilson (1967) interpreted these cycles to be the result of repeated sea level fluctuations, which periodically exposed the shelf and bioherms to subaerial weathering and diagenesis. Wilson drew on conceptual models of cyclic deposition and evidence from the Holder Fm. to develop the idea of shelf and basin reciprocal sedimentation. This important model promotes alternate sites of basin and shelf sedimentation during sea level low and high stands, respectively. During lowered sea level, most sediment bypasses the exposed shelves and is deposited in adjacent basins. During sea level high stands, these sediments are deposited on the shalves along with shallowwater limestones, while the basins recelve little sediment and are "starved." The reciprocal sedimentation concept has been widely applied in shelf/basin sediment dynamics, and we will discuss its application to the Permian Basin.

Pray (1961) defined the La Luz anticline, which runs approximately NNW through this area and had a pronounced local influence on Late Pennsylvanian sedimentation (Wilson, 1969). Bioherms developed with long axes approximately parallel to the axis of the La Luz anticline, which was a subtle structural feature at the time but developed more strongly during later deposition of the Holder Formation.

Inspection of the bioherm proceeds up a gully around the west end of the feature, up-flanking beds eastward to the top of the mound. Before reaching the mound proper, the climb traverses marine shales and limestones of the upper Beeman Formation and enters the Holder Formation approximately 60 feet below the base of the bioherm. The limestones include oolitic and algal grainstones and algal boundstones separated by slope-forming shales.

The bioherm is composed in large part of mud (micrite) and phylloid algal plates. The phylloid algae are usually poorly preserved as molds or replaced by blocky calcite with all traces of original microstructure lost. They 
are probably of diverse origin, some being green calcareous algae and some being red calcareous algae. Their poor state of preservation suggests they were originally aragonitic. Wray $(1975,1977)$ has suggested that the closest living analogues to some of the phylloid algae may be a family of red calcareous algae known as the Squamariacean algae. Today, "squamies" are subtle but widespread coral reef inhabitants.

A great many other organisms are evident in the biohermal rocks, including stromatolites, sponges, tubular encrusting foraminifera, stromatoporoids, and corals, all of which are capable of producing reef structures, but none of which appear to be abundant enough on outcrop to account for the bulk of the carbonate buildup. Volumetrically, mud is the most important constituent of the bioherm. Wray (1959) suggests the mud had been trapped by a thicket of phylloid algae, probably in a relatively low-energy setting. Ball and others (1977) question the mound-building capabilities of phylloid algae. Parks (1977) briefly reported that algal plate mudstones were uncommon in four cores taken through the bioherm. The upper bioherm contained calcirudites of sponge, stromatoporoid, tubular foraminifera, and other clasts. The lower part of the mound contained more mud and calcarenite and rare-to-abundant masses of fibrous calcite. Earlier outcrop studies by otte and Parks (1963) suggest $30-50 \%$ of the lowest third of the bioherm is composed of botryoidal fibrous calcite, a replacement after aragonite submarine cement. The material is beautifully illustrated by the authors and was interpreted as fossil remains of a Stromatactis-like organism, following similar interpretations of fibrous cements from Europe. Otte and Parks (1963) point out that fibrous calcites weather indistinctly in the Virgil reef and are difficult to observe in outcrop, but are strikingly accentuated by weathering at Stop IV-3.

The lower third of the bioherm is also vuggy in outcrop with irregular voids up to several inches across scattered throughout the rocks. The origin of these vugs is problematic. Parks (1977) considered several mechanisms for producing the vugs, including subaerial solution (Wilson, 1975), submarine solution, decay of pre-existing soft-bodied organisms, sheltered parosity, dewatering contraction and gas bubbles. He concluded that vugs were the product of a combination of decay and gas generation.

Small fractures and in situ brecciation (compactional) are common in some portions of the bioherm and are evidence of early lithification. Wilson (1975) suggests this early cementation and vuggy leaching took place during a sea level low stand and are the result of early meteoric water diagenesis. This explanation seems particularly likely in light of the cyclic nature of Holder sediments overlying the bioherm and the transgression/regression model that explains their origin (Wilson, 1967).

The abundant evidence of submarine cement illustrated by Otte and Parks (1963) and Parks (1977) suggests that early submarine cement should be considered as the lithifying 


\section{$0.3 \quad 150.6 \quad 30.8$}

$0.3 \quad 150.9 \quad 30.5$

Figure 6 agent in these buildups. Less obvious micritic submarine cements may also be present in the bioherm sediments, cements which appear identical to detrital micrite. Such cements are common in Holocene reefs, are composed of high-Mg calcite, and appear as a micritic matrix in the reef rock (Macintyre, 1977). It is intriguing to imagine what role submarine cement may have had in creating vuggy porosity in these reefs. In a sense, portions of the bioherm are really "lithoherms," following the terminology used by Neumann and others (1977) to emphasize the role of subsea cementation during growth of the structure. This role is nicely illustrated by substituting submarine cement for "Stromatactis" and quoting from Otte and Parks (1963), "(Submarine cement) functioned as both a sedimentbinding and a framework-building organism in the construction of the bioherms and may be quite widespread in other late Paleozoic bioherms of the western United States."

This has proven to be a rather prophetic statement in light of the now widely recognized submarine cements present in reefs of all ages.

Cattle guard in road and view straight ahead of the Tularosa Basin. In the basin, to the south, lie the outskirts of Alamogordo and the dunes of White Sands National Monument beyond. Across the basin rise the San Andres Mts. Pray (1959) estimates as much as 7000 feet of vertical displacement has occurred along the faults which formed the front of the Sacramento Mts.

OPTIONAL STOP, Yucca Mound. An unused dirt trail leads north of US 82 about 300 yards until it intersects the gully to the right (east). Route turns up gully at intersection until Yucca Mound is reached, about 100 yards after leaving the trail. The beds exposed in the gully below the mound and the mound itself have been studied in considerable detail by Toomey and others (1977a, 1977b).

Exposed in the gully bottom are beds interpreted to represent facies which are basinward and slightly older than the mounds. These include shales, some of which show evidence of penecontemporaneous deformation (slumping?), crossbedded grainstones composed of material presumably transported from mound areas (e.g., sands composed almost entirely of fragments of tubular foraminifera), and small mounds (12-15 feet in diameter) composed of plumose algae and foraminifera.

The core facies of the mound is well exposed and consists of mud and algal plates, and a great variety of other fossils, including sponges, foraminifera, pelecypods, and dasyclad (calcareous green) algae.

Mound geometry can be seen by climbing the steep slope on the north side of the gully and looking south across the canyon that transects the mound. Beds immediately above the mound are nearly horizontal on top of and east of the buildup, but dip steeply over the western edge.

The bioherm is a complex of two mounds, as pointed out by Toomey and others (1977b), and in detail includes a complex variety of carbonate lithologies. One mound overlies and 


$\begin{array}{lll}3.2 & 154.1 & 27.3 \\ 2.2 & 156.3 & 25.1 \\ & & \\ & & \\ & & \\ & & \\ & & \\ & & \\ 7.0 & 163.3 & 18.1 \\ 0.6 & 163.9 & 17.5 \\ 0.8 & 164.7 & 16.7 \\ 0.3 & 165.0 & 16.4 \\ 0.5 & 165.5 & 15.9 \\ 0.9 & 166.4 & 15.0 \\ & & \\ 0.1 & 166.5 & 14.9\end{array}$

Figure 7 is basinward of the other, showing that the complex as a whole built seaward and is regressional in character. However, the presence of stratigraphic breaks in the complex, interpreted to be of subaerial origin, emphasize the complex history which gave rise to this generally regressive sequence. Toomey and others (1977b) estimate the position of the mound to be $\frac{1}{2}$ mile east of the shelf edge. The shelf at this point was narrow (a few miles?) with the Orogrande Basin to the west and the Pedernal Uplift to the east.

Submarine cements have not been identified in the Yucca Mound and appear not to have been important in mound development here.

Junction of US 82 with US 54-70. Turn right (north) toward Tularosa.

Junction with NM 545. Continue straight ahead. The town high on the alluvial fan two miles to the east is La Luz, at the mouth of La Luz Canyon. The strata exposed in the escarpment of the Sacramento Mts. dip to the north, so that increasingly younger beds lie at the base of the mountains to our right as we approach Tularosa. East of Alamogordo, these beds are Mississippian or older in age. At the last stop and east of us now, they are Pennsylvanian, and east of Tularosa they will be Permian in age.

Tularosa city limits.

Bear east (right) on US 70 toward Roswell.

Turn left (north) onto Bookout Road.

Cross bridge over canal.

Right turn onto Bookout NE (east).

Sharp right turn (south) in road, park at safe distance around turn.

STOP IV-2, Laborcita Lithoherms. Hilltops $\frac{1}{4}$ mile to the $\mathrm{NE}$ are capped by strikingly banded limestones of Wolfcampian age. From this distance, it can be seen that the dark bands (cement-rich) pinch and swell along the face of the outcrop. In contrast, the light bands (mud-rich) are of rather even thickness and drape over the topography of the dark bands. This is strong evidence for the excellent mound-building capabilities of cement. Walk to the lithoherms via a meandering route over beds of the Laborcita Formation of Otte (1959a, 1959b). Here, these beds include red and green sandstones, siltstones and mudstones with some spectacular polymict conglomerates and thin limestones. The lithoherms have been described in increasing detail by Otte (1954, 1959a, 1959b), Otte and Parks (1963), Cys and Mazzullo (1977), and Mazzullo and Cys (1979). Because of their proximity to underlying nonmarine beds, the lithoherms are thought to be nearshore marine buildups, perhaps analogous to fringing reefs. Otte (1959) estimates the lithoherms to have stood as much as 60 feet above the surrounding bottom. Again, we use the term "1ithoherm" (Neumann and others, 1977) to describe these mounds in order to emphasize the inferred role of submarine cement in mound development.

Weathering of these mounds has left internal structure easily visible. A number of components may be readily recognised 
Figure 8

$12.4 \quad 178.9 \quad 2.5$

$2.5 \quad 181.4 \quad 0.0$ in outcrop. These include: (1) grey, commonly well laminated lime mud; (2) dark calcite cement, which fractures to reveal a blocky structure but may be viewed in low angle light to reveal a relict fibrous habit; (3) phylloid algal fragments; (4) fractures; (5) scattered sand pockets, some graded; (6) some coarsely crystalline, white, pore-filling calcite, and (7) brown dolomite. The dark calcite cement derives its color from submicroscopic inclusions of organic matter, which is apparently not extractable (Plumley and Graves, 1963). On outcrop, some areas of the 1ithoherms may be seen to be extremely rich in this dark cement. In places, it forms an anastomosing network with lightercolored sediments infilling voids within the cement framework. Cys and Mazzu11o (1977) and Mazzu11o and Cys (1979) estimate this cement to account for $50-85 \%$ of the mound volume. They interpret the cement to be a marine cement that grew on the sea floor and within voids in the mound. It is interesting to compare these figures with estimates of $30-40 \%$ in-place coral in modern and Pleistocene coral reefs. It would appear that there is considerably more cement framework in the Laborcita lithoherms than there is coral framework in many coral reefs.

At several locations along the exposure of the lithoherm, the contact between a dark, cement-rich layer and a lighter, mud-rich layer may be observed in considerable detail at close range. Note that this contact is not erosional (and that the relief on the cement-rich bands is not erosiona1), but rather the contact appears to be a sharp change in the character of sedimentation. It is quite clear from these outcrop relationships that it is the marine cement in these mounds that has been responsible for their reef-like growth. These exposures provide the best evidence, in the writers' opinions, of ancient examples of lithoherms, and they are certainly the most extreme case of submarine cementation in mounds that we will observe on this trip.

Turn around and retrace route backwards to junction of US $54-70$ with US 82 .

Junction of US 54-70 with US 82 . Continue straight ahead toward Alamogordo.

Junction of US 54-70 with 10th Street, Alamogordo, N.M. 


\begin{tabular}{|c|c|c|}
\hline \multirow[t]{2}{*}{ Mileage } & Cumulative & Mile \\
\hline & $\begin{array}{l}\text { From } \\
\text { Alamogordo }\end{array}$ & $\begin{array}{l}\text { From } \\
\text { E1 P }\end{array}$ \\
\hline 0.0 & 0.0 & 96.9 \\
\hline 1.9 & 1.9 & 95.0 \\
\hline 0.4 & 2.3 & 94.6 \\
\hline 4.8 & 7.1 & 89.8 \\
\hline 2.0 & 9.1 & 87.8 \\
\hline 0.1 & 9.2 & 87.7 \\
\hline 0.9 & 10.1 & 86.8 \\
\hline 0.4 & 10.5 & 86.4 \\
\hline 0.7 & 11.2 & 85.7 \\
\hline 0.3 & 11.5 & 85.4 \\
\hline 0.4 & 11.9 & 85.0 \\
\hline
\end{tabular}

Figure 9

Leave Alamogordo traveling south on US 54-70 from junction
with 10 th Street.

Railroad overpass, merge left. Turn left on US 54 to E1 Paso.

Turn left on ranch road (dirt) over railroad tracks and stop to observe Muleshoe Bioherm.

Turn right (south) at " $\mathrm{T}$ " in road.

Road to left leads to Donald Taylor Ranch house and permission should be requested to travel ranch roads to the

foot of the mountains.
Turn left (east) on well graded road leading up alluvial fan toward the mouth of San Andres Canyon.

End of well graded road. Busses and rental cars must stop here. Trip continues via 4-wheel-drive vehicles.

Continue east on trail approximately 0.6 miles to mouth of San Andres Canyon. At canyon mouth, turn left on old road that leads downslope to the northwest.

old corral ahead on left. Find even older road which leads to the right (north) toward Muleshoe Bioherm.

End of old ranch road and mouth of Muleshoe Canyon. Begin approach to Muleshoe Bioherm from here on foot. STOP V-1, Muleshoe Bioherm. At the start of the walk, we may observe the arched appearance of eroded flank beds on the southwest side of the bioherm. Below this arch is exposed a small portion of core facies that will be our final objective. From the entrance to Muleshoe Canyon, route travels up the wash about 200 yards, then begins to climb out of the wash on the north side of the canyon, angling eastward toward a point that will bring us to the same elevation as the base of the bioherm but several hundred yards east of it and standing on non-biohermal sediments. The approach to the bioherm climbs out of the wash over Silurian and Devonian rocks of the Fusselman, Onate, Sly Gap and Percha(?) Formations. About 60 feet of Mississippian Caballero Formation unconformably overlies the Devonian and underlies the biohermal Lake Valley Formation. On reaching an elevation equal to that of the base of the bioherm escarpment and a position about onethird of a mile east of the bioherm, we can observe typical interbioherm lithologies of the Lake Valley Formation. From this point, a westward traverse along the base of the bioherm leads us from normal horizontallybedded Lake Valley sediments into increasingly steeply dipping biohermal flank beds, blocks of core rubble, and finally into bioherm core facies beneath the "arch" viewed earlier from below.

The bioherm has been studied in increasing detail for over 40 years with general descriptions by Laudon and Bowsher (1941, 1949), who subdivided the Lake Valley Formation into six members. Muleshoe Bioherm occurs in the second, third and forth members from the base of the Lake Valley, rises above the last two members of the Lake Valley and above the level of the overlying Rancheria Formation and protrudes 
into the base of overlying Pennsylvanian deposits of the Gobbler Formation (Pray, 1958, 1961). Bioherms about five miles to the north are decidedly elongate in a northsouth direction and are not as thick as Muleshoe Bioherm. Muleshoe is estimated to have stood more than 300 feet above the general bottom level and may have developed on a relatively deep portion of a shelf that became more sha1low to the north. Armstrong (1962, 1967) has suggested that a starved basin lay to the south, and tidal flat deposits are found 160 miles to the north. Land formed by the Pedernal Uplift lay about 80 miles northeast. Muleshoe Bioherm appears almost circular in plan and may have formed in deeper water than the bioherms to the north. Wilson (1975) stated that most geologists acquainted with Mississippian bioherms believe they accumulated below wave base and perhaps below the photic zone. Again, it is tempting to make comparisons between these bioherms and the lithoherms of the Straights of Florida described by Neumann and others (1977).

Note that the faunal components of the bioherm did not require light for their survival. The common forms we will see in the flanking beds are crinoids, brachiopods, bryozoans, and solitary corals. In contrast to other bioherms observed on this trip, calcareous algae are absent. Other typically shallow-water forms, such as massive corals, clams and calcareous sponges, are rare or absent. The crinoidal grainstones of the flank beds are poorly sorted and contain articulated columns several inches long, which suggest flanking crinoidal beds formed in close proximity

Figure 10 to where the crinoids lived. These biogenic sediments appear not to have been transported far from their source. As we approach the core facies of the bioherm, the slope of these flank beds increases to nearly $40^{\circ}$.

The core facies has been studied in great detail by Pray (1958, 1965a, 1965b, 1969) and Lohmann and Meyers (1977). Approximately two-thirds of the core consists of mud. The major faunal component is fenestrate bryozoa. Local cement-rich pockets in the core facies contain bryozoan fragments coated with banded, isopachous cement, which is cloudy when viewed in thin section. This cement was interpreted as marine in origin by Pray (1965a, 1965b). The cloudy cements contain inclusions of microdolomite, as illustrated by Lohmann and Meyers (1977), and are believed to be diagenetically altered high-Mg calcite marine cement. The origin of Muleshoe Bioherm and similar Lower Carboniferous mud mounds had been the topic of considerable conjecture. Are the muddy cores the result of currents piling up fine-grained sediment? The circular plan view of some mounds would make this possibility unlikely. How much sediment may have been trapped or baffled by bryozoan fronds or crinoid thickets? What was the source of the mud? Why are the flank beds so distinct and sharply separate from the core facies? What role did submarine cement play in building these mounds? Many of these questions cannot be answered very satisfactorily. Volumetrically, cement is not nearly as important in the core facies of 
Muleshoe Bioherm as it is in the Laborcita lithoherms. Submarine cement by itself did not build Muleshoe Bioherm, but it would take only a small amount to act as a binding agent to hold the core facies together. If this were the case, then the next question is why did the cement form here, localized in this mound? The sandy flank beds evoke clear visions of crinoidal meadows on the sides of the bioherm, but why not on the top or in the center, where the core facies predominates? Was the central position of the bioherm dominated by some mud-producing organism that decayed so completely that no trace is left behind? Or, was the center isolated from nutrientrich currents, which fed animals on the sides of the bioherm? Wilson (1975: p. 165-167) suggests formation through a combination of hydrologic accumulation and baffling by crinoids and bryozoa to form the muddy core facies. Gentle currents winnowed the sides of the bioherm.

Again, it is interesting to draw comparisons between these mounds and modern lithoherms in the Straights of Florida. The processes involved in their formation include (1) hydrologic accumulation, (2) biogenic sediment contribution and biologic entrapment of sediment, and (3) subsea lithification by marine cement. An organic framework, typical in modern reefs, is absent in these Mississippian mounds and is not a requirement for mound growth. Although organisms and hydrologic regime are not directly comparable between modern lithoherms and Muleshoe Bioherm, a combination of the three processes active in modern lithoherms could probably account for the features we see in these Mississippian buildups.

The diagenesis in non-biohermal Lake Valley sediments has been studied in considerable detail during the last 10 years. Using cathodoluminescent petrography, Meyers (1974) found five generations of cement in crinoidal grainstones like those we observe off the flanks of Muleshoe Bioherm. Most of this cement occurs as syntaxial overgrowths on crinoidal sands. Marine cements like those in the bioherm core facies are rare in interbioherm areas. The syntaxial, clear overgrowths are interpreted to have formed in a freshwater aquifer that occupied Lake Valley sediments during periods of sea level change and regional subaerial exposure. Careful examination of cements at post-Lake Valley unconformities revealed three cement zones to be preRancheria Formation and two more to be post-Rancheria but pre-Gobbler deposition. These two periods of subaerial exposure led to a great porosity loss in these sediments and resulted in $90-95 \%$ of the total intergranular cement in Lake Valley grainstones (Meyers, 1978).

From outcrop of core facies beneath the Muleshoe "arch," hike back downslope to vehicles.

OPTIONAL STOP. Retrace route east along the base of the bioherm escarpment about 150 yards to a prominent gully. Cross gully and scramble up dipslope, angling westward to top of bioherm. CAUTION! The route is considerably more steep and more difficult than that below. Use careful judgment in making this climb. Excellent exposures of steep flank beds are crossed during the ascent and the 
exposure gives one a real "feel" for the original depositional slope. On top there are well exposed examples of core mudstones, submarine cements and clastic dikes. The best exposures are above the cliffs in the southwest side of the bioherm. Cross the top of the bioherm to the north side for excellent view of the upper Lake Valley members lapping the sides of the bioherm and the unconformable relationships between the Lake Valley, Rancheria and Gobbler Formations. A descent can be made down the gully between the bioherm and surrounding strata of the north side. Contour around the base of the bioherm to the southwest side, and then descend the slope to Muleshoe Canyon and back to the vehicles.

Retrace route back to US 54 .

$\begin{array}{lll}4.8 & 16.7 & 80.2 \\ 5.2 & 21.9 & 75.0\end{array}$

Junction of ranch road and US 54. Turn left (south) on US 54.

Isolated buttes to the right are of varied origin. The closest is composed of Tertiary intrusives. Those beyond are outcrops of the Hueco and Yeso Formations. The San Andres Mts. are the prominent range in the background. This block-faulted range exposes a complete south-central New Mexico Paleozoic section from Precambrian basement on the east through Permian San Andres Formation on the west side of the range.

$9.5 \quad 31.4 \quad 65.5 \quad$ Stabilized reddish sand dunes in valley floor. Jarilla Mts. ahead on right expose Pennsylvanian and Permian sediments and Cretaceous and Tertiary intrusives. Southern end of the Sacramento Mts. and the Otero Mesa are in the distance to the 1eft. The Otero Mesa is composed of the Yeso and Hueco Fms.

$\begin{array}{rrr}14.5 & 45.9 & 51.0 \\ 0.2 & 46.1 & 50.8 \\ 4.3 & 50.4 & 46.5\end{array}$

Orogrande town limit.

Orogrande Post office.

Directly west are the Organ Mts., a southern extension of the San Andres Mts. They expose large areas of Cretaceous and Tertiary volcanics and some Precambrian intrusives and Paleozoic sediments.

$18.0 \quad 68.4 \quad 28.5 \quad$ North end of Franklin Mts. at about 1 o'clock.

To the right are the Hueco Mts. in the distance. The bulk of the Hueco Mts. is Pennsylvanian and Permian, but the highest peaks are Cretaceous and Tertiary intrusives.

$\begin{array}{lll}7.7 & 76.1 & 20.8\end{array}$

Texas state line and El Paso city limit. Note: we are really quite a way from $E 1$ Paso at this point.

$8.7 \quad 84.8 \quad 12.1$

Junction with Texas 2529. Take $45^{\circ}$ left turn onto McCombs Street for airport or Caballero Motel. Continue straight ahead for downtown E1 Paso. This route goes to the airport.

$\begin{array}{rrr}2.0 & 86.3 & 10.1 \\ 3.1 & 89.9 & 7.0 \\ 3.3 & 93.2 & 3.7 \\ 1.8 & 95.0 & 1.9 \\ 1.9 & 96.9 & 0.0\end{array}$

Bear right onto Railroad Drive.

Take overpass (Marshall Road).

Take left on Fred Wilson Road.

Fred Wilson Road makes a sharp right.

Left turn to entrance of E1 Paso airport. 
Figure 3 . View from Fresnal Box Canyon Vista showing downdrag on Bug Scuffle Limestone Member of the Gobbler Formation on the eastern side of the Fresnal Fault.

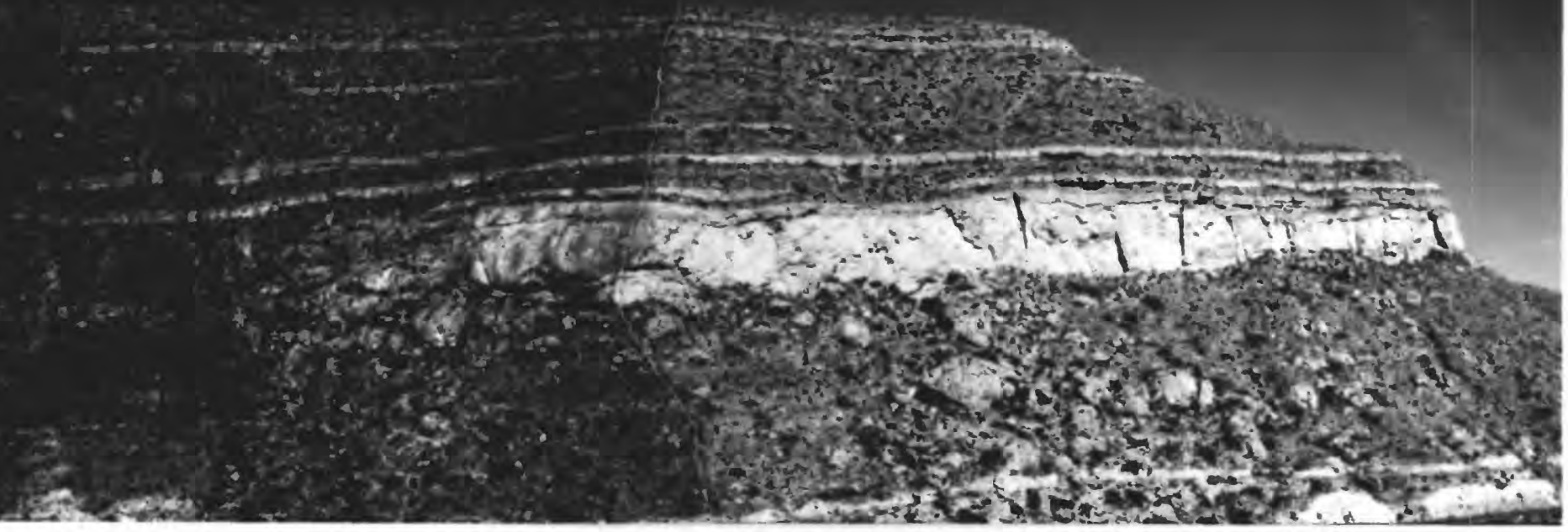

Figure 4. View of Virgil Bioherm from south side of Dry Canyon. Note steeply dipping flank beds in massive, cliff-forming limestones. Beds which cap the bioherm drape over the west end of the exposure.

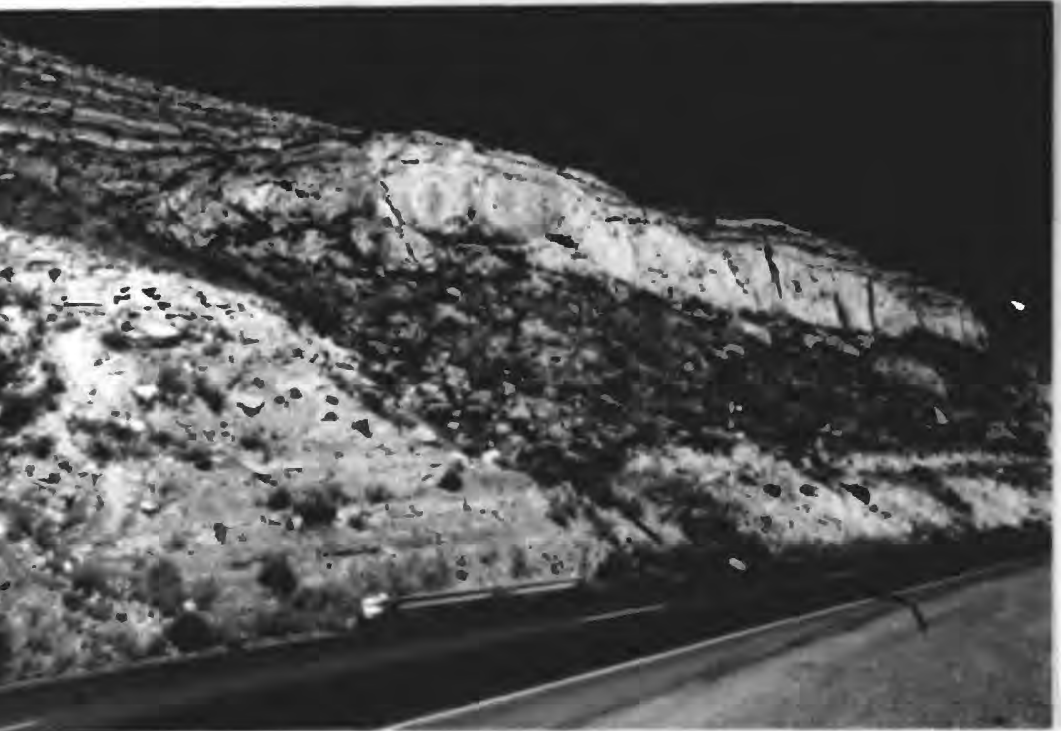

Figure 5. Field trip route (dashed) for STOP IV-1, around west end of Virgil Bioherm. 


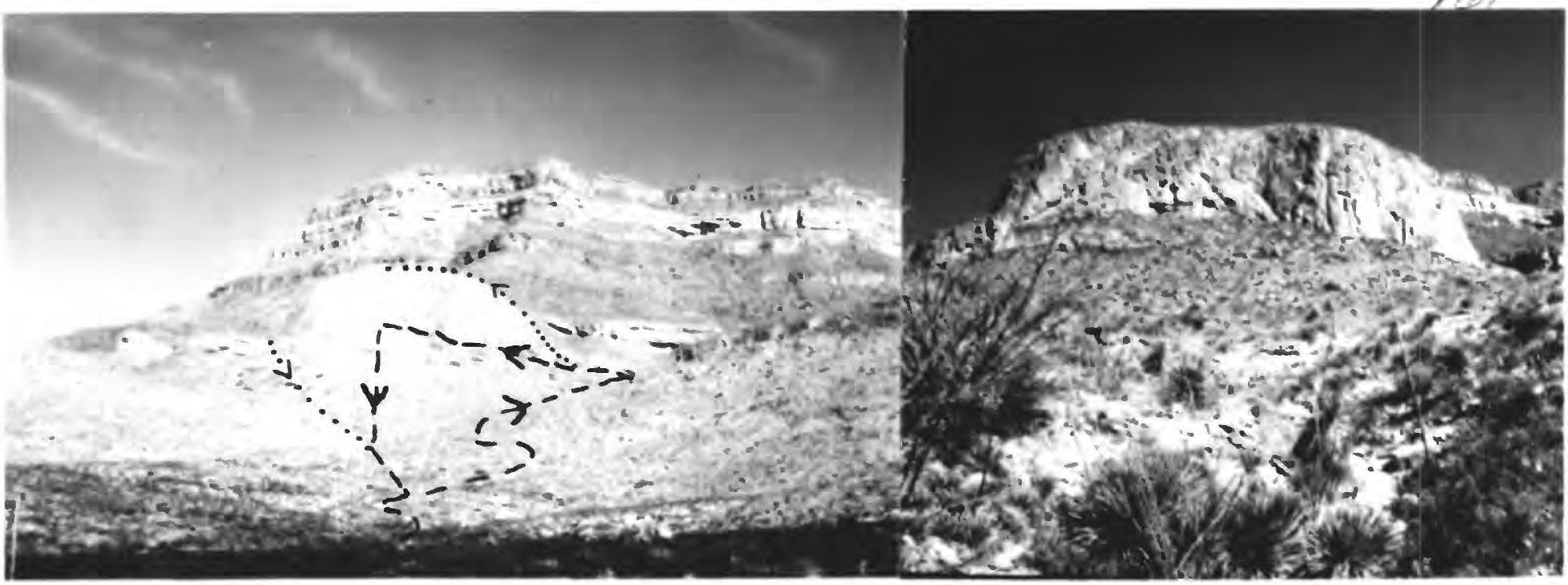

Figure 9. Left: field trip route for STOP V-1 (dashed, see roadlog for details) and optional route (dotted). Right: detail of Muleshoe Bioherm illustrating arched and dipping flank beds exposed in southwestern escarpment. Core facies is exposed beneath center of arch.
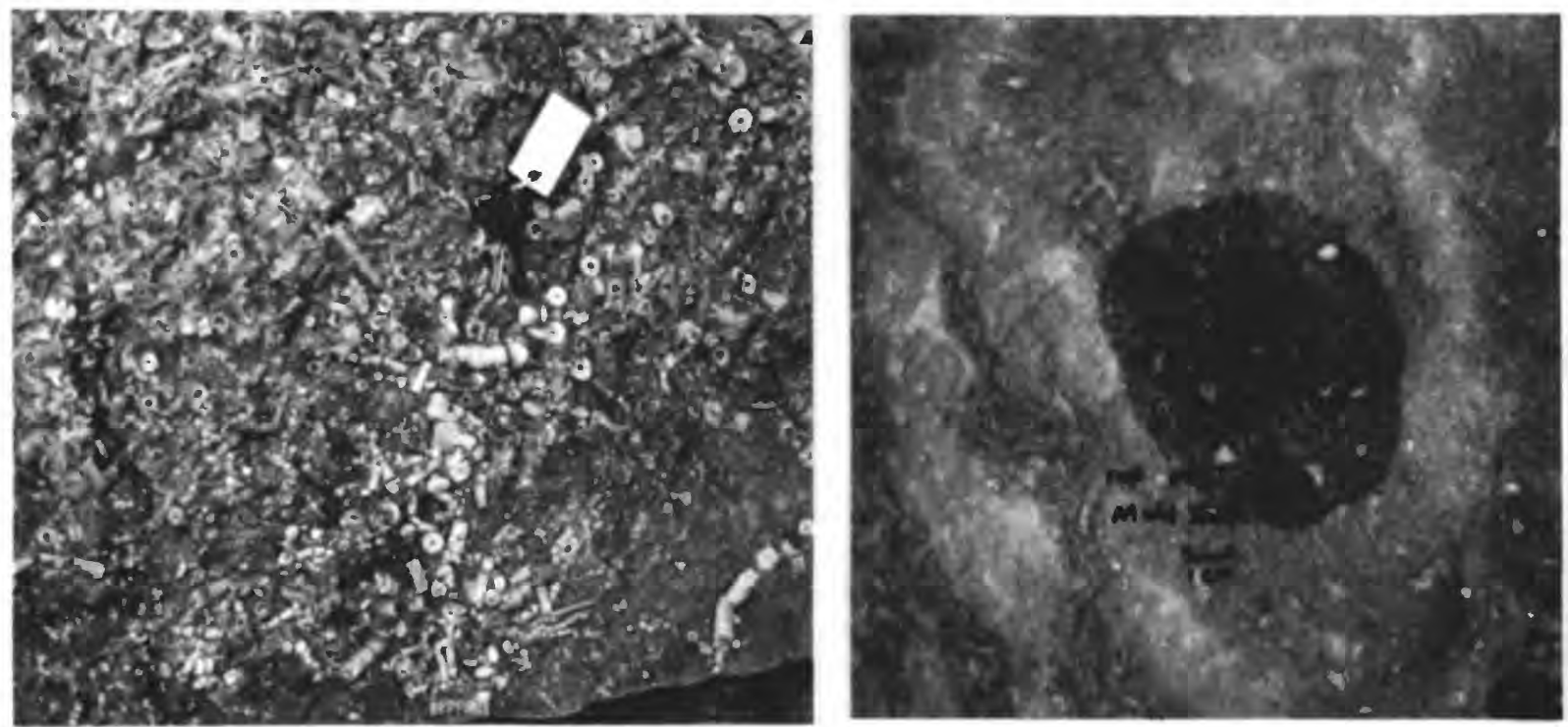

Figure 10. Left: typical outcrop (bedding plane) of Muleshoe crinoidal grainstone illustrating poor sorting and articulated sections of crinoid columinals. Right: Labyrinthine appearance of bryozoan fragments coated with marine cement. Outcrop of core facies on top of bioherm.

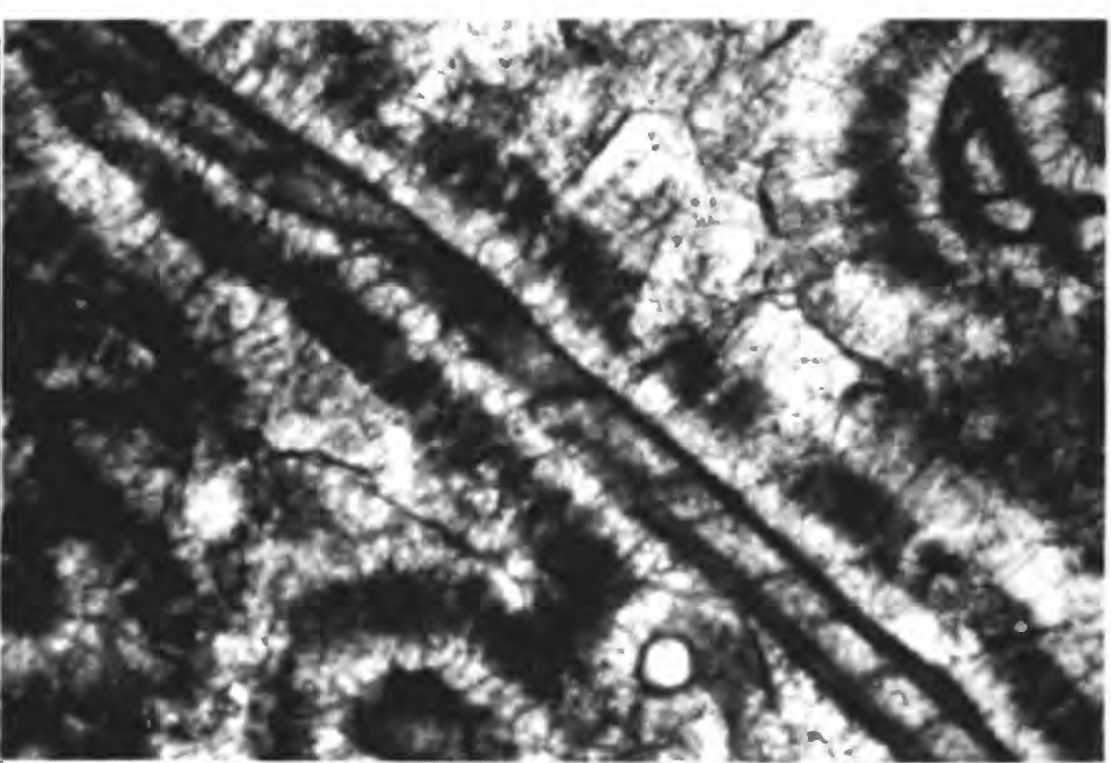

Figure 11. Photomicrograph of bryozoan fragments coated with banded, cloudy cement. This cement is interpreted as syndepositional, marine cement. Field of view is $3 \mathrm{~mm}$. 

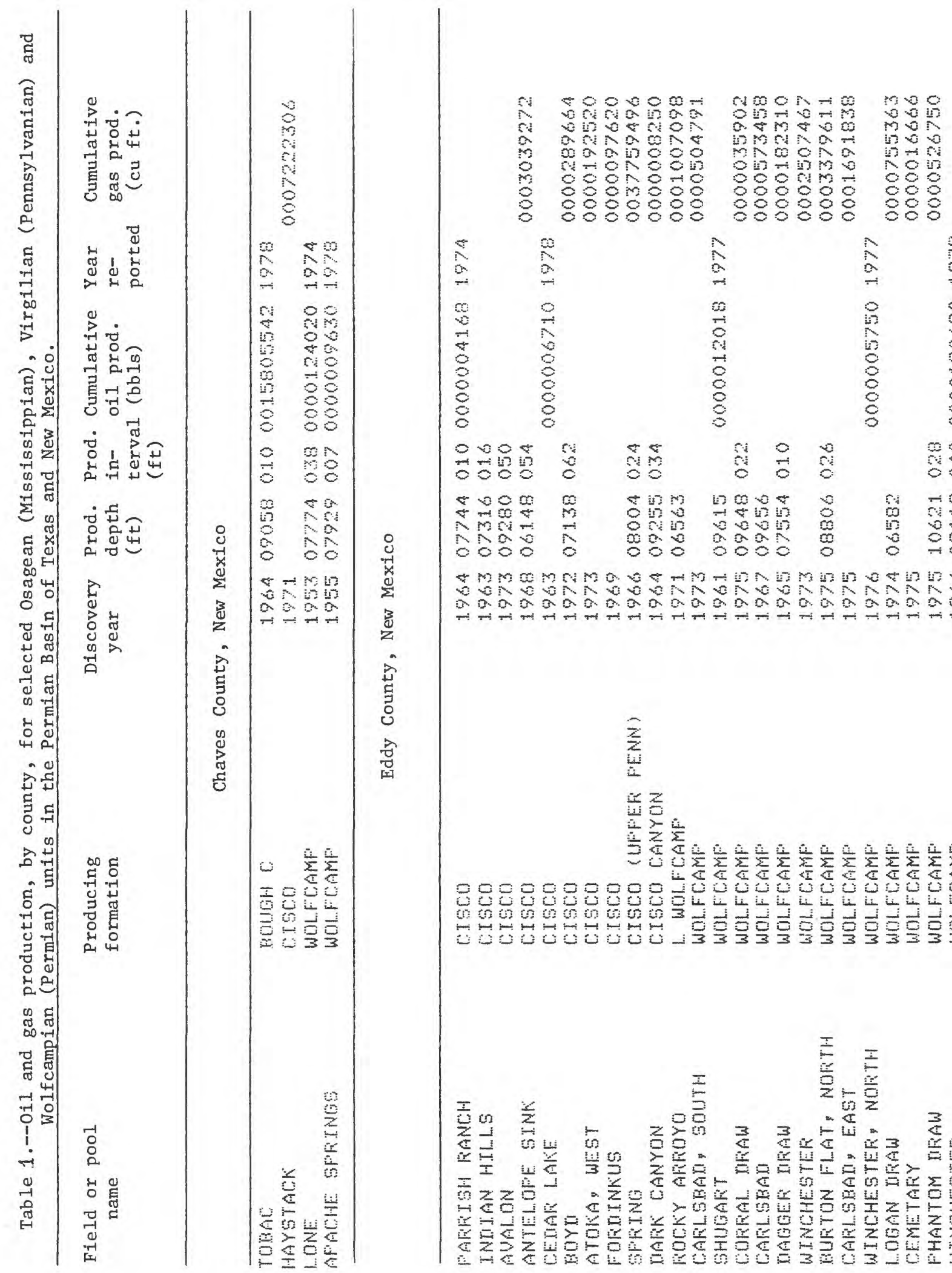

$\begin{array}{ll}1 & 0 \\ 2 & 0 \\ 1 & 0 \\ 0 & 0 \\ 8 & 0 \\ 8 & 8 \\ 8 & 8\end{array}$

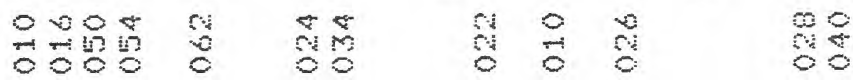

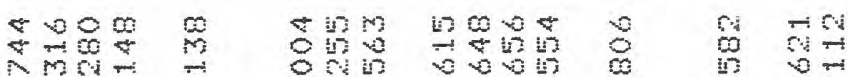
रिवे

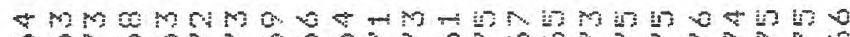

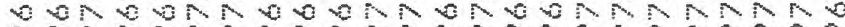
a. 0000000000000000000000 8

8

8

8

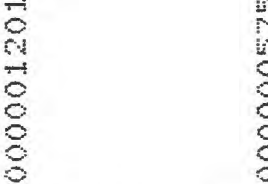

$\frac{5}{5}$

픈

$+2$

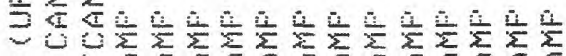

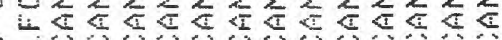

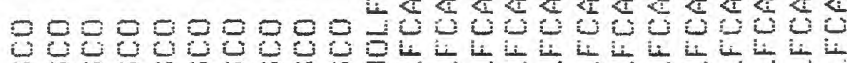

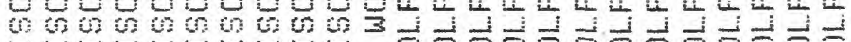

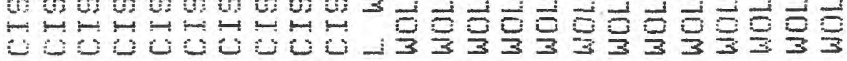

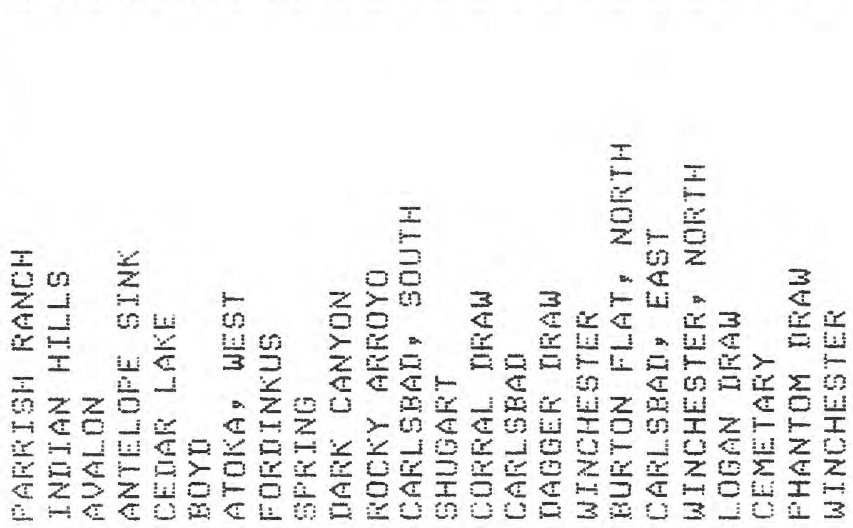




\section{18
28
88
88}

08060

वृos? उषिम 000 780050 8888880

$\stackrel{\infty}{2}$

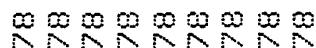

OSONOSOS

20202020

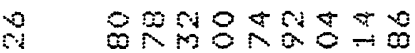

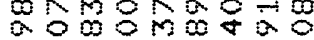

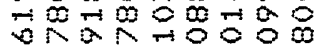

$80 \% 80 \% 0$

888880880

888888880

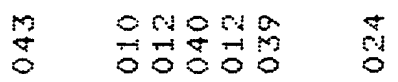

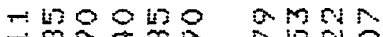

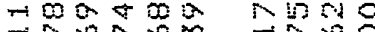 \\ Tron

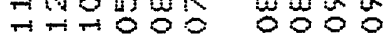

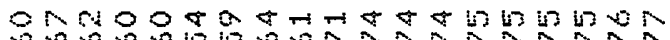
$2000041700 N N A N A N A N$

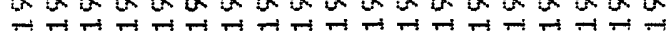

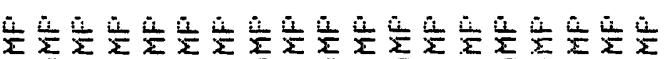
द

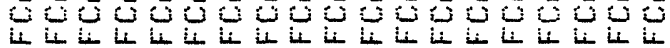

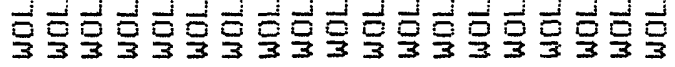

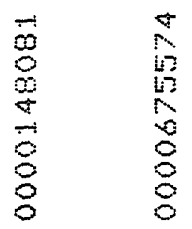

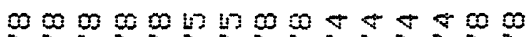
NNNNNSNNNN N

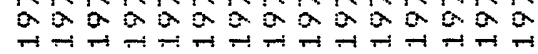

$\infty \infty \infty$ $\lim _{\rightarrow \rightarrow \infty}$

$\sin$

क

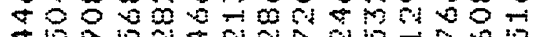
स W mNmon+mon

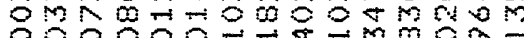

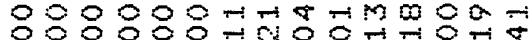

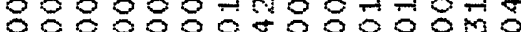

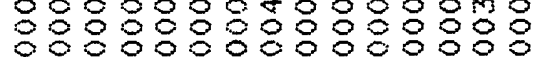

- $\rightarrow+\div$

$0+\infty+\infty \quad 000$

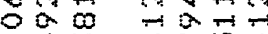
रषफ a $54=$

$\rightarrow 20080$

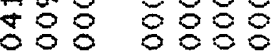

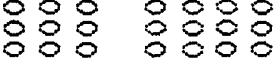

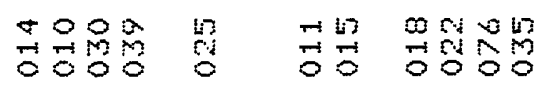

$\underset{0}{0}$

-10000000 mo

mor

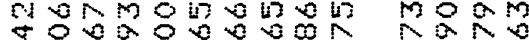

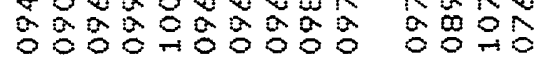

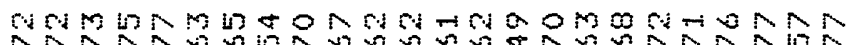

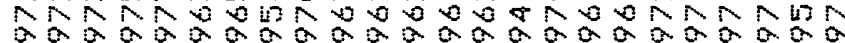

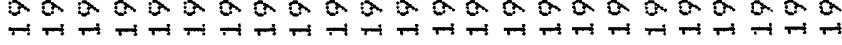

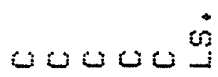

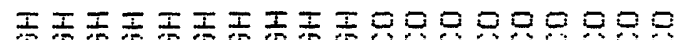

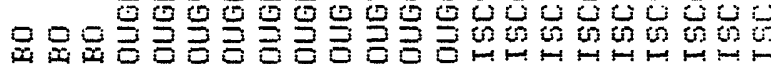

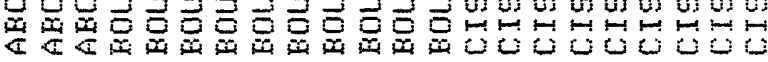

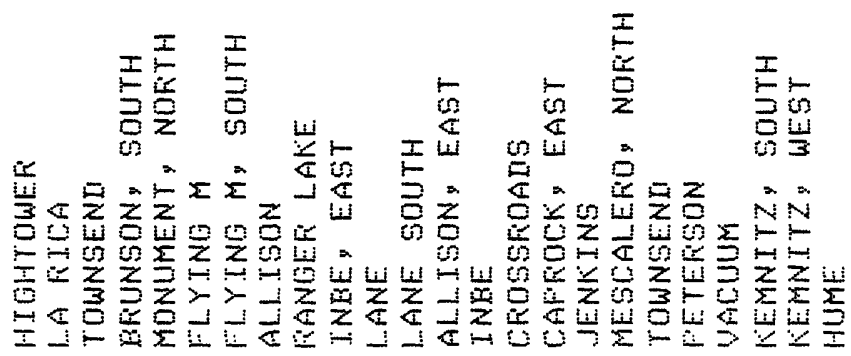




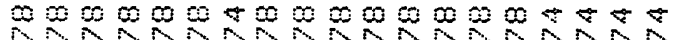

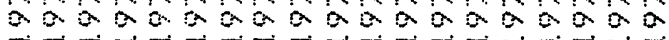
애유

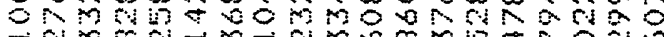

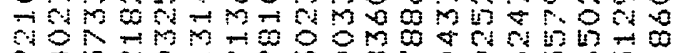
कै

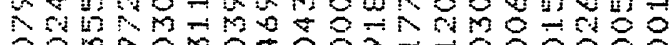

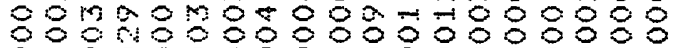

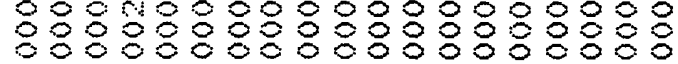
m:

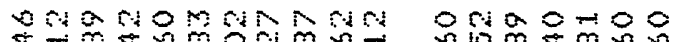
\& $\rightarrow+000000000$ $040 \% 000$ 0200000

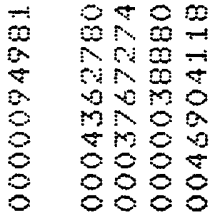

$\infty$

2

s

So

m

8

8

$+8$

$\infty$

62

in
क巾

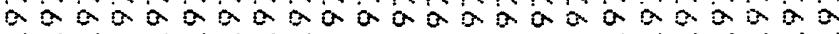

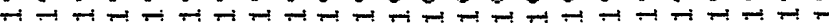

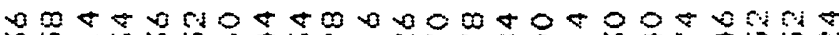
MHMm -50\%0

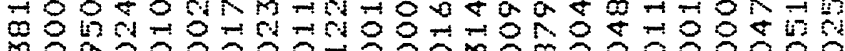

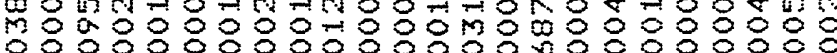

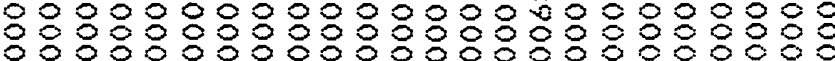

\section{Ninton}

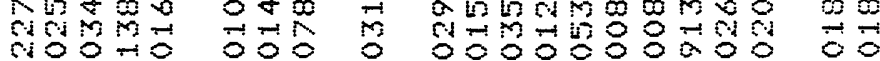
wo

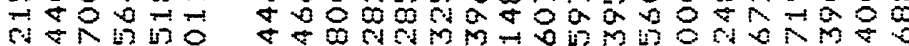

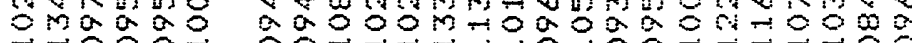

A.

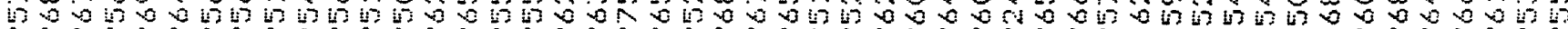

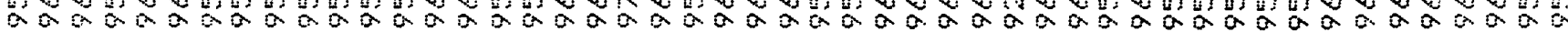

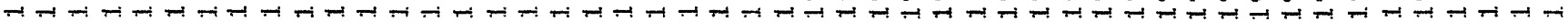

$\frac{1}{6}$

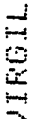

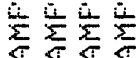

$z \underline{a}=$

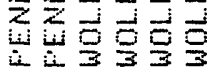

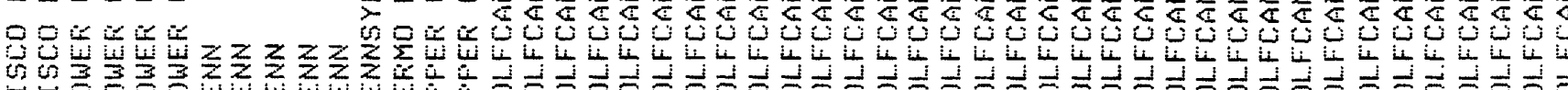

\section{$\geq \geq \frac{z}{2} \geq \geq$}

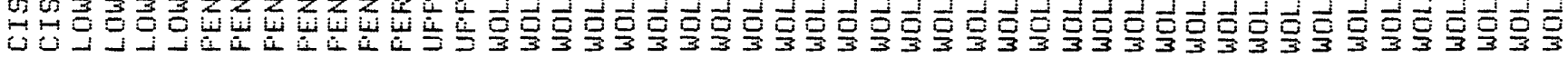

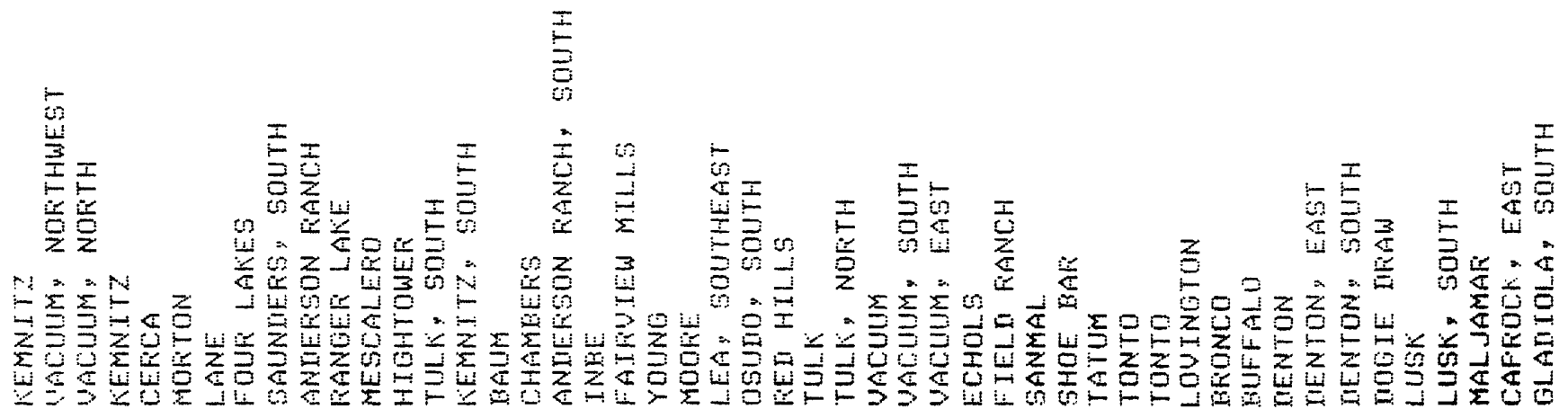


$\square$
$\stackrel{1}{0}$
0
0
8
8
0

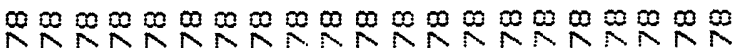

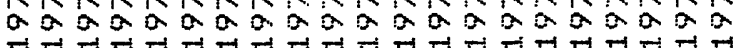
$000000000+2000000 \%$

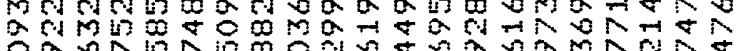

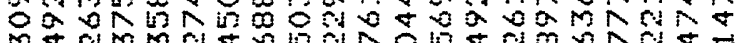
Mष

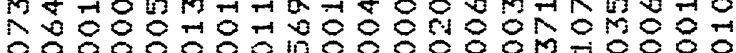

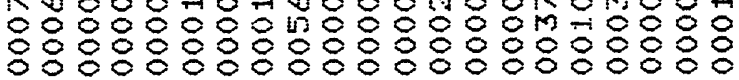

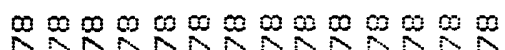

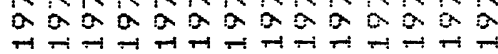

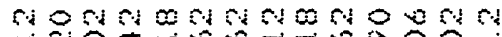

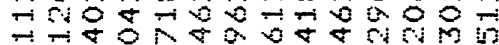
mom

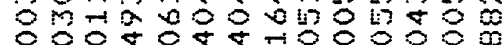

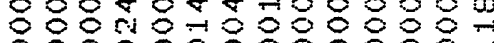

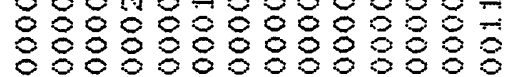
사유

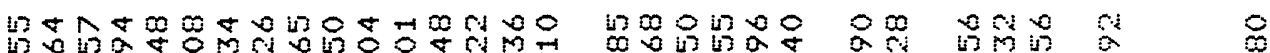

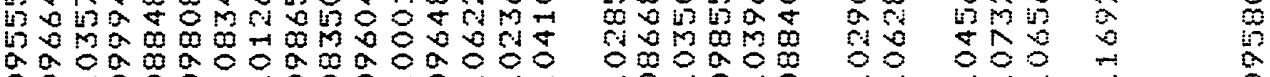

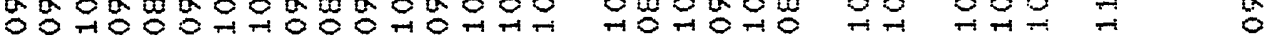

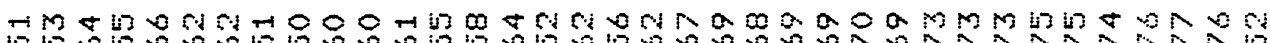

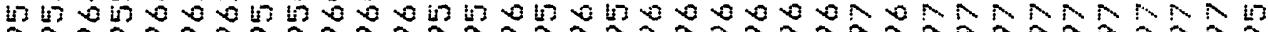

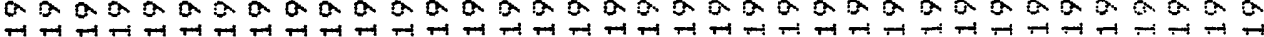

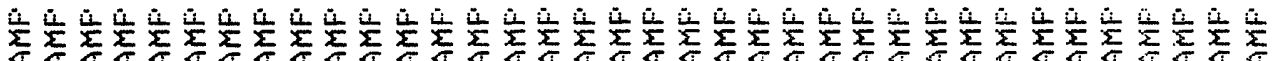

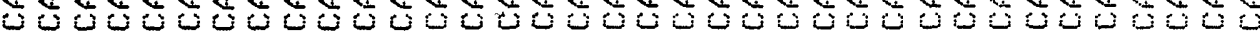

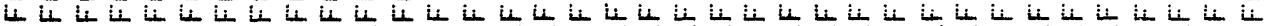

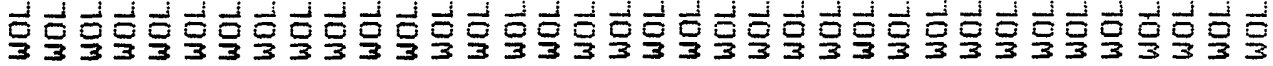

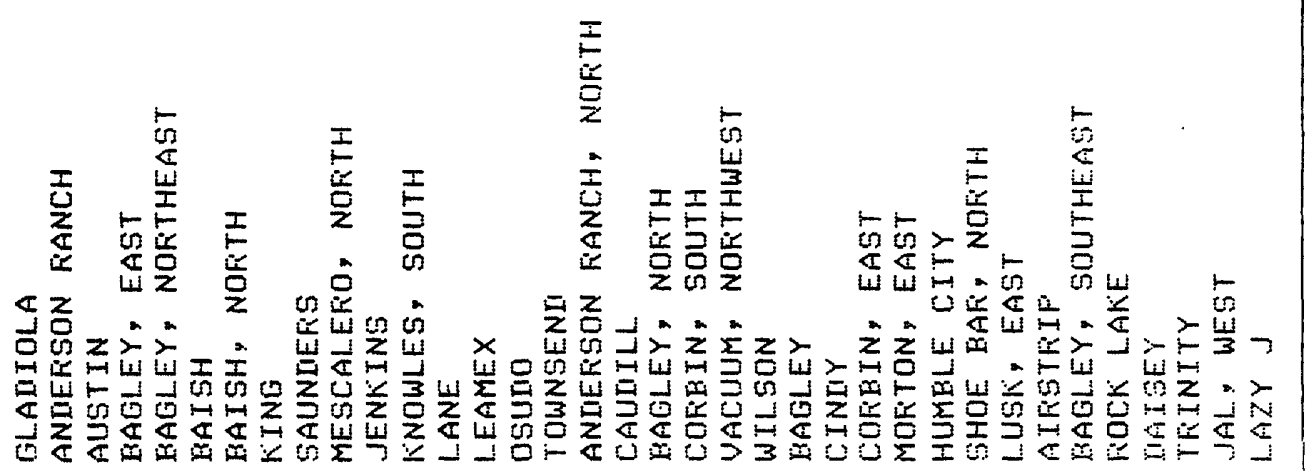

$\begin{array}{ll}5 & 0 \\ 0 & 0 \\ 0 & 0 \\ 0 & 0\end{array}$

$\operatorname{Rin} \infty$

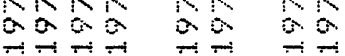

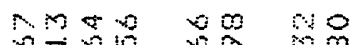
$0=0 \mathrm{~m}$ कर

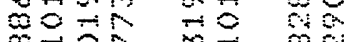
क야 M

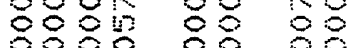

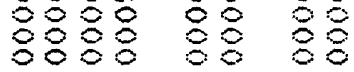
苟 $m \infty 0 m \infty 0 \%$ $200 \mathrm{jog} 0$ तN

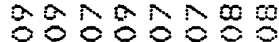

मान

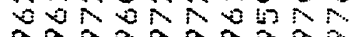
2020200002 $\rightarrow \Pi \rightarrow+T+T-$

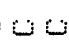
$\frac{5}{i} \frac{i}{i}$

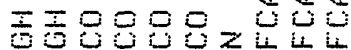

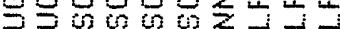

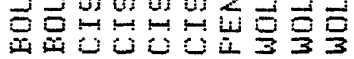

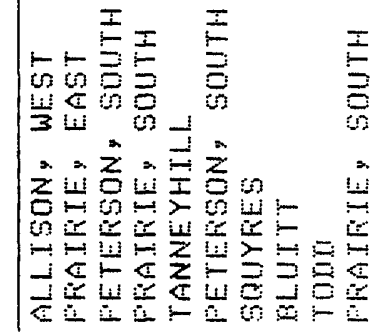




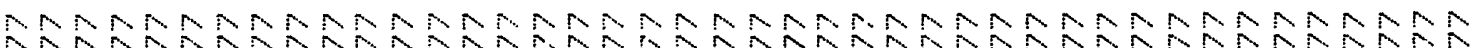

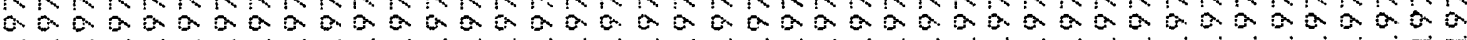

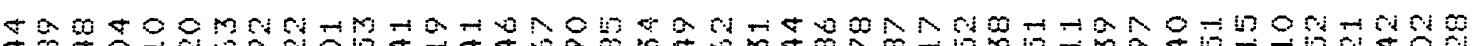
40\% an

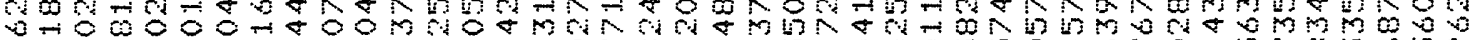
क्.

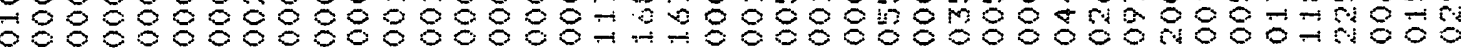

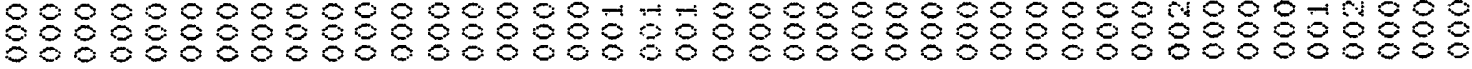

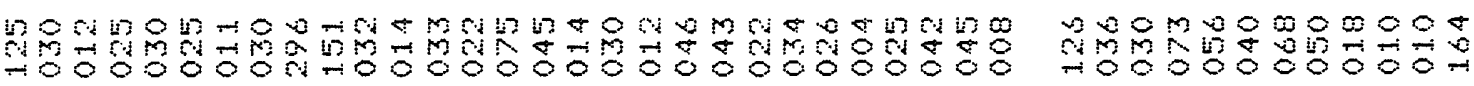

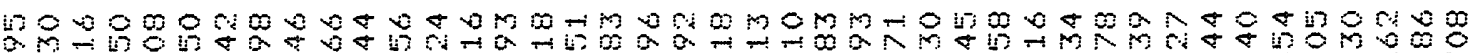
m-20mb

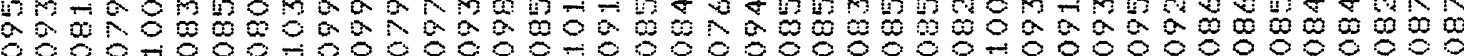

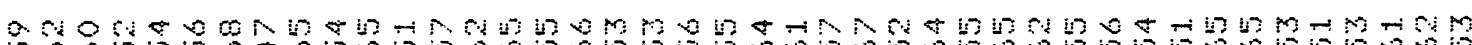

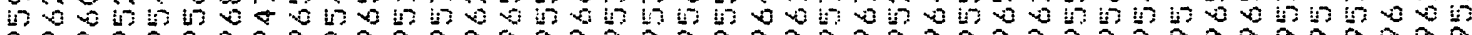

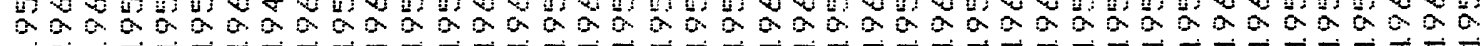

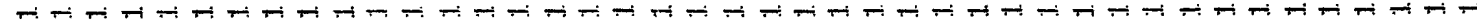

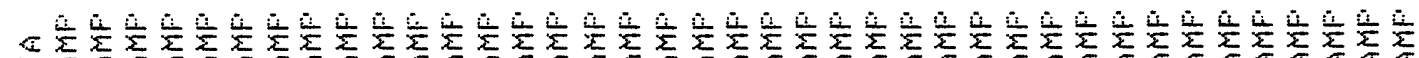

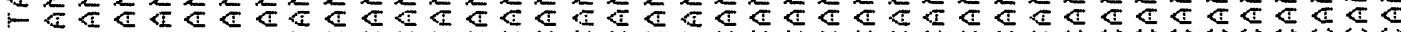
zz

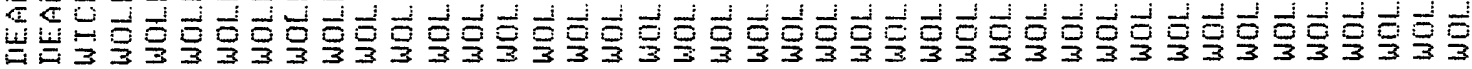

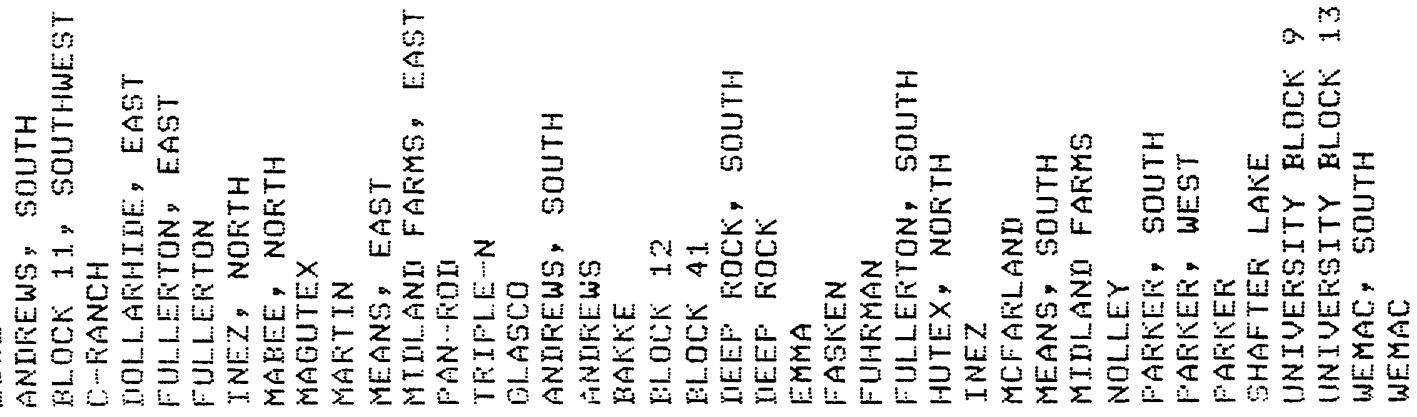




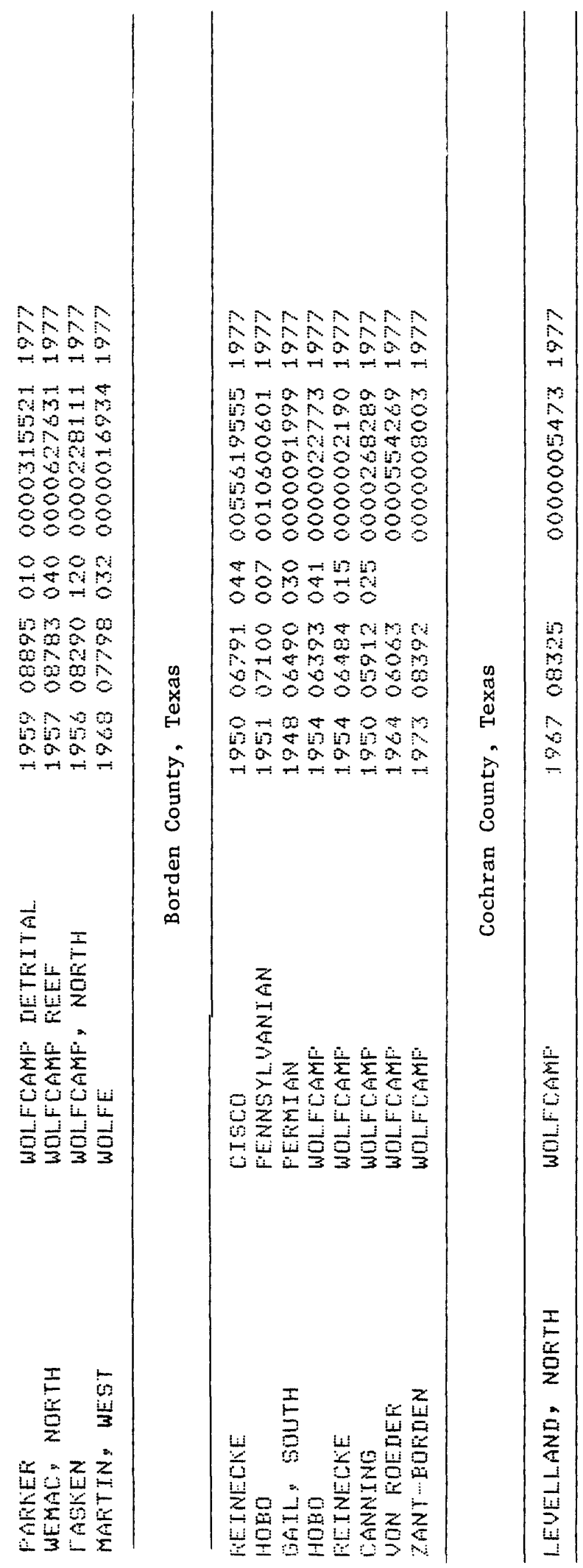

NARNARR $\infty_{\rightarrow \infty \alpha 2020}^{20}$ 00 : $\infty N \infty \leftarrow \infty$

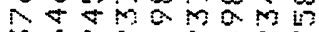
$200 a-a+c h$ $0 m+\pi m$

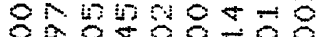
$088 \% 8808$

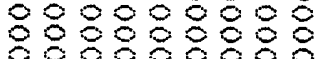
in miston $80088808 \%$ क 0 n

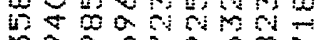
$\rightarrow \infty \infty \infty a b \infty$ वे 120 mom-1\%

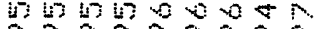

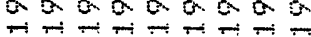

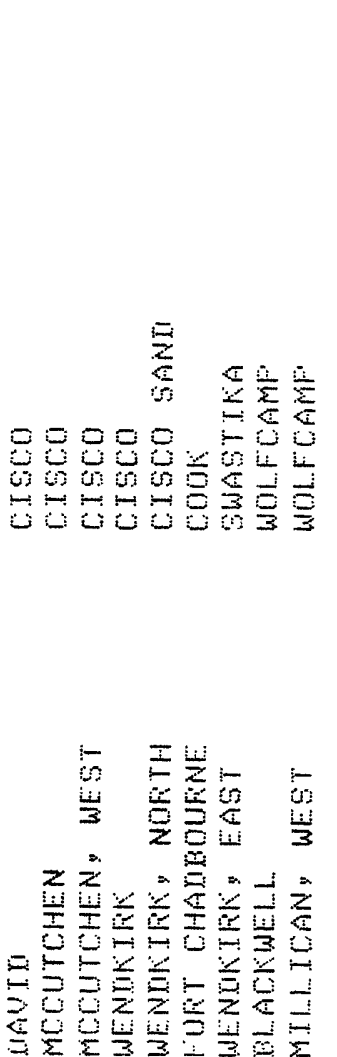

0
0
0
0
8
0

WNONNDN 200 abo200

wo $0 m+20 \%$

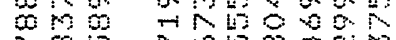
का 50 bo

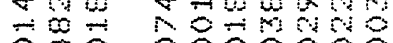

\%०

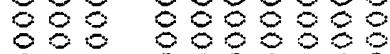

ㅇㅇ 8880888

며 6영영

1)

mi2 osoptom

की रकी०ष०की

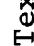

बकाम की $2002020 \operatorname{lng} 200$

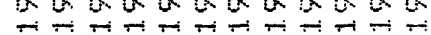

Lij

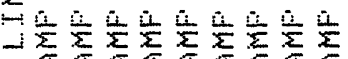
$=0000000000$

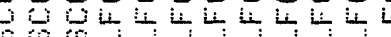

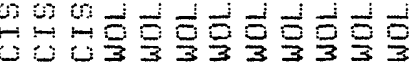

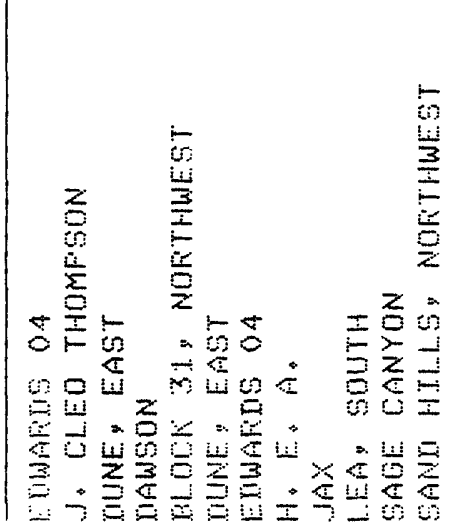


3
3
0
0
0
0
0

प) पNANA करू०ूर $\rightarrow$ بा بा 8 isonomo in rito ómorma

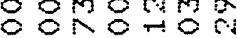

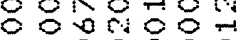

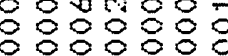
8888808 is $000 \mathrm{mon} 0$ +응ㅇㅇㅇ영 0 in 0012000 人in

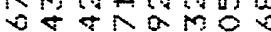
iम $0 \rightarrow \infty N N \infty \infty$

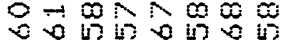
004020015 $\rightarrow \rightarrow \rightarrow H \rightarrow H$

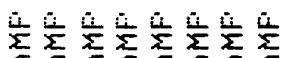

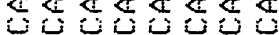
iL 4 is

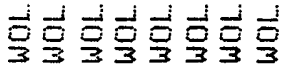

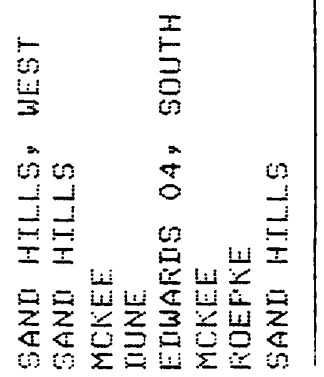

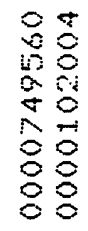

NANA NNANANN

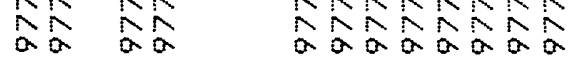

$\begin{array}{ll}0 & 2 \\ 4 & 8 \\ 0 & 0 \\ 0 & 10 \\ 0 & 2 \\ 0 & 8 \\ 0 & 8 \\ 0 & 0\end{array}$

10
2
0
0
0
0
0
0

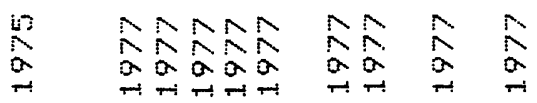

in an anonom

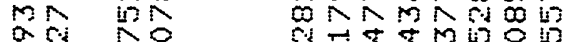

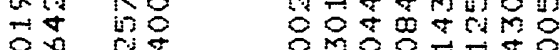

80 व.

88 8용

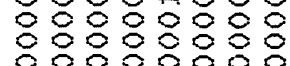

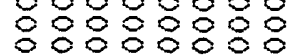

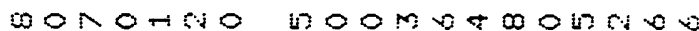

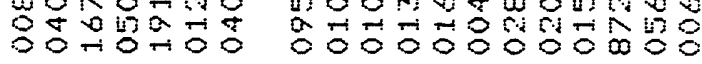

0000

$m \infty \begin{array}{ccc}\infty & 0 \\ 0 & 0 & 0\end{array}$

ب

क्रिएक

कom

in

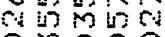

8080

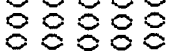

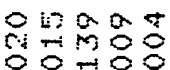

20

今q

$8 \mathrm{~m}$

-

两 8 क

ㅇㅇㅇㅇㅇㅇ

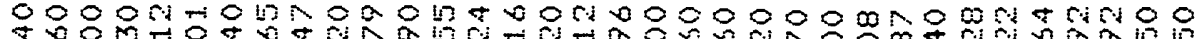

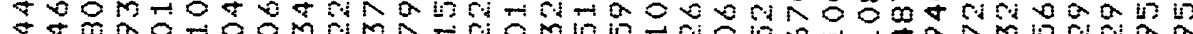
की 000000000000000000000000000000000

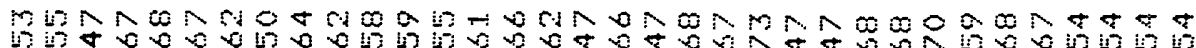

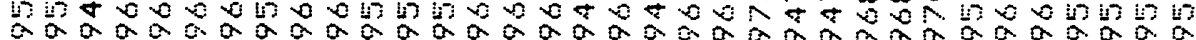

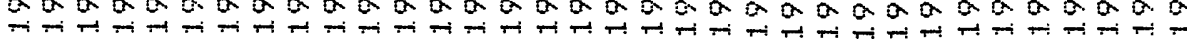

\section{$\stackrel{i}{\stackrel{i}{i}}$}

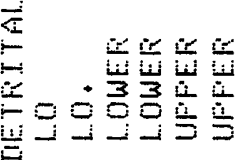

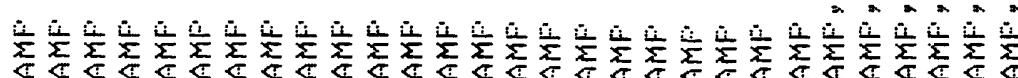
о 8980

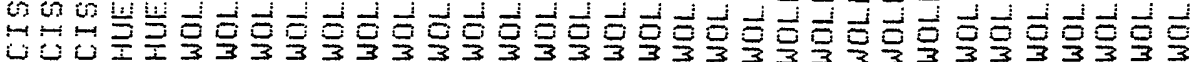

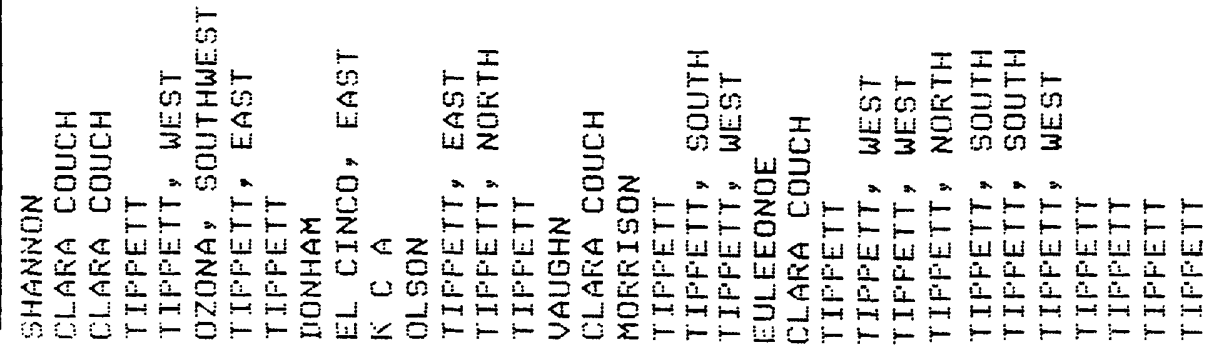


NNANNNANANNNN

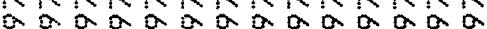

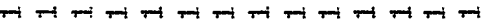
ON \& $10000 \% 0 \mathrm{~m} 0 \div \alpha$

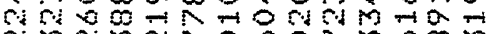
rmmmRoboNmbom a $050.588 \% 800060$

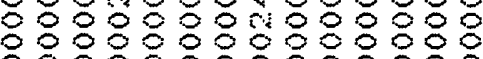
m whino on o क巾 00200000000 क600600060000

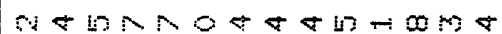
Notaby a 000000000000

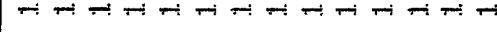

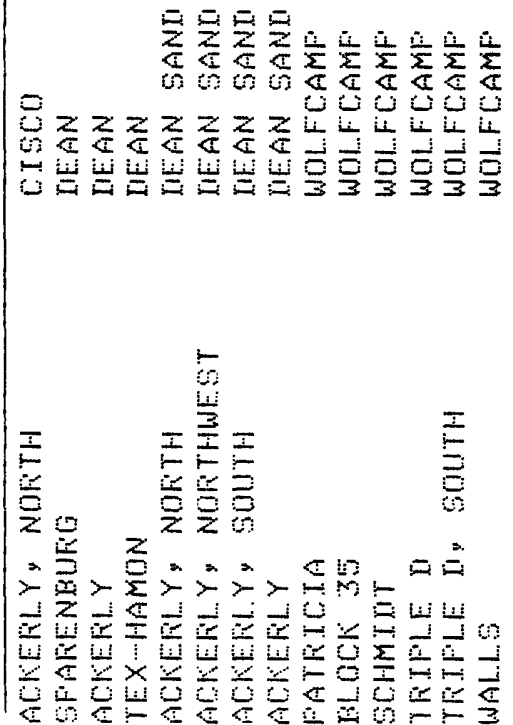

$\operatorname{SNRN}$ OANAR $a$
$\rightarrow \pi+\pi$ $\infty+1+\infty a t$ तक $m=\infty N \infty$ $M N F \&$ तथ $000 \mathrm{~m}$ 080800 8.8888

$\begin{array}{llll}0 & 3 & 4 \\ 0 & 0 & 8 & 0\end{array}$ $\operatorname{Rin} \theta$

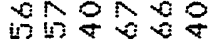

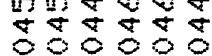
a m mom in 0000 202020

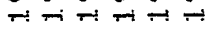

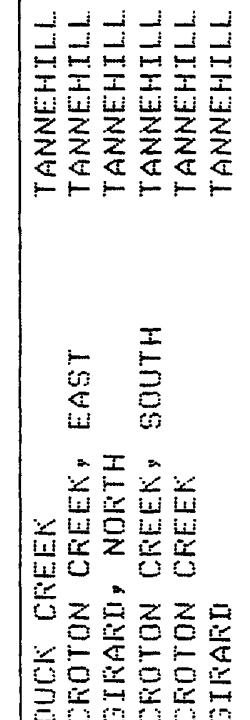

NANANANANANAN NNNNNANRNRNRNR

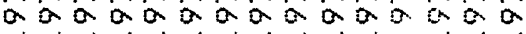
$m+\infty O H+M O M N N-M \infty \infty$ morid $000 \%$ in 01400

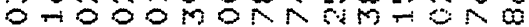

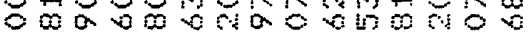
수의 084000008008000 8:8:88:808:88:88:80 WHRHMNOROF000

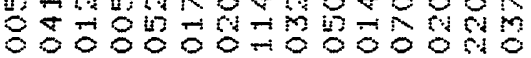

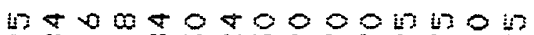
a क क0

wh baw

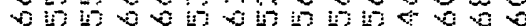

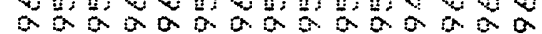

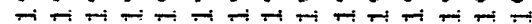

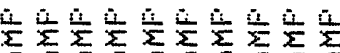

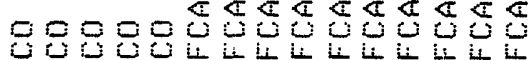
06

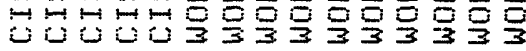

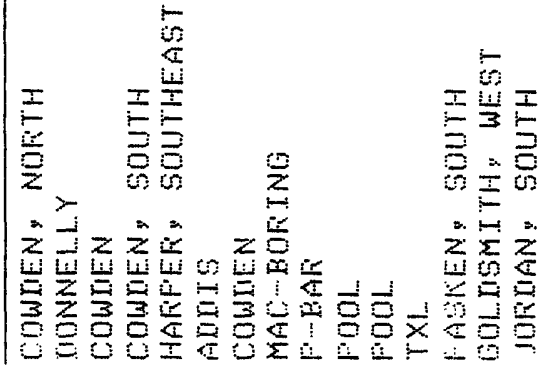


NNSK $20202 \Omega$ $\rightarrow \rightarrow \rightarrow \rightarrow \rightarrow-$

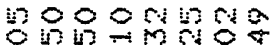
$R \rightarrow \infty N 20$ i से $17 \% 06 \mathrm{~m}$ तिना० कmino

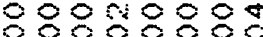
85868\%8\%

w to00内人

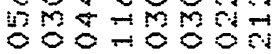

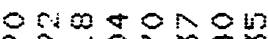

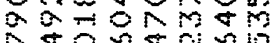
Dto

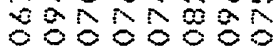

otatara Q 02002002

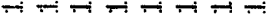

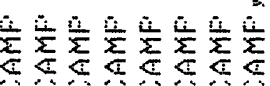

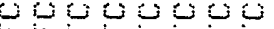
$4-4-4$ 比

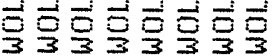

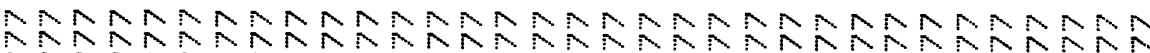

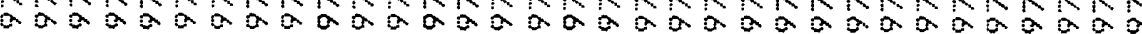

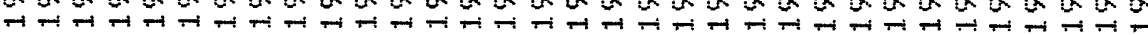

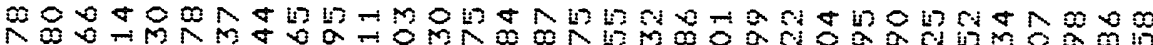

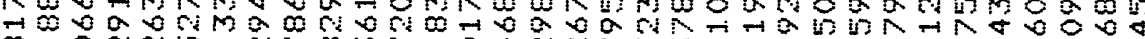

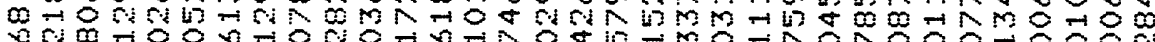

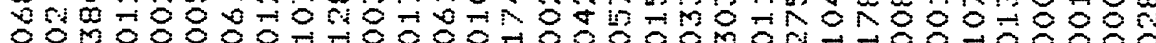

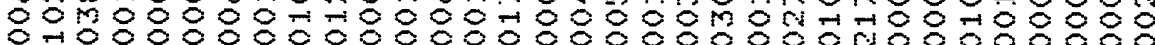

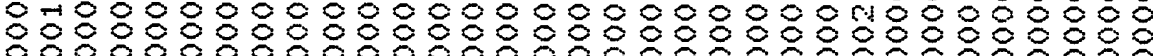

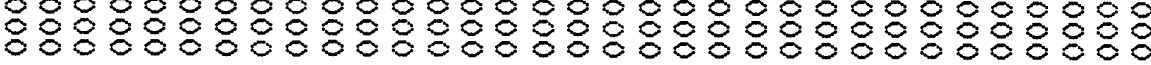
i

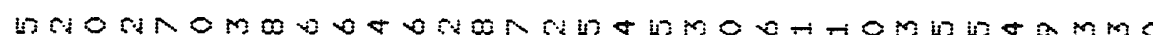
of mpton की m00

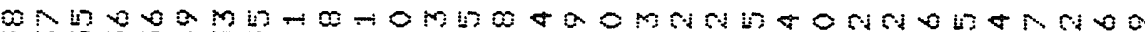

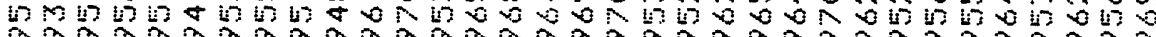
002020202020020202020020202020200

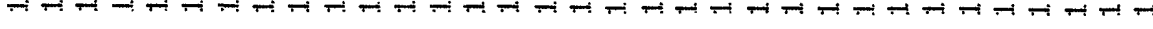

을

i

咅吉 岁是

娄

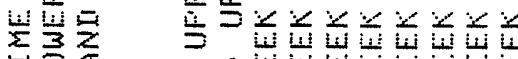

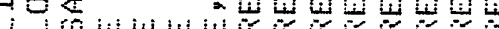

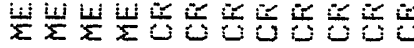

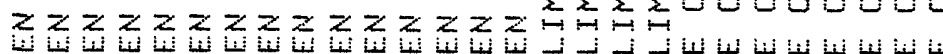

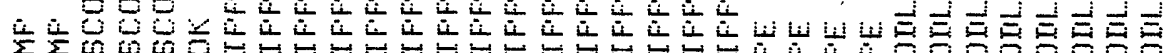

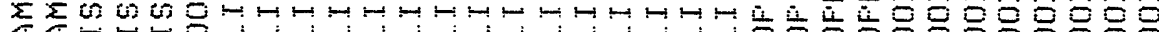

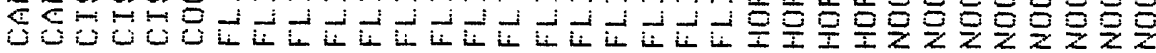

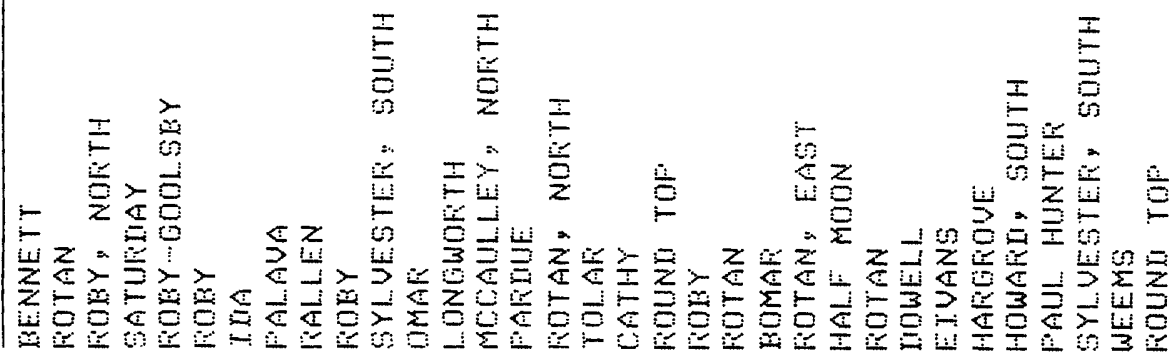




$$
\begin{aligned}
& 0 \\
& 4 \\
& 0 \\
& 8 \\
& 8 \\
& 8 \\
& 8
\end{aligned}
$$

NRA RPRARR

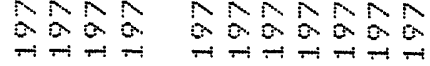

NANANANNANA

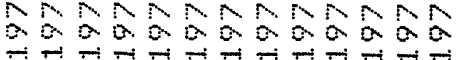
क० त8 50000000 00 6098 $888 \%$

8807588 8888888 8888888

$20+\infty 00,0100$ or

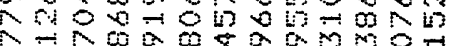

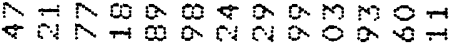

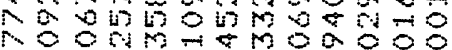

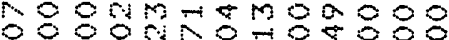

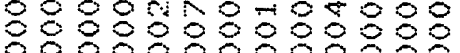

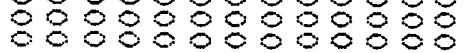

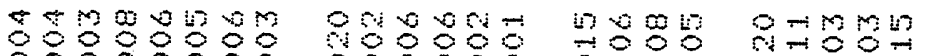

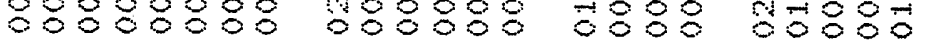
\%1 का मकी m.

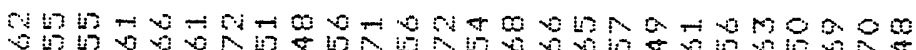

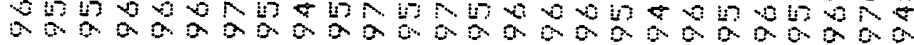

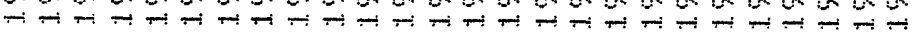

$\therefore \%$

in

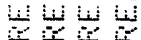

$\because \div$

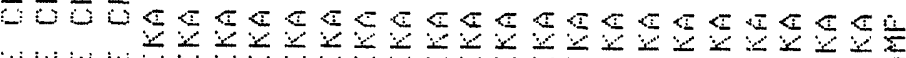
$4-1-10$

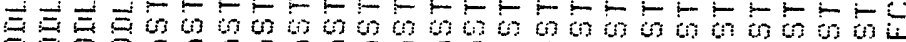

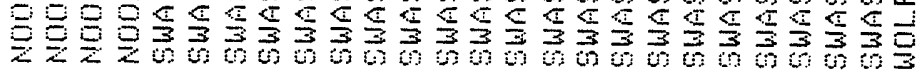

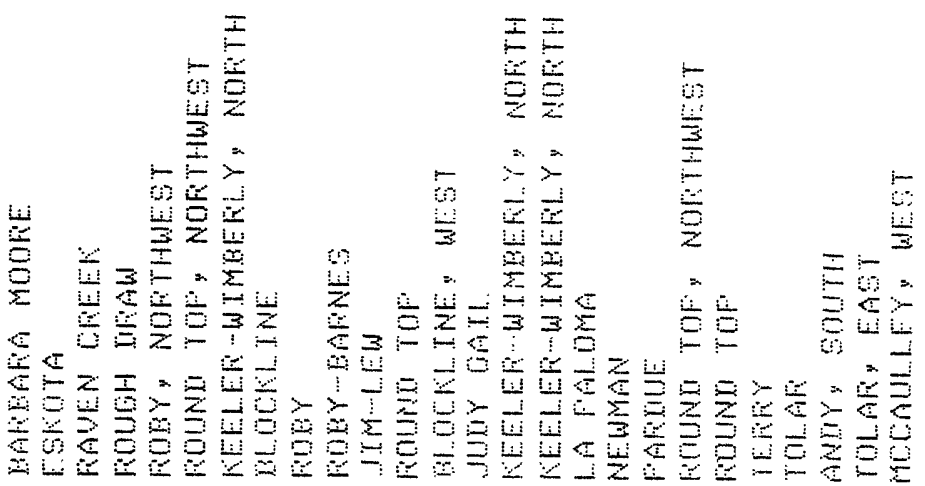

ANANANANANANANAN 20002020200200200000

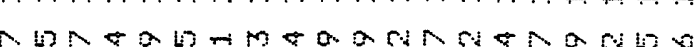

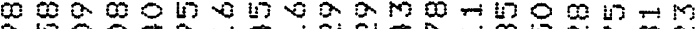
कWo

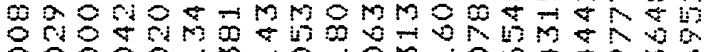
$80880 \div 0 \% 078 m-004 \% 2 m$

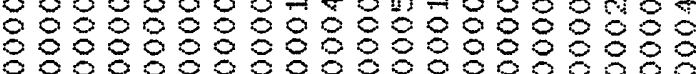
$\rightarrow \ln 12$

잉유

\&

$0050 \mathrm{0}$

WWNMETNW00090000\%80 -

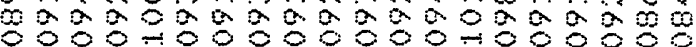

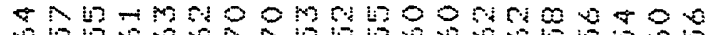
ond 20020200200000000000

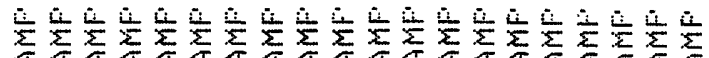

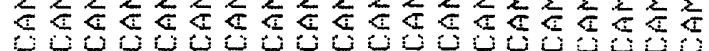

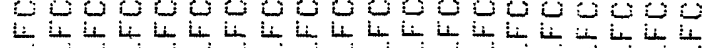

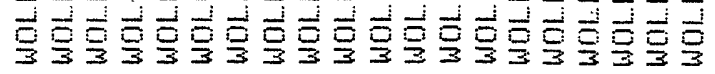

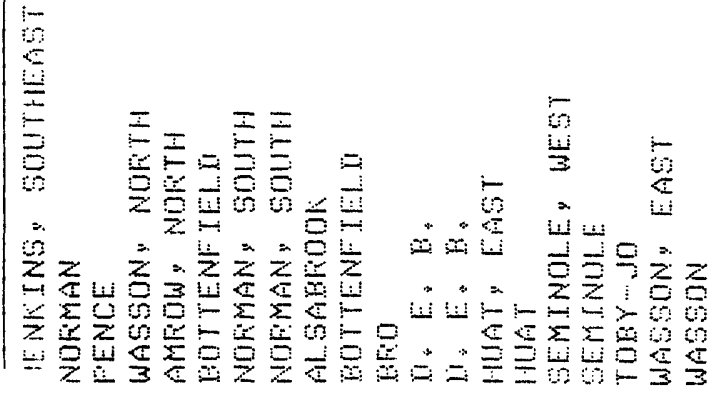


NRRRRRR 202020202 ०$20 m \mathrm{p}$ कीam $\mathrm{m}$ ती \& is $002 \div 0=$ - $0000 \%$

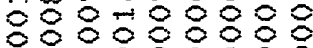

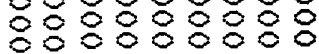

880

and in on 0 $0010+0200$ 200000000 - $4 \mathrm{man} a \mathrm{arat}$

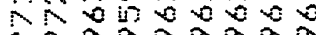
a 2020200

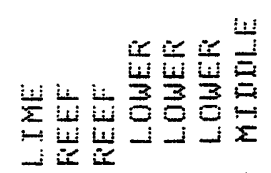

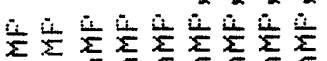

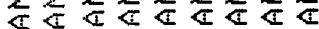

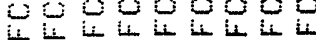

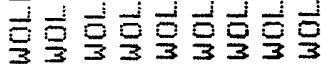

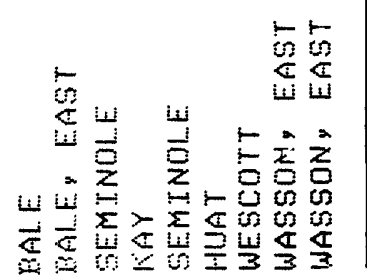

NANANANAN

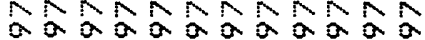
हरे $\lim _{\rightarrow \rightarrow \infty}$

ona

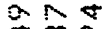
$+\infty$ 평 680 888 888

8

mas



$\leftarrow 0$

100

$\mathrm{N}=$

$\operatorname{lin}_{0} \infty 2$

$\rightarrow \rightarrow+$

on ompoanton 재에

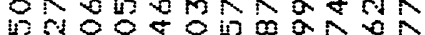

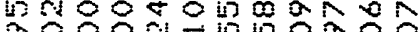

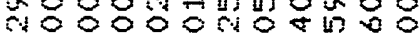
o0\%0000 00000

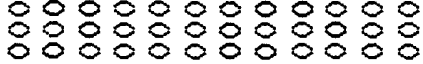
영 Fक山ष otrot a is ot 000

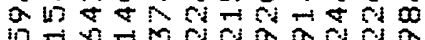

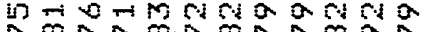
जकी स\&MR $200 \mathrm{an} 200 \hat{2}$

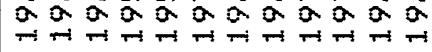

$\frac{5}{x} \frac{1}{x}$ 近

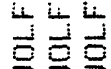
弹

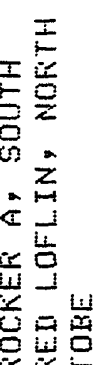

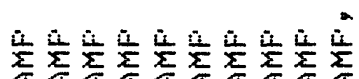

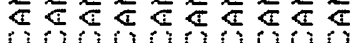

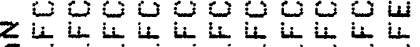

is

I记

5

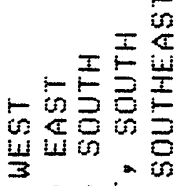

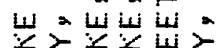

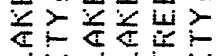

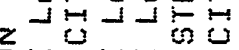
$z^{2 x} z$

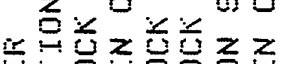

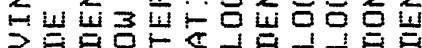

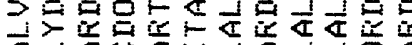

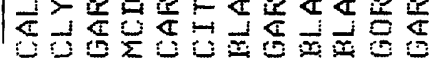

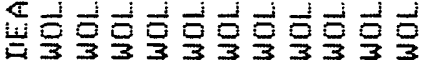

ANRAANARANANRN ลNANANANANA⿵人

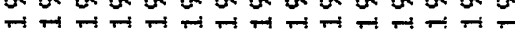

Om

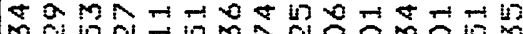
MWNFM m

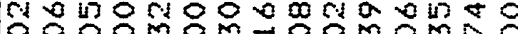
OOOMOMHOOMOMNO

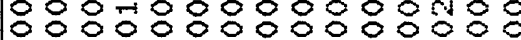

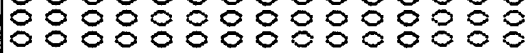

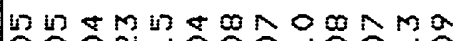

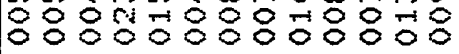

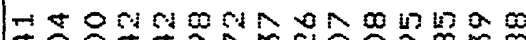
क०O क

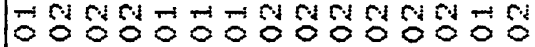

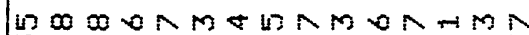

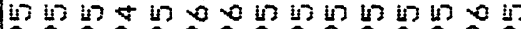

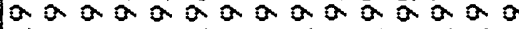

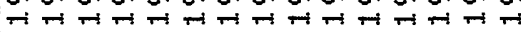

$\frac{1}{3}$

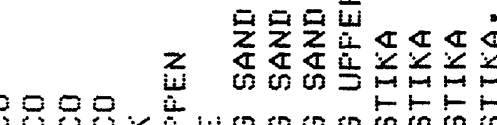

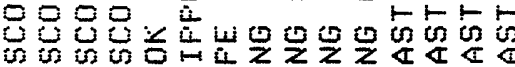

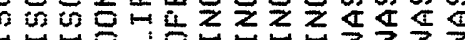

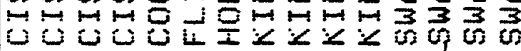

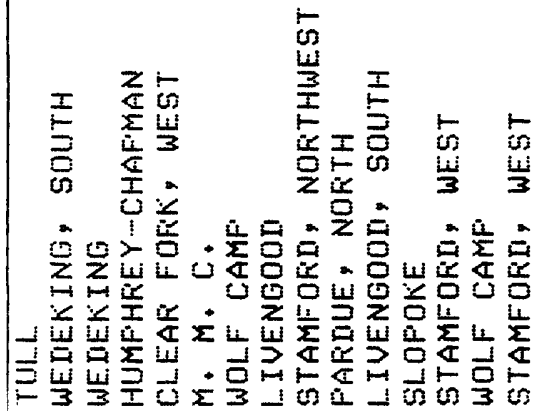


NANANANAN

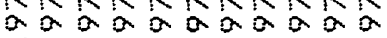

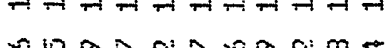
míño $0 \% \pi+2000$ on $\infty+\cdots=200$ Non

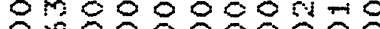

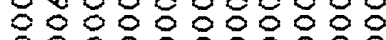

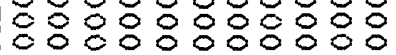

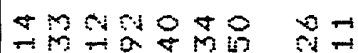

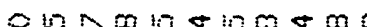

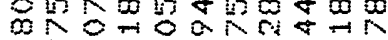
$m \infty$ is 20, $\rightarrow m m<\infty a n a n m$ is is th th is is 0000 20202002020

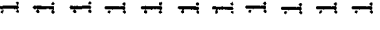

峾焉

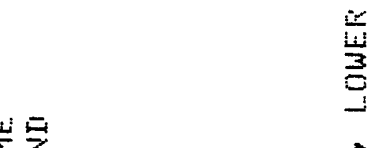

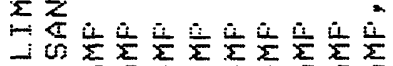

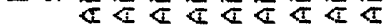

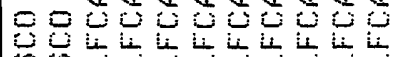

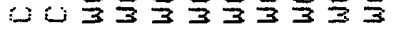
की

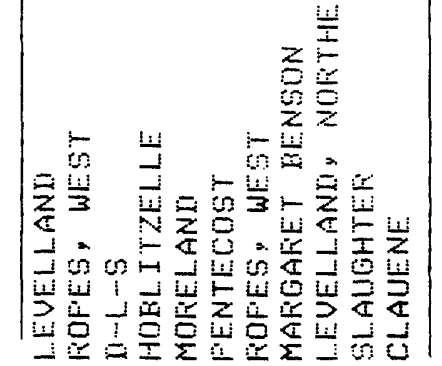

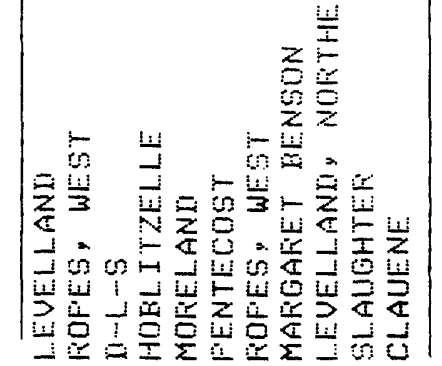

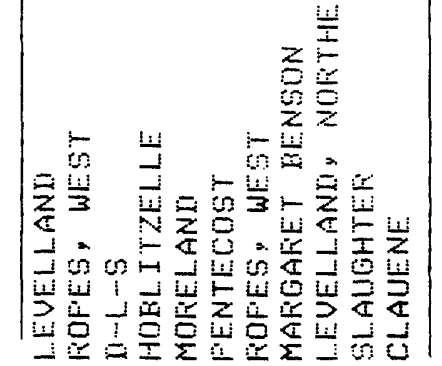

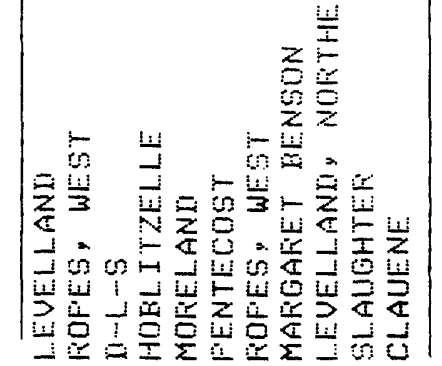

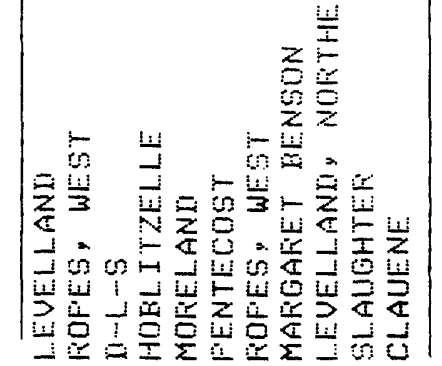

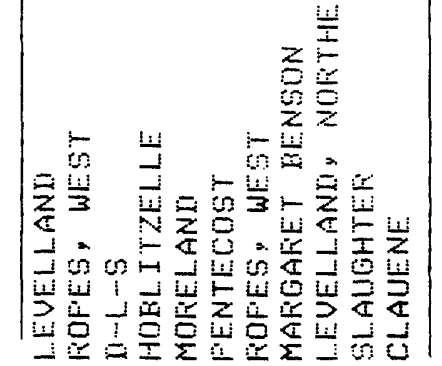

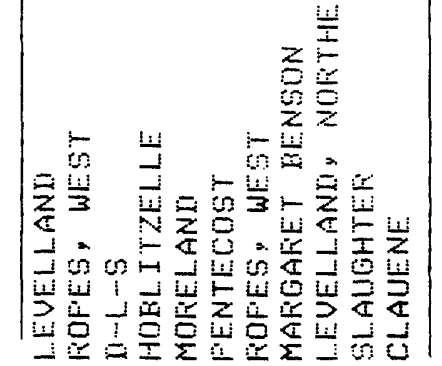

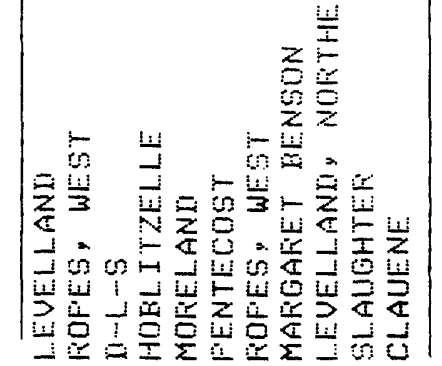

ANANANANANANANRN

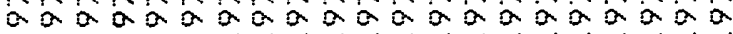

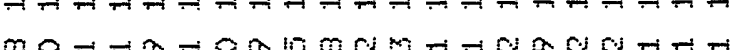

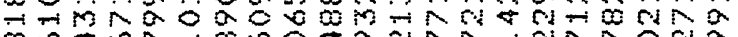
m 1

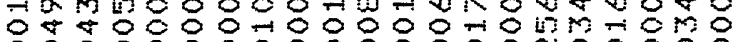

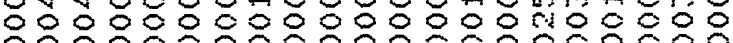

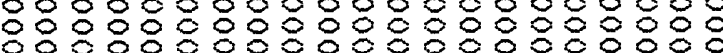

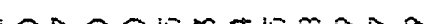

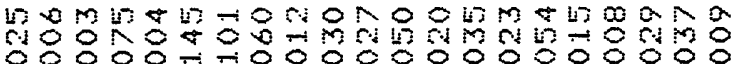
maooonmonrinomiomonmon

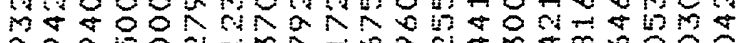

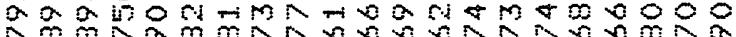

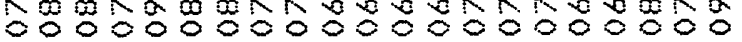
on manoon

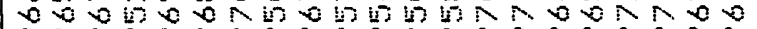

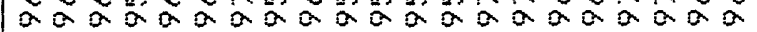

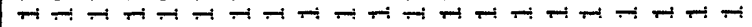

$\operatorname{tin}$ $a_{\rightarrow \rightarrow \infty} a_{i}$ is $m 0$ is $\infty 02$ 象 응 88 10808 $\infty$ 050 0000 $\infty \infty 20$ $20 \infty \infty$ tis in $\operatorname{ton} 0$ oon 15 $a_{-2}=2$

L 단 난 $3 \frac{1}{5}$

$\frac{4}{1 ! i t}$

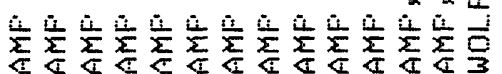

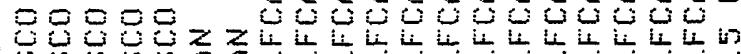

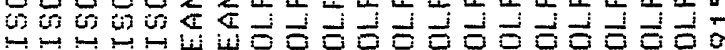

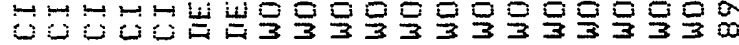

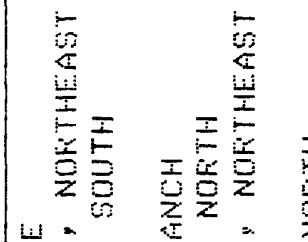

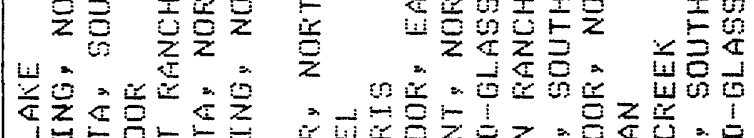

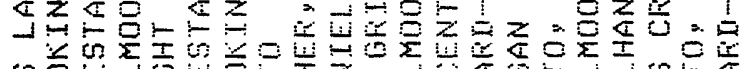

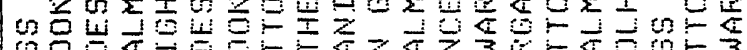

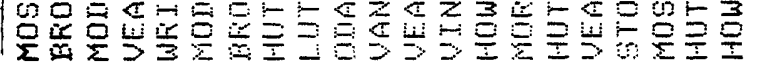


NRNANAN

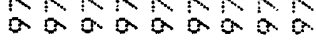
$\rightarrow \rightarrow \rightarrow+\pi \rightarrow+\rightarrow$

$+-7-82 \infty \infty$ iक सेख्रा 100002

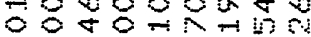

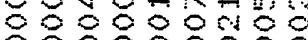

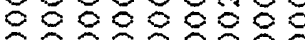

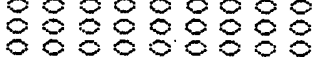
mol m व०6

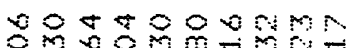
का

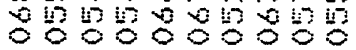

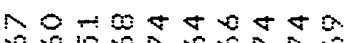
2002000200 $\approx \rightarrow+\infty=\alpha+\infty$

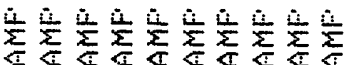

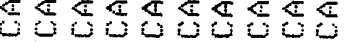

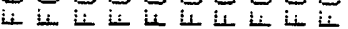

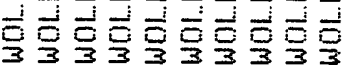

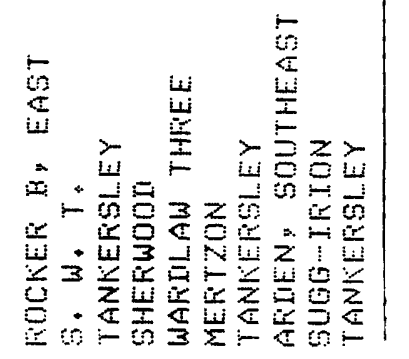

NNANAN 000000000

a -

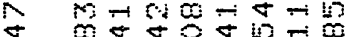

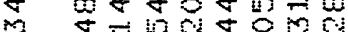
O NM\&NM母

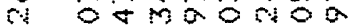

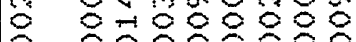

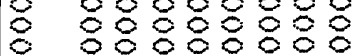

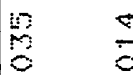
8 - 000 ano

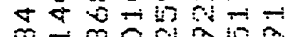

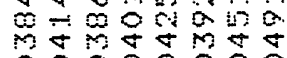
¿ ammmarmo is $00000 N N N$ in 2020020000

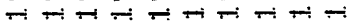

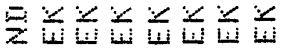

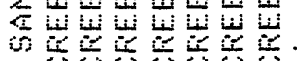

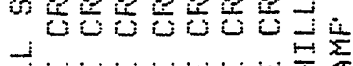

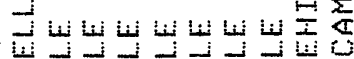

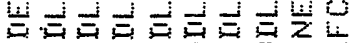

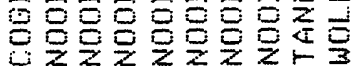

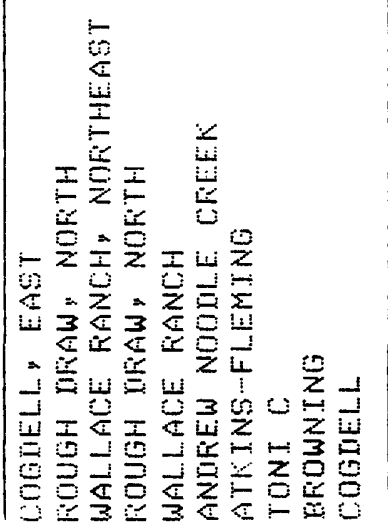

ANANANANANAN $\alpha 2 \alpha 2 \alpha 2 \alpha 2020202$

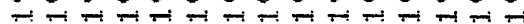
$\infty$ in का के ontonomoob00\%

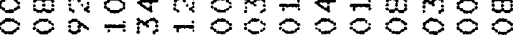

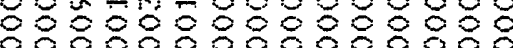

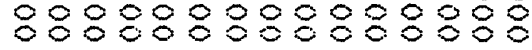

8

68ㅇㅇㅇ ino0000000mbn am

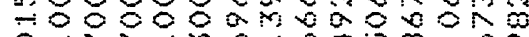
न 4 min \%०0006000000000

0 -immmm

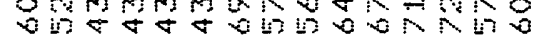
a 2002002002020

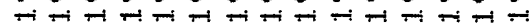

望

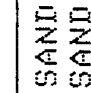

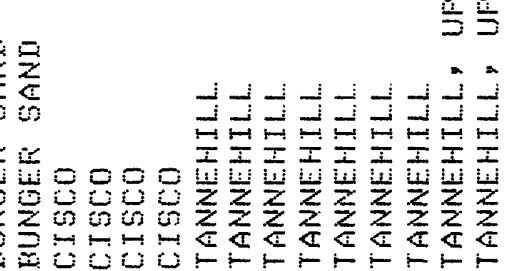

$\sum_{i}^{T}$

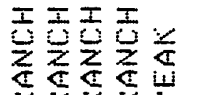

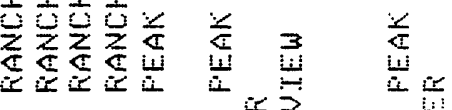

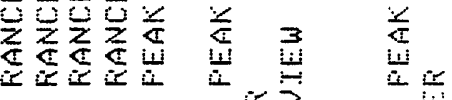

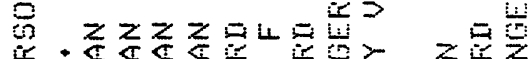

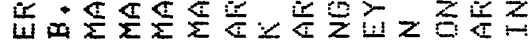

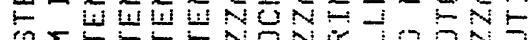

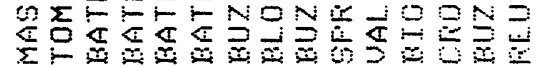

NAR 2020 $80 m$ कón $0-m$ id 8280 8888 4580 is $\rightarrow 0$ $000 a$ in 2 in $a 020$

$$
\rightarrow-\rightarrow+\longrightarrow
$$
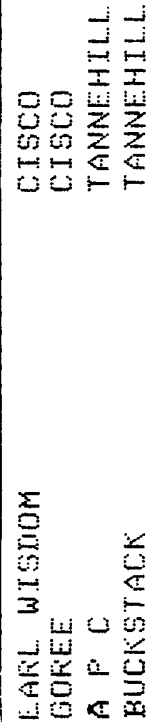
NANANANANANANANANANANANANANANANANANANANANANANAN

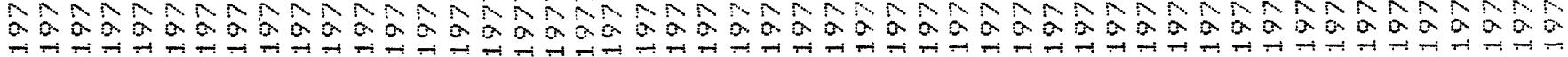

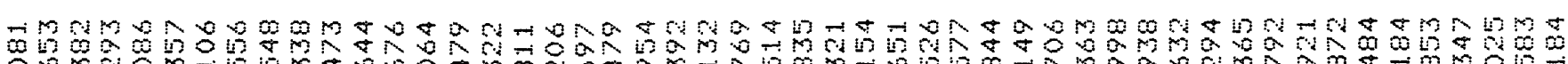

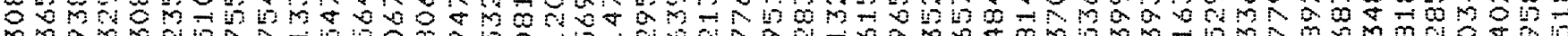
mmam

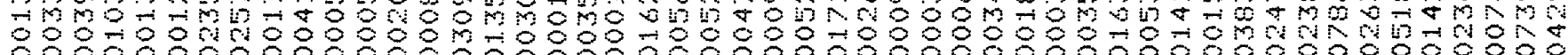

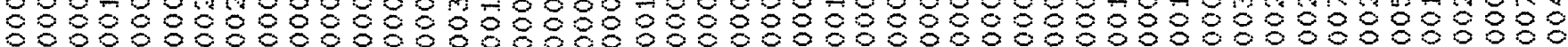

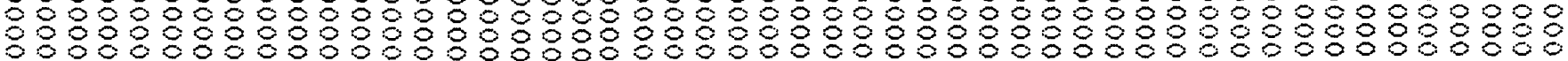

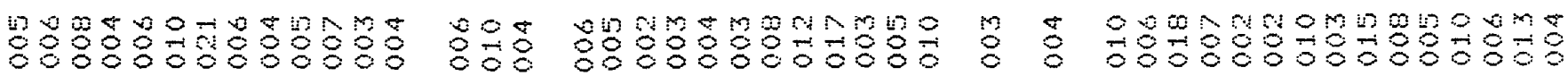

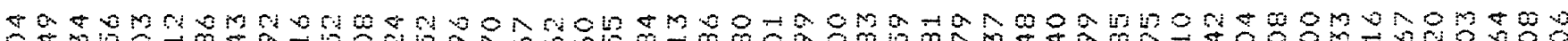
otmis -

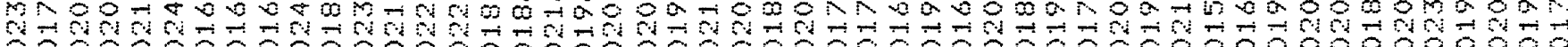
o000000000000000000000000000000000000000000000000

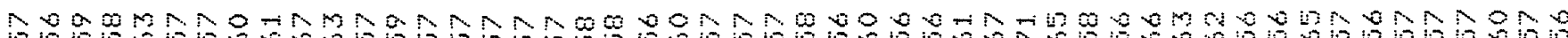

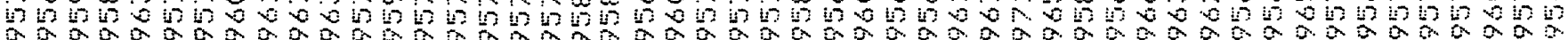

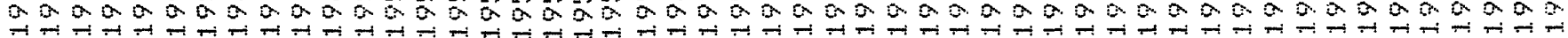

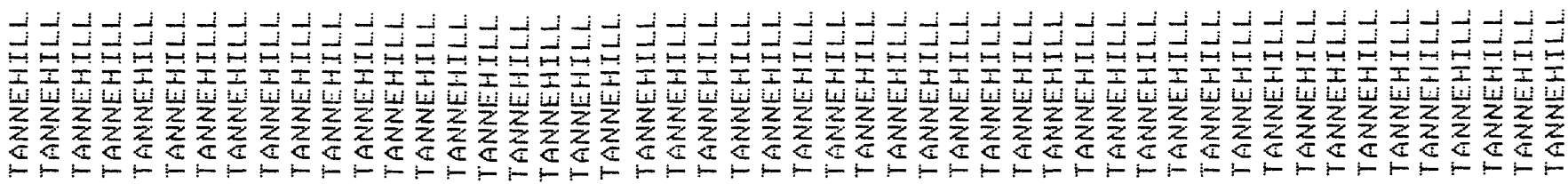

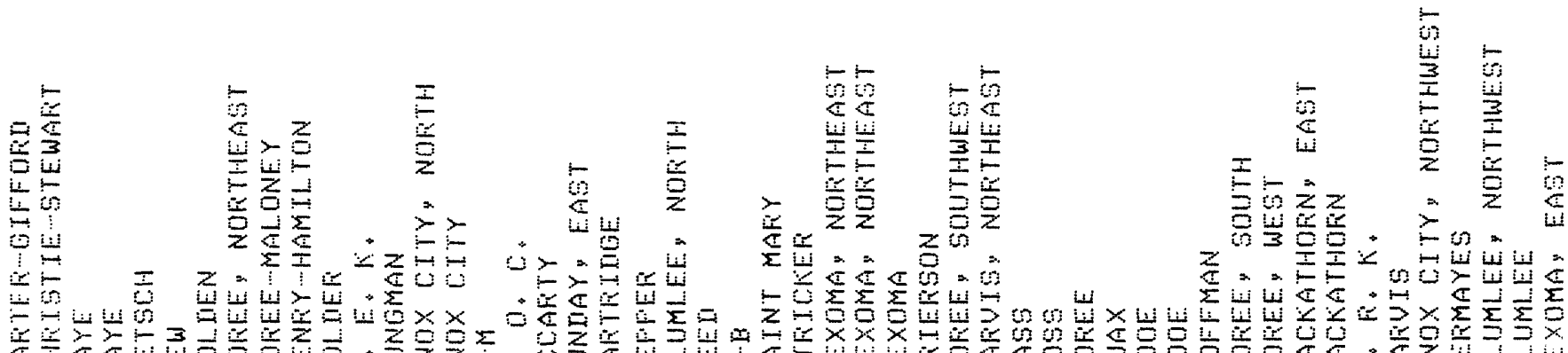

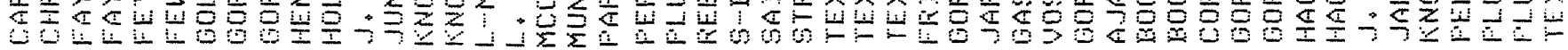


NANANANAN

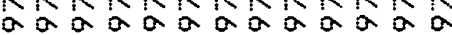

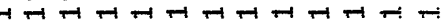
in 000 ก

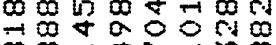
का 00 स

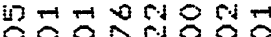

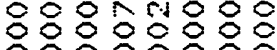
$8.8: 8: 8:$

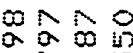
$2 \pi+0$ in $\mathrm{a}^{\circ} \mathrm{o}$ 78 응 $\circ 080$ ㅇㅇㅇㅇㅇㅇㅇㅇㅇ 융융 oomanomammon m

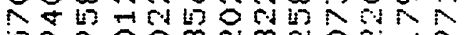

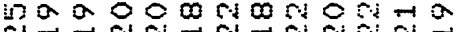

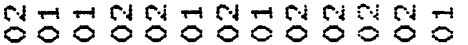
mmanton

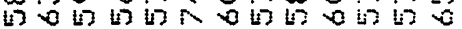

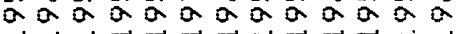
HनHनHनH
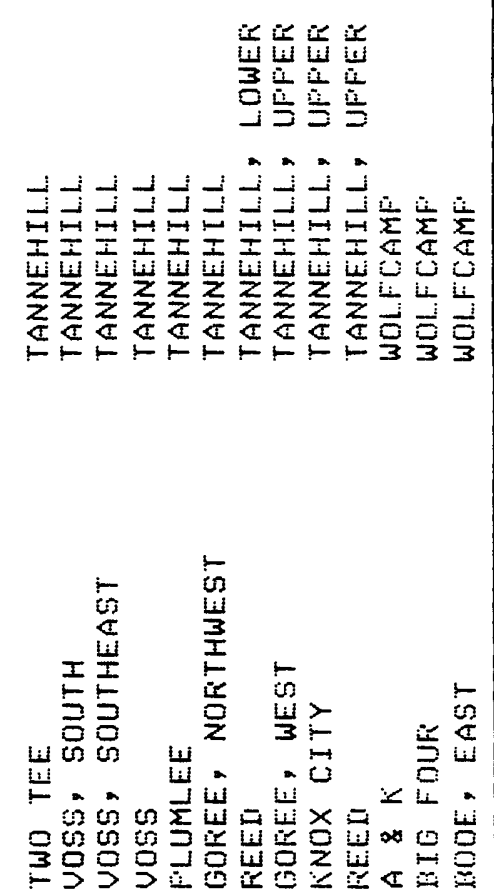

NNDNENANENANANANE 20202020202020202020

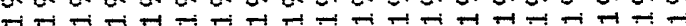

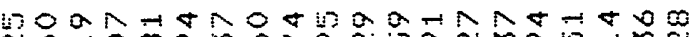
की $00 \%$ t

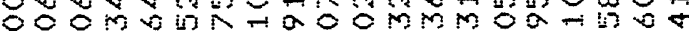

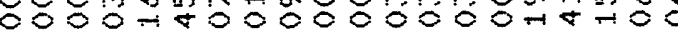
60080

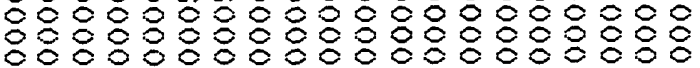

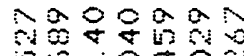

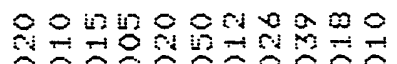
arthn

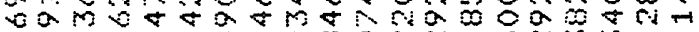
in

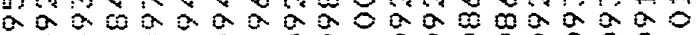

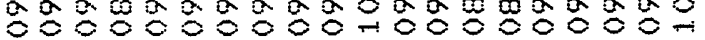

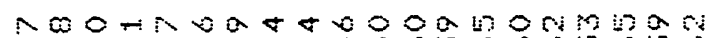

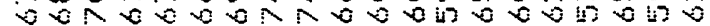
20200200200200020202

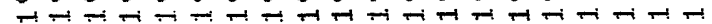

点 $\frac{\pi}{2}$

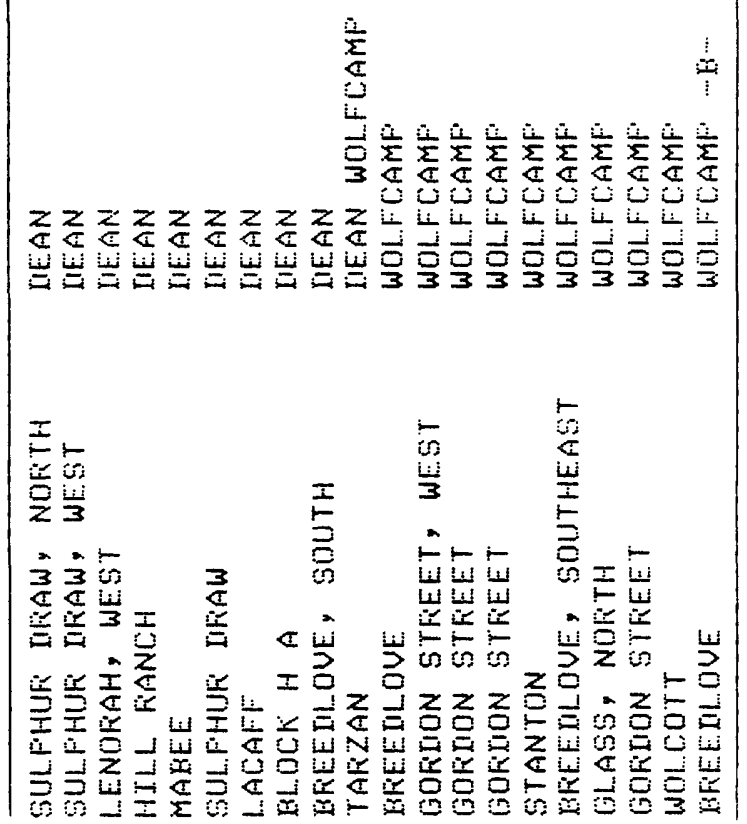

ANANANAN a2a 2020200

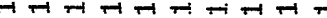

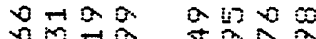
ती nim mob

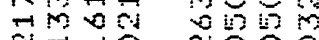
तु. 응ㅇㅇㅇ

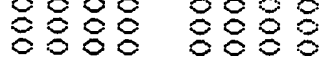

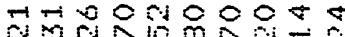

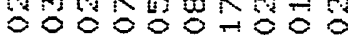
+roo요의 trioconmtm ii 00 in $00 \pi \infty \%$

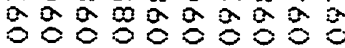
ond an $000+$ in 1 iो 000 करतaरaखa 20

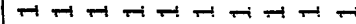

$\frac{i}{\frac{5}{\alpha}} \frac{i}{\tau}$

要思

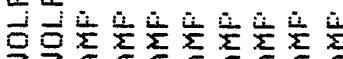

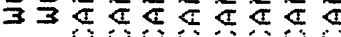

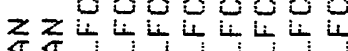

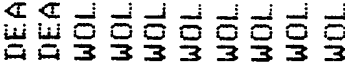

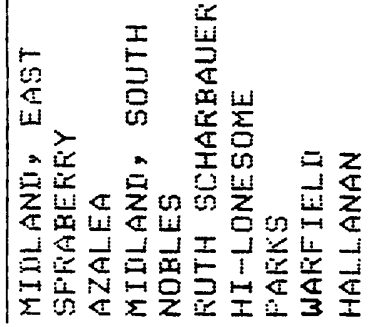


NNN 2202 matio $20 \mathrm{sin}$ $8 m a$ लिख

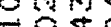

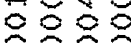
280

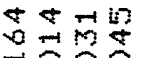
a $\operatorname{tin} 5$ $4 a a m$ mo 680 $0 \infty \infty$ is $20 a 2$ - بt $\rightarrow+1$

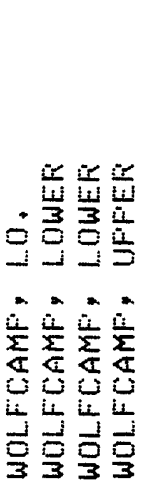
ㅇㅇㅇㅇㅇㅇ

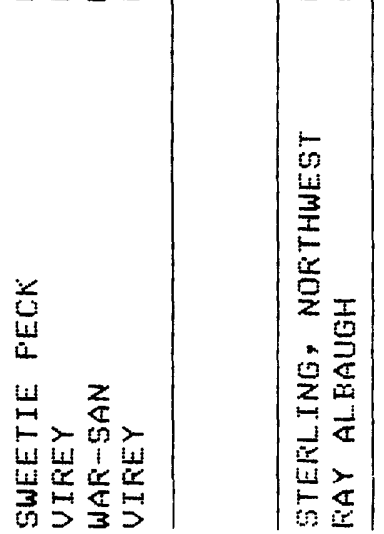

NANAN 2202220

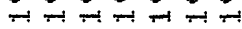
तोo\& $\infty-10 \log 2$ if $00 \%+0$ \& $40+60$ 응

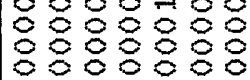

NANANANANANANANAN

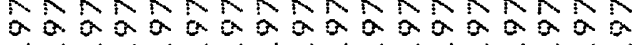

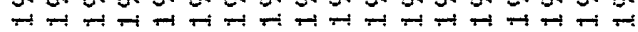

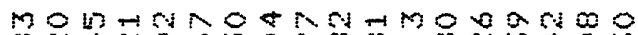

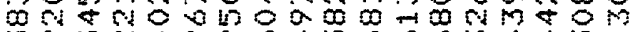

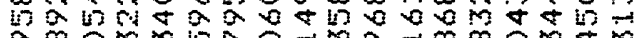

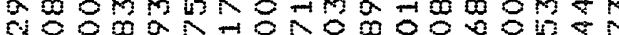

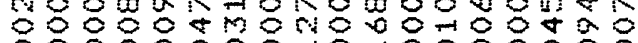

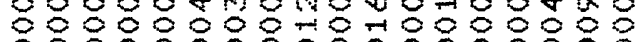

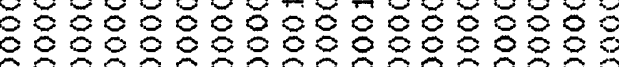

un ofo

웅용

स6808

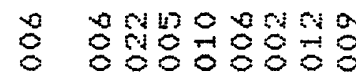

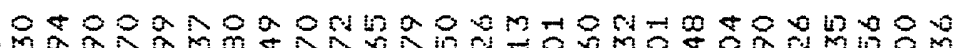
a

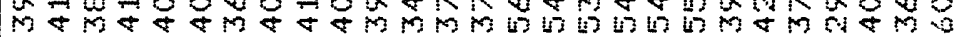
000000000000000000000000000

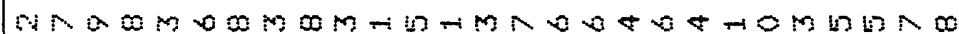

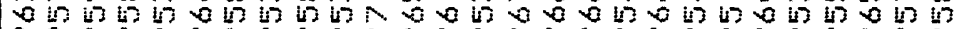

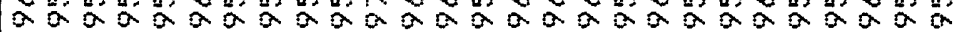

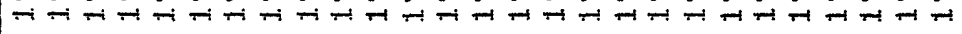

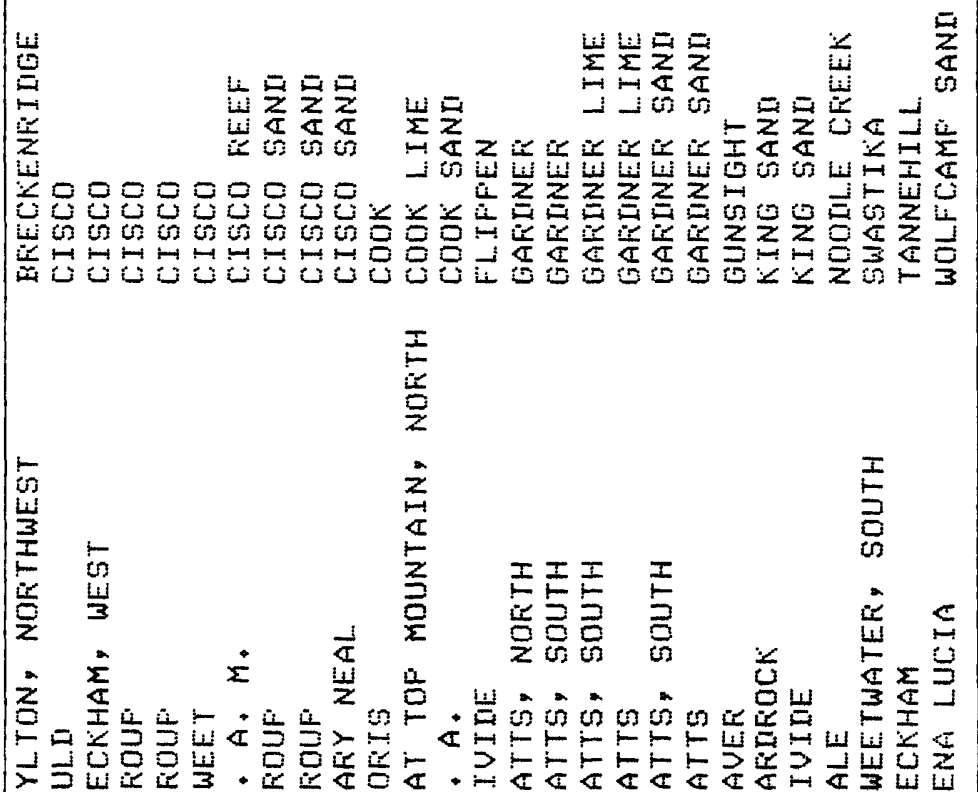

$\infty$

$\rightarrow m$

$i \infty$

in

88

$\hat{2}$

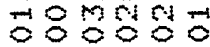

om arimo 0 \& 0000

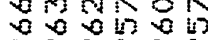
a a a a

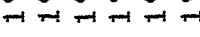

989989 句的要品 000000

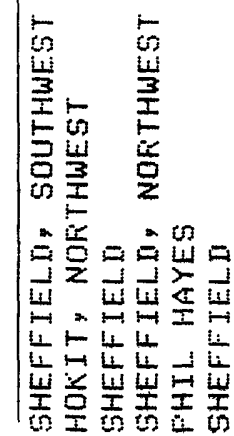




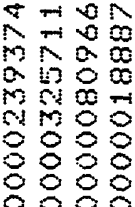

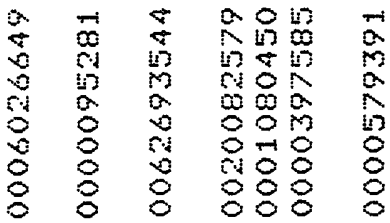

$\begin{array}{ll}8 & 12 \\ 0 & 0 \\ 0 & 0 \\ 0 & 0 \\ 0 & 0 \\ 0 & 0 \\ 0 & 0\end{array}$

$2 \mathrm{in}$

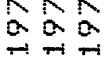

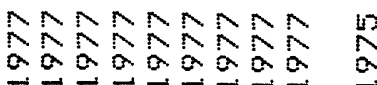

$\overbrace{2}^{12}$

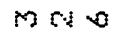

$\rightarrow \infty$

का

\&

08

888

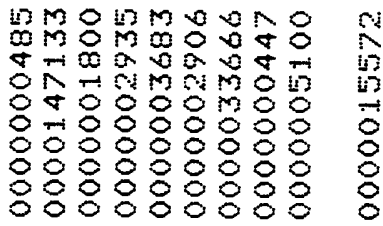

ลÑNمN

âa $2 \hat{a}$

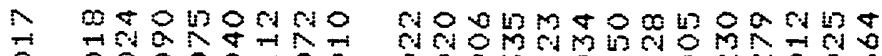

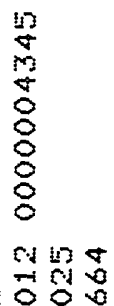

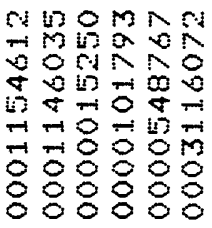

सम

ति

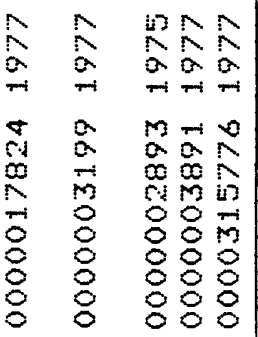

$+\infty \infty \infty m$

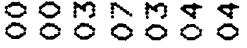

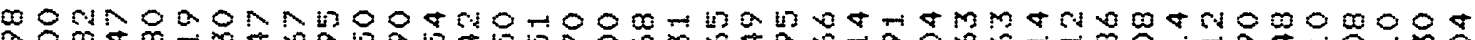

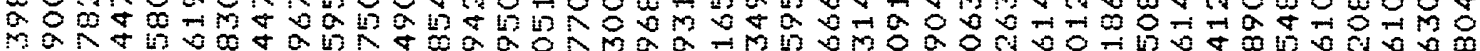

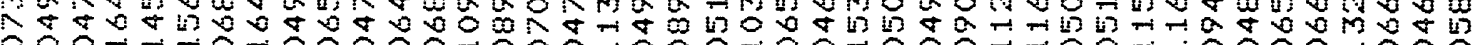

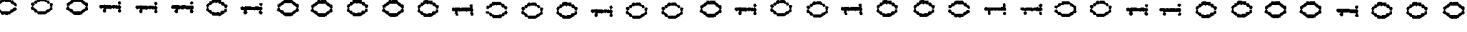

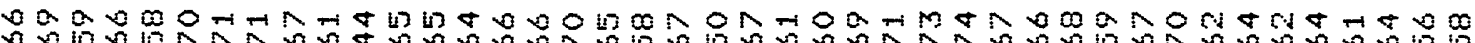

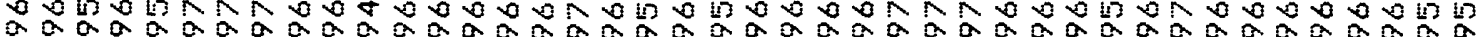

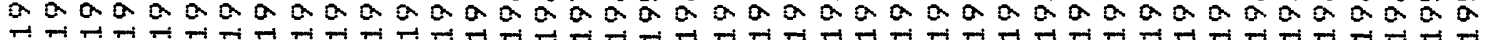

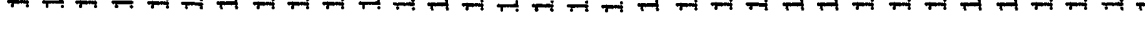

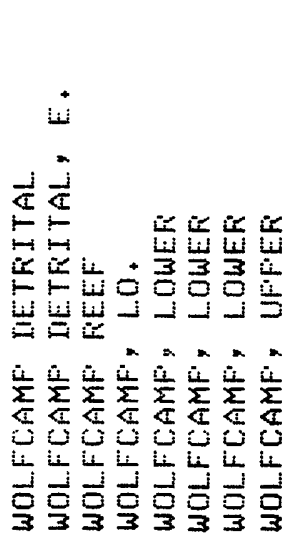

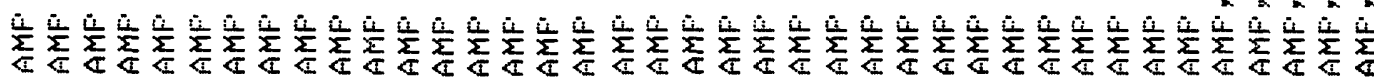

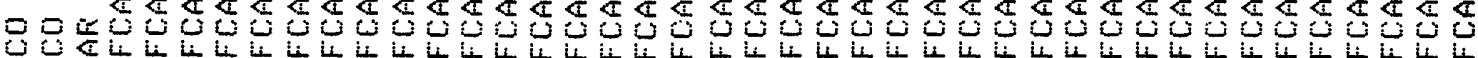

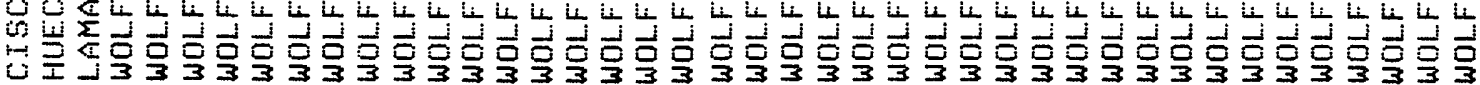

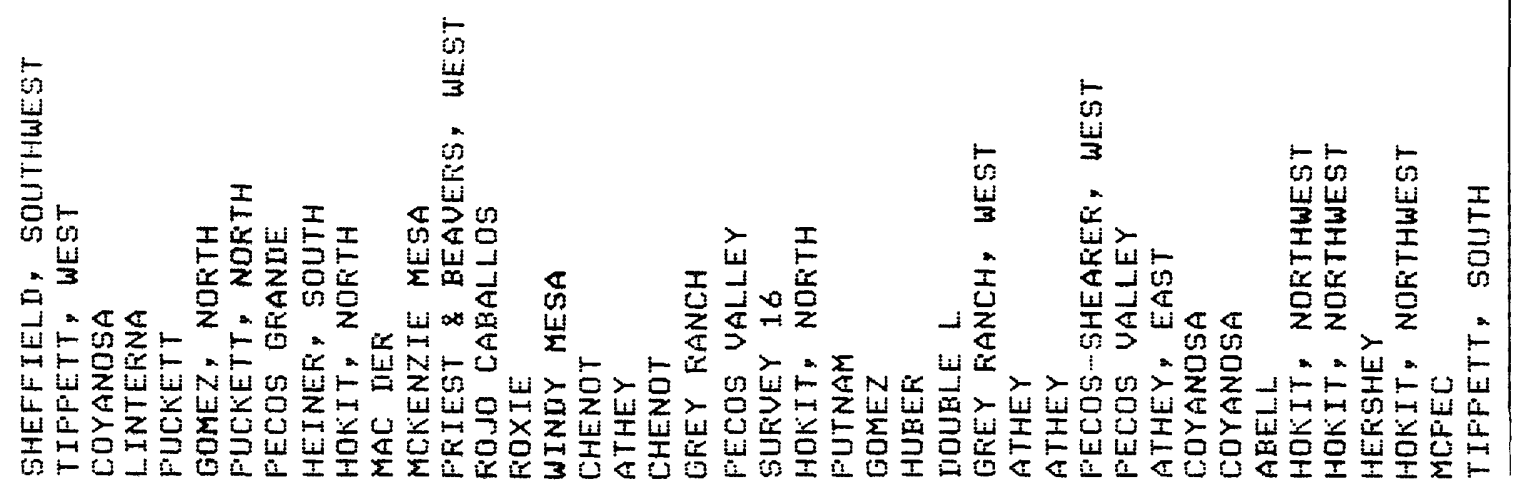

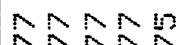
âa 20 Timiti $m \infty \ln M$ $\sin 0$

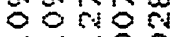

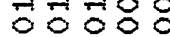

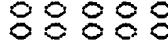
88888 anoma कमील

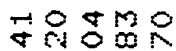

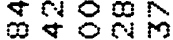
R 00000 mano 100002 a20.20 (1)

$z_{a} z_{i} z_{i} z_{i} \underset{c}{z}$

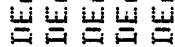

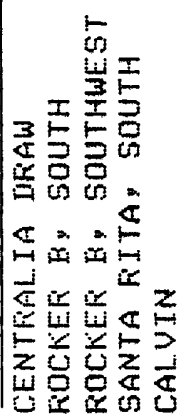


NRNA $2 \Omega 2 N \Omega$ $\rightarrow \rightarrow \rightarrow+4 \rightarrow$

OMMNOS on wam

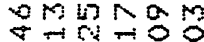
$00088 \div$

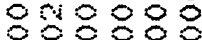

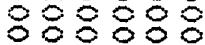
0 ता सम तथ $+00000$ 의

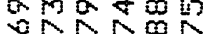
000000

012000 is 0 a 2012 $\rightarrow \rightarrow \rightarrow+\rightarrow+1$
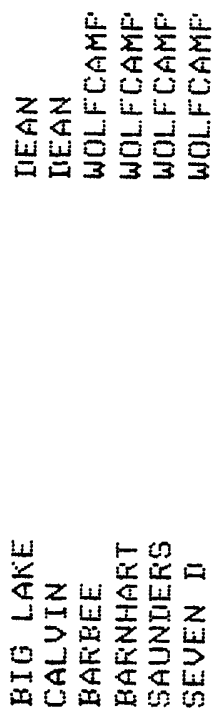

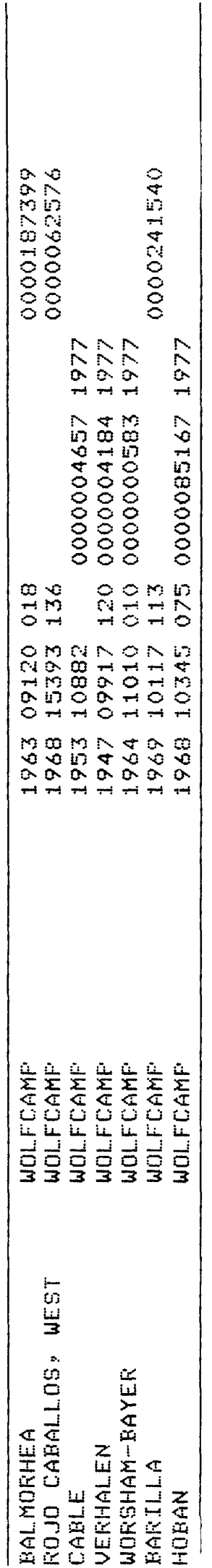

NNANNNNNA 20202020200

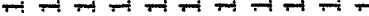
5 ला RONRTR nNAm in

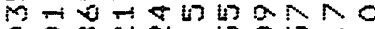
으메

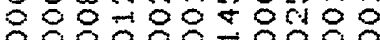
O००0\%\% 88888888080

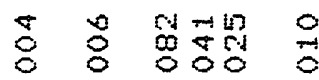
म०० 808

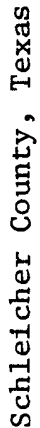

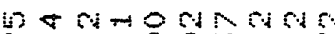

2

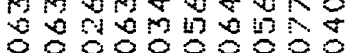
20 in a a a a a a a a

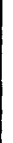

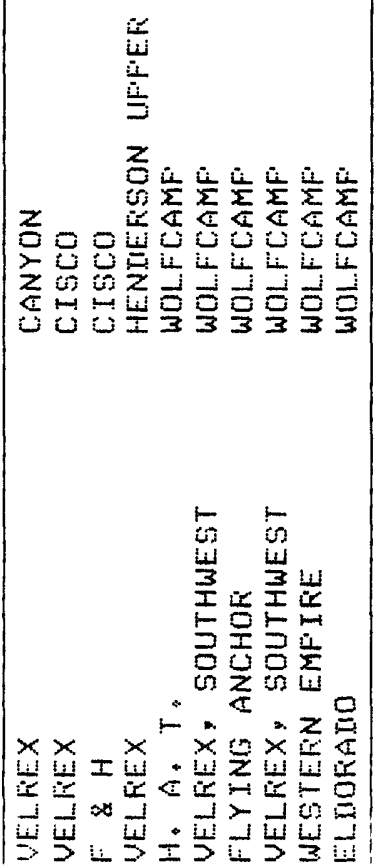

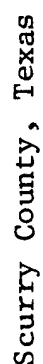

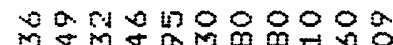

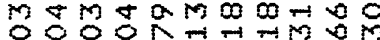

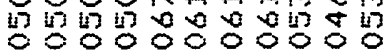
DON

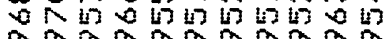
$a_{i \rightarrow \rightarrow \rightarrow \rightarrow \rightarrow a}$

百

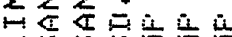
的的文文空 0900000000

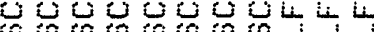

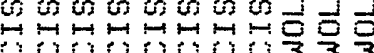

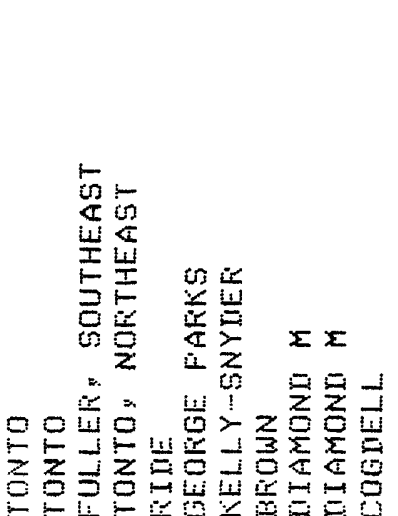


0
0
8
8
0
8
8
8
8

FNANANAN

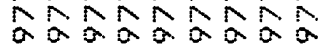

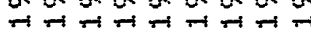

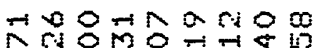

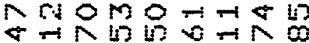

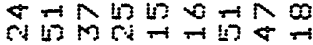

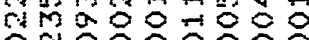

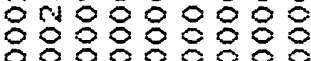

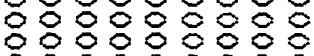

NANAN $\sin \sin$ $a_{\rightarrow+1000}^{2}$

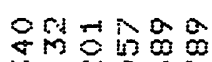
marion iूa $\rightarrow$ ra $\rightarrow$ in 68888

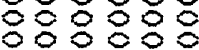

$\operatorname{Nan}$ NNNAN $a 0 a 00$ nN 8 m IN सक 2000 -imbon 0 mo

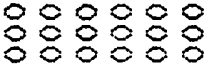

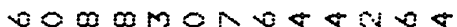

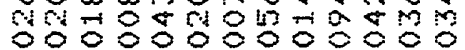

tra

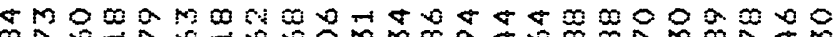
a arif OOOOSO000000000000000000

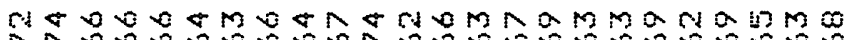
सर०000000010000000000000

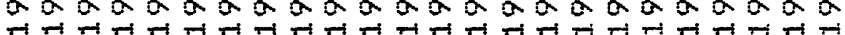
年

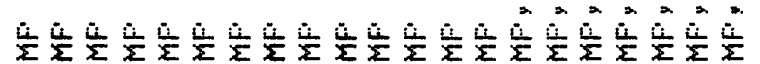

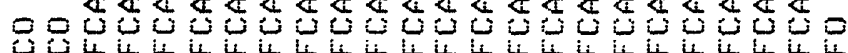

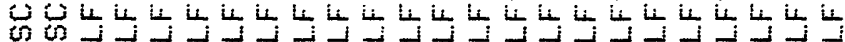

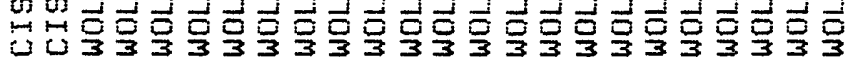

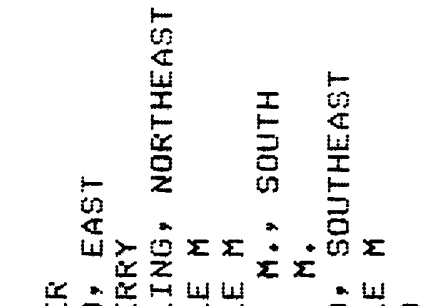

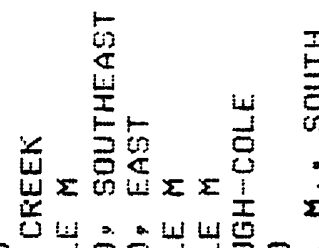
志增

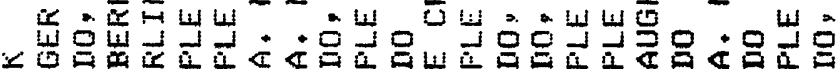

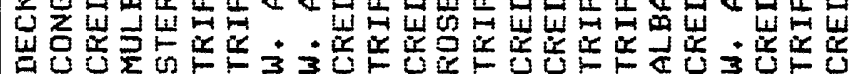

NANANANANANANANA सीNAOANANANANA $\rightarrow$ at

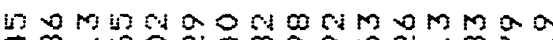
म it

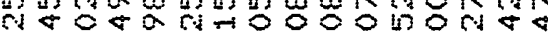

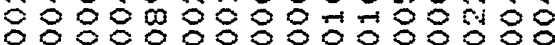
ก.

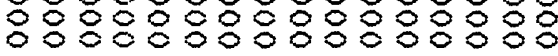

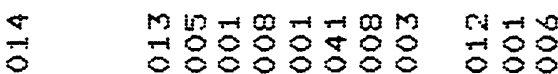

- 4 nmm NMmin mo

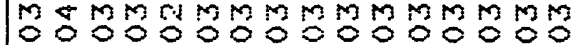

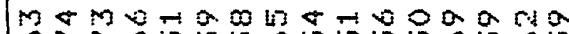

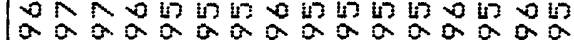

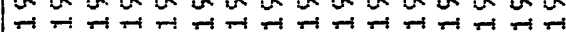

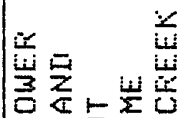

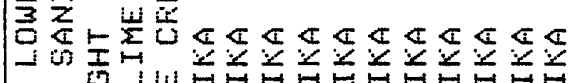

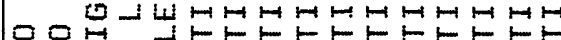

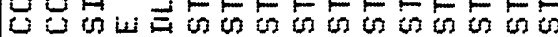
क ज 2

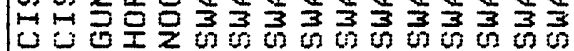

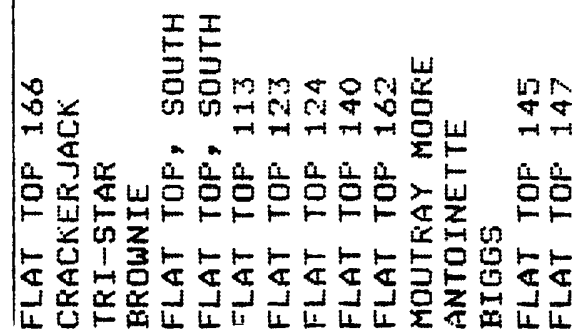


NRNRNRNRNANRNRNAR o.

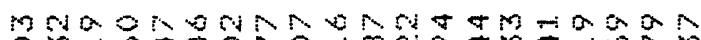
OM-G

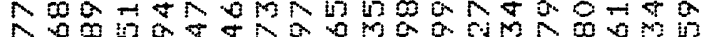

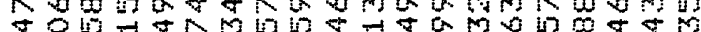

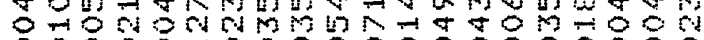
0080000000900008080

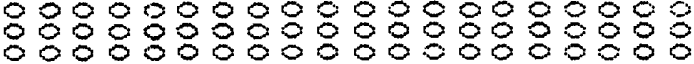

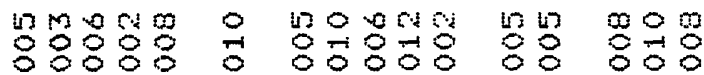

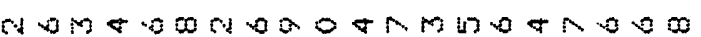

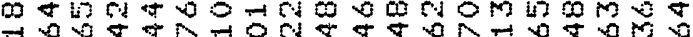
मी m5000000000000000000

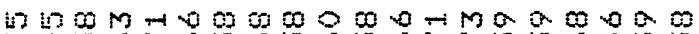
15.

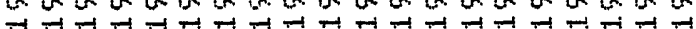
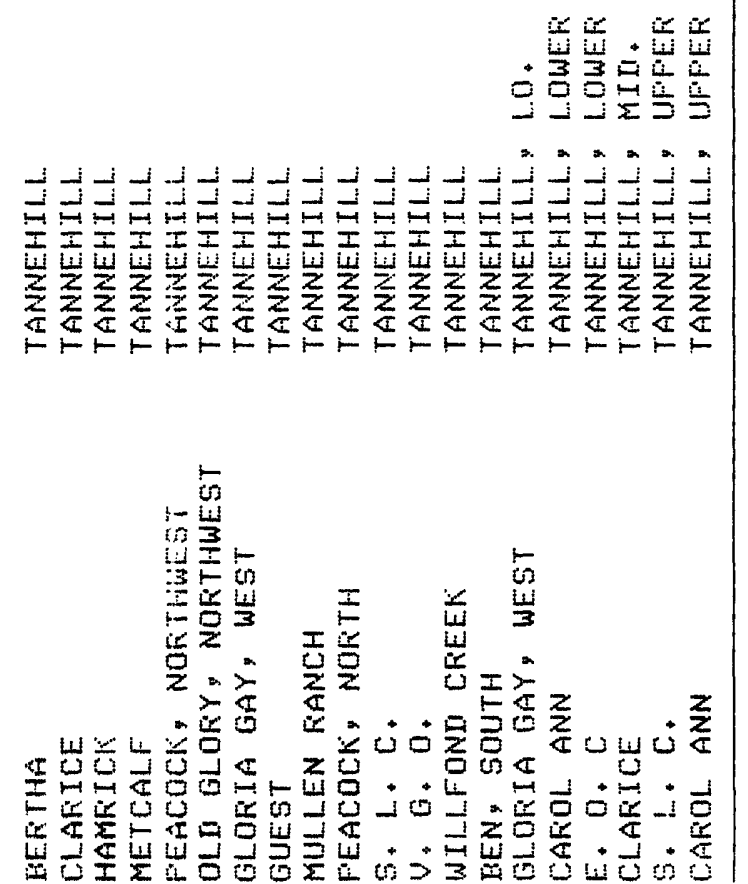

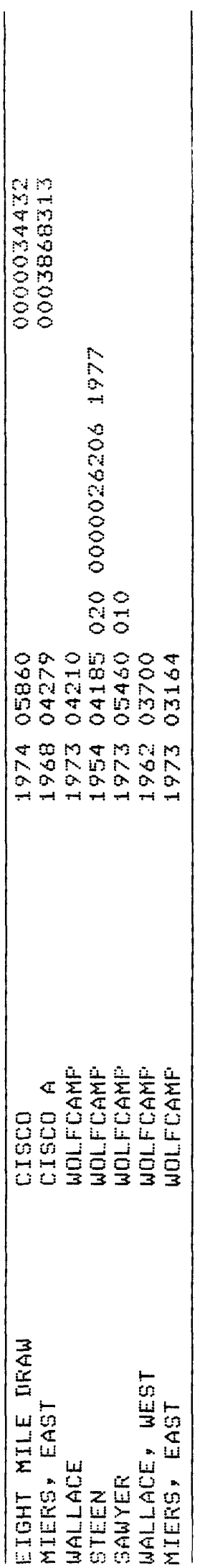

30
84
89
88

1
8
8
8
8

$\operatorname{lig}_{0}$

8 두요

30

0050

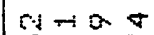

$008 \pi$

离

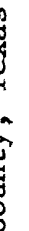

NNNNN 202020 monoma in 700000

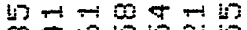
0 \& 0 in औल

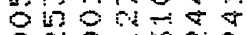
ㅇㅇㅇㅇㅛ

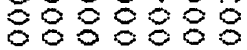
명여요 on का मा

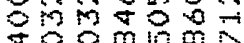
₹8801002 कारषण स 10 in 6 to 40 a

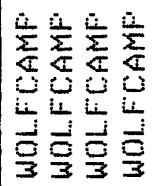

4

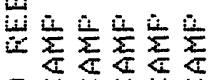
000000 604

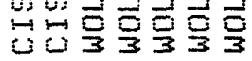

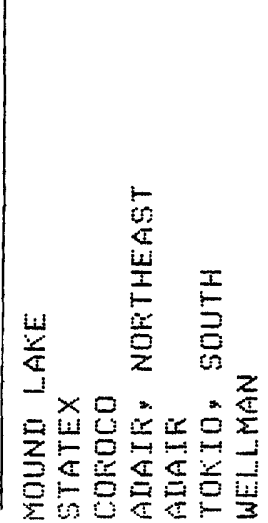




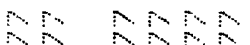

$\infty 200$

$\infty-\infty \cdots 0$

16 bo

$6 \%$ की

08 स

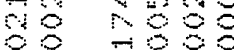

6ㅇㅇㅇㅇㅇㅇ

$8 \% 8380$

औ

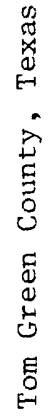

0 insom

0 मा की

$m+\varangle 4 \& 0$

000000

1000

50200

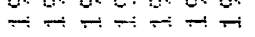

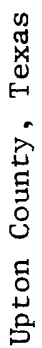

$\Xi$

27

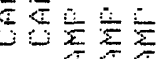

800000

$8090=$

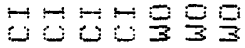

$\stackrel{4}{=}$

要

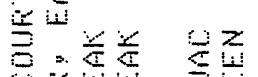

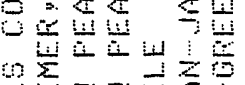

$9 \sum_{1} z z=2$

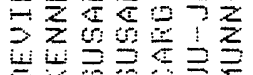

8

8

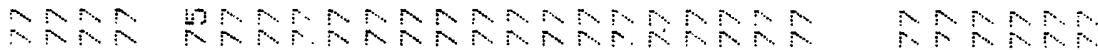

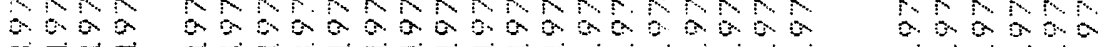

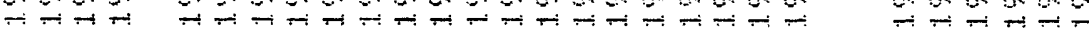

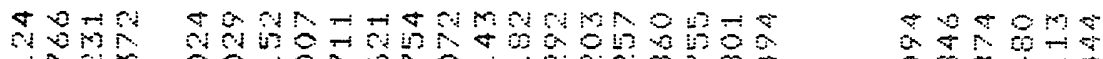
तीक की की

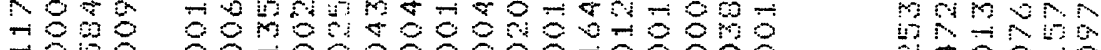

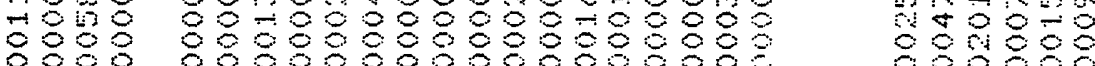

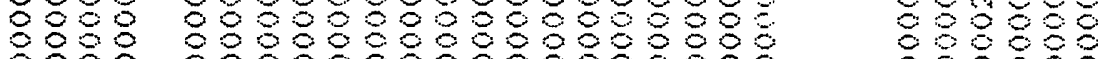

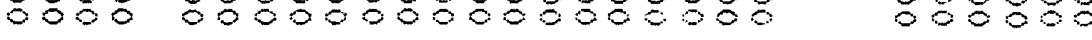

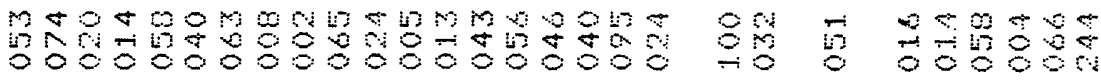

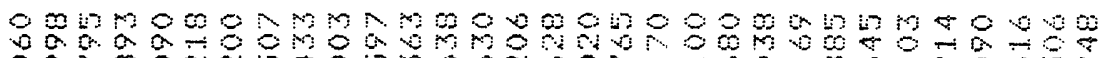

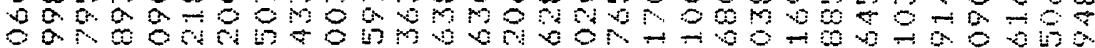

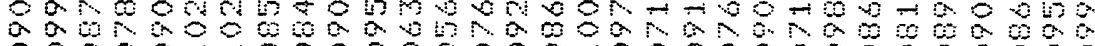
$00000-7000000000+60000006006000$ ob Hoth 안

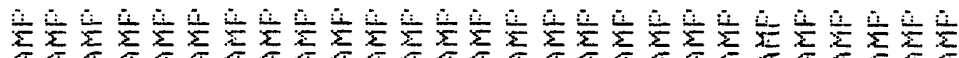

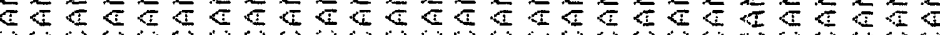

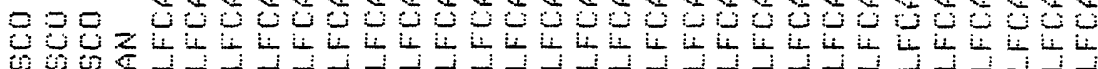

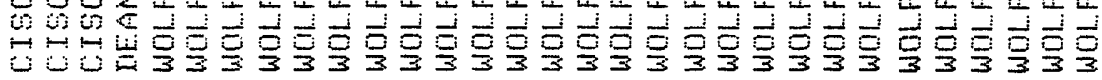

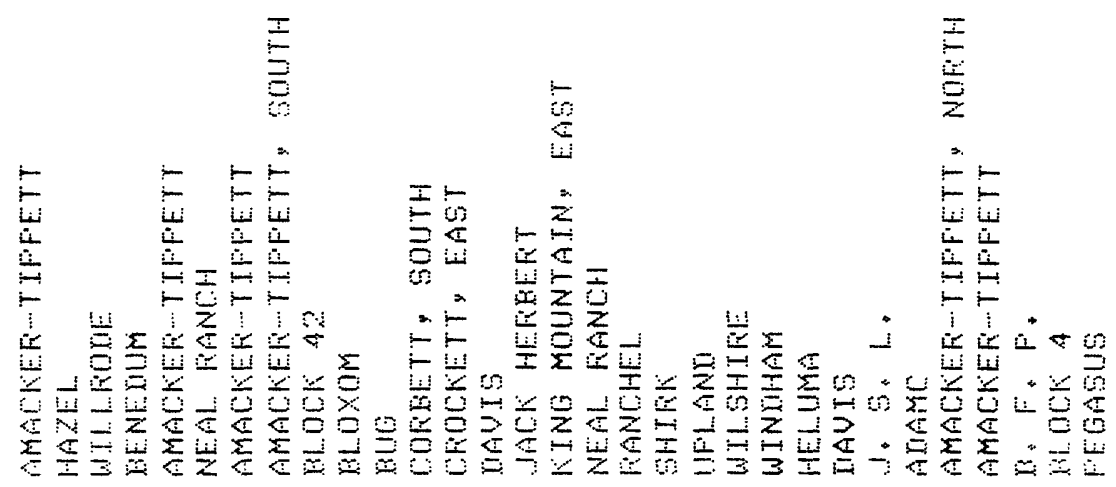




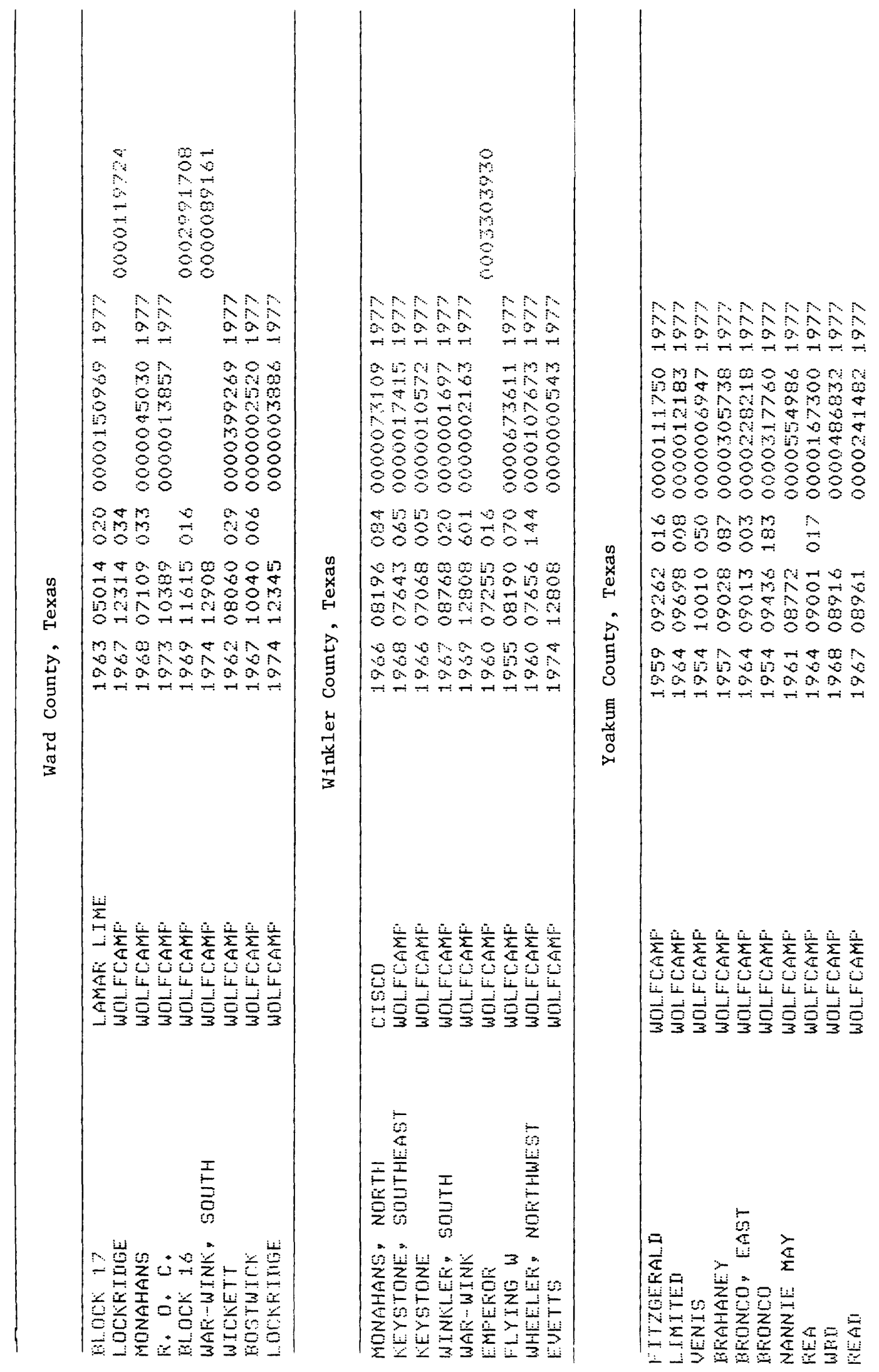


Selected Bibliography

Adams, J. E., Frenzel, H. N., Rhodes, M. L., and Johnson, D. P., 1951, Starved Pennsylvanian Midland Basin (Texas): American Association of Petroleum Geologists Bulletin, v. 35, p. 2600-2606.

Armstrong, A. K., 1962, Stratigraphy and paleontology of Mississippian system in southwestern New Mexico and adjacent southeastern Arizona: New Mexico Bureau of Mines and Mineral Resources, Memoir 8. 95 p. 1967, Biostratigraphy and carbonate facies of the Mississippian Arroyo Penasio Formation, north-central New Mexico: New Mexico Bureau of Mines and Mineral Resources, Memoir 20. 80 p.

Backman, G. O., 1975, New Mexico: in MeKee, E. D., and Crosby, E. J., editors, Paleotectonic Investigations of the Pennsylvanian System in the United States: USGS Professional Paper 853, part 1, p. 233-243.

Ball, S. M., Pollard, W. D., and Roberts, J. W., 1977, Importance of phylloid algae in development of depositional topography: in Frost, S. H., Weiss, M. P., and Saunders, J. B., editors, Reefs and Related Carbonates - Ecology and Sedimentology: American Association of Petroleum Geologists, Studies in Geology No. 4, p. 239-260.

Bowsher, A. L., 1948, Mississippian bioherms in the northern part of the Sacramento Mountains, New Mexico: The Compass, v. 25, p. 21-28.

Butler, J.H., 1977, Geology of the Sacramento Mountains, Otero County, New Mexico: West Texas Geological Society Publication No. 1977-68. 216 p.

Choquette, P. W., and Traut, J. D., 1963, Pennsylvanian carbonate reservoirs, Ismay Field, Utah and Colorado: in Bass, R. 0., and Sharps, S. L., editors, Shelf Carbonates, Paradox Basin: Four Corners Geological Society, Fourth Field Conference. $273 \mathrm{p}$.

Cline, L. M., 1959, Preliminary studies of the cyclical sedimentation and paleontology of upper Virgil strata of the La Luz area: in Guidebook, Sacramento Mountains, 
New Mexico: Permian Basin Section of the Society of Economic Paleontologists and Mineralogists and Roswell Geological Society. 306 p.

Cys, J. M., and Mazzullo, S. J., 1977, Biohermal submarine cements, Laborcita Formation (Permian), northern Sacramento Mountains, New Mexico: in Butler, J., editor, Geology of the Sacramento Mountains, Otero County, New Mexico: West Texas Geological Society Publication No. 1977-68, p. 39-51. Delgado, D. J., and Pray, L. C., 1977, Stop "C-3" The Laborcita Formation: in Butler, J. H., editor, Geology of the Sacramento Mountains, Otero County, New Mexico: West Texas Geological Society Publication No. 1977-68, p. 173-183. Elias, G. K., 1963, Habitat of Pennsylvanian algal bioherms, Four Corners area: in Bass, R. O., and Sharps, S. L., editors, Shelf Carbonates, Paradox Basin: Four Corners Geological Society, Fourth Field Conference, p. 185-203. Irwin, Jr., C. D., 1963, Producing carbonate reservoirs in the Four Corners area: in Bass, R. 0., editor, Shelf Carbonates of the Paradox Basin: Four Corners Geological Society, Fourth Field Conference Guidebook, p. 144-148.

Jerome, S. E., Campbe11, D. D., Wright, J. S., and Vitz, H. E., 1965, Geology and ore deposits of the Sacramento (High Rolls) mining district, Otero County, New Mexico: New Mexico Bureau of Mines and Mineral Resources Bulletin 86.30 p. Kerr, Jr., S. D., 1969, Algal-bearing carbonate reservoirs of Pennsylvanian age, West Texas and New Mexico: (Abstract), American Association of Petroleum Geologists Bulletin, v. 53, p. 726-727.

Konishi, K., and Wray, J. L., 1961, Eugonophyl1um, a new Pennsylvanian and Permian algal genus: Journal of Paleontology, v. 35, p. 659-666.

Kottlowski, F. E., 1960a, Summary of Pennsylvanian sections in southwestern New Mexico and eastern Arizona: New Mexico Bureau of Mines and Mineral Resources Bulletin 66. $187 \mathrm{p}$. 1968, Sedimentational influence of Pedernal Uplift: (Abstract), American Association of Petroleum Geologists Bulletin, v. 52, no. 1, p. 197. 
Laudon, L. R., and Bowsher, A. L., 1941, Mississippian formations of the Sacramento Mountains, New Mexico: American Association of Petroleum Geologists Bulletin, v. 25 , p. $2107-2160$.

1949, Mississippian formations of southwestern New Mexico: Geological Society of America Bulletin, v. 60 , p. 1-88.

LeMay, W. J., 1972, Empire Abo Field, southeast New Mexico: in King, R. E., editor, Stratigraphic Oil and Gas Fields: American Association of Petroleum Geologists Memoir 16, p. 472-480.

Lohmann, K. C., and Meyers, W. J., 1977, Microdolomite inclusions in cloudy prismatic calcites: a proposed criterion for former high-magnesium calcites: Journal of Sedimentary Petrology, v. 47, p. 1078-1088.

Macintyre, I. G., 1977, Distribution of submarine cements in a modern Caribbean fringing reef, Galeta Point, Panama: Journal of Sedimentary Petrology, v. 47, p. 503-516.

Malek-Aslani, M., 1970, Lower Wolfcampian Reef in Kemnitz Field, Lea County, New Mexico: American Association of Petroleum Geologists Bulletin, v. 54, p.2317-35. Mazzullo, S. J., and Cys, J. M., 1979, Marine aragonite sea-floor growths and cements in Permian phylloid algal mounds, Sacramento Mountains, New Mexico: Journal of Sedimentary Petrology, v. 49, p. 917-936.

McKee, E. D., and Crosby, E. J., 1975, Paleotectonic Investigations of the Pennsylvanian system of the United States: USGS Professional Paper 853, part III. Meyer, R. F., 1966, Geology of Pennsylvanian and Wolfcampian rocks in southeast New Mexico: New Mexico Bureau of Mines and Mineral Resources, Memoir 17. $123 \mathrm{p}$.

Meyers, W. J., 1974, Carbonate cement stratigraphy of the Lake Valley Formation (Mississippian), Sacramento Mountains, New Mexico: Journal of Sedimentary Petrology, v. 44, p. 837-861.

1978, Carbonate cements: their regional distribution and interpretation in Mississippian limestones of southwestern New Mexico: Sedimentology, v. 25, p. $371-400$. 
, and James, A. T., 1978, Stable isotopes of cherts and carbonate cements of the Lake Valley Formation (Mississippian), Sacramento Mountains, New Mexico: Sedimentology, v. 25, p. 105-124.

, and Lohmann, K. C., 1978, Microdolomite-rich syntaxial cements: proposed meteoric-marine mixing zone phreatic cements from Mississippian limestones, New Mexico: Journal of Sedimentary Petrology, v. 48, p. 475-488. Miller, F., 1969, The San Andres reef zone: in Summers, W. K., and Kottlowski, F. E., editors, The San Andres Limestone, a Reservoir for Oil and Water in New Mexico: New Mexico Geological Survey Special Publication No. 3, p. $27-31$. Neumann, A. C., Kofoed, J. W., and Keller, G. H., 1977, Lithoherms in the Straits of Florida: Geology, v. 5, p. 4-10.

Otte, Jr., C., 1954, Wolfcampian reefs of the northern Sacramento Mountains, Otero County, New Mexico: (Abstract), Geological Society of America Bulletin, v. 65, p. 1291-1292.

1959a, The Laborcita Formation of late Virginian-early Wolfcampian age of the northern Sacramento Mountains, Otero County, New Mexico: in Guidebook, Sacramento Mountains, New Mexico: Permian Basin Section of the Society of Economic Paleontologists and Mineralogists and Roswell Geological Society. $306 \mathrm{p}$.

1959b, Late Pennsylvanian and early Permian stratigraphy of the northern Sacramento Mountains, Otero County, New Mexico: New Mexico Bureau of Mines and Mineral Resources Bulletin 50. $111 \mathrm{p}$. , and Parks, Jr., J. M., 1963, Fabric studies of Virgil and Wolfcamp bioherms, New Mexico: Journal of Geology, v. 73, p. 380-396. Oppe1, T. W., 1959, The Pennsylvanian-Permian contact in lower Fresnal Canyon, Sacramento Mountains, New Mexico: in Guidebook, Sacramento Mountains, New Mexico: Permian Basin Section of the Society of Economic Paleontologists and Mineralogists and Roswe1l Geological Society. 306 p. 
Parks, Jr., J. M., 1958, Plate-shaped calcareous algae in late Paleozoic rocks of mid-continent: (Abstract), Geological Society of America Bulletin, v. 69, p. 1627.

1962, Reef-building biota from late Pennsylvanian reefs, Sacramento Mountains, New Mexico: (Abstract), American Association of Petroleum Geologists Bulletin 46, p. 274 .

1975, Diagenetic obliteration of frame-building organisms in undolomitized late Paleozoic reefs: (Abstract), American Association of Petroleum Geologists, Annual Meeting Abstracts, v. 2, p. 58-59.

1977a, Origin of early vuggy porosity in carbonate mudbank buildups, Pennsylvanian and Permian, Sacramento Mountains, New Mexico: American Association of Petroleum Geologists Bulletin, v. 61, p. 819-820.

1977b, Paleoecological evidence on the origin of the Dry Canyon Pennsylvanian bioherms: in Geology of the Sacramento Mountains, Otero County, New Mexico, West Texas Geological Society Publication 1977-68, p. 27-32.

Peterson, J. A., and Ohlen, H. R., 1963, Pennsylvanian shelf carbonates, Paradox Basin: in Bass, R. O., editor, Shelf Carbonates of the Paradox Basin, Four Corners Geological Society, Fourth Field Conference Guidebook, p. 65-79. Plumley, W. J., and Graves, R. W., 1953, Virgilian reefs of the Sacramento Mountains, New Mexico: Journal of Geology, v. 61, p. 1-16.

Pray, L. C., 1949, Pre-Abo deformation in the Sacramento Mountains, Otero County, New Mexico: (Abstract), Geological Society of America Bulletin, v. 61, p. 1914-1915. 1952, Stratigraphy of the escarpment of the Sacramento Mountains, Otero County, New Mexico: Ph.D. Dissertation, California Institute of Technology, Pasadena, California. 370 p. 1953, Upper Ordovician and Silurian stratigraphy of Sacramento Mountains, Otero County, New Mexico: American Association of Petroleum Geologists 
Bulletin, v. 37, p. 1894-1918.

1954, Outline of the stratigraphy and structure of the Sacramento Mountain

Escarpment: New Mexico Geological Society Fifth Field Conference Guidebook, Southeastern New Mexico, p. 92-107.

1958a, Fenestrate bryozoan core facies, Mississippian bioherms, southwestern

United States: Journal of Sedimentary Petrology, v. 28, p. 261-273.

1958b, Pennsylvanian sedimentation of the Sacramento Mountains area, New

Mexico: (Abstract), Third Annual Meeting Permian Basin Section of Society of Economic Paleontologists and Mineralogists, Midland, Texas. p. 7. 1959, Outline of the stratigraphy and structure of the Sacramento Mountain escarpment of New Mexico: in Guidebook, Sacramento Mountains, New Mexico: Permian Basin Section of Society of Economic Paleontologists and Mineralogists and Roswel1 Geological Society, p. 86-130.

1961, Geology of the Sacramento Mountains escarpment, Otero County, New

Mexico: New Mexico Bureau of Mines and Mineral Resources Bulletin 35.144 p. 1965a, Limestone clastic dikes in Mississippian bioherms, New Mexico: (Abstract), Geological Society of America Special Paper 82, p. 154-155. 1965b, Clastic limestone dikes and marine cementation, Mississippian bioherms, New Mexico: (Abstract), Permian Basin Section of Society of Economic Paleontologists and Mineralogists, Third Seminar on Sedimentation, Program, p. 21-22. 1969, Micrite and carbonate cement, genetic factors in Mississippian bioherms: (Abstract), North American Paleontologic Conference, Program. 1975, Mississippian shelf-edge and basin facies carbonates, Sacramento Mountains and southern New Mexico region: Dallas Geological Society Guidebook. $140 \mathrm{p}$. , and Bowsher, A. L., 1952, Fusselman Limestone of the Sacramento Mountains, New Mexico: (Abstract), Geological Society of America Bulletin, v. 63, p. 1342. , and Graves, R. L., 1954, Desmoinesian facies of the Sacramento Mountains, 
New Mexico: (Abstract), Geological Society of America Bulletin, v. 65, p. 1295. , and Wray, J. L., 1963, Porous algal facies (Pennsylvanian) Honaker Trail,

San Juan Canyon, Utah: en Bass, R. O., and Sharps, S. L., editors, Shelf

Carbonates, Paradox Basin: Four Corners Geological Society Fourth Field Conference. $273 \mathrm{p}$.

Thornton, D. E., and Gaston, Jr., H. H., 1968, Geology and development of the Lusk Strawn Field, Eddy and Lea Counties, New Mexico: American Association of Petroleum Geologists Bulletin, v. 52, p. 66-81.

Toomey, D. F., 1976, Paleosynecology of a Permian plant dominated marine community: Neues Jahrb. Geologie u. Paläontologie Abh., v. 152, p. 1-18. , and Winland, H. D., 1973, Rock and biotic facies associated with Middle Pennsylvanian (Desmoinesian) algal buildup, Nena Lucia Field, Noland County, Texas: American Association of Petroleum Geologists Bulletin, v. 57, p. 10531074 .

, Wilson, J. L., and Rezak, R., 1977a, Growth history of a late Pennsylvanian phylloid algal organic buildup, northern Sacramento Mountains, New Mexico: in Geology of the Sacramento Mountains, Otero County, New Mexico: West Texas Geological Society Publication No. 1977-68. p. 9-26. 1977b, Evolution of Yucca Mound Complex, late Pennsylvanian phylloid algal buildup, Sacramento Mountains, New Mexico: American Association of Petroleum Geologists Bulletin, v. 61, p. 2115-2133.

Vest, E. L., 1970, Oil fields of Pennsylvanian-Permian Horseshoe Atoll, West Texas: in Halbouty, M., editor, Geology of Giant Petroleum Fields: American Association of Petroleum Geology Memoir 14, p. 185-203.

Wilson, J. L., 1967, Cyclic and reciprocal sedimentation in Virgilian strata of southern New Mexico: Geological Society of America Bulletin, v. 78, p. 805818. 1969, Influence of local structure in sedimentary cycles of Beeman and Holder 
Formations, Sacramento Mountains, Otero County, New Mexico: in Cyclic Sedimentation in the Permian Basin (2nd edition), West Texas Geological Society Pub1ication No. 1969-56, p. 100-114.

1975a, Carbonate Facies in Geologic History, Springer-Verlag, New York. 469 p. 1975b, Regional Mississippian facies and thickness in southern New Mexico and Chihuahua: in Pray, L. C., editor, Mississippian Shelf-Edge and Basin Facies Carbonates, Sacramento Mountains and southern New Mexico Region: Dallas Geologica1 Society Guidebook, p. 124-128.

1977, Regional distribution of phylloid algal mounds in Late Pennsylvanian and Wolfcampian strata of southern New Mexico: in Butler, J. H., editor, Geology of the Sacramento Mountains, Otero County, New Mexico, West Texas Geologica1 Society Publication No. 1977-68, p. 1-7.

Wray, J. L., 1959, Origin of some Pennsylvanian algal bioherms in southwestern United States: in Guidebook, Sacramento Mountains, New Mexico: Permian Basin Section of Society of Economic Paleontologists and Mineralogists and Roswe11 Geologica1 Society, p. 38.

1963, Pennsylvanian algal banks, Sacramento Mountains, New Mexico: in Geoeconomics of the Pennsylvanian Marine Banks of Southeast Kansas: Kansas Geological Society, 27th Annual Field Conference, p. 129-133.

1968, Late Paleozoic phylloid algal limestone in the United States: Proceedings, 23rd International Geologic Conference, Prague, v. 8, p. 113-119. 1975, The puzzling Paleozoic phylloid algae: a Holocene answer in Squamariacean calcareous red algae: (Abstract), American Association of Petroleum Geologists and Society of Economic Paleontologists and Mineralogists, Annual Meeting Abstracts, v. 2, p. 82-83. 1977, Calcareous A1gae, E1sevier, New York, p. 52-54. 